\title{
Representation Learning for Information Extraction
}

Ehsan Amjadian

A thesis submitted to the Faculty of Graduate and Postdoctoral Affairs in partial fulfillment of the requirements for the degree of

Doctor of Philosophy

in Cognitive Science

Carleton University

Ottawa, Ontario

(C) 2019

Ehsan Amjadian 


\begin{abstract}
Distributed representations, predominantly acquired via neural networks, have been applied to natural language processing tasks including speech recognition and machine translation with a success comparable to sophisticated state-of-the-art algorithms. The present thesis offers an investigation of the application of such representations to information extraction. Specifically, I explore the suitability of applying shallow distributed representations to the automatic terminology extraction task, as well as the bridging reference resolution task. I created a dataset as a gold standard for automatic term extraction in the mathematical education domain. I carefully assessed the performance of the existing terminology extraction methods on this dataset. Then, I introduce a novel method for automatic terminology extraction for one word terms, and I evaluate the performance of the novel algorithm in various terminological domains. The introduced algorithm leverages the distributed representation of words from the local and global perspectives to encode syntactic, semantic, association, and frequency information at the same time. Furthermore, this novel algorithm can be trained with a minimal number of data points. I show that the algorithm is robust to the change of domain, and that information can be transferred from one technical domain to another, leveraging what we call anchor words with consistent semantics shared between the domains. As for the bridging reference resolution task, a dataset is built on the letter portion of the Open American National Corpus and I compare the performance of a preliminary method against a majority class baseline.
\end{abstract}




\section{Acknowledgements}

The great sacrifices and contributions made by others enriched my journey over the past years. Words cannot do justice in capturing their help and sacrifices, however, this is an attempt to acknowledge them.

I would like to thank my brother Amir Amjadian and my mother Dr. Khadijeh Eshaghi for their support and sacrifice that first and foremost made this endeavor possible. I could travel, as a result, to the other side of the world in pursuit of knowledge and to contribute to the scientific community. Amir and Khadijeh, without your great help and sacrifice this journey would have never started. Thank you!

I would like to thank my dear wife, Mahsa Raeisi Ardali, for her unconditional support during the writing stage of this dissertation. Her devotion and encouragement strengthened my steps forward.

Immeasurable gratitude gos to my supervisors: Professor Raj Singh and Professor Diana Inkpen. Dr. Singh took me under his wing and made Ottawa feel like home. My eyes were opened and my mind freed by our discussions, his clarity in thoughts, reasoning and writing, his vast experience in research, his absolute command of formal and computational semantics and pragmatics, and above all, his invaluable mentorship and friendship. Dr. Inkpen noticed my passion for natural language processing, machine learning, and deep learning, and nurtured them with her depth and breadth of knowledge in the field of artificial intelligence and computer science, turning thoughts into ideas, and ideas into experiments. I could not have asked for a better supervision and mentorship.

I would like to thank my thesis committee members, Professor Xiaodan Zhu and Professor Robert West, for the many inspiring discussions and conversations in NLP, 
information extraction, word embeddings, and high dimensional data structures as well as their great feedback for the present thesis.

Great thanks go to Professor Patrick Drouin for his pioneering work in automatic terminology extraction as well as his invaluable comments on the thesis. I was fortunate to be one of the countless individuals who have been inspired by his work.

Many thanks go to Professor Christopher Cox for his invaluable feedback on the thesis that resulted from a close assessment of the ideas and experiments in the document which lead to their further refinement.

I could not have wished for better lab mates and friends than Roxana Barbu and Prasadith Kirinde Gamaarachchige. Prasadith brought an ocean of cutting-edge skills in software engineering and web development to the lab, in addition to his radiating serenity. Roxana made many extended hours of research seem normal and pleasant by her diligence and team work, even though we worked on different projects. Thank you both for being such great friends and all the thought-provoking conversations.

Great thanks go to my friend and colleague Professor Muhammad Rizwan Abid. We had many inspiring conversations from the very beginning of my journey, many of which resulted in great academic work. Roxana and Muhammad both kindly proofread the present document and made many great suggestions that lead to its improvement.

A significant portion of the work in automatic terminology extraction comes from the years of collaboration with Professor T.Sima Paribakht and Professor Farahnaz Faez as well as their contributions before I joined the project. Working with them was an absolute honor and pleasure. I benefited much from their advices and insights.

Great thanks go to Liane Dubreuil for the warm welcome to the department and 
for removing any administrative obstacle from my path.

The present work benefited much from great efforts by Jeol Baylis and Christopher Genovesi constructing the bridging reference corpus.

I would like to thank Amir Gharavi for all the detailed mathematical discussions as well as being a great friend over the past years. 


\section{Contents}

Abstract ........................... ii

Acknowledgements ............................ iii

1 Introduction 1

1.1 The Tasks to Tackle . . . . . . . . . . . . . . . . 1

1.2 Automatic Terminology Extraction (ATE) . . . . . . . . . . 3

1.3 Bridging Reference Resolution . . . . . . . . . . . . . . . . . 6

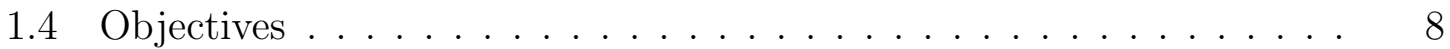

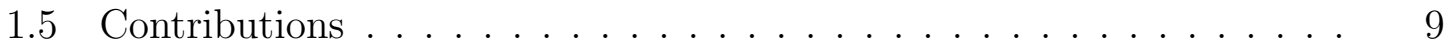

1.5.1 List of Main Contributions of the Present Dissertation . . . . . 10

1.6 Research Questions ....................... 11

1.7 Publications . . . . . . . . . . . . . . . . . . 12

1.8 Thesis Structure . . . . . . . . . . . . . . . . . 12

1.9 Summary . . . . . . . . . . . . . . . . . . . . . . 13

2 Background $\quad 14$

2.1 Automatic Terminology Extraction . . . . . . . . . . . . . . 14

2.1.1 Traditional ATE . . . . . . . . . . . . . . 15

2.1 .2 Machine Learning ATE . . . . . . . . . . . . . 16 
$2.1 .3 \quad$ Distributed ATE . . . . . . . . . . . . . . . 17

2.2 Transfer Learning . . . . . . . . . . . . . . . . . . . . 23

2.3 Domain Adaptation . . . . . . . . . . . . . . . 23

2.4 Statistical Modeling of Language . . . . . . . . . . . . . . . . 27

2.4 .1 Introduction . . . . . . . . . . . . . . 27

2.4 .2 N-Gram Models . . . . . . . . . . . . . . . . . 27

2.4 .3 Vector Space Models . . . . . . . . . . . . . . . . 29

2.4 .4 Word Embeddings . . . . . . . . . . . . . . . 35

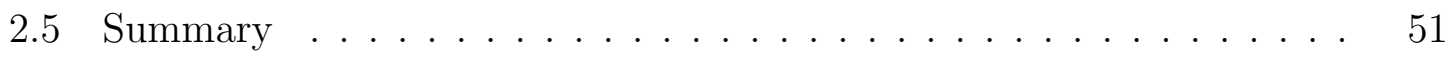

\section{Automatic Terminology Extraction Evaluation: A Gold Standard} for the Mathematics Education Domain 53

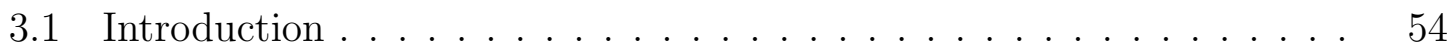

3.2 Related Work . . . . . . . . . . . . . . 56

3.3 Term Extraction Methods and Tools . . . . . . . . . . . 57

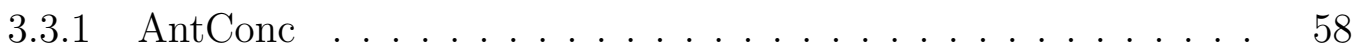

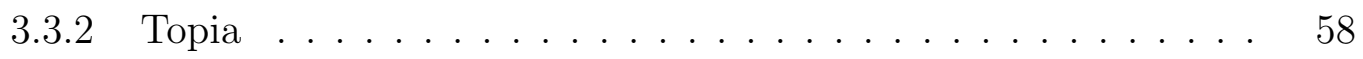

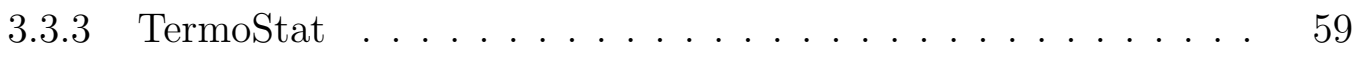

3.4 Sketch Engine . . . . . . . . . . . . . . . . . 60

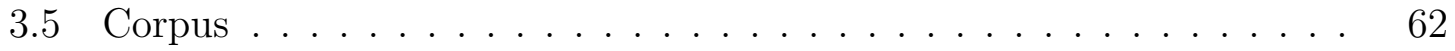

3.6 Term Evaluator . . . . . . . . . . . . . . . . . 62

3.7 The Annotation Process . . . . . . . . . . . . . . . 64

3.8 Results and Analysis . . . . . . . . . . . . . . . 66

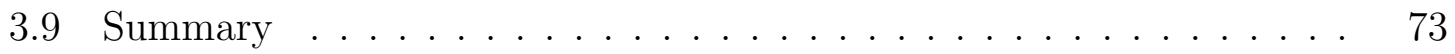


4 Distributed Specificity $\quad 74$

4.1 Introduction . . . . . . . . . . . . . . . . . 75

4.2 Related Work . . . . . . . . . . . . . . . . 78

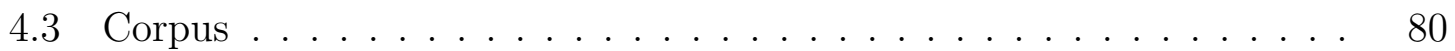

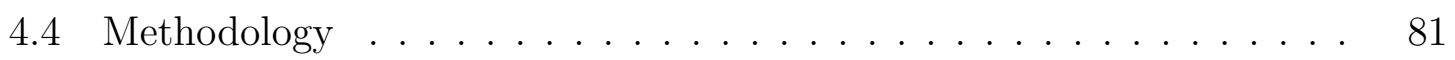

4.4 Specificity Vectors . . . . . . . . . . . . . . . 81

$4.4 .2 \quad$ Filtering Approach . . . . . . . . . . . . . . . . . 84

4.4 .3 Direct Approach . . . . . . . . . . . . . . . 86

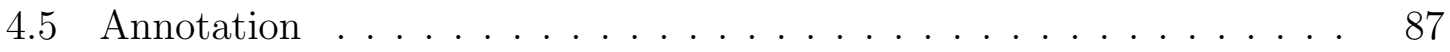

4.6 Experiments and Results . . . . . . . . . . . . . . . 88

4.7 Summary . . . . . . . . . . . . . . . . . . 93

5 Cross-Domain Distributed Automatic Terminology Extraction 94

5.1 Quantitative Experiments . . . . . . . . . . . . . . . 95

5.2 Qualitative Assessments . . . . . . . . . . . . . . . 98

5.3 Domain Adaptation for ATE . . . . . . . . . . . . . . . . . . . 104

5.3 .1 Introduction . . . . . . . . . . . . . . . . . . 104

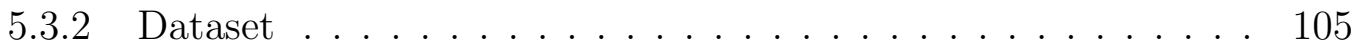

5.3 .3 Methods........................ 106

5.3.4 Experiments and Results . . . . . . . . . . . . . 106

5.4 Summary . . . . . . . . . . . . . . . . . . . 109

6 Distributed Bridging Reference Resolution 111

6.1 Introduction . . . . . . . . . . . . . . . . . . . . . . 112

6.2 Related Work . . . . . . . . . . . . . . . . . . . 117 
6.3 Data . . . . . . . . . . . . . . . . . . . 119

6.4 Methodology . . . . . . . . . . . . . . . . 120

6.5 Experiments \& Results . . . . . . . . . . . . . . . . . . 122

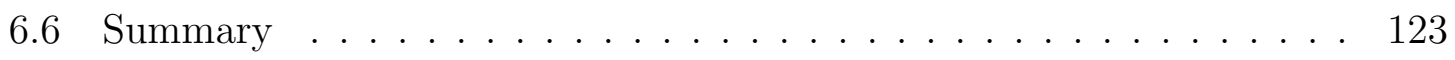

7 Conclusion and Future Research $\quad 124$

7.1 Summary . . . . . . . . . . . . . . . . . . . 124

7.2 Future Work . . . . . . . . . . . . . . . . 129

$\begin{array}{ll}\text { Appendices } & 132\end{array}$

A Full List of Results in Qualitative Assessments 133

A.1 Microbiology . . . . . . . . . . . . . . . 133

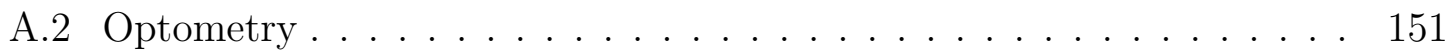

A.3 Economics . . . . . . . . . . . . . . . . . . 175

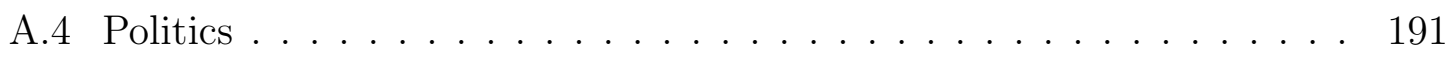

B List of Stop Words Used in Domain Adaptation Experiments as $\begin{array}{lr}\text { Anchor Points } & 204\end{array}$

C List of Stop Words Used in Chapter $3 \quad 206$ 


\section{List of Tables}

3.1 Overall Performance of ATE tools . . . . . . . . . . . . . . . . . . 69

3.2 Inter-annotator Agreement Scores . . . . . . . . . . . . . . . . 71

3.3 Kappa Statistics . . . . . . . . . . . . . . . . . . . . . . . . 72

4.1 Distributed Specificity System Comparison _. . . . . . . . . . . . 91

4.2 Distributed Specificity Direct Approach Results _ . . . . . . . . 92

5.1 Corpora of the New Domains for ATE . . . . . . . . . . . 95

5.2 Sizes of the Datasets for the New Domains . . . . . . . . . . 96

5.3 Training Sets and Test Sets for the New Domains _ . . . . . . . 96

5.4 Distributed Specificity Performance in Microbiology Domain . . . . . 97

5.5 Distributed Specificity Performance in Optometry Domain . . . . . . 97

5.6 Distributed Specificity Performance in Economics Domain . . . . . . 98

5.7 Distributed Specificity Performance in Politics Domain . . . . . . . 98

5.8 Distributed Specificity Qualitative Results in Microbiology Domain . 100

5.9 Distributed Specificity Qualitative Results in Optometry Domain . . . 101

5.10 Distributed Specificity Qualitative Results in Economics Domain . . . 102

5.11 Distributed Specificity Qualitative Results in Politics Domain . . . . 103 
5.12 Domain Transfer Results of Similar Generic words . . . . . . . . . . . 107

5.13 Domain Transfer Results of Dissimilar Words . . . . . . . . . . . . . . 108

5.14 Instances of Unshared Random Word Pair . . . . . . . . . . . . . . . 109

6.1 Syntactic distribution of bridging anchors in our dataset . . . . . . . 119

6.2 Anaphor-anchor lexical relations in our dataset . . . . . . . . . . . 119

6.3 Results of our method (embedding maps) in comparison to the majority class baseline on the dataset . . . . . . . . . . . . . . . 123

A.1 Distributed Specificity Full Qualitative Results in Microbiology Domain 151

A.2 Distributed Specificity Full Qualitative Results in Microbiology Domain 175

A.3 Distributed Specificity Full Qualitative Results in Economics Domain 191

A.4 Distributed Specificity Full Qualitative Results in Politics Domain . . 203

B.1 Stop Words Used as Anchor Points . . . . . . . . . . . . . . . . . 205

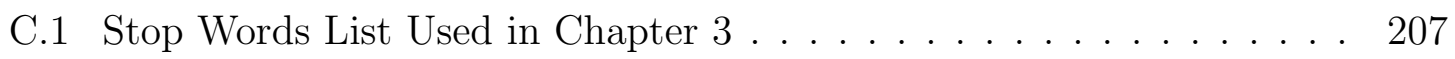




\section{List of Figures}

1.1 Anaphor Pointing to Antecedent . . . . . . . . . . . . . . 7

2.1 CNN Architecture for ATE . . . . . . . . . . . . . . 20

2.2 RNN Architecture for ATE . . . . . . . . . . . . . . . 22

2.3 A Dependency Parse . . . . . . . . . . . . . . . . . . . . 31

2.4 LDA's Graphical Model . . . . . . . . . . . . . . . . . . . . 34

2.5 Neural Probabilistic Language Model Architecture . . . . . . . . . . . . 36

2.6 Recurrent Neural Network Language Model . . . . . . . . . . . . . . . . 44

2.7 eXtended Recurrent Neural Network Language Model . . . . . . . . . . 45

2.8 Continuous Bag Of Words Model . . . . . . . . . . . . . . . . . . 48

2.9 Continuous Skip-gram . . . . . . . . . . . . . . . . . . . . . . . 49

3.1 Term Evaluator Annotation Window . . . . . . . . . . . . . 64

3.2 Term Evaluator Annotation List View . . . . . . . . . . . . 65

3.3 Term Evaluator Annotator Comparison Window . . . . . . . . . . 66

3.4 1-Word Term Evaluation Results . . . . . . . . . . . . . . 67

3.5 2-Word Term Evaluation Results . . . . . . . . . . . . . . 69

3.6 3-Word Term Evaluation Results . . . . . . . . . . . . . . 70 
3.7 4-Word Term Evaluation Results . . . . . . . . . . . . . . 70

4.1 Distributed Specificity Filter System Architectures . . . . . . . . 86

4.2 Distributed Specificity CBOW Classifiers Performance . . . . . . . . 89

4.3 Distributed Specificity Skip-Gram Classifiers Performance . . . . . . . 90 


\section{Chapter 1}

\section{Introduction}

\subsection{The Tasks to Tackle}

Statistical Natural Language Processing (henceforth sNLP) is a subfield of Computer Science and Artificial Intelligence that employs statistics and machine learning at its core to enable machines to model, understand, and generate natural language. The term natural language is used to refer to languages used by humans in their verbal communication, and are distinct from artificial languages such as formal and programming languages. However, understanding natural language is not a trivial task. Languages comprise of multiple layers of encoding (such as phonemes, morphemes, sentences, utterances, meaning, and pragmatics), each layer having a rich structure. Inevitably, understanding natural language boils down to performing numerous tasks at each layer. Despite such complexity in natural languages, humans use (almost ${ }^{1}$ ) only their mostly cortical (Birte U. Forstmann, 2015) network of neural connections to process them. It is efficient to adopt the same device to perform multiple complex

\footnotetext{
${ }^{1}$ Factoring out the involvement of the ears, eyes, and vocal tract.
} 
tasks. Similarly, in sNLP, it would be economical to have one unified representation of some aspects of language that can address different linguistic tasks. For the present study, we will apply such a unified representation, namely, word embeddings, to address the Automatic Terminology Extraction task (refer to section 1.2 for more details about this task) as well as the bridging reference resolution task (refer to section 1.3 for more details about this task).

These two tasks are important subtasks of Information Extraction (IE). IE is the field that applies sNLP techniques to unstructured but machine readable data in order to turn them into structured and usable information. In addressing these tasks, we confine our mode of linguistic messages to text, and to create our representation, we use recent machine learning algorithms based on neural networks with efficient shallow architectures (shallow networks and word embeddings are discussed in section 2.4.4 of this dissertation).

Previous algorithms for these tasks involve various incongruent representations, often each addressing only one aspect of language. These representations are limited in information; for instance, lexical units may refer to the same entity across languages but symbolic representation of those lexical units do not hold such information (e.g., horse, cal, pferd, and hestur). Furthermore, languages contain a multitude of technical domains, and labeled data is rare for most of these domains. Continuous distributed representations can enable transforming a distribution to another and leverage the shared information between different distributions. The present study is the first effort in developing algorithms based on a unified distributed representation for the tasks of automatic terminology extraction and bridging reference resolution. In addition, this study is the first to address domain adaptation for the task of automatic terminology 
extraction.

\subsection{Automatic Terminology Extraction (ATE)}

Automatic Terminology Extraction (ATE) is a subtask of information extraction whose goal is to automatically extract relevant terms from a given corpus (Vu et al., 2008). For instance in a mathematics book, the word apple is not be considered a term but the phrase quadratic equation is. The terminology of a domain encodes the existing knowledge in that domain. Hence, understanding and interpreting a message belonging to a domain cannot be achieved without knowing its terminology. This makes ATE an important task in sNLP. ATE also benefits other tasks in sNLP. The extracted terms by a terminology extraction algorithm are used in a variety of sNLP applications including Information Retrieval (IR), text mining, document summarization, machine translation, parsing sublanguages, question-answering, and ontology construction. The present dissertation applies rich distributed representations acquired by neural networks (refer to section 2.4.4 for further details) to the task of automatic terminology extraction. In doing so, we offer the design of algorithms that treat words and term candidates as non-atomic ${ }^{2}$ and as a distributed ${ }^{3}$ real-valued $^{4}$ representations in the form of a vector. This approach contrasts with traditional and recent algorithms employed for automatic terminology extraction where the terms are treated as atomic elements (Frantzi et al., 1998; Park et al., 2002; Drouin, 2003; Chung and Nation, 2004; Yoshida and Nakagawa, 2005; Vu et al., 2008; VRL, 2009;

\footnotetext{
${ }^{2}$ Irreducible to smaller components

${ }^{3} \mathrm{~A}$ distributed representation presents the information in multiple processing units which often work in parallel

${ }^{4}$ Real-valued components can store more information than binary ones and may provide finer details than discrete or integer values
} 
Yang et al., 2010; Zervanou, 2010; Broß and Ehrig, 2013; Conrado et al., 2013).

Previous terminology extraction methods regard words in a document as atomic elements; that is, they are manifested as their symbolic alphabetical form in the algorithm (such as in (a) below) and/or as some measure of their relative frequency (as in (b) below). Algorithms that have a representation similar to example (a), rely on the word's part of speech, as well as the part of speech of the words surrounding it (or some patterns thereof) to decide if a word is indeed a term. These methods are called linguistic methods ${ }^{5}$. On the other hand, methods that use a representation similar to that shown by example (b) pick a word in the corpus ${ }^{6}$ then count its frequency in the corpus against the size of that corpus, and compare this relative frequency to the relative frequency of the same word in a general corpus containing documents with as many topics as possible. They use this statistical comparison to determine the likelihood that a given word is a term in their target corpus. It is worth noting that when we discuss representation, we are addressing the representation that immediately affects the termhood decision that an algorithm makes. It is also worth mentioning that there are hybrid methods that combine representations exemplified by (a) as well as (b) in their algorithms.

In our approach, each word has tens or hundreds of components and each component is stored at a position in the vector corresponding to that word (as in (c) below). The idea is that finer-learnt granularity may grant more access to the information that a word contains, resulting in a better detection of terms in a document. The type of information that these vectors contain are inherently distributional, although they may be gathered by different methods. In other words, a vector, at a very basic

\footnotetext{
${ }^{5}$ Sometimes referred to as rule-based methods.

${ }^{6}$ The corpus for which they aim to extract its terminology
} 
level, contains the information of which words, in some way or another, co-occur with the word being represented. These co-occurring words of the represented words are often referred to as the word's context (Bengio et al., 2003; Mikolov et al., 2013a; Pennington et al., 2014). The components of the resultant vector are real values that are formed by means of compressing and storing all the linguistic contexts ${ }^{7}$ that the represented word has occurred in. Depending on the characteristics of the specific model, these vectors can preserve sequential, syntactic, and/or semantic information (Bengio et al., 2003; Mikolov et al., 2010, 2013f; Pennington et al., 2014). These vectors are built to create a high-dimensional space such that the components of the vectors in that space can be automatically tuned to maximize the likelihood of correctly predicting a next occurring word given the preceding ones or, alternatively, to best differentiate words that are distributionally different and group together those that are distributionally similar, despite keeping the information pertaining to their subtle differences. Therefore, the components of these vectors can be thought of as containers to hold specific encoded information upon some form of processing (or reasoning, if you will) of linguistic data. This can be achieved in various ways (see section 2.4.3), but we use the most efficient methods comprising shallow neural networks that observe the words in their contexts and encode them into vectors. In section 2.4, we discuss in detail the statistical models of (aspects of) language and the reasons for our choice of architectures.

(a) apple, NOUN

(b) 0.0003654

(c) $[0.54407,0.9233,0.50644,0.46454,-0.62015,-0.35166, \ldots-0.93253]_{n}$

\footnotetext{
${ }^{7}$ Or cotext, to be linguistically accurate
} 
Where $n$ is often between 50 to a thousand for neural network word embeddings. The value of $n$ is optimized given the size of training data, efficiency of the algorithm, available computational resources, and the learning capacity of the architecture of the model.

\subsection{Bridging Reference Resolution}

Reference resolution is another subtask of information extraction which aims to determine what linguistic expression refers to what entity in the text. This reference involves an anaphor and an antecedent, where the former, typically, but not always, follows the latter, and, in some way, points back to it (see figure 1.1 for an illustration). For instance, in the utterance "Cyrus talked to Mary today about why he failed an exam" he is the anaphor and Cyrus is the antecedent of that anaphor. Among all the entities mentioned in this very simple case (i.e., Cyrus, Mary, and exam), he refers to the same entity as Cyrus does in the world. Linguistically and semiotically speaking, they are two different signifiers of the same signified (de Saussure, 1983). The signified, which also may be referred to as entity in sNLP, is unique in the context (or "world", if you will) where the reference takes place. Example (1) below presents different types of references.

Utterance $a$ in example 1 below is an instance of coreference where the anaphor refers to the same entity as its antecedent does, that is, "He" is referring to the same person that "Cyrus" is. If an anaphor fails to refer to an antecedent, it gives rise to a phenomenon called reference failure and as a result the text loses its cohesion, as can be observed in utterance $b$. In utterance $c$, however, the anaphor (i.e., "the waiter") has no antecedent but the utterance does not suffer from reference failure. It 
Figure 1.1: Typically an anaphor follows its antecedent and points back to it. The straight lines in the figure signify the text in between the two.

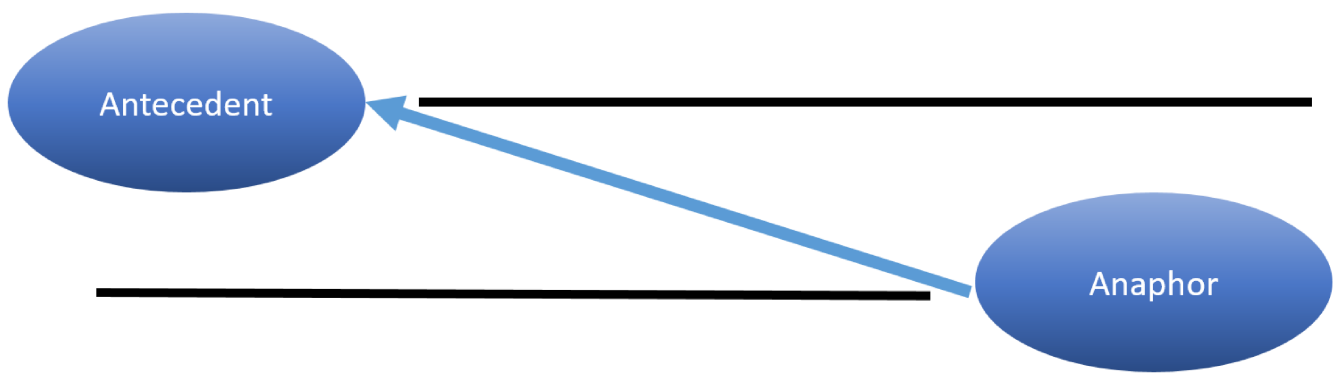

evades such reference failure by bridging to another entity present in the discourse, in particular, "that restaurant". As a result, the entity of "the waiter" is inferred by the hearer of the utterance to be "a waiter in that restaurant". In such scenarios, entities such as "restaurant" that are bridged to by bridging anaphors are called anchors. This specific type of reference is often called (non-coreferent) bridging reference, and the process that a hearer undergoes to make the bridge and infer the anchor is called accommodation. (Clark, 1975; Poesio et al., 1997; Hou et al., 2013a). Following Hou et al. (2013a,b), we call the two mentions involved in a bridging reference as (bridging) anaphor and anchor, as opposed to anaphor and antecedent.

a. I talked to Cyrus yesterday. He was upset.

b. Mary called. He is tall.

c. We went to that restaurant last night; the waiter was really nice.

The second goal of the proposed research is to devise an automated system that can identify such bridges that veto reference failure, and pair bridging anaphors with their 
anchors. Resolving a bridging reference is a complex cognitive task that involves finding the correct anchor for an anaphor, in addition to detecting the bridging anaphor itself. This dissertation offers the design and implementation of an algorithm that identifies bridging anaphors and their correct anchors in bridging references using distributed representations that are acquired by neural networks. This representation is the same as the one we employ for the task of automatic terminology extraction.

\subsection{Objectives}

Below we list the main objectives of the present thesis.

1. Improve the performance of the automatic terminology extraction algorithms

2. Improve the representation in ATE and bridging reference resolution

3. Transfer information about termhood from one technical domain to another in automatic terminology extraction

4. Make few-shot learning and minimal supervision available to ATE and enabling ATE algorithms to learn using a few labeled datapoints

5. Provide a dataset for automatic terminology extraction in the mathematics education domain

6. Provide a dataset for the task of bridging reference resolution

7. Discard the need for human annotation in supervised ATE

8. Take a step forward towards abolishing the need for feature engineering in ATE and Bridging Reference Resolution 
It is critical to note that successfully applying a unified distributed representation to multiple tasks in information extraction (i.e., automatic terminology extraction and bridging reference resolution) opens the door for transfer learning, not only across domains, but also across all the tasks in information extraction, in addition to the feasibility obtained from a unified and homogeneous method to obtain a rich representation for the tasks.

\subsection{Contributions}

Our study is the first to employ a unified connectionist ${ }^{8}$ approach.

The present work substantially improves on earlier ATE algorithms, enables minimal supervision for new technical domains, makes available the implementation of supervised algorithms with no human annotation required, and puts forth the potential of domain adaptation for automatic terminology extraction.

Employing a continuous distributed representation is a step forward towards adopting a single unified representation to perform multiple sNLP tasks and is another contribution of the present study which connects ATE and bridging reference resolution to all the other tasks in modern NLP. A more concrete consequence of this method is a) having a more accurate terminology extraction algorithm and b) abolishing the need for feature engineering ${ }^{9}$ and expert knowledge for annotating data which would be limited to a particular language and domain. Furthermore, implementing any new algorithm to any new domain requires novel methods and solutions

\footnotetext{
${ }^{8} \mathrm{~A}$ connectionist approach uses a distributed architecture (i.e., neural networks) and a distributed representation (e.g., the so called word embeddings), as opposed to other alternatives, such as the symbolic (rule-based) approach

${ }^{9}$ The process of manually selecting the potentially significant features in the data by an expert, which are deemed fruitful for the task.
} 
to be devised to address various subtleties involved in performing the tasks, and such novel methods and solutions offered in this dissertation are yet another contribution of the present study.

Both the representation and the processing used by the offered algorithms are more similar to the representation and processing that take place in the human brain than any other previous approach, since our algorithms devise a neural network architecture and observe nothing but raw text (i.e., one mode of human language) as it is, along with some examples, to reach an understanding of the phenomena at hand.

This study developed a gold standard dataset in order to apply ATE to the educational mathematics domain and benchmark the previous methods, as well as a novel method for ATE based on distributed representations. It also offers a new dataset for bridging reference resolution where the original definition of bridging anaphora is followed (see section 6.1 for further details) while covering a diverse set of bridging relation instances. Furthermore, the present study shows that the distributed representations can also be utilized for the task of bridging reference resolution.

\subsubsection{List of Main Contributions of the Present Dissertation}

Below is the list of the contributions of the present dissertation:

1. Applies a novel connectionist approach to address the task of ATE

2. Applies a novel connectionist approach to address the task of bridging reference resolution

3. Substantially improves on earlier ATE algorithms

4. Enables minimal supervision for ATE 
5. Offers a new dataset that follows the original definition of bridging anaphora and contains a diverse set of bridging relations

6. Discards the need for human annotation in supervised ATE altogether

7. Addresses domain adaptation for ATE for the first time

8. Abolishes the need for feature engineering in ATE and Bridging Reference Resolution

9. Offers a gold standard dataset for the mathematics education domain

10. Applies and evaluates ATE on multiple technical domains

\subsection{Research Questions}

Given the premises stated above, the present research seeks to provide answers to the following research questions:

1. Can rich distributed representation acquired by neural networks be leveraged to improve automatic extraction of terminology?

2. Can an algorithm employing such a representation succeed with minimal supervision?

3. Can such the algorithm be applied to other domains without the need for human annotation?

4. Can rich distributed representations acquired by neural networks be leveraged to address the bridging reference resolution task? 


\subsection{Publications}

Below are the publications that the present thesis has contributed to:

1. Amjadian, E., Inkpen, D., Paribakht, T., S., and Faez, F. (2018), "Distributed Specificity for Automatic Terminology Extraction". Terminology 24(1), 2018. Amsterdam: John Benjamins.

2. Amjadian, E., Inkpen, D., Paribakht, T., and Faez, F. (2016). Local-global vectors to improve unigram terminology extraction. In Proceedings of the 5th International Workshop on Computational Terminology (Computerm2016), pages 2-11, Osaka, Japan. The COLING 2016 Organizing Committee.

3. Inkpen, D., Paribakht T. S., Farahnaz F., and Amjadian, E. 2016. Term Evaluator: a tool for terminology annotation and evaluation. International Journal of Computational Linguistics and Applications, 7(2): 145-165.

4. Abid, M. R., Petriu, E. M., and Amjadian, E. (2015). Dynamic sign language recognition for smart home interactive application using stochastic linear formal grammar. IEEE Trans. Instrumentation and Measurement, 64(3):596-605.

\subsection{Thesis Structure}

Chapter 1 introduced the tasks this thesis strives to address, and briefly stated the incentives for a method based on which our algorithms are designed throughout the study. Chapter 2 provides the required background a) in prominent methods in modeling natural language, resulting in the modern continuous distributed representations that our study is based on, b) in the task of automatic terminology extraction and 
how it has been approached before, c) in transfer learning and domain adaptation. Chapter 3 offers a thorough analysis of the previous ATE methods and evaluates them in a specific domain. Chapter 4 presents our novel algorithm that substantially enhances the efficacy of ATE with minimal supervision. In chapter 5 we explore the application of our algorithm to other domains and investigate the potential of domain adaptation for ATE. Chapter 6 applies distributed representations to the task of bridging reference resolution. Finally, chapter 7 concludes the study.

\subsection{Summary}

In this section of the thesis, we first mentioned the goal of the proposed study (section 1.1) that is to devise novel algorithms to address and advance two core task in information extraction, namely, automatic terminology extraction (section 1.2) and bridging reference resolution (section 1.3). The objectives of the thesis were stated afterwards(section 1.4). Then the major contributions of the study were put forth (section 1.5) and the research questions were presented (section 1.6). Section 1.8 presented the structure of the current thesis. 


\section{Chapter 2}

\section{Background}

This section reviews the literature related to the goals of this thesis. We start with the literature for ATE. Then will provide a history of transfer learning and domain adaptation. Finally, we will take a close look at methods to represent language and their evolution to shed light as to why the specific distributed representation was chosen for this study and to serve as the background information for the later chapters.

\subsection{Automatic Terminology Extraction}

This section discusses the literature on ATE. We divide ATE studies into three streams, traditional, machine learning, and distributed ATE, as described below.

1. Traditional ATE employs linguistic and statistical methods to address ATE.

2. Machine learning ATE relies on feature engineering and supervised machine learning algorithms 
3. Distributed ATE utilizes distributed representation and deep learning techniques.

\subsubsection{Traditional ATE}

ATE approaches traditionally fall into three categories, namely, linguistic, (unsupervised) statistical ${ }^{1}$, and hybrid methods (Cabre-Castellvi et al., 2001; Chung, 2003). These ATE methods have been applied to both monolingual and multilingual corpora (Ljubešić et al., 2012). Linguistic methods apply hand-coded rules to the target corpus to extract technical terms. They often leverage syntactic patterns that are indicative of occurrence of a term. Statistical methods are often unsupervised and apply some measure of relative frequency to a technical target corpus, a reference (general) corpus and sometimes a (contrastive) corpus from another domain, to identify the existing terms in the technical corpus (Frantzi et al., 1998; Chung, 2003; Vu et al., 2008; Conrado et al., 2013). These methods are often referred to as specificity methods as they recognize a term by how specific it is to the target corpus as compared to a general domain reference corpus. They are computationally efficient and are still prevalent in today's search engines and information retrieval systems. Purely statistical methods are language independent, that is, they can be readily applied to any non-polysynthetic language. In polysynthetic languages, such as Turkish, words are less isolated and have a richer morphology and affixation mechanism which requires a separate tokenization procedure often absent in vanilla statistical methods. Hybrid methods combine statistical and linguistic methods to extract terminology from a target corpus and often perform very well (Drouin, 2003; Le Serrec et al.,

\footnotetext{
${ }^{1}$ These statistical methods are distinct from statistical learning approaches.
} 
2010; Ismail and Manandhar, 2010; Vintar, 2010). The linguistic rule-based component in hybrid approaches often addresses the extraction of multi-word term phrases. The above-mentioned approaches, in contrast to the methods put forth in this paper, regard words as atomic units represented by their linguistic forms and their statistical scores that indicate the likelihood that they are terms. They may, however, implement rules associated with some linguistic features (e.g., their POS tags, their position in the POS sequence, their position in the parse tree, phrase, and/or in the sentence). These linguistic rules make an algorithm language-dependent and even sometimes to some degree domain-dependent. On the contrary, our method, if used independently, can be applied to any non-polysynthetic language and for any domain as long as a suitably-large domain-specific and general corpora are available. In this study, we apply our method in combination with other methods as well as independently. It can be regarded as a step towards a language independent ATE algorithm that benefits from latent linguistic information encoded in the vectors used (see section 2.4 for further details on vector space models and word embeddings) in comparison to purely statistical methods that do not capture such information.

\subsubsection{Machine Learning ATE}

Supervised methods have been implemented for terminology extraction. Nazar and Cabré (2012) used examples of terms from the domain of interest and a reference corpus of general language, which represent positive and negative examples of terms, and a three-level (i.e., syntactic, lexical, and morphological) learning algorithm to detect the terms. They used the frequency distribution for POS tag sequences at the syntactic level. At the lexical level, they accounted for the frequency of the lexical 
units within the terms (word forms, as well as lemmas). Finally, at their morphological level, from each word type they extracted initial and final character $n$-grams where: $1 \leq n \leq 5$ (Nazar and Cabré, 2012). Their term extractor is an online web-based system that is constantly being updated when used by terminologists. More recently, Conrado et al. (2013) achieved state-of-the-art performance for unigram term extraction in Brazilian Portuguese using supervised learning algorithms and a rich feature set. They used eight linguistic features, seven statistical features, and four hybrid features in their method. There has, however, been earlier supervised work in keyword/keyphrase extraction such as Turney (2000), as opposed to terminology extraction which is the topic of the present work. While keyword extraction is the task of extracting only a few keywords in a text, terminology extraction aims to detect the terms, usually from a large domain corpus. ATE is deemed more challenging due to many reasons including the fact that it requires more than a high-precision specificity computation to function agreeably.

\subsubsection{Distributed ATE}

The present work is the next phase for these supervised methods, since we move closer to a rich, language-independent, resource-independent, and fully data-driven representation. It is worth noting that modern word embeddings have been successfully employed in many tasks, including the related areas of keyphrase extraction (Wang et al., 2015) and aspect term extraction ${ }^{2}$ (Yin et al., 2016). In the present study they are leveraged for the general terminology extraction task.

\footnotetext{
${ }^{2}$ Aspect term extraction is the task to identify the aspect expressions which refer to a product's or service's properties or attributes, from customer reviews (Pontiki et al., 2014, 2015; Yin et al., 2016).
} 
Amjadian et al. (2016) and Wang et al. (2016), in independent studies, were the first to apply distributed representation and deep learning to the task of automatic terminology extraction. Both of these studies eliminate the need for feature engineering for ATE. Feature engineering is the task of designing and extracting informative features for a certain task relying on domain expertise of human experts. Such a process often makes the machine learning algorithms domain dependent. As for ATE one of the side effects of feature engineering is that it often makes the algorithm language dependent, since the engineered features may rely on features that are specific to a certain language.

Amjadian et al. (2016) and Wang et al. (2016) also both adopt architectures put forth by Mikolov et al. (2013a) and Mikolov et al. (2013e). However, Wang et al. (2016) only employ the skip-gram architecture, whereas Amjadian et al. (2016) investigate both the skip-gram and $\mathrm{CBOW}$ architectures. For a detailed account of these architectures see section 2.4.4. Wang et al. (2016) employ $\mathrm{CNN}^{3}$ and $\mathrm{RNN}^{4}$ architectures (described below) whereas Amjadian et al. (2016) utilizes a feed forward architecture. Both of these studies substantially improve over previous methods. Amjadian et al. (2016) targets a constructed math domain corpus, whereas Wang et al. (2016) use GENIA ${ }^{5}$ and ACL RD-TEC ${ }^{6}$ that are biomedical domain and computer science domain respectively.

Both of these studies address the challenge of labeled data scarcity. Wang et al. (2016) employ a semi-supervised bootstrapping method called co-training which picks the prediction of the most confident classifier from a pair of classifiers in order to

\footnotetext{
${ }^{3}$ Convolutional Neural Network

${ }^{4}$ Recurrent Neural Network

${ }^{5}$ The GENIA corpus can be accessed from: http://www.geniaproject.org/genia-corpus

${ }^{6}$ The ACL RD-TEC can be accessed from: https://github.com/languagerecipes/the-acl-rd-tec
} 
add to the labeled data. Amjadian et al. (2018), in contrast, devise the distributed specificity representation, vectors that take into account domain specific as well as general characteristics of a word, which is highly indicative of the state of termhood of that word. These two methods also contrast in the number of (seed) training data required. Wang et al. (2016) require 200 seed datapoints, whereas Amjadian et al. (2016) can start with only 9 . This is a critical difference, as requiring only 9 labeled datapoints practically eliminates the need for manual annotation altogether in term extraction, such that a classical specificity algorithm can be used with high-precision settings to acquire the few labeled datapoints required for training. Wang et al. (2016) tackle both unigram terms as well as multi-word term phrases, although, the term phrases are extracted by stop word delimiters and are only approved or discarded by the offered algorithm in a binary classification. This approach leaves the detection of many phrases up to chance. Amjadian et al. (2016) focus on improving unigram terms only, given the specifics of their study, particularly, due to a high performance of one of the existing systems they tested (refer to chapter 3 for further details) on multi-word terms as compared to unigram terms.

The methods introduced and experiments carried out by Amjadian et al. (2016) are the topic of chapter 4 and will be discussed there in detail. The details of the method used by Wang et al. (2016) will be discussed further below.

Wang et al. (2016) co-train 2 deep learning architectures to compete in making the predictions for the unlabeled datapoints. In their CNN architecture, term phrase candidates are transformed to their corresponding embeddings, then a few regions are constructed based on n-gram combinations of the input. These regions of embeddings are fed to the CNN, each going through a corresponding set of filters designed to fit 
the size of the region. The resulting feature maps then go through a max pooling layer and are passed to the logistic layer for binary classification afterwards.

Figure 2.1: The figure illustrates the convolutional neural network architecture by Wang et al. (2016). The regions select different n-grams from the phrase and pass them on to their designated filters. The symbol $r$ represents the number of regions, $n$ represents the number of filters in each region, $d$ is the dimensionality of the embeddings, and $L$ is the length of the term phrase in words. The final logistic layer makes the final call.

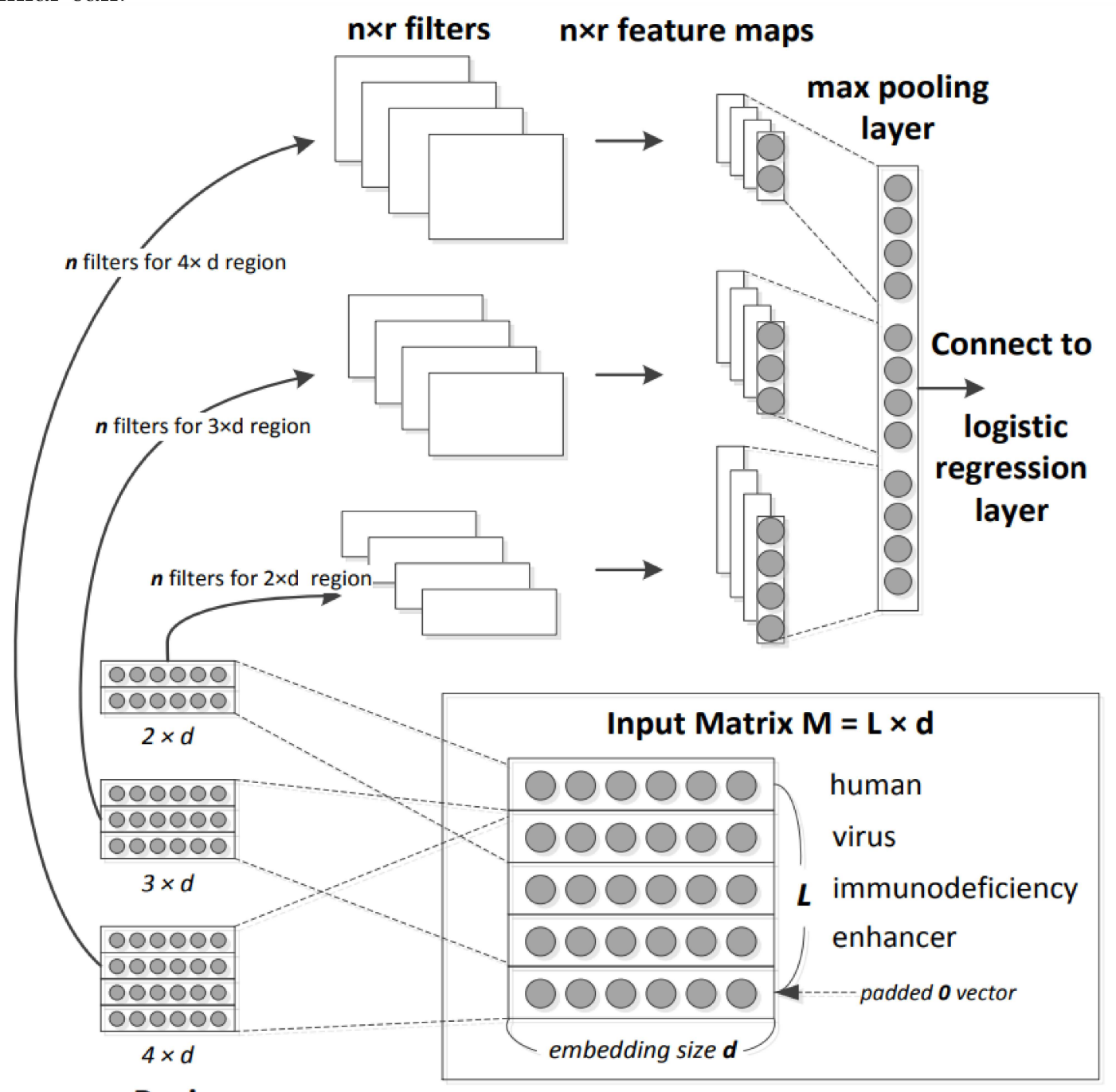

r Regions 
Their second competing classifier is an $\mathrm{LSTM}^{7}$ which is one of the most prominent RNN architectures. The LSTM receives the words embeddings $x_{t}$ one by one in a sequence, along with the hidden state of its previous time-step $h_{t-1}$, constructs the forget, input, and output gates $\left(f_{t}, i_{t}\right.$, and $o_{t}$ respectively) using this information as well as their corresponding weights (i.e., $W$ 's and $U$ 's) and biases (i.e., $b$ ). The forget gate $f$ decides what information from the memory cell of the previous time step $c_{t-1}$ remains in the memory cell in the current time step $c_{t}$, whereas the input gate $i_{t}$ determines what information from the new input, that is, candidate memory cell $g_{t}$, is retained as part of the new memory cell $c_{t} . g$ is the hyperbolic tangent of a linear combination of the input $x_{t}$ and the hidden state from the previous time step $h_{t-1}$. Finally the output gate $o_{t}$ decides what information, from the current constructed memory cell, gets passed on to the hidden state of the current time step $h_{t}$. The final hidden state $h_{\text {final }}$ is passed to a logistic layer to decide of the input candidate term phrase is a term. From each prediction the two classifiers, namely, CNN and RNN make, the one with a higher confidence score is accepted as the label for the term phrase candidate.

Finally Gharbieh et al. (2017) explored the application of deep learning techniques $^{8}$ and distributed representation to identify multi-word expressions. Multi-word expressions are not necessarily terms and are in that regard irrelevant to ATE. They are phrases that manifest a unified structure and are idiomatic in nature. They range from noun phrases and phrasal verbs, to named entities and proverbs (Gharbieh et al., 2017). Nevertheless, it is worthwhile to note that deep learning methods can perform

\footnotetext{
${ }^{7}$ Long Short-Term Memory

${ }^{8}$ specifically, CNN, RNN, and feed forward architectures
} 
Figure 2.2: The figure illustrates the long short-term memory architecture adopted by Wang et al. (2016). Description $\oplus$ signifies a vector addition where $\otimes$ indicates element-wise vector multiplication. There are two types of activation functions in the diagram, namely, sigmoid and hyperbolic tangent. The last hidden state is passed to a logistic layer for binary classification.

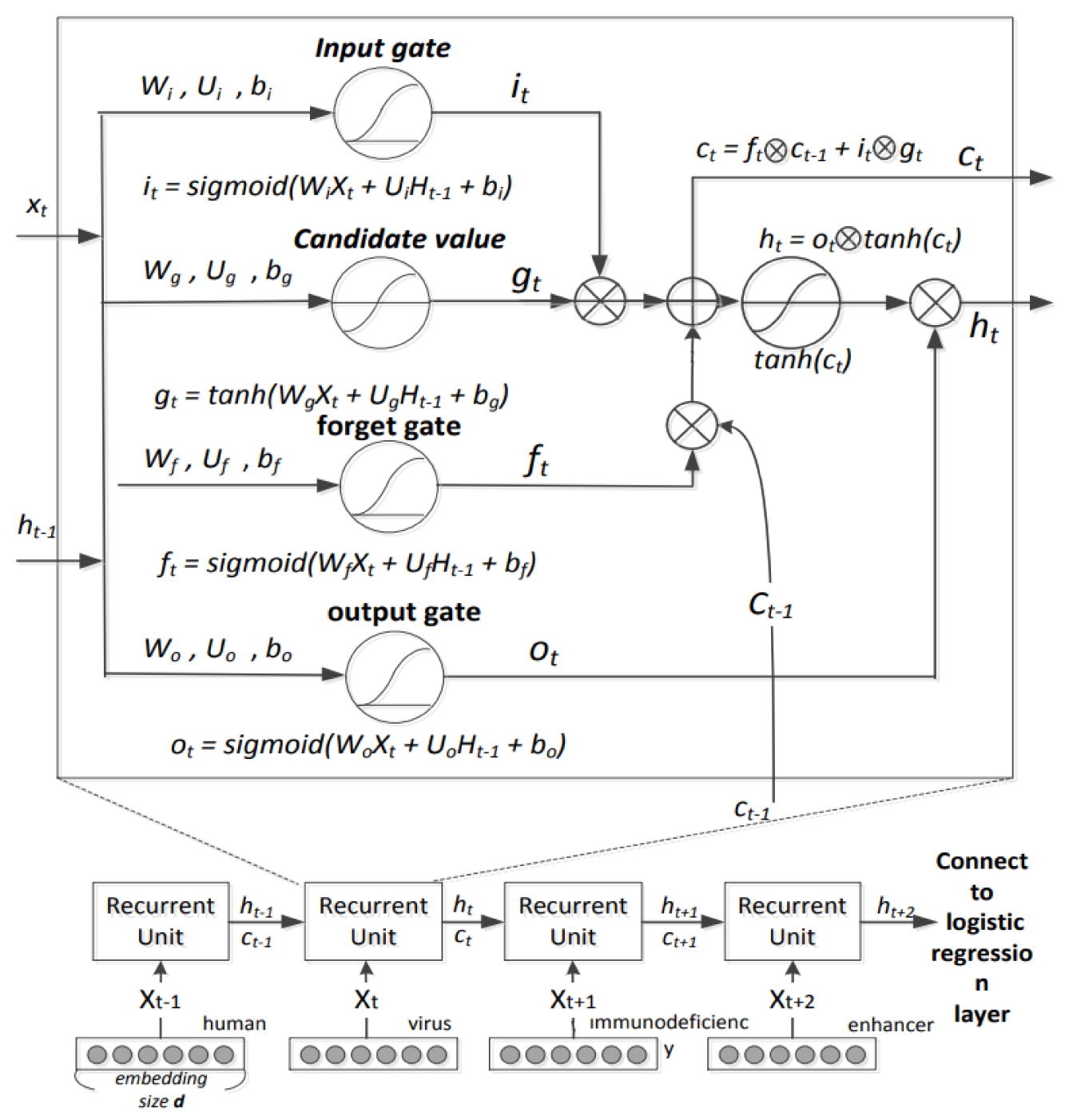

well to detect unithood ${ }^{9}$ in multi-word expressions which is a property shared with multi-word term phrases. One notable disadvantage of the method applied by Ghar-

\footnotetext{
${ }^{9}$ Unithood is defined as the degree of stability of a syntagmatic combination(Kageura and Umino, 1996)
} 
bieh et al. (2017) is that it requires substantially more training data than utilized by the methods put forth by Wang et al. (2016) and Amjadian et al. (2016), and it offers no strategy for weak supervision.

\subsection{Transfer Learning}

Transfer Learning (TL) is a subfield of artificial intelligence and machine learning that involves transferring knowledge learned to perform one task to another. This transferred knowledge may either suffice in performing the second task or help facilitating learning the task. Deep learning has benefited extensively from TA mostly in the shape of reducing the amount of training data needed for the tasks, relying on the patterns learned about similar data while performing another task. One of the most common forms of TL in sNLP is the employment of (word) embeddings. Many tasks in sNLP rely on the dense representations learned for words, characters, and sometimes phrases to inform their algorithms of the patterns discovered by processing large corpora of text. They are as diverse as machine translation (Choi et al., 2018), text summarization (See et al., 2017), and task-oriented dialog systems (Zhong et al., 2018) among many other sNLP tasks.

\subsection{Domain Adaptation}

Domain Adaptation (DA) is a special case of transfer learning, that addresses transferring knowledge from one domain to the other, often concerning the same task (Csurka, 2017). DA is specifically useful when we know or can assume that the source and target data are drawn from different distributions where the performance 
of a machine learning algorithm trained on one domain degrades on the other. This difference in the distributions of two or more domains is sometimes referred to as domain shift (Csurka, 2017). To the best of my knowledge DA has not been applied to ATE. However, it has been applied to the related task of bilingual mapping of word embeddings which is a substantial source of information for many prevalent statistical machine translation systems.

It is common in machine learning not to have any training data for a task or a specific domain of a task. For instance, one may have labeled spam and non-spam emails for the spam filtering task in one language but not another. Mikolov et al. (2013d) were the first to employ distributed representation to map from embeddings in one language to another. Their task was creating a bilingual lexicon given monolingual vectors. Earlier methods made use of similarities in the morphology between a pair of related languages or alternatively matching contexts between the two to detect potential translations (Haghighi et al., 2008). These morphological methods, however, were not effective for language pairs that are not as related for instance EnglishChinese or Czech-English (Mikolov et al., 2013d).Mikolov et al. (2013d) noticed that a set of words in one language (one to five) that are in the same relationship with each other as their counterparts are in another language (uno to cinco) are arranged in similar geometries, when visualized using $\mathrm{PCA}^{10}$. AS a result the offered a linear mapping between embeddings of different languages to preserve the above-mentioned geometry, optimizing the equation below.

$$
\min _{W} \sum_{i=1}^{n}\left\|W x_{i}-z_{i}\right\|^{2}
$$

where $W$ is the transformation matrix and contains the learnable parameters, $x$ is

\footnotetext{
${ }^{10}$ Principal Component Analysis
} 
the source language embeddings vector and $z$ is the paired target language word vector. At inference they use the cosine similarity to locate the nearest neighbor of the transformed vector in order to predict the pair. They use the CBOW architecture (see section 2.4.4 for further details about this architecture) to generate their distributed representation.

Recently, Joulin et al. (2018) devised a new method to transform the embedding distribution from one language to another which generalizes well to unseen words. Their goal was to learn a linear mapping between the two distributions. They state that the orthogonal constraint in orthogonal Procrustes analysis may be prone to discarding critical information about the distributions, and show that comparable results can be achieved without such a constraint. The orthogonal constraint requires the transformation matrix $W$ to be orthogonal as defined below.

$$
W^{T} W=I_{d}
$$

The orthogonal constraint is to keep the distance between embeddings constant under transformation in order for the target language embeddings to manifest relationships similar to that of the source embeddings.

The task that Joulin et al. (2018) took on was a paired domain adaptation task in nature, that is, for each datapoint (i.e., a word) in the source domain there exists a counterpart (i.e., a translation) in the target domain. One of the challenges of the paired domain adaptation task in natural language is the hubness problem (Dinu and Baroni, 2014), which observes that some word vectors are the nearest neighbors of a large number of other words. This is problematic for the bilingual mapping task, as it renders the nearest neighbor search for the intended target word inefficient after transformation. 
A solution to the above mentioned problem is to use Inverted SoFtmax (ISF) (Smith et al., 2017) or alternatively Cross-domain Similarity Local Scaling (CSLS) (Conneau et al., 2017). CSLS is described below.

$$
C S L S(x, y)=-2 \cos (x, y)+\frac{1}{k} \sum_{y^{\prime} \in \mathcal{N}_{Y}(x)} \cos \left(x, y^{\prime}\right)+\frac{1}{k} \sum_{x^{\prime} \in \mathcal{N}_{X}(y)} \cos \left(x^{\prime}, y\right)
$$

Where $\mathcal{N}_{Y}(x)$ are the $k$ nearest neighbors of the point $x$ in the set of vectors $Y=$ $\left\{y_{1}, \ldots, y_{N}\right\}$, and $\mathcal{N}_{X}(y)$ are the $k$ nearest neighbors of the transformed point $y$ in the set of vectors $X=\left\{x_{1}, \ldots, y_{x}\right\}$.

In the context of mapping between word embedding distributions the above symmetric equation is regarded as the cost of undesired target embeddings being in the neighborhood of the transformed embedding.

To avoid the resulting discrepancy between the training and inference stages Joulin et al. (2018) offer a relaxed variation of the above equation as the training as well as the inference cost for mapping between pairs of words in two languages.

One of the challenges of employing methods similar to the ones utilized by Mikolov et al. (2013d) and Joulin et al. (2018) is that they address a paired DA task (i.e., bilingual mapping of word embeddings) whereas different domains in ATE are not required to have matching pairs of terms; on the contrary, they predominantly have different sets of terms with little intersection. We will address this problem in section 5 of this dissertation. 


\subsection{Statistical Modeling of Language}

\subsubsection{Introduction}

In our studies, we employ statistical models of language as the basis of our linguistic representation. Many different statistical methods can be used to model natural language. Among them, we employ a method based on neural networks (v.i. section 2.4.4) for our tasks. The present chapter briefly discusses the most popular methods for modeling natural language and states some of their theoretical, mathematical, and computational differences. We will go over these methods in chronological order of their emergence, from the earliest to latest. Adequate understanding of these methods is required in selecting the right one(s) for any given task.

We start this section by briefly mentioning the origin of such models, that is, n-gram models that are the traditional statistical language models. Then we discuss the main types of vector space models, namely, latent semantic analysis and latent Dirichlet allocation. Finally, we will address word embeddings learnt by neural networks.

\subsubsection{N-Gram Models}

Traditional statistical language models, also referred to as n-gram models, have been used abundantly in sNLP. They have been one of the main underlying components of many sNLP applications, including but not limited to automatic speech recognition, statistical machine translation, named entity recognition, speech synthesis, spelling correction, grammar checking, natural language generation, authorship identification, predictive text input systems, and part of speech taggers (Jurafsky and Martin, 2009; 
Manning and Schütze, 1999). They have also been used outside sNLP, for instance, in handwriting recognition (Russell and Norvig, 2003) and recently in computer vision (Abid et al., 2015).

N-grams predict the $n$th word given the previous $n-1$ words. As early as the beginning of the 20th century (i.e., 1913), Markov used them to predict the next occurring vowel or consonant in Eugene Onegin by Alexander Pushkin (Markov, 1913; Jurafsky and Martin, 2009). Formally, an n-gram language model is:

$$
P\left(w_{t} \mid w_{1}^{t-1}\right) \approx P\left(w_{t} \mid w_{t-n+1}^{t-1}\right)
$$

where $t$ is the position of the word that the model is to predict in the text, and $n$ is the number of grams in the language model. For instance, for a bigram language model, the model is trained on and makes predictions based on only the previous word, for a trigram language model, the previous two words, and if it is a 4-gram language model, the model makes predictions based on the information provided by the previous three words.

Another task for which n-gram models are used is to compute the probability of an entire word sequence. Formally:

$$
P\left(w_{1}^{t}\right) \approx \prod_{i=1}^{t} P\left(w_{i} \mid w_{i-n+1}^{i-1}\right)
$$

N-gram models are discrete models where the words are treated as non-reducible atoms $^{11}$. Such discrete models are fast and computationally cheap. They have been a great factor in the advent of state-of-the-art sNLP; nevertheless, they can only predict the occurrence of a next word in a sequence or compute the probability of a sequence of words, whereas in reference resolution for instance, the anaphor and its

\footnotetext{
${ }^{11}$ They cannot be decomposed to smaller components
} 
antecedent may be quite far apart ${ }^{12}$ and hence the mystery does not lie in how likely an entire sequence of a few sentences is, but how likely it is that two elements in such a long string of words pick the same entity in the context. It follows that the model needs to capture some semantic, syntactic, and lexical relations among words (and even phrases), and n-gram models are not designed for such purposes. Furthermore, in terms of computability, natural languages are at least as complex as context-free languages (Chomsky, 1956, 1957).

This fact emphasizes that they have depth as well as breadth. But linear models, such as n-gram models, can only capture the linearity (i.e., the breadth) of a natural language. for tasks in information extraction requiring uncovering not only linear but also non-linear structures in the data, a distributed approach seems more desirable, where the words are not the atomic elements and can be represented by multidimensional numeric vectors. Hence, a word has a geometrically-richer representation than only a linear and sequential probability. This leads us to the next section that discusses vector space models.

\subsubsection{Vector Space Models}

Vector Space Models (henceforth VSMs) are based on the idea that words occurring in similar contexts tend to have similar meanings, as stated by Harris (1954) (Kiela and Clark, 2014). Similarly, Firth (1957) states "You shall know a word by the company it keeps!" (Jurafsky and Martin, 2009). Formally, if the vocabulary size of a language is $\mathrm{n}$, a word in a VSM is a vector of size $\mathrm{n}$ such that:

(6) $\quad \vec{w}=\left(f_{1}, f_{2}, \ldots, f_{n}\right)$

\footnotetext{
${ }^{12}$ This is referred to as long distance dependency and is a challenge for n-gram models
} 
where $\left(f_{1}, f_{2}, \ldots, f_{n}\right)$ are the components of the vector and $f$ denotes a feature. Therefore, words are identified by the values of their features, as opposed to the symbolic representation in n-gram models. The features of the vectors are either of binary (e.g., $(1,0,1))$, frequency (e.g., $(23,92,0))$, or association type (e.g., $(0.20,0.95,0.01))$. A commonly used association measure is Point-wise Mutual Information or PMI for short and:

$$
a_{P M I}(w, f)=\log _{2} \frac{P(w, f)}{P(w) P(f)}
$$

where $w$ is a word and $f$ is a feature. Generally, two approaches have been used to determine the context in which word co-occurrence scores are computed: window based and dependency based (Kiela and Clark, 2014). In the window-based approach, word co-occurrences are computed only in a window of a given size (in number of words), usually with the target word in the center of the window. Hence, only the co-occurrence of the words in that window are counted for the target word. In the dependency-based approach, word co-occurrences are computed only for the words that are in a particular syntactic relationship with the target word. For instance, in a sentence only the verb, its subject, and its object may be considered in a hypothetical dependency VSM (see figure 2.3 for an example of a dependency parse). This is a strong characteristic that enables dependency-based VSMs to model more specific information in the text, albeit, missing some details that correspond to the ignored elements.

One caveat in using VSMs is that their dimensionality is typically high, since it is as large as the vocabulary size of the corpus it is trained on. Latent semantic analysis, discussed next, will address this issue and other related issues. 
Figure 2.3: The figure shows a syntactic dependency parse of a sentence. Tags of syntactic elements are capitalized and their relationships are in small letters. When creating a vector space representation of a linguistic element (e.g., a word), dependencybased VSMs choose what syntactic relationships to capture and disregard any unwanted syntactic relationship or element in the text. This parse is created by TurboParser (Martins et al., 2009, 2010)

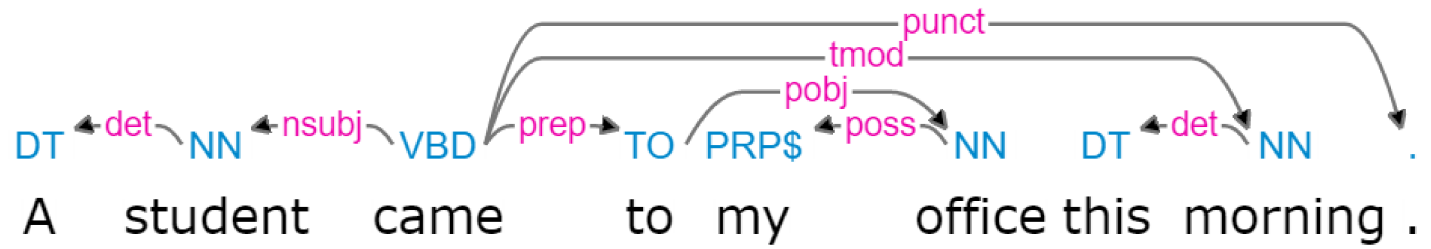

\section{Latent Semantic Analysis}

Latent Semantic Analysis (LSA) is a specific type of VSM that uses Singular Value Decomposition (SVD) to reduce VSMs' dimensionality and further analyze co-occurrences to achieve higher order regularities (Manning and Schütze, 1999; Deerwester et al., 1990). In other words, LSA reveals some higher-order similarities and this results in discovering relationships between words that are hidden from the eyes of basic VSMs. The reason for this is that by reducing the dimensions using SVD, co-occurring terms are mapped onto the same dimensions of the reduced space, and this increases the similarity in representing semantically similar terms (Manning and Schütze, 1999; Deerwester et al., 1990).

We briefly discuss how LSA can be implemented for our purpose. Suppose every term is represented by an $n$-dimensional row vector. If we compile the vectors vertically, a matrix of size $n \times n$ is formed. Let's call this matrix $A$. $A$ is decomposed as follows:

$$
A_{n \times n}=T_{n \times n} S_{n \times n}\left(T_{n \times n}\right)^{T}
$$


We have adopted and modified the equation from $\mathrm{IR}^{13}$ where $A$ contains the frequency of a term per document and, except for $\mathrm{S}$, the matrices are predominantly not square. This is due to the fact that in IR the number of terms and documents considered are often different. In our derived equations, $A$ is a term co-occurrence matrix and hence it is both square and symmetric. For square and symmetric matrices, other approaches such as Eigen analysis ${ }^{14}$ can also be used to reduce dimensionality (Deerwester et al., 1990). We address SVD, since it is the more known and commonly used of the two in sNLP and IR. $S$ is a diagonal matrix that contains singular values of A in descending order (Manning and Schütze, 1999). Then the dimensionality reduction of A is:

$$
B=T_{n \times k} S_{k \times k}
$$

where $k<n$ and $B$ is the dimensionality reduction matrix of $A$.

LSA significantly reduces the computational cost in run time and reveals hidden semantic similarities, although it is noteworthy that the cost in training is still the same. This is mainly due to the large size of the vocabulary. Next, we will address a new VSM algorithm that improves on LSA in terms of performance.

\section{Latent Dirichlet Allocation}

Latent Dirichlet allocation $\left(\mathrm{LDA}^{15}\right)$ is a generative probabilistic model of a collection of documents, where documents are represented as random mixtures over latent topics and each topic is characterized by a distribution over words (Blei et al., 2003). Generative probabilistic modeling regards data as a result of a generative process which

\footnotetext{
${ }^{13}$ Information Retrieval

${ }^{14}$ Eigen analysis decomposes matrices into two matrices of Eigen vectors and Eigen values associated with them

${ }^{15}$ LDA is historically an improvement over probabilistic Latent Semantic Analysis (pLSA) which is a probabilistic graphical model derived from LSA
} 
includes hidden variables. A joint probability distribution is defined over both the observed and hidden random variables. Then this distribution is further analyzed to attain the posterior distribution ${ }^{16}$ of the hidden variables given the observed variables. Words of the documents are the observed variable and the topic structure of the documents comprise the hidden variables. The joint distribution of the LDA is as follows:

$$
p\left(\beta_{1: K}, \theta_{1: D}, z_{1: D}, w_{1: D}\right)=\prod_{i=1}^{K} p\left(\beta_{k}\right) \prod_{d=1}^{D} p\left(\theta_{d}\right)\left(\prod_{n=1}^{N} p\left(z_{d, n} \mid \theta_{d}\right) p\left(w_{d, n} \mid \beta_{1: k}, z_{d, n}\right)\right)
$$

where $\beta_{1: K}$ are the topics, and each $\beta_{k}$ is a distribution over vocabulary; $\theta_{d}$ comprises the topic proportions for the $d$ th document, and $\theta_{d, k}$ is the topic proportion for topic $k$ in document $d ; z_{d}$ holds the topic assignments for document $d$, and $z_{d, n}$ is the topic assignment for the $n$th word in document $d ; w_{d}$ is comprised of the observed words for document $d$, and $w_{d, n}$ is the $n$th word in document $d$ and is an element of our fixed vocabulary. Figure 2.4 below is the graphical model of LDA adopted from Blei (2012).

The posterior of the joint probability mentioned above is:

$$
p\left(\beta_{1: K}, \theta_{1: D}, z_{1: D} \mid w_{1: D}\right)=\frac{p\left(\beta_{1: K}, \theta_{1: D}, z_{1: D}, w_{1: D}\right)}{p\left(w_{1: D}\right)}
$$

where the numerator is the joint distribution of all the random variables. The denominator is the marginal probability of the observations ${ }^{17}$, that is, sum of the joint distribution over every possible instance of the hidden topic structure and it is intractable to compute ${ }^{18}$. To approximate the posterior distribution, two types of algorithms

\footnotetext{
${ }^{16}$ Conditional distribution

${ }^{17}$ Here it translates to the probability of seeing the entire corpus under any topic model

${ }^{18}$ More accurately, this sum is over all possible ways that each observed word of the collection can be assigned to one of the topics and the number of possible topic structures is exponentially large
} 
Figure 2.4: The graphical model for LDA. Nodes denote random variables and shading distinguishes the observed random variable from the hidden ones. Plate $N$ denotes replication over the collection of words in the document, $D$ replication over the collection of documents, and $K$ over the topics (i.e., distributions of the vocabulary). $\alpha$ and $\eta$ are Dirichlet priors hyperparameters and with a uniform base measure they translate into a concentration factor. This figure is adopted from Blei (2012).

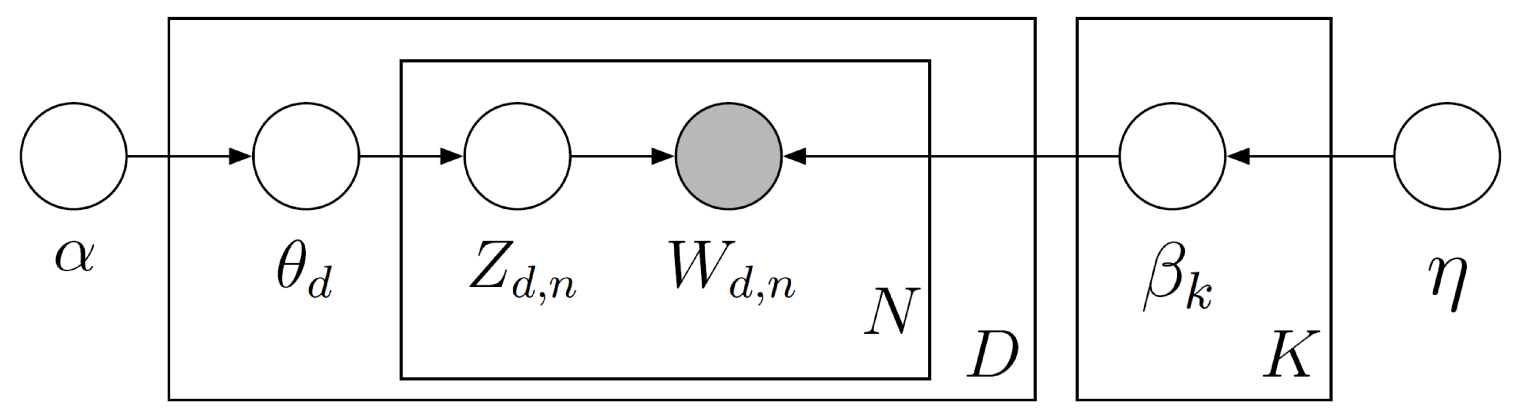

exist: sampling-based algorithms and variational algorithms (Blei, 2012). The former set gather samples from the posterior distribution and try to approximate it with an empirical distribution. Gibbs sampling is the most commonly used algorithm for this purpose. The latter implements a family of distributions over the hidden structure and finds the member closest to the posterior using Kullback-Leibler divergence as a distance measure(Jordan et al., 1999).

LDA, just like LSA, relies on the bag of words assumption which makes it insensitive to word order as opposed to n-gram models and some models based on neural networks discussed in the next section. LDA can be extended to condition the generation of the next word by a topic, on the previous word (Wallach, 2006). Alternatively, LDA can be used in parallel to a Hidden Markov Model (HMM) to preserve word history (Griffiths et al., 2005). Next, we will discuss the last type of statistical models of language called word embeddings some of which inherently preserve word order. 


\subsubsection{Word Embeddings}

Neural networks at first were employed in language modeling by Bengio et al. (2003) to address one of the core issues with n-gram models, that is, their time complexity grows exponentially with $n$. This is also known as the curse of dimensionality throughout machine learning and pattern recognition. This exponential growth in complexity is due to the fact that the occurrence of each word in the sequence is assumed to be fully independent in the count matrices used in n-gram models. This shortcoming has been addressed by class-based n-grams by Brown et al. (1992) where words are assigned to exactly one class based on their usage patterns. Class-based n-grams did improve on ordinary n-gram models; however, they were deemed too rigid due to restricting each word to only one class (Mnih and Hinton, 2009) and they were outperformed by a neural network architecture called Neural Probabilistic Language Model (NPLM) offered by Bengio et al. (2003).

NPLM, as shown in figure 2.5, constructs its language model by decomposing the n-gram model into two main sub-functions $g$ and $C$ :

$$
\hat{P}\left(w_{t}=i \mid w_{1}^{t-1}\right)=g\left(i, C\left(w_{t-1}\right), \ldots, C\left(w_{t-n+1}\right)\right)
$$

where $C$ maps from a word to a real-valued feature vector representation of a fixed size, set as a network parameter, and $g$ maps from an input sequence of vectors of words in the context to a distribution of conditional probabilities over the words in the vocabulary.

Aside from the projection layer, NPLM has a hidden layer with a hyperbolic tangent and is trained by penalized maximum log-likelihood and gradient ascent. It has a softmax output layer that normalizes the output to positive probabilities as 
Figure 2.5: The figure illustrates the Neural Probabilistic Language Model Architecture from Bengio et al. (2003). It has an input layer, a projection layer, a non-linear hidden layer, and a softmax output layer that has the size of the vocabulary where each output node represents the probability of that word in vocabulary being the next word.

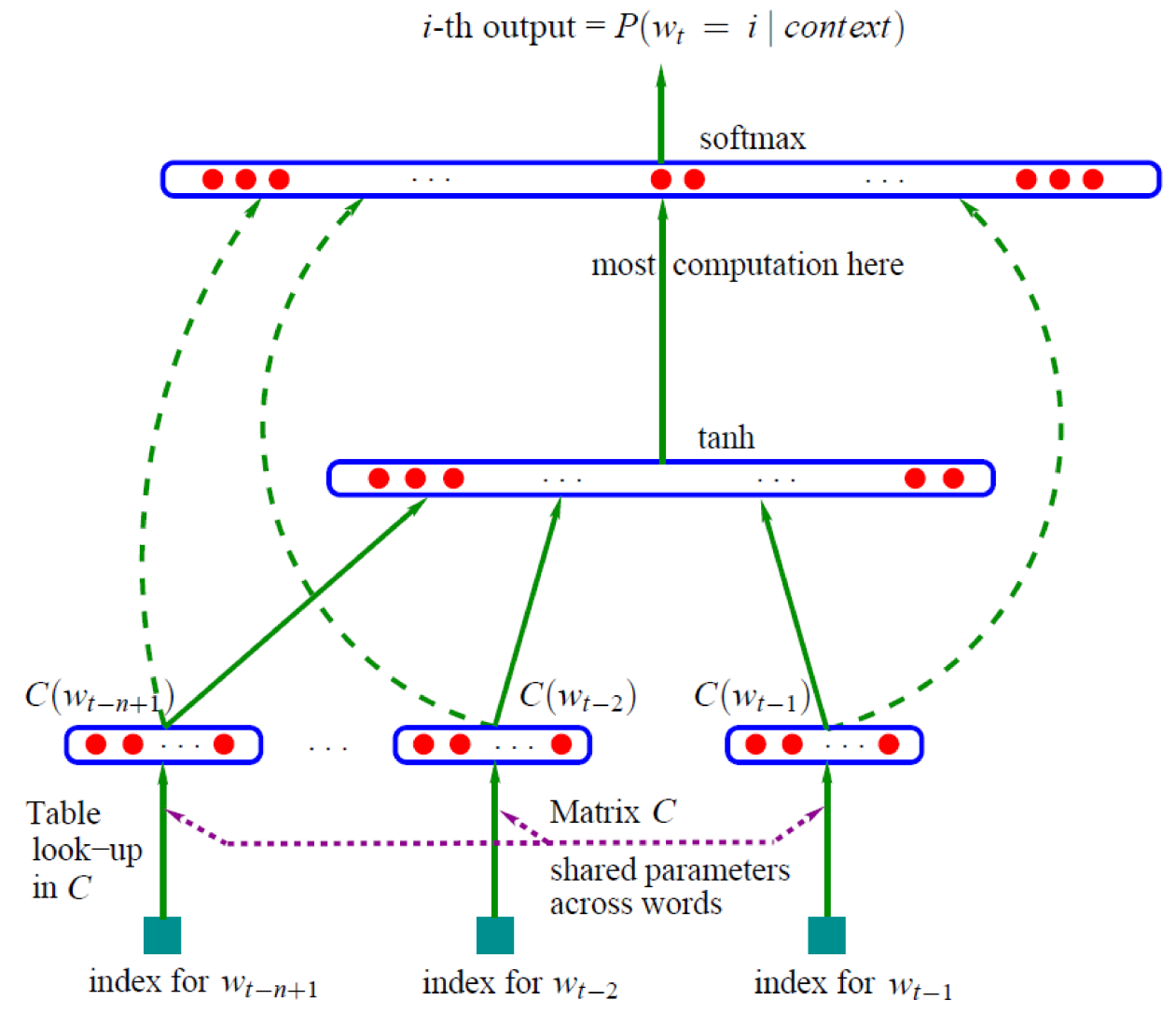

shown below:

$$
\hat{P}\left(w_{t} \mid w_{t-1}, \ldots, w_{t-n+1}\right)=\frac{e^{y_{w_{t}}}}{\sum_{i} e^{y_{i}}}
$$

where $y_{w_{t}}$ is the input sum to the softmax neuron for our $w_{t}$, and $y_{i}$ is the input sum to the softmax neuron for word $i$ in the vocabulary.

The parameters of this model scale only linearly with $n$, the number of grams in the n-gram model, and it improves over the ordinary n-gram models; however, the output 
softmax layer is as big as the vocabulary (to produce probabilities for each word in the vocabulary) which leads to an ultimately much greater computation time than required by n-gram models (Bengio et al., 2003). NLPM, albeit state of the art, is not an efficient approach. To reduce the cost of the search in the output layer, Morin and Bengio (2005) proposed to use a hierarchical decomposition achieved by a balanced binary tree which, in theory, provides an exponential speedup on the order of $\frac{|V|}{\log _{2}|V|}$ (where $|V|$ is the vocabulary size), given that only the probability of one or a few words is desired. But if the probability of all the words are needed, the computational cost is back to $O(|V|)$. In practice, Hierarchical NPLM (HNPLM) showed a remarkable improvement of 2 orders of magnitude over NPLM. In the hierarchical decomposition of HNPLM, each word of the vocabulary is a leaf node in the tree and is represented by a bit vector where the bits mark the sequence of the binary decisions made from the root to reach that leaf. This updates the conditional probability of the next word to:

$$
P\left(v \mid w_{t-1}, \ldots, w_{t-n+1}\right)=\prod_{j=1}^{m} P\left(b_{j}(v) \mid b_{1}(v), \ldots, b_{j-1}(v), w_{t-1}, \ldots, w_{t-n+1}\right)
$$

where $b_{j}(v)$ is the binary decision made at $j$ th node of the tree to reach word $v$ at the leaf, starting from the root.

HNPLM, to achieve its highest efficiency requires the tree to be balanced and made of a sensible distribution of words; that is, words that are more similar to each other should be closer to each other on the tree than those that are less similar. Morin and Bengio (2005) acquire prior knowledge to this distribution using WordNet (Fellbaum, 1998). HNPLM, albeit being exponentially more efficient, does not outperform NPLM. It does, however, outperform every other previous method, including 
ordinary n-gram and class-based n-gram models.

Mnih and Hinton (2009) greatly improved the state of the art for neural language models both in performance in efficiency. They use a simple bilinear model to build word vectors, and, inspired by HNPLM, structure these vectors using a binary tree. In contrast to HPNLM, their approach is fully data-driven, with no expert knowledge required. This method is called Hierarchical Log-Bilinear (HLBL) model and it is built on a previous model called Log-Bilinear (LBL) model from Mnih and Hinton (2007). Given context, the LBL model first predicts the vector of the next word by linearly combining the vectors of the context words:

$$
\hat{r}=\sum_{i=1}^{t-1} C_{i} r_{w_{i}}
$$

where $\hat{r}$ is the estimated feature vector for $w_{t}, C_{i}$ is the weight matrix associated with context position $i$, and $r_{w_{i}}$ is the feature vector pertinent to the context word in position $i$.

Then $\hat{r}$ 's similarity to every vector in the vocabulary is computed by their dot products. These similarities are next exponentiated and normalized to a probability distribution over the next word, as follows:

$$
P\left(w_{t}=v \mid w_{1: t-1}\right)=\frac{\exp \left(\hat{r}^{T} r_{v}+b_{v}\right)}{\sum_{j} \exp \left(\hat{r}^{T} r_{j}+b_{j}\right)}
$$

The biases above account for the context-independent word frequency (Mnih and Hinton, 2007). In contrast to NLPM, the LBL model computes the hidden activities only once per prediction and has no non-linearity in that layer, both of which are factors of its faster performance. The LBL model outperforms the previous models, including NPLM, on a fairly large dataset (Mnih and Hinton, 2007).

The HLBL model has two distinguishing features in contrast to HNPLM. First, 
it uses the LBL model to compute the probabilities at each node. Second, a word has multiple occurrences in the tree, rather than just one. Each sense of the word, therefore, can be uniquely identified by a bit vector $d$ which marks the decisions made at each node, where $d_{i}=1$ means the left branch is traversed. Each nonterminal $^{19}$ node is represented by a feature vector that is used to discriminate the left subtree from the right subtree at that node. As a result, $w_{t}$ is represented by a bit vector, as opposed to the context words that are in the shape of real-valued feature vectors. Nonetheless, as the bit vector of $w_{t}$ is built on the feature vectors of all the non-terminal nodes, together with the binary tree, it still provides a rich and highly informative representation for the model. In the HLBL model, the probability distribution over the next word is determined by the probability of making the binary decisions at the nodes to traverse from the root of the tree to a leaf representing a word in the vocabulary, given the context words. Consequently, the probability of $v^{20}$ being the next word in a context is the probability of the bit vector given that context. Furthermore, the probability of making a decision at each node depends solely on the predicted feature vector that is attained from the context and the feature vector of the node. Accordingly, Mnih and Hinton (2009) formulate the probability of a next word in the HLBL model as the product of probabilities of the binary decisions to reach $v$ :

$$
P\left(w_{t}=v \mid w_{1: t-1}\right)=\prod_{i} P\left(d_{i} \mid q_{i}, w_{1: t-1}\right)
$$

where $d_{i}$ is the $i^{\text {th }}$ value in the bit vector of $v$, and $q_{i}$ is the feature vector for the $i^{\text {th }}$ node on the path to $v$. The probability of each decision picking the right branch is

\footnotetext{
${ }^{19}$ The terminal node being the leaf

${ }^{20}$ Or a sense of $v$
} 
obtained by:

$$
P\left(d_{i}=1 \mid q_{i}, w_{1: t-1}\right)=\sigma\left(\hat{r}^{T} q_{i}+b_{i}\right)
$$

where $\sigma(x)$ is the logistic function, $\hat{r}$ is computed by equation (15) above, and $b_{i}$ is the context independent tendency to pick the left branch at node $i$. Equation (17) can account for the probability of the next word had it had only one instance in the tree, but since every word in the vocabulary has more than one bit vector, equation (17) is updated to equation (19):

$$
P\left(w_{t}=v \mid w_{1: t-1}\right)=\sum_{d \in D(v)} \prod_{i} P\left(d_{i} \mid q_{i}, w_{1: t-1}\right)
$$

where $D(v)$ corresponds to the set of bit vectors ${ }^{21}$ in the tree that are associated with word $v$ in the vocabulary. Having a word in the vocabulary captured by multiple occurrences in the binary tree not only helps address different senses of a word, but also helps leverage multiple hierarchies (not a single one) to capture the manifold nature of its interaction with other words(Mnih and Hinton, 2009).

The HLBL model is faster than HNPLM in prediction time, since it uses a linear, rather than a non-linear, hidden layer to compute the local probabilities. However, the major contributing factor to its speed is that the HLBL model only computes the hidden layer activation functions once per prediction, where HNPLM does it $O(\log V)$ times per prediction. The HLBL model still has its time complexity quadratic in feature vector dimensionality $D$, which makes use of high-dimensional feature vectors computationally costly. Mnih and Hinton (2009) restrict weight matrix $C_{i}$ to be diagonal, which in turn yields a complexity linear in $D$. This updates equation (15) to an element-wise product as follows:

\footnotetext{
${ }^{21}$ In other words different senses or usage patterns of that word
} 
(20) $\hat{r}=\sum_{i=1}^{t-1} C_{i} \circ r_{w_{i}}$

The first step in the HLBL model is to produce the binary tree. To do so, bootstrapping is employed; that is, a random binary word tree is generated, an HBL model is trained on it, then the distributed representation learnt by the HLBL model is used to represent words when constructing the binary tree. To build low-dimensional real-valued vector representation of words, Mnih and Hinton (2009) compute the expectation of the context distribution for each word. This yields a distribution over words where each is represented by a real-valued vector of fixed size. Next, they apply a simple binary clustering on the distribution. Then the same binary clustering is applied on each subset. This procedure is repeated until the current subset contains only 2 words. Words that are difficult to cluster may have multiple codes and the final tree is a merger of multiple run of their algorithm.

The HLBL model is trained by maximizing the log-likelihood with stochastic gradient ascent, with learning rate of $10^{-} 3$ which was reduced to $3 \times 10^{-} 5$ when perplexity on the validation set began to increase. Training stops when the same perplexity begins to increase again. Regulation was applied using a small $L_{2}$ penalty.

Mikolov et al. (2010) employed a different type of architecture $^{22}$, that is, a recurrent neural network architecture to enhance language modeling for automatic speech recognitions. They intuitively called their model Recurrent Neural Network Language Model (RNNLM). Their input layer is the concatenation of a 1-of- $N$ coding of the words in the vocabulary and the context vector (i.e., hidden layer activations) of the previous time step. Therefore the size of the input vector is $|V|+|C O N T E X T(t)|$, where CONTEXT(t) is the context vector at the current time step. The hidden

\footnotetext{
${ }^{22}$ This architecture, called Elman network, was adopted from Elman (1990).
} 
layer activation is initialized to small values ${ }^{23}$. The size of the hidden layer is from 30 to 500 depending on the size of the training data; more units are needed if the corpus and consequently the vocabulary is larger. To train the network, they use the standard backpropagation algorithm with stochastic gradient descent with cross entropy as the error function. The network is initialized by a learning rate of $\alpha=1$ and tested on the validation set after each epoch. If no significant improvement is achieved in predicting the validation set, the learning rate is halved for the new epoch. The training stops if no significant gain in performance is achieved a second time. Their network converges in 10 to 20 epochs. Regularization in their network provided no significant improvement. RNNLM computes the probability of the next word being $w_{i}$ in the vocabulary as follows:

$$
P\left(w_{i}(t+1) \mid w(t), s(t-1)\right)= \begin{cases}\frac{y_{\text {rare }}(t)}{C_{\text {rare }}} & \text { if } w_{i}(t+1) \text { is rare } \\ y_{i}(t) & \text { otherwise }\end{cases}
$$

where $w(t)$ is the word at time $t$ (the current word), $s(t-1)$ is the sigmoid activation vector of the previous word, $C_{\text {rare }}$ is the number of rare words in the vocabulary, $\frac{y_{\text {rare }}(t)}{C_{\text {rare }}}$ provides a uniform probability distribution for the rare words, and $y_{i}(t)$ is the softmax probability output of $w_{i}$ being the next word ${ }^{24}$. Rare words are selected by a frequency threshold from the corpus.

Mikolov et al. (2010) report that RNNLM is faster than NPLM. Being a recurrent network, RNNLM achieves higher performance on much less data as compared to n-

\footnotetext{
${ }^{23}$ Such as 0.1

${ }^{24}$ The next word in RNNLM is shown as $w(t+1)$ and not as $w(t)$ in some of the previous methods as computed at time $t$, since in RNNLM $t$ marks the time stamp in the recurrence of the network and not merely the position of the word.
} 
gram models. They report state of the art language modeling with RNNLM in speech recognition, a $18 \%$ reduction in $\mathrm{WER}^{25}$ for speech recognition on the WSJ task, and a $50 \%$ reduction in perplexity, both reductions were in comparison to a 5 -gram language model with modified Kneser-Ney smoothing ${ }^{26}$. The RNNLM's architecture is shown in figure 2.6 below. It is noteworthy that RNNLM, being a recurrent network, does not require a parameter for the number of history words.

Mikolov et al. (2011) extended the original RNNLM to improve its performance as well as its efficiency. We call this new model eXtended Recurrent Neural Network Language Model (XRNNLM). They showed that Back-Propagation Through Time (BPTT) (Rumelhart et al., 1988; Mozer, 1995) results in a better performance than backpropagation. They also used frequency binning to assign words to different classes so that instead of having to compute the probability distribution over the entire vocabulary, first the probability distribution over the classes is estimated and then the probability distribution over words in each class. Since frequency binning is used each word in the vocabulary can occur only in one class. The output layer is factorized into two parts: word softmax output units and class softmax output units. In backpropagation, the errors of each part are summed up to compute the error derivatives with respect to the hidden layer. Their design speeds up XRNNLM against RNLLM by 15 times in their experiment. They also showed that, in theory, the computational cost can be further reduced by adding a compression layer between the hidden and the output layers, but did not implement such in the above-mentioned study. Four to five time steps in BPTT proved sufficient to cause a significant im-

\footnotetext{
${ }^{25}$ Word Error Rate

${ }^{26}$ Kneser-Ney is one of the most commonly used smoothing methods. Smoothing methods discount the co-occurrence probabilities to eliminate the problem of 0 probabilities and attain a better estimate of n-grams by redeeming sampling error.
} 
Figure 2.6: The figure illustrates the Recurrent Neural Network Language Model Architecture from Mikolov et al. (2010). It has an input layer comprised of a 1-of- $N$ coding and the context layer of the previous time step. It has a logistic hidden layer and a softmax output layer of the vocabulary size.

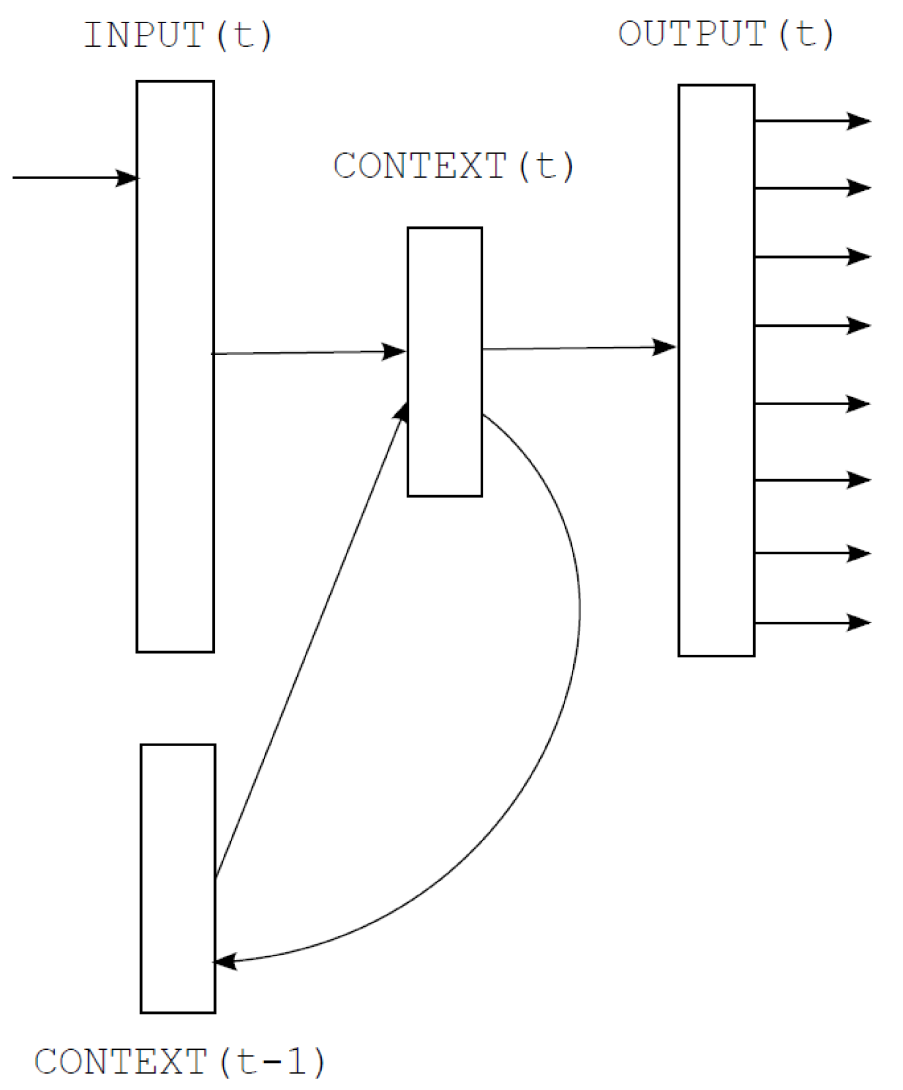

provement in the language modeling task. They illustrated the significant advantage of linear interpolation over averaging different models. These models are to be different in parameters and/or initialization of their random weights to achieve the best performance with linear interpolation. The architecture of XRNNLM is depicted by figure 2.7. Finally, the probability of the next word being $w_{i}$ in XRNNLM given the preceding words (i.e., history) is attained as follows:

$$
P\left(w_{i} \mid \text { history }\right)=P\left(c_{i} \mid s(t)\right) P\left(w_{i} \mid c_{i}, s(t)\right)
$$


where $c_{i}$ is the word class $i$, and the history is retained in the recurrent hidden layer $\mathrm{s}(\mathrm{t})$.

Figure 2.7: The figure illustrates the eXtended Recurrent Neural Network Language Model Architecture from Mikolov et al. (2011). It has an input layer comprised of a 1-of- $N$ coding, a logistic recurrent hidden layer and a factorized softmax output layer.

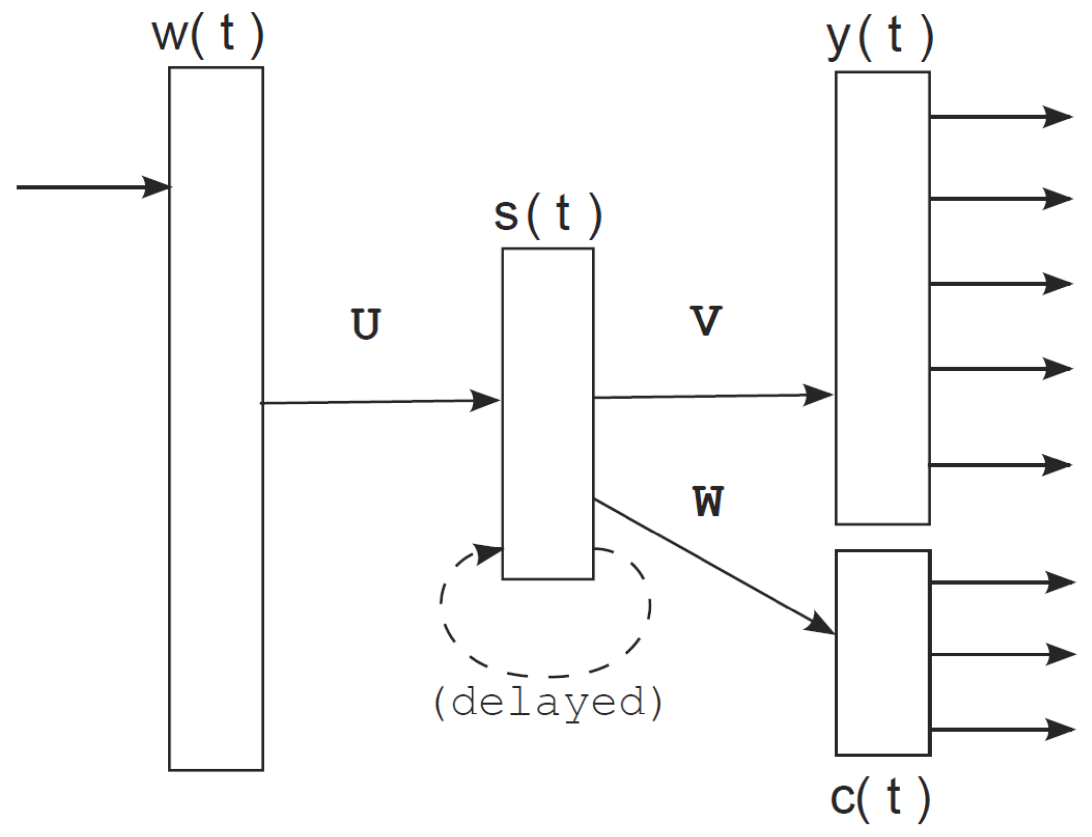

XRNNLM, with a simple design, achieved state of the art performance in language modeling on the Penn Treebank Corpus ${ }^{27}$, outperforming previous more sophisticated algorithms, including Neural Syntactic Language Model (Emami and Jelinek, 2004) which combines syntactic parses with a neural language model similar to NPLM to enhance language modeling.

A momentous landmark in using neural networks in sNLP was molded by Mikolov et al. (2009) training 2 disjoint networks where the first provides a low dimensional vector space for the other; and the second network uses the provided representation

\footnotetext{
${ }^{27}$ https://catalog.ldc.upenn.edu/ldc99t42
} 
to compute n-gram probabilities. Such defied the original recipe proffered by Bengio et al. (2003), presumably in excess of the highest potential n-gram performance. Nevertheless, the idea of using neural networks to bring words into a continuous vector space (Hinton et al., 1986; Rumelhart et al., 1988; Elman, 1990) resurged and they were shown to improve and simplify a variety of NLP tasks (Collobert and Weston, 2008; Turian et al., 2010; Collobert et al., 2011). These continuous low dimensional28 vectors constructed by means of neural networks were popularized as embeddings; and embeddings that represent words in vector space are known as word embeddings, hence the title of the present subsection. Embeddings were shown to outperform LSA and LDA and better preserve linear regularities amongst words. They have been shown to be computationally less costly than LDA (Mikolov et al., 2013f; Zhila et al., 2013).

Mikolov et al. (2013a) decreased the cost of softmax output layer in the previous models with a hierarchical softmax, from $\log _{2}(|V|)$ to $\log _{2}$ (unigram_preplexity $\left.(|V|)\right)$ which translates to a two times speed up for $|V|=1,000,000$. They achieved this by employing a Huffman binary tree instead of an ordinary balanced binary tree. The Huffman tree was employed by every model that they test in their experiments. For training all the models they use mini-batch asynchronous gradient descent with an adaptive learning rate. The models that they experiment on are variants of RNNLM and NPLM as well as two new architecture that they propose, namely, Continuous Bag Of Words (CBOW) and skip-gram models. These new architectures build on the earlier work Mikolov et al. (2009) showing word vectors can be built disjointly from the neural network language model.

\footnotetext{
${ }^{28}$ Often of size $50-1000$
} 
These two new architectures resemble that of NPLM, except for the following differences:

- The non-linear hidden layer is removed.

- Not only the weights of the projection layer are shared but the projection layer itself is shared. As a result, unlike NPLM, there is no explicit order information input into the network.

As can be inferred from the second property above the two new architecture are not designed to predict a next word, but to bring words into the vector space as efficiently as possible. The new architectures are essentially log-linear classifiers.

The CBOW architecture linearly combines a few words in the past, as well as in the future, to correctly classify the correct word in the middle, $w(t)$. This model is called continuous bag of words since, analogous to the bag of words approach, it does not preserve the order of its context words, but unlike bag of words, it uses a continuous vector space for its representation. Mikolov et al. (2013a) acquired the best performance of this model by incorporating 4 words in the past and 4 words in the future. Figure 2.8 illustrates the CBOW architecture.

Continuous skip-gram model, as shown in figure2.9 is the second architecture Mikolov et al. (2013a) proposed. It aims to maximize classification of words surrounding the target word in the middle, $w(t)$, in the same sentence. More weight is assigned to the closer words in random sampling. This is achieved by a parameter, namely $C$, so that for each training case a random number $R$ is generated where $1 \leq R \leq C$, and all the words that fall in distance $R$ from $w(t)$ are sampled as correct labels. In their experiment, they use $C=10$. 
Figure 2.8: The figure illustrates the CBOW architecture from Mikolov et al. (2013a). $w(t)$ in this architecture is a log-linear combination of a few words in the pas and a few in the future.

INPUT PROJECTION OUTPUT

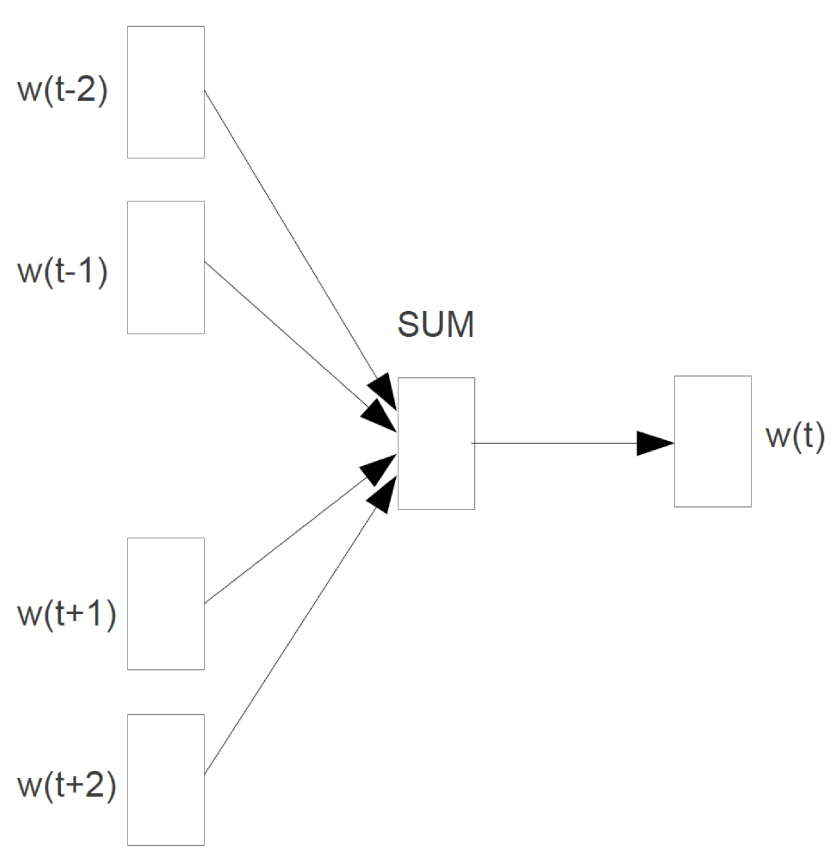

CBOW

Mikolov et al. (2013a) created a semantic-syntactic word relationship test set to assess the quality of all the vectors created by different neural network architectures. For all the architectures in their experiments, they ran 3 epochs with a learning rate of $\alpha=0.025$ where it would linearly diminish to approximately zero by the end of the third epoch.

Mikolov et al. (2013a) find that the CBOW and skip-gram architectures outperform both RNNLM and NPLM, the latter with a much less margin. Skipgram performs best in capturing semantic regularities among pairs of words, despite main- 
Figure 2.9: The figure illustrates the skip-gram architecture from Mikolov et al. (2013a). It is a log-linear classifier that aims to predict the correct $C$ words at each side of the input word $w(t)$, where $C$ is the random sampling parameter.

INPUT PROJECTION OUTPUT

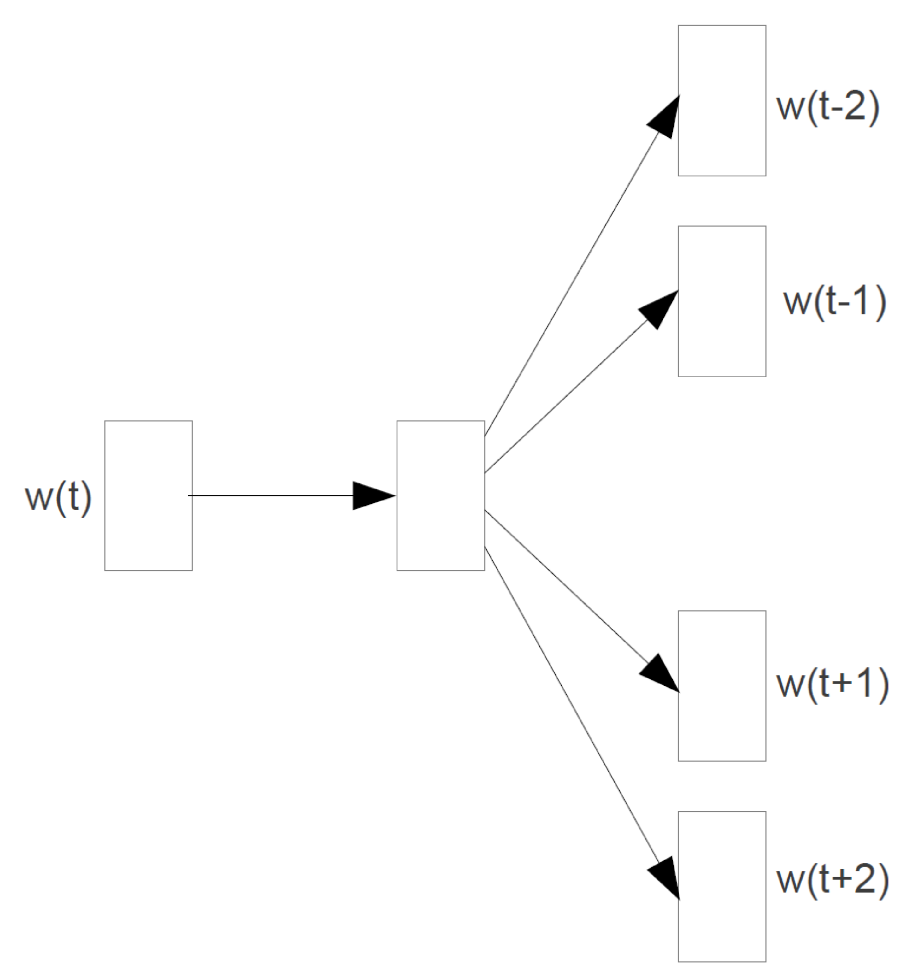

\section{Skip-gram}

taining a high performance in syntax. CBOW, on the other hand, outperforms every model in incorporating syntactic regularities among pairs of words ${ }^{29}$. RNNLM showed the lowest performance. Finally, because of their lower computational complexity, CBOW and skip-gram architectures can be trained on corpora that are several orders of magnitude greater than any other models.

Pennington et al. (2014) offer yet another log-bilinear model which can be consid-

\footnotetext{
${ }^{29}$ Such manifestly does not include predicting the next word in a sequence.
} 
ered a fusion of the global matrix factorization models (e.g., LSA) and what they call local context window models (e.g., CBOW and skip-gram). In matrix factorization methods, the final representation is based on a matrix of the co-occurrence counts of the words in the entire corpus; however, in context window methods, no such global matrix is at all constructed. In fact, no count matrix is ever constructed, since the purpose of the architecture is to directly achieve the optimal vector representation of the words. Context window approaches may ipso facto be called local. Pennington et al. (2014) first scan the corpus for all the words with a context window of size $c$ (typically 10 for each side) and construct their (global) count matrix $X$ then stochastically sample nonzero elements from $X$ feeding the log-bilinear model equipped with AdaGrad Duchi et al. (2011) with an initial learning rate of 0.05. Training on nonzero elements turns the sparsity of the co-occurrence matrix from a demerit into a merit which contrasts with the earlier matrix factorization methods. This new model, therefore, has the traits of both sets of models mentioned above: it has a matrix representing the global co-occurrence counts in the corpus, hence the global statistics of the corpus (the advantage of matrix factorization methods), and it uses a log-bilinear model to manifest each word in a parametrically sized and therefore computationally efficient vector space (the advantage of context window methods).

Pennington et al. (2014) call their model GloVe, standing for Global Vectors for word representation. GloVe is a least squares regression model as follows:

$$
J=\sum_{i, j=1}^{V} f\left(X_{i j}\right)\left(w_{i}^{T} \tilde{w}_{j}+b_{i}+\tilde{b}_{j}-\log X_{i j}\right)^{2}
$$

where $f\left(X_{i j}\right)$ is the weighting function that takes the co-occurrence count of the words $i$ and $j$ as input and outputs a weight, $w_{i}$ and $\tilde{w}_{j}$ are the word vectors, the 
tilde indicates they each come from a distinct vector space, and finally $b_{i}$ and $\tilde{b}_{j}$ are their corresponding biases.

If $X$ is symmetric the only difference between $w$ and $\tilde{w}$ is in their random initialization. The sum of both these types of vectors is used as the final representation for each vocabulary item in GloVe. The previous context window models grow linearly in the size of the corpus $\mathrm{C}$, that is $O|C|$, GloVe on the other hand scales slightly better than the previous context window models $O|C|^{0.8}$. To evaluate the quality of GloVe's vectors, they were appraised against the standard variation as well as recent and more efficient variations of LSA with SVD's of $\sqrt{X_{\text {trunc }}}$ and $\log \left(1+X_{\text {trunc }}\right)$, where $X_{\text {trunc }}$ retains the co-occurrence counts of each word with only the top 10,000 most frequent words ${ }^{30}$. GloVe was also assessed in comparison with the recent context window models (including but not limited to CBOW and skip-gram). It outperformed the competitors on the word analogy task and word similarity task intrinsically ${ }^{31}$ and named entity recognition with Conditional Random Field (CRF), extrinsically ${ }^{32}$.

\subsection{Summary}

Section 2.1 discussed the various methods in the task of automatic terminology extraction and their evolution. Section 2.2 introduced transfer learning and one way it can be exploited in sNLP tasks. Transfer learning is the basis of the methods offered in this dissertation. Section 2.3 introduced domain adaptation and discussed the previous methods that applied it to the task of bilingual word mapping which

\footnotetext{
${ }^{30}$ Rare words are argued to be less informative (Pennington et al., 2014)

${ }^{31}$ That is, the performance on the analogy task is considered a direct reflection of the quality of the vectors.

${ }^{32}$ That is, the vectors were used part of the algorithm and the performance on the task is indirectly reflective of the quality of the used vectors.
} 
is different from ATE but does have some similarities. The present work is the first to apply domain adaptation to ATE. Section 2.4 was an effort to provide a concise but thorough account of the available methods to transform words from a symbolic representation to a rich vector space; not only the advantages of each, but also their bottlenecks were discussed, leading to the more advanced methods. In the experiments put forward by this dissertation, we leverage the efficiency and accuracy of the three latest and best performing methods discussed in this section, namely, CBOW, skip-gram, and GloVe. This section plays a fundamental role to support our choice of representation as well as to provide technical background for the discussions and details in the next chapters. 


\section{Chapter 3}

\section{Automatic Terminology Extraction}

\section{Evaluation: A Gold Standard for}

\section{the Mathematics Education}

\section{Domain}

The quality of the terms extracted by Automatic Terminology Extraction (ATE) algorithms is not always high or even known in advance for all domains. Therefore, an important consideration in terminology extraction is to assess the quality of the extracted terms for a selected domain. This chapter will study the correctness of terms extracted by three term-extraction tools. The annotation is facilitated by a term annotation tool which makes available domain-specific dictionary, a set of filters, and an annotation memory, and allows for post-hoc evaluation. We present a study in which two human judges used the developed tool for term annotation. Their 
annotations were then analyzed to determine the efficiency of term extraction tools by measures of precision, recall, and F-score, and to calculate the inter-annotator agreement rate. Aside from determining the performance of the current methods in ATE, this chapter provides us with a dataset whereby new methods can be evaluated and benchmarked for the mathematics domain.

\subsection{Introduction}

In the field of Natural Language Processing (NLP), Automatic Terminology Extraction (ATE) is a subtask of information extraction. Its goal is to automatically extract relevant terms from a given corpus. The present study is part of an endeavor towards finding available efficient terminology extraction software tools for extracting subjectspecific terminology from academic textbooks. Hence, an immediate concern was to assess the extraction performance of these tools. We use a tool called Term Evaluator to facilitate the annotation task and the term extraction evaluation.

A significant component of any academic and educational subject is its terminology. Knowledge of the terminology of a field enables students to engage with their discipline more effectively by enhancing their ability to understand the related academic texts and lectures, and allowing them to use the subject-specific terminology in their discussions, presentations and assignments. Therefore, generating lists of terminology specific to various fields of study is a significant endeavor. However, these lists have often been generated manually or through corpus-based studies, which are time consuming, labor-intensive, and prone to human error. Therefore, an automatic terminology extraction procedure can facilitate this work to a great extent.

Terminology extraction has many direct applications in NLP, such as information 
retrieval, machine translation, parsing sublanguages, question-answering, and ontology construction. It underwent a rapid rise and growth throughout the nineties, and computational terminology diversified into many subtasks (Nazarenko and Zargayouna, 2009), including relation extraction, variation calculus, and term normalization. We recognize the subtask decomposition protocol (see section 3.2 for details) proposed by Nazarenko and Zargayouna (2009), but in this study we focus only on evaluating the terminology extraction subtask.

Terminology extraction has traditionally been accomplished by using three different methods, namely, linguistic, statistical, and hybrid, and according to two major criteria: termhood and unithood (Castellví et al., 2001; Chung, 2003). These ATE methods have been applied to both mono-lingual and multilingual corpora (Ljubešić et al., 2012). Termhood is the degree of a linguistic unit being related to a domainspecific concept, and unithood is defined as the degree of stability of the syntagmatic combination (Kageura and Umino, 1996).

In the next section, we discuss previous work related to ATE evaluation. In section 3.3, we introduce the tools under evaluation and provide details on their extraction methods. Section 3.5 briefly outlines our corpus and how it was compiled. Section 3.6 introduces the term evaluator tool employed, some of its main functionalities, and its user interface. Section 3.7 provides the details of the annotation process. The analysis and the results are presented in section 3.8. Section 3.9 provides a summary of the presented work. 


\subsection{Related Work}

CoRReCT was one of the first to present a dataset and protocol for term recognition in corpora. The task consisted of taking a corpus and terminology as inputs and indexing the corpus with the terms in their standard and variant forms (Enguehard, 2003).

CESART offered a complete evaluation project (Hadi et al., 2006), involving 3 tasks: term extraction, controlled indexing, and relation extraction, but only the first task led to an evaluation. CESART proposed a protocol for term extraction. A gold standard and a corresponding acquisition corpus were developed for a specific domain.

Loginova et al. (2012) manually created Reference Term Lists (RTLs) to serve as gold standards for ATE evaluation of monolingual term candidate lists automatically extracted from Spanish texts in the wind energy domain. Their domain-specific text was automatically obtained by a web crawler. Their RTLs included both singleword and multi-word terms, as well as their graphical, morphological, and syntactic variants. They also accounted for paradigmatic variants of multi-word terms. To create the RTLs, they performed tokenization, part-of-speech (POS) tagging, and lemmatization on the crawled text. Terms were extracted using POS patterns. They also used "weirdness ratio" as a filter on the extracted terms. Creating gold standard RTLs has its own challenges, especially with large corpora. If it is done entirely manually, it is time-consuming; if some NLP systems are used (e.g., lemmatizers and POS taggers), their errors are escalated (Loginova et al., 2012) and some patterns may be missed. Moreover, ATE tools may return some correct terms that have not been detected by the search procedure adopted to create the RTLs, and as a result a correct term may be dismissed. 
Two types of error usually occur in term extraction (Love, 2000): Silence $^{1}$ is the error where the system fails to extract terminological units in the text. Noise ${ }^{2}$ is the error where the system extracts a non-terminological unit. These two errors mirror recall and precision, respectively, that are often used for measuring the performance of different methods (Frantzi et al., 2000; Fedorenko et al., 2014). To compute the performance of the tools under evaluation, we adopted the standard set of scores: precision, recall, and F-score.

\subsection{Term Extraction Methods and Tools}

Term extraction methods usually extract candidate terms and rank them in order to keep only those that can be considered domain-specific terms (Vasiljevs et al., 2014). Tokenization, part-of-speech tagging and lemmatization are often employed in term extraction algorithms. To extract terms, statistical methods compare the frequency of candidate terms in the target corpus against a general reference corpus (Fedorenko et al., 2014). Linguistic methods use linguistic patterns to detect and extract terminology.

After an initial evaluation of a number of ATE tools, we chose to further evaluate the capability of four promising ones for our purposes, namely, AntConc, Topia, TermoStat, and Sketch Engine, each of which is discussed below. We chose these tools because they were publicly accessible and because they employ different term extraction methods. However, since Sketch Engine extracted a limited number of terms (see below for further details), we did not evaluate its output. Lack of availability or

\footnotetext{
${ }^{1}$ Corresponds to false negatives in the confusion matrix for an information extraction task.

${ }^{2}$ Corresponds to false positives in the confusion matrix for an information extraction task.
} 
limited input method, size, and format were some of the disadvantages of the other tools that we looked at and excluded from the study.

\subsubsection{AntConc}

AntConc (Anthony, 2012) is the first tool we examined for our term extraction. This tool is widely used in linguistics and corpus linguistics. AntConc has a dedicated keyword extraction module, but it only extracts keywords (composed of one word). Thus, we could not use this functionality as we were interested in terms ${ }^{3}$ composed of one or more words. We used AntConc to extract single-word and multi-word terms by using the "Word List" and "N-Grams" modules respectively, which list the words and multi-word expressions sorted by the frequency of occurrence in the corpus. We designate this approach implemented by AntConc as our evaluation baseline which reflects the role of pure frequency for term extraction in this experiment.

\subsubsection{Topia}

Topia is a hybrid term extraction tool, and uses simple linguistic and statistical procedures to extract terms. We performed the term extraction task by Topia ${ }^{4}$ using the topia.termextract 1.1.0 library. Topia uses a naive POS tagger which operates after tokenization; for each word, its most frequent tag is assigned as its POS tag. Then, some simple rules are applied to extract terms (e.g., excluding terms with frequency 3 and below). We modified the implementation of the Topia library by adding some checking statements (i.e., a filter) to change all the terms which contained

\footnotetext{
${ }^{3}$ There is a further distinction between keywords and terms. Keywords are usually extracted from one text in order to show what the text is about; they are not necessarily domain-specific. Terms are domain-specific and are usually extracted from large corpora of the domain.

${ }^{4}$ Available at: https://pypi.python.org/pypi/topia.termextract/.
} 
numbers and special Unicode characters. We replaced these characters with white space and removed all the terms that included only one or two letters. Topia extracts multiword terms as well as single-word terms, and outputs a single list of terms; therefore, we implemented a script to split the list into four lists, corresponding to one of our four term categories, namely 1-word, 2-word, 3-word, and 4-word terms.

\subsubsection{TermoStat}

TermoStat is a non-commercial web-based terminology extraction software program, and takes a single corpus file as input. It is also a hybrid term extraction system that uses both linguistic clues and statistical techniques to extract candidate terms. TermoStat extracts single-word terms, as well as multi-word terms. For extracting multi-word terms, it restricts the lexical items that can appear inside candidate terms. If a candidate term is included in a longer candidate term and never occurs independently, it is a term fragment and is consequently excluded from the candidate-term list (Drouin, 2003).

TermoStat computes the specificity of a (multi)word in a corpus with reference to a general corpus (described below) by means of a statistical test developed to target highly specific technical terms ${ }^{5}$. There are three outcomes: SP0, SP+, and SP-, meaning the observed frequency in the corpus is consistent, significantly higher, or significantly lower, respectively, with regard to the reference corpus. $\mathrm{SP}+$ constructs a corpus-specific vocabulary which Drouin (2003) calls Specialized Lexical Pivots or SLPs for short.

The reference corpus contains approximately 8 million tokens, corresponding to

\footnotetext{
${ }^{5}$ See Drouin (2003) for more details on the statistical test.
} 
approximately 465,000 different word forms. It is a non-technical corpus, half of which comes from newspaper articles on a variety of subjects from the Montreal daily newspaper 'The Gazette' published between March 1989 and May 1989. The other half of the corpus comes from the British National Corpus (BNC).

TermoStat uses Brill's Tagger to POS-tag its corpora. Any noun in SLPs may be considered a headword. It locates all the headwords within the corpus, and starts the term extraction process from right to left. TermoStat uses both the POS of the words, as well as the results of its statistical process and some part of the formatting of the corpus to determine boundaries which may delimit candidate terms. Only terms in SLPs may be qualified as boundaries. The linguistic structure of the candidate terms retrieved by TermoStat is as follows:

$$
(\mathrm{A} \mid \mathrm{N}) ?(\mathrm{~A} \mid \mathrm{N}) ?(\mathrm{~A} \mid \mathrm{N}) ?(\mathrm{~A} \mid \mathrm{N}) ?(\mathrm{~A} \mid \mathrm{N}) ? \mathrm{~N}^{6}
$$

All the elements in the formal language must exist in SLPs as dictated by TermoStat's formal grammar, and as observed by the above regular expression; the length of six words for a candidate term is imposed. Our corpus was fed to TermoStat for term extraction. We updated our script to split the terms extracted by TermoStat into the following categories: 1-word, 2-word, 3-word, and 4-word terms.

\subsection{Sketch Engine}

This is a tool that we investigated but did not experiment with as it proved not suitable for our purposes. Sketch Engine ${ }^{7}$ (Kilgarriff et al., 2004, 2014) uses a lemmatizer,

\footnotetext{
${ }^{6}$ ' $\mathrm{A}$ ' is an adjective, ' $\mathrm{N}$ ' is a noun, ' $(\mathrm{A}-\mathrm{N})$ ' is a noun or an adjective, ? represents zero or one occurrence of the element immediately preceding, all the elements belong to the SLP set.

${ }^{7}$ Sketch Engine is accessible via http://www.sketchengine.eu
} 
TreeTagger $^{8}$ (Schmid, 1999) for POS tagging, and the following statistical method for computing the specificity of the terms ${ }^{9}$ :

$$
\text { specificity score }=\frac{f p m_{\text {focus }}+n}{f p m_{\text {reference }}+n}
$$

where $\mathrm{fpm}_{\text {focus }}$ is normalized (per million) frequency of word in focus corpus, fpm $_{\text {reference }}$ is normalized (per million) frequency of word in reference corpus, and $n^{10}$ is a simple smoothing parameter to avoid division by zero (by default $n=1$ ).

For a quick experiment with this tool, we used the default value for n. As for reference corpora, we used 3 corpora in 3 different settings and the outcome was almost identical. We used the Brown corpus (small size, approximately 1 million tokens), the British National Corpus (BNC, medium size, approximately 100 million words), and the Web corpus English TenTen 2012 (EnTenTen, large size, approximately 13 billion tokens).

Sketch Engine extracted a total of only 36 multiword terms (excluding singleword terms) and this size is not comparable to the outputs of the other 3 tools (i.e., TermoStat: 1109, Topia: 724, and AntConc: 707). This minimalism may be due to precision/recall trade off enforced by its algorithm for practical purposes. We did not further evaluate Sketch Engine's output for the comparability reason stated above.

\footnotetext{
${ }^{8}$ Sketch Engine uses the grammatical relations (extracted by its engine) for multi-word term extraction.

${ }^{9}$ More about Sketch Engine statistics may be found at: https://www.sketchengine.co.uk/documentation/attachment/wiki/SkE/DocsIndex/skestat.pdf?format $=$ raw

${ }^{10}$ We tested various values for this parameter but they had no significant effect on the number of extracted terms.
} 


\subsection{Corpus}

The corpus that we used for evaluating the three term extraction tools comprised of five English high school mathematics textbooks: Small et al. (2005); Small et al. (2007a); Small et al. (2007b); Crippin et al.; Kirkpatrick et al. (2007). We converted the PDF files of the books into plain text, and then concatenated all the text files into one corpus consisting of 1,127,987 tokens.

\subsection{Term Evaluator}

Term Evaluator is a tool developed and made publicly ${ }^{11}$ available to facilitate the procedure of comparing the performance of the term extraction tools. It provides a user-friendly interface that speeds up the annotation process. It enables a semiautomatic post-hoc evaluation as described below.

The extracted terms are fed into Term Evaluator for annotation. Term Evaluator allows a user to start a fresh evaluation, resume a previous one, load a saved evaluation, and compare two or more evaluations. Users can also configure the term filters and load term lists. Term Evaluator hosts a built in technical dictionary comprised of three merged online mathematics dictionaries ${ }^{12}$ is built in the tool. A secondary

\footnotetext{
${ }^{11}$ TermEvaluator can be downloaded from https://sourceforge.net/projects/termevaluator/

${ }^{12}$ The dictionary belongs to the mathematics domain and was retrieved and compiled from the following three sources:

1. Illustrated Mathematics Dictionary. (n.d.). $\quad$ Retrieved 2013. (http://www.mathsisfun.com/definitions/index.html)

2. Mathwords. (n.d.). Retrieved 2013. (http://www.mathwords.com/)

3. Math Dictionary. (n.d.). Retrieved 2013. (http://www.mathematicsdictionary.com/mathvocabulary.htm)
} 
list of terms on whose correctness the annotators had already agreed (from previous annotation experiments, if any) may also be uploaded. Users can, however, choose not to use the built-in math dictionary, replace it with another dictionary for the same domain, or a dictionary for another domain. If required, Term Evaluator can perform two automatic operations (filtering) on any input: a) It filters out every term from the list that is a stop word (omission of terms), and b) It drops the stop word portion if a term starts or ends with a stop word (change of terms). Figure 3.1 presents the evaluation interface where annotators can assess the extracted terms. They have access to the rank, frequency, and the termhood score of the term at hand and they can mark the category of each term as Yes (technical ${ }^{13}$ ), Non-Technical, No (non- term $^{14}$ ), and Not Sure.

The annotators have the option to view only the items not evaluated before or only those in conflict with other annotators' evaluations. They can also save the evaluation and return to it at a later time. In addition, a list view is available to show all the terms including those annotated or to be annotated (see figure 3.2). Correct terms may be exported at any time during annotation.

Term Evaluator can compare different evaluations, show the number of agreements/disagreements, intersection of annotation decisions, inter-annotator agreement rate, and a few more statistics and comparison details (see figure 3.3). Inter-annotator agreement rate is computed as shown in the equation below.

$$
a g r=\frac{N_{a}}{\left(N_{a}+N_{d}\right)}
$$

where $N_{a}$ is the number of instances the annotators agreed on, and $N_{d}$ is the number

\footnotetext{
${ }^{13}$ Also referred to as "correct" in our tool

${ }^{14}$ Also referred to as "wrong term" in our tool
} 
of instances they disagreed on.

Figure 3.1: The figure shows the annotation window from Term Evaluator.

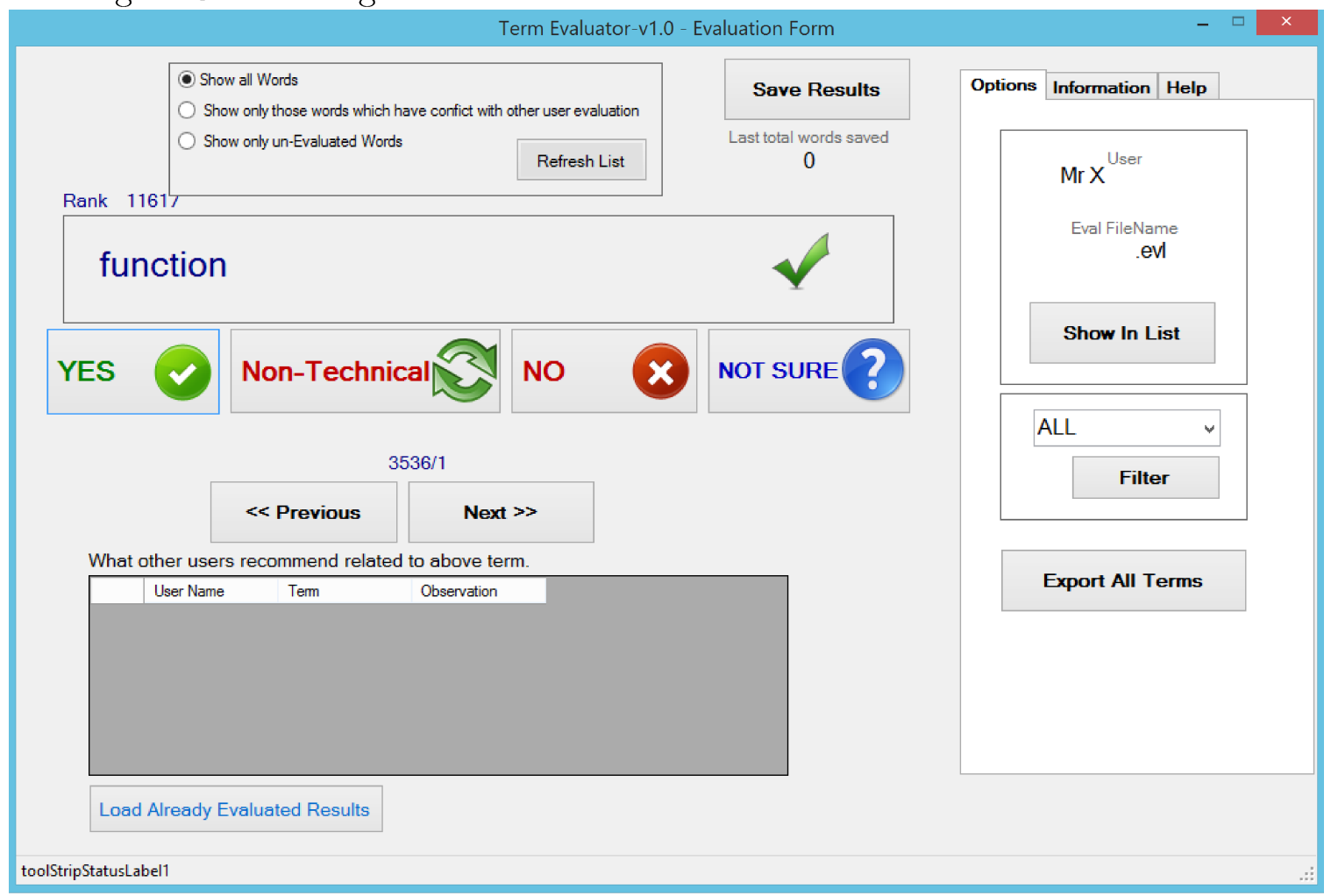

In figure 3.3 the term "correct" refers to technical terms and "wrong terms" refer to non-terms.

\subsection{The Annotation Process}

After term extraction was performed by the term extraction tools, a cut-off value was applied to each of the four word categories (i.e., 1-word, 2-word, 3-word, and 4 -word). The outputs that were already below the threshold, remained intact. The cut-off value was set at $500^{15}$ (if the list of candidate terms was shorter than the

\footnotetext{
${ }^{15}$ In a later experiments, annotators evaluated all the terms extracted by TermoStat (4011 terms).
} 
Figure 3.2: The figure shows the list view annotation window from Term Evaluator.

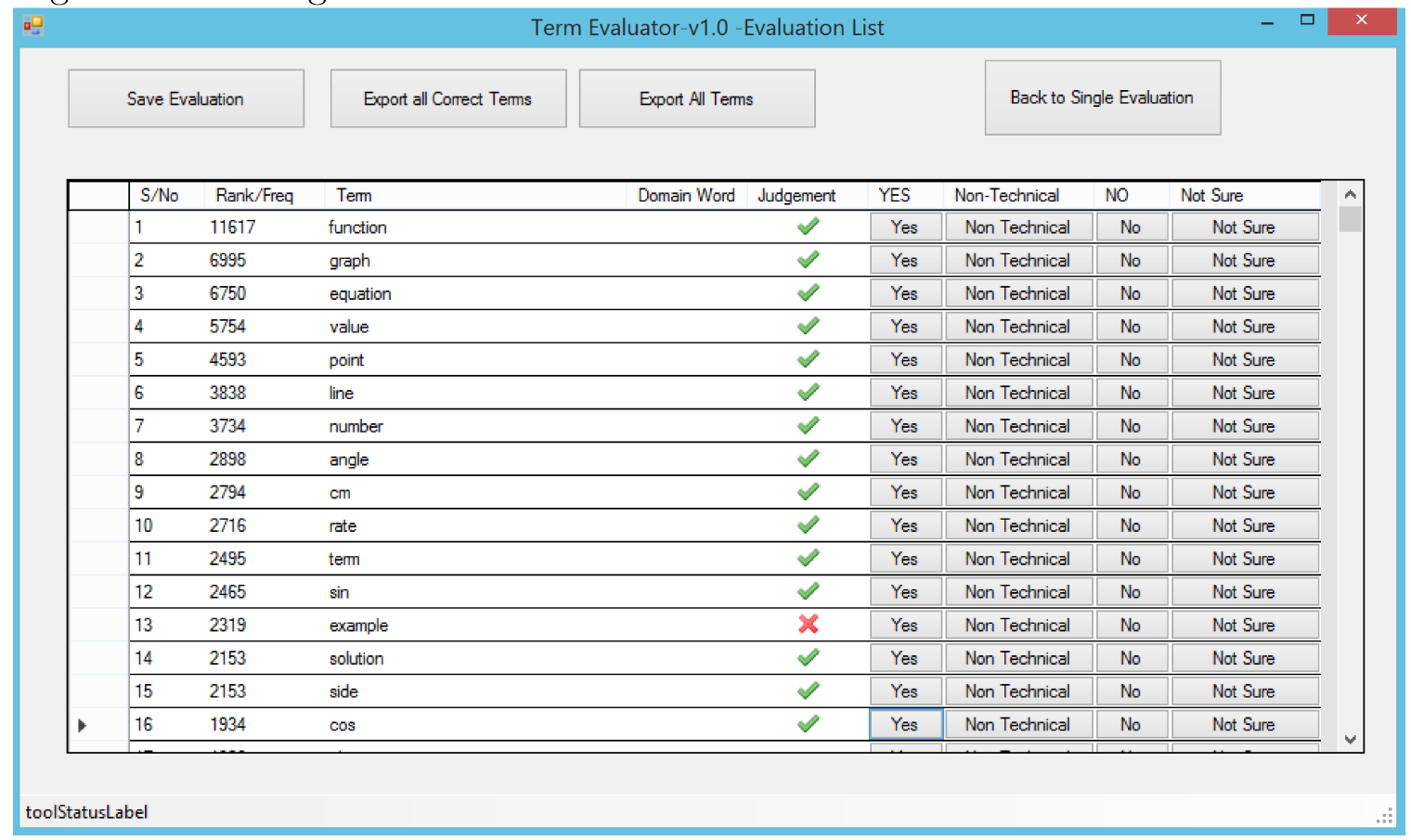

cut-off value, the whole list was retained). For each of the three tools, 4 files were submitted to the annotators corresponding to one of the four term categories.

Two human annotators (one male and one female) judged the terms extracted by the term extraction tools. They were PhD students in education at the time. The annotators were instructed to use the Term Evaluator software to judge the terms using one of the following four options that are provided as buttons in figure 3.1 and figure 3.2: A) YES [technical term], B) Non-Technical [generic English term], C) NO (non-term, D) Not Sure. The definition of these categories were provided to them.

The definitions of these options were provided and the annotators were asked to use their background knowledge of mathematics as the primary source of their judgment. In case of confusion, they could consult a Mathematics dictionary of their choice. 
Figure 3.3: The figure shows the comparison window from Term Evaluator that allows comparing two or more annotators in regards to their annotations.

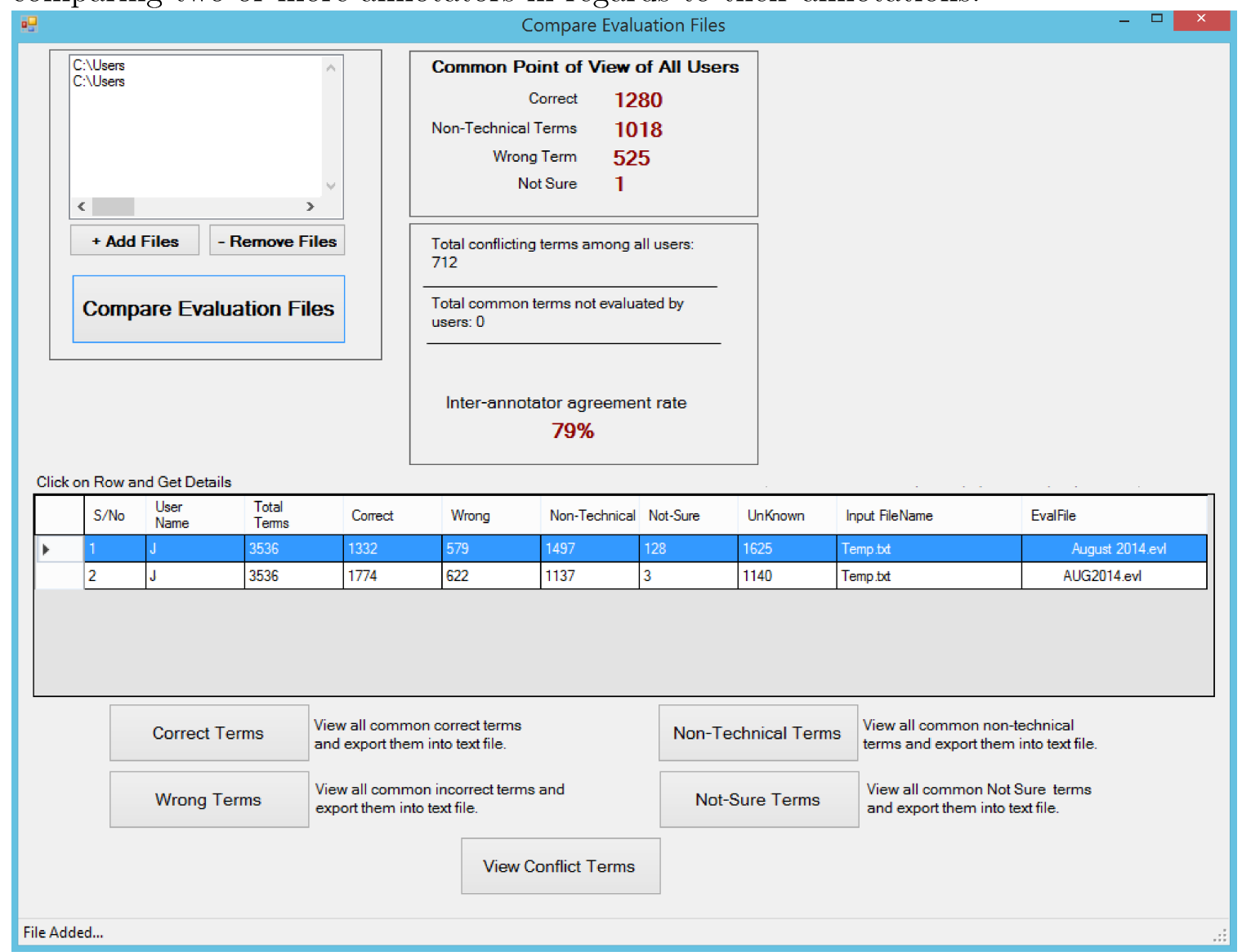

\subsection{Results and Analysis}

We computed precision, relative recall and balanced F-score for each tool. Relative recall is computed against the union of all the predicted correct terms among the term extraction tools, with two categories: correct $^{16}$ and incorrect ${ }^{17}$. The performance of the 3 tools is compared in figures 3.4, 3.5, 3.6, and 3.7 below and in table 3.1. Figure 3.4 shows the performance of the tools for extracting terms that contain only one

\footnotetext{
${ }^{16}$ Technical terms

${ }^{17}$ Non-technical, non-term, and not sure
} 
word. Topia, with the added filter (see section 3.3.2 for details) outperformed the other tools for single-word terms, and had the highest precision, recall and F-score. This is interesting, considering that Topia does not use any sophisticated algorithm. In terms of precision, AntConc comes second and TermoStat last and regarding recall, TermoStat performs better than AntConc. This is also interesting. As mentioned earlier (section 3.3.1), AntConc extracts terms based on basic frequency. The fact that TermoStat has a better recall than AntConc (53\% vs. $51 \%$ respectively) can be an indication that bare frequency may not be sufficient to extract correct terms in a technical corpus. On the other hand, the fact that AntConc achieved a better precision than TermoStat (41\% vs $37 \%$ respectively) confirms the intuition that single words that are frequent in a technical corpus have a high chance of being identified as a term specific to that corpus.

Figure 3.4: Comparison of the performance of the term extraction tools in extracting single-word terms

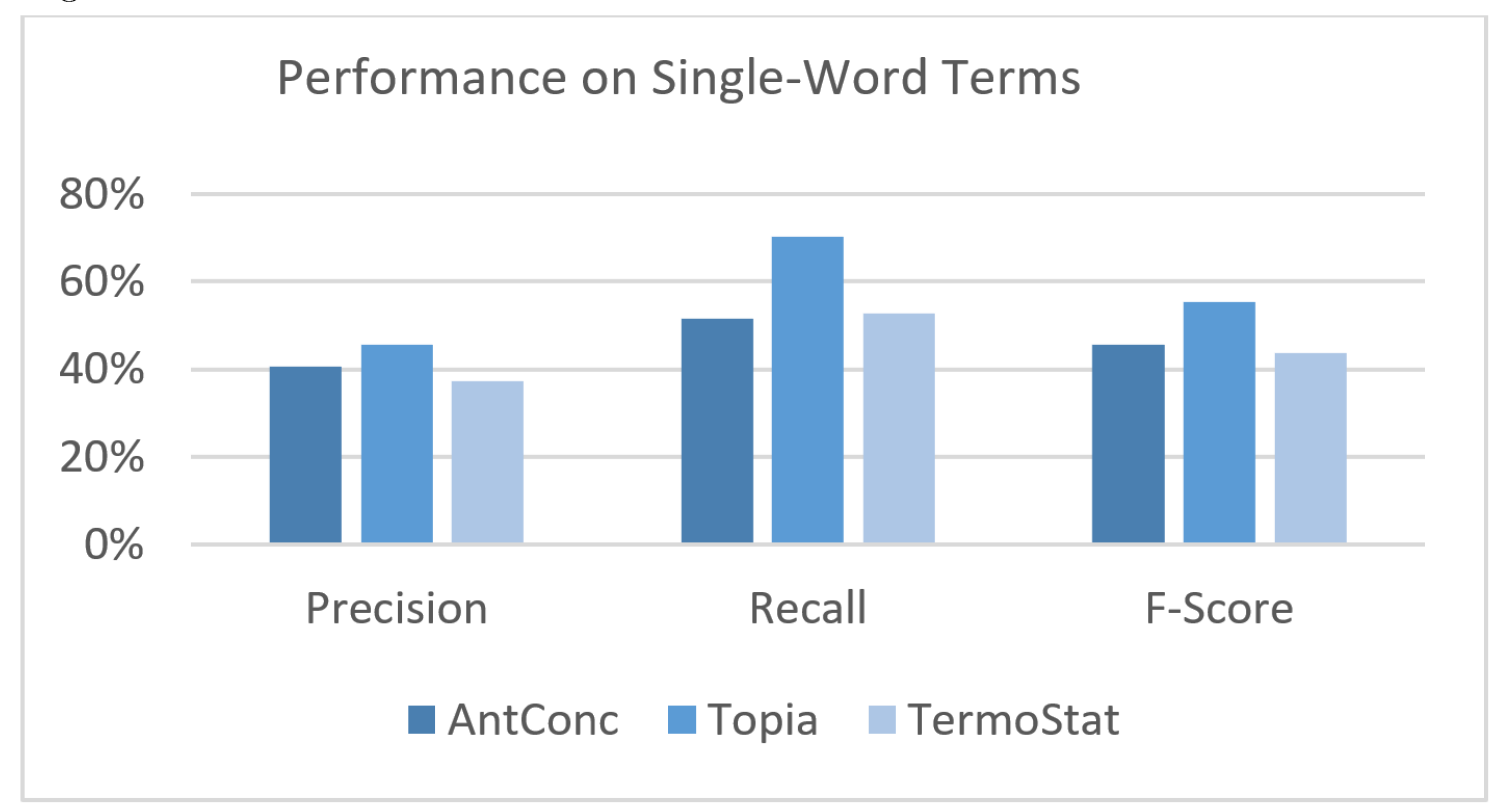


Figure 3.5 presents the performance of the tools in extracting two-word terms from the math corpus. There are a few points that deserve further attention. TermoStat shows a leap from single-word (F-score of 44\%) to two-word term extraction (F-score of $67 \%$ ). Its precision has improved with 31 percentage points and its recall with 13 percentage points. This makes TermoStat the highest performing tool for the two-word term category. This high performance manifests an adequate account of termhood and unithood. Topia is keeping up although it suffers from a simplistic POS tagger as compared to TermoStat that features the well-known and well-performing Brill's tagger (Brill, 1992). POS tagging comes more into play as the number of terms in a multi-word expression increases. The other factor worth mentioning is the competitive precision of AntConc (albeit its low recall scores) that postulates frequent n-grams have a high chance of being terms. It is possible that AntConc's high performance on single-word terms is due to chance (i.e., unigrams); after all, frequent words are probable to be terms.

Figure 3.6 depicts the performance of the tools in extracting three-word terms. What appears striking at first glance is Topia's extreme reduction in performance. TermoStat consistently has the highest precision and recall.

Figure 3.7 presents the performance of the tools in extracting four-word terms. TermoStat still has the lead in both precision and recall. AntConc still has a higher precision than recall and keeps following the same trend as in the two-word and three-word categories. Therefore, except for the single-word terms, n-gram raw frequency does not seem to compete with a proper term extraction algorithm. Topia's performance stays poor for the four-word category ( $11 \%$ precision and $6 \%$ recall).

We computed the overall performance of each tool (table 3.1). TermoStat achieved 
Figure 3.5: Comparison of the performance of the term extraction tools in extracting two-word terms

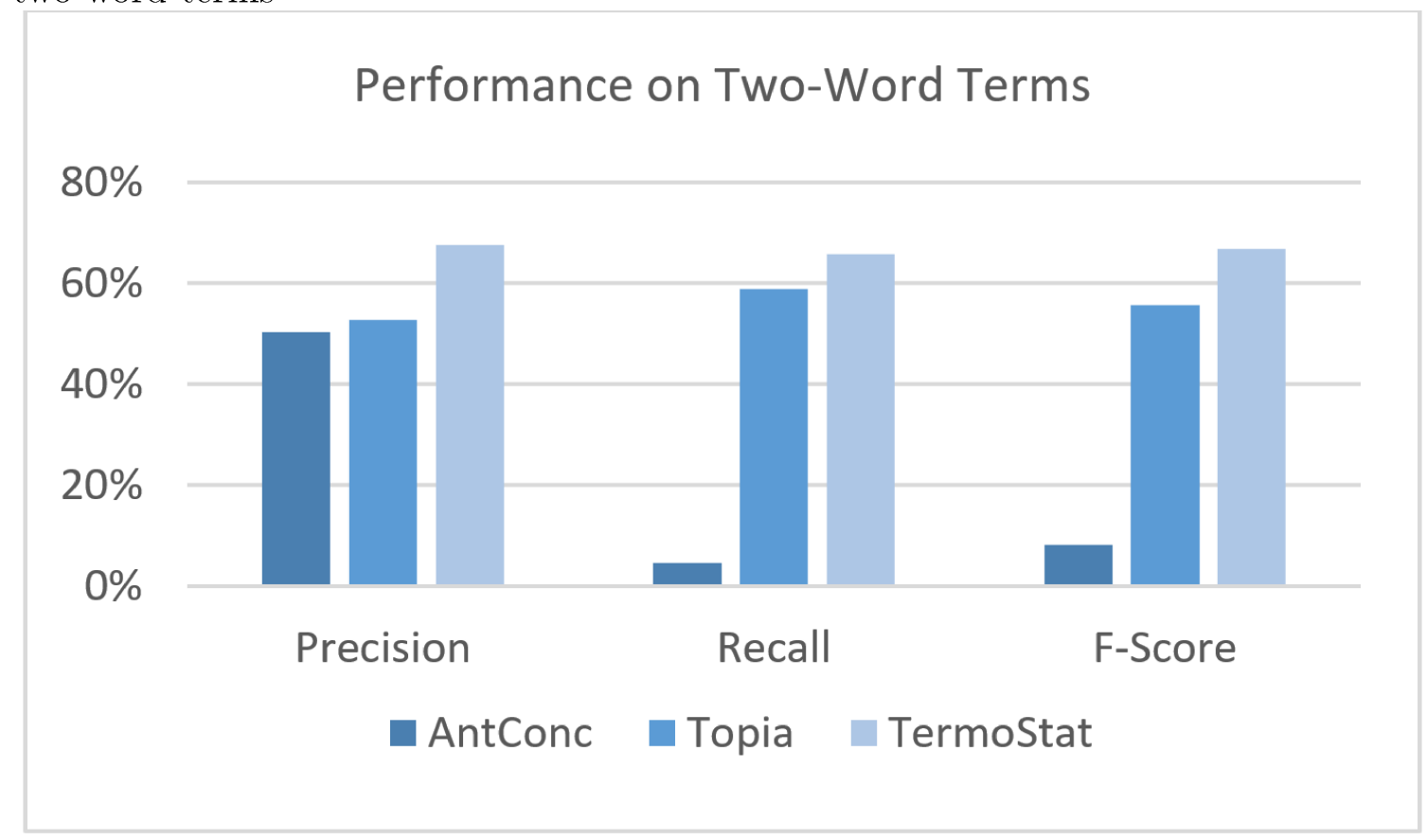

the highest scores due to its solid statistical measure, good performing POS tagger, and its extraction patterns. Topia achieved higher than AntConc n-grams for oneand two-word categories. Nevertheless, Topia's low performance in extracting 3-word and 4-word terms coupled with a somewhat constant precision of AntConc n-grams over the 4 categories, gave AntConc the second place in overall performance. Topia had a better overall recall score than AntConc, but a worse precision.

\begin{tabular}{|c|c|c|c|}
\hline Overall Performance & Precision & Recall & F-Score \\
\hline AntConc & $47 \%$ & $45 \%$ & $46 \%$ \\
Topia & $32 \%$ & $55 \%$ & $41 \%$ \\
TermoStat & $\mathbf{5 5 \%}$ & $\mathbf{6 4 \%}$ & $\mathbf{5 9 \%}$ \\
\hline
\end{tabular}

Table 3.1: Overall performance of the term extraction tools in our corpus 
Figure 3.6: Comparison of the performance of the term extraction tools in extracting three-word terms.

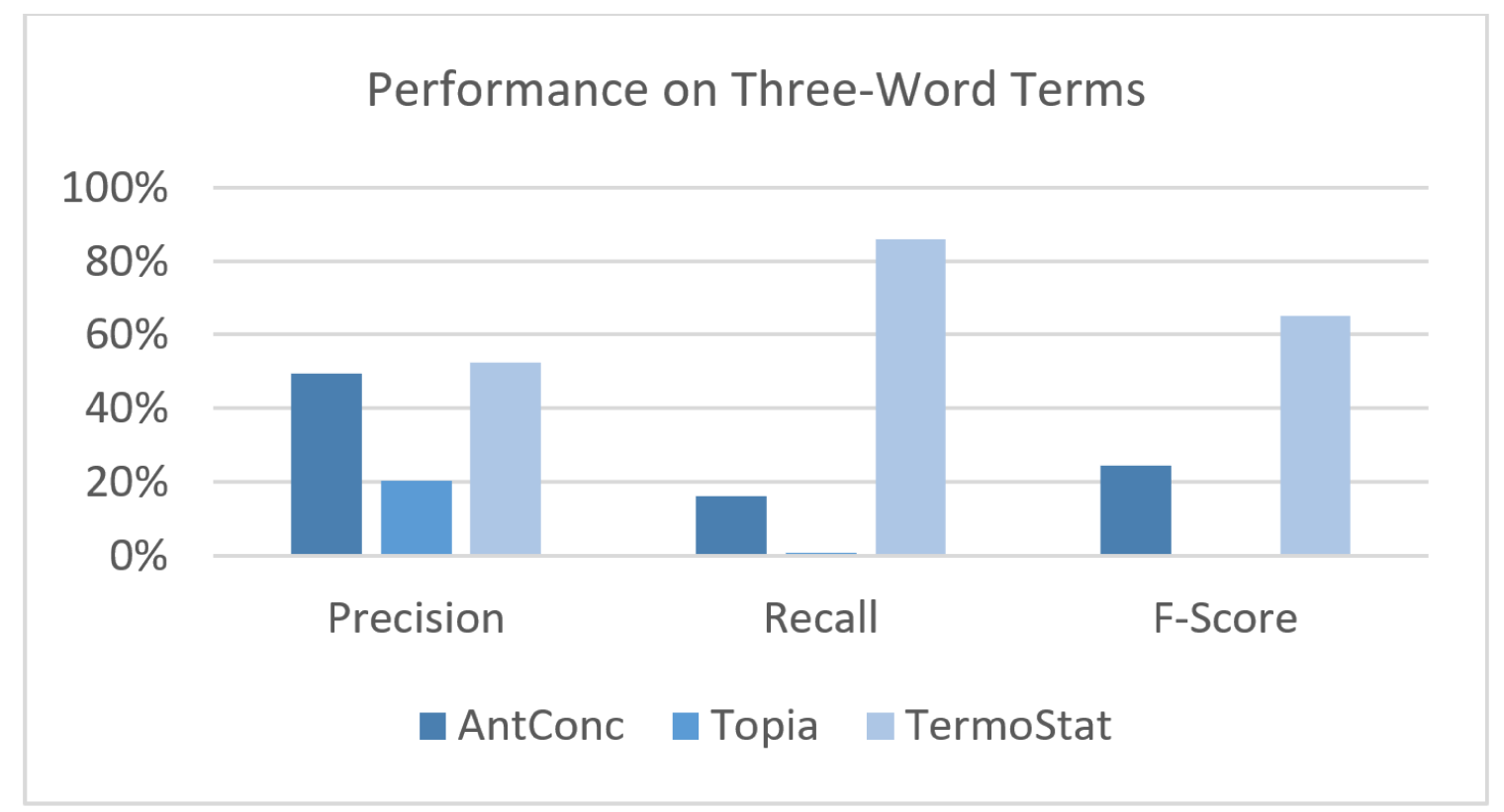

Figure 3.7: Comparison of the performance of the term extraction tools in extracting four-word terms.

\section{Performance on Four-Word Terms}

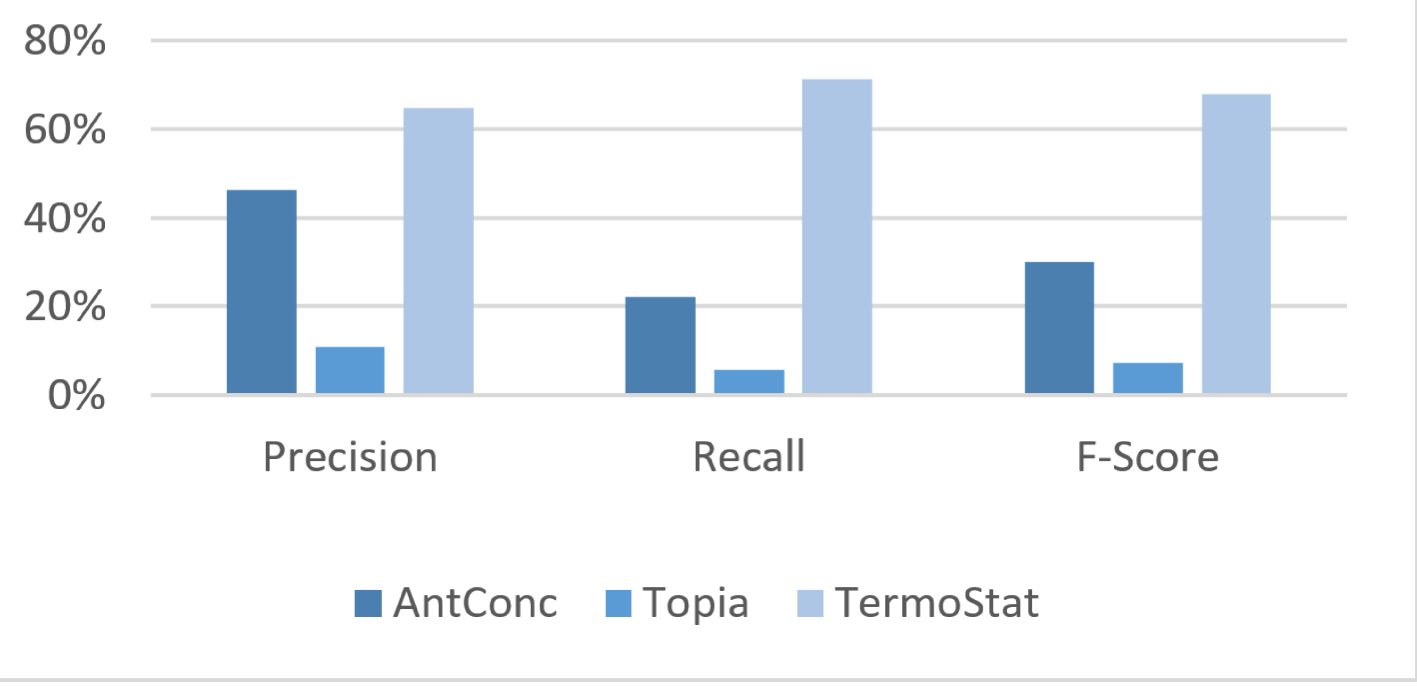


Table 3.2 presents the agreement ${ }^{18}$ scores in percentage between the annotators as provided by the Term Evaluator. The bottom row shows the overall agreement for each tool across all categories. Annotators agreed on AntConc results the most, followed by Topia, and TermoStat. We consider our data non-sequential and have computed Cohen's kappa statistics for inter-annotator agreements (Carrillo et al., 2014).

\begin{tabular}{|c|c|c|c|}
\hline Inter-annotator agreement & AntConc & Topia & TermoStat \\
\hline 1-word & $92 \%$ & $85 \%$ & $78 \%$ \\
2-word & $93 \%$ & $83 \%$ & $73 \%$ \\
3-word & $87 \%$ & $84 \%$ & $66 \%$ \\
4-word & $73 \%$ & $81 \%$ & $68 \%$ \\
Overall & $86 \%$ & $84 \%$ & $72 \%$ \\
\hline
\end{tabular}

Table 3.2: Agreement scores between annotators for each word category and each tool, and overall agreement

Table 3.3 shows the kappa statistics for agreement scores for each word category and each tool. The kappa values are consistent with Term Evaluator's agreement scores in that the highest overall agreement belongs to AntConc, followed by Topia, and TermoStat comes last (see table 3.3). It is noteworthy that the kappa value for Topia for the 4-word category is very low, which coincides with the lowest performance in table 3.1. Low agreements may often occur in term extraction (Vivaldi and Rodríguez, 2007; Loginova et al., 2012), but this specific case is due to the very high $\mathrm{P}(\mathrm{e})$ value for Topia in the 4 -word category, which is equal to 0.81 . This partially originates from the tool's noisy output for this word category which resulted in a very low correctness score $(7 \%)$ for one of the annotators.

\footnotetext{
${ }^{18}$ Since non-expert judges were used in this study, when computing agreement scores we collapsed the categories Non-Technical, Wrong Term (i.e. non-terms/No), and Not Sure (see section 3.6 figures 3.1 and 3.2) into one, called incorrect.
} 


\begin{tabular}{|c|c|c|c|}
\hline Kappa Statistics & AntConc & Topia & TermoStat \\
\hline 1-word & 0.84 & 0.70 & 0.53 \\
2-word & 0.86 & 0.67 & 0.40 \\
3-word & 0.73 & 0.54 & 0.48 \\
4-word & 0.47 & 0.05 & 0.36 \\
Overall & 0.71 & 0.62 & 0.49 \\
\hline
\end{tabular}

Table 3.3: Cohen's kappa statistics for annotators per word category and per tool, and overall

We investigated the cause of disagreements between the two annotators, by asking them to discuss the cases of disagreement. Annotator 1 evaluated fewer terms as "Not Sure", while Annotator 2 was more uncertain about whether the corresponding terms pertained to mathematics. Annotator 2 had an issue with 2-word terms in which one word was a mathematics word and the other was not.

Examples include the candidate terms "combined function" and "resultant velocity". Another source of confusion was the signs and symbols that cannot be considered words. One annotator marked many of them as "Non-Term", and the other as "Not Sure". Numerals, such as "ii" also caused problems: Annotator 1 marked them as "Not Sure", while Annotator 2 considered them mathematics terms.

In a follow up experiment, we asked our human judges to annotate all the 4011 terms extracted by TermoStat, in order to measure how much time they save by using our Term Evaluator tool. From these, 475 terms had already been filtered out by our tool because they started or ended in stop words, 501 had automatically been marked as good terms because they were found in the domain dictionaries included in the tool, and 368 had also automatically been marked because they were in the secondary list of terms already evaluated as correct terms in the previous experiments. This left 2667 terms to be annotated, which represents a saving of $33 \%$. 


\subsection{Summary}

This chapter offered a gold standard for the automatic terminology extraction task in the mathematics education domain. We constructed a mathematics education corpus and built a dataset by a post-hoc annotation task. We presented an agreement analysis between our annotators and evaluated the performance of the tested tools on this dataset for different categories of n-gram terms. This work substantially facilitates future studies in the above-mentioned domain, as well as the creation and evaluation of the future methods, including the method proposed in the next chapter. 


\section{Chapter 4}

\section{Distributed Specificity}

The present chapter proposes two flavors of a novel method that integrates distributed representation with terminology extraction. Both both versions of the method assess the specificity of a word (unigram) to the target corpus by leveraging its distributed representation in the target domain, as well as in the general domain. The first adopts this distributed specificity as a filter, and the second directly applies it to the corpus. The filter can be mounted on any other ATE method, allows merging any number of other ATE methods, and achieves remarkable results with minimal training. The direct approach does not perform on par with the filtering approach, and shows more thirst towards data. However, it emphasizes that using distributed specificity as word representation, little data is required to train an ATE classifier. This encourages more minimally-supervised ATE algorithms in the future. 


\subsection{Introduction}

The terminology of a domain encodes the existing knowledge in that domain. Hence understanding and interpreting a message belonging to a domain cannot be fully achieved without knowing its terminology. This makes Automatic Terminology Extraction an important task in Natural Language Processing. Furthermore, as a significant component of any academic subject is its terminology, knowledge of the terminology of a field enables students to engage with their discipline more effectively by enhancing their ability to understand the related academic texts and lectures, and allowing them to use the subject-specific terminology in their discussions, presentations, and assignments. Therefore, generating lists of terms specific to various fields of study is a significant endeavor. However, these lists have often been generated manually or through corpus-based studies, which are time consuming, labor-intensive, and prone to human error. This can be facilitated by a great extent with high-performance automatic term extraction.

ATE methods have been conventionally classified as linguistic, statistical, and hybrid (Cabre-Castellvi et al., 2001; Chung, 2003). Linguistic methods implement formal rules to detect terms; statistical methods exploit some measures based on relative frequency of terms in general and target corpora by means of which they can tell apart a term from a word in its generic sense; and, the hybrid methods combine the advantages of both of these techniques (Frantzi et al., 1998; Park et al., 2002; Drouin, 2003; Chung and Nation, 2004; Yoshida and Nakagawa, 2005; Vu et al., 2008; VRL, 2009; Yang et al., 2010; Zervanou, 2010; Broß and Ehrig, 2013; Conrado et al., 2013). These methods often regard words in a document as atomic elements; that is, they are manifested as their symbolic alphabetical form in the algorithm and/or as some 
measure of their relative frequency. But, in a distributed approach each word has $n$ real-valued components, as opposed to a single linguistic form or a termhood score ${ }^{1}$. The idea is that such finer-granularity may grant more access to the information that a word contains, potentially resulting in a better detection of terms in a document.

Here $n$ is often between 50 and 1000 for different types of word embeddings. Other vector space models such as Latent Semantic Analysis (LSA) and Latent Dirichlet Allocation (LDA) have the same requirement of choosing the dimensionality of the reduced vector space and the number of topics, respectively. Refer to section 2.4.3 for further details regarding the LSA and LDA methods.

In addition to the richer representation, the rise of distributed methods in NLP, especially the recent word embeddings surge, makes it relevant to explore the ways current model architectures may fit into the automatic terminology extraction picture. However, what makes them particularly appealing is their computational efficiency and scalability as compared to the available alternatives, including LSA and LDA (Mikolov et al., 2013b).

We present a simple method that harnesses the rich distributed representation acquired by a log-bilinear regression model GloVe (Pennington et al., 2014), as well as the efficiency of log-linear models with $\mathrm{CBOW}^{2}$ and skip-gram architectures ${ }^{3}$ (Mikolov et al., 2013b), which is a step forward towards language-independent ${ }^{4}$ ATE as it requires no language specific annotation (e.g., POS tags, parse trees, or language

\footnotetext{
${ }^{1}$ Termhood is the degree of a linguistic unit being related to a domain-specific concept (Kageura and Umino, 1996)

${ }^{2}$ Continuous Bag Of Words

${ }^{3}$ See section 2.4.4 for details on these architectures.

${ }^{4}$ It is noteworthy that our filtering method (see section 4.4.2) does still utilize language-specific information provided by the previous methods.
} 
specific patterns). In our method, the GloVe model is used to preserve the global ${ }^{5}$ scope of a word in a general corpus (i.e., its general sense(s)) and the CBOW or the skip-gram model is used to capture the local scope for a word in a technical corpus (i.e., its technical usage).

Our target corpus belongs to high school mathematics domain. Unigram terms were particularly challenging during annotation, partially due to the notable presence of unigram terms that sat on the boundary of termhood. These unigrams were particularly difficult as they did belong to the mathematical domain but not necessarily to the high school sub-domain (v.i. section 4.5) A significant portion of technical terms is composed of multi-word expressions. Although association measures such as local mutual information can be used along with embeddings to address phraseness (Wang et al., 2015), these measures enforce a heterogeneous secondary layer of computation. We, in contrast, prefer to explore the path to a unified distributed approach that represents unigram ${ }^{6}$ terms and multi-word expressions alike. This demands a separate study devoted to the suitability of deeper networks to include representation and classification of multi-word terms. Hence, our methods in this chapter focus on the unigram term extraction task.

Our first approach is a filter mounted on top of a previously-developed hybrid term extraction algorithm, namely, TermoStat (Drouin, 2003; Le Serrec et al., 2010) along with two simpler methods (refer to section 4.4 for further details). TermoStat has been tested in the previous chapter on the mathematics domain where it performed

\footnotetext{
${ }^{5}$ We use the terms "global" and "local" with a different sense from Pennington et al. (2014). They used "global" to denote a model that captures a wider set of co-occurrence statistics being computed globally (e.g., document-wide) such as in LSA, as compared to the "local" methods that use a relatively small context window for co-occurrence computation such as $\mathrm{CBOW}$ and skip-gram (Mikolov et al., 2013b) or similarly vLBL and ivLBL (Mnih and Kavukcuoglu, 2013).

${ }^{6}$ Single words
} 
well on the extraction of multi-word expressions, but with lower precision on unigram terms (Inkpen et al., 2016). Our second approach is a direct application of our method to the text of the corpus. The present work is an attempt to improve unigram term extraction for the mathematics domain, specifically mathematics textbooks.

Our method successfully applies neural network word embeddings to the terminology extraction task. Our filter can be combined with any term extraction algorithm(s) for any non-polysynthetic ${ }^{7}$ language and any domain.

\subsection{Related Work}

As described in section 4.1, ATE approaches traditionally fall into three categories, namely, linguistic, (unsupervised) statistical ${ }^{8}$, and hybrid methods (Cabre-Castellvi et al., 2001; Chung, 2003). These ATE methods have been applied to both monolingual and multilingual corpora (Ljubešić et al., 2012). Linguistic methods apply hand-coded rules to the target corpus to extract technical terms. Statistical methods are often unsupervised and apply some measure of relative frequency to a technical target corpus, a reference (general) corpus and sometimes a (contrastive) corpus from another domain, to identify the existing terms in the technical corpus (Frantzi et al., 1998; Chung, 2003; Vu et al., 2008; Conrado et al., 2013). Hybrid methods combine statistical and linguistic methods to extract terminology from a target corpus and often perform very well (Drouin, 2003; Le Serrec et al., 2010; Ismail and Manandhar, 2010; Vintar, 2010). The above-mentioned approaches, in contrast to the methods put forth in this chapter, regard words as atomic units represented by their linguistic

\footnotetext{
${ }^{7}$ A polysynthetic language has a richer morphology than syntax, where the words are much longer and can convey full sentence-like messages

${ }^{8}$ These statistical methods are distinct from statistical learning approaches.
} 
forms and their statistical scores that indicate the likelihood that they are terms. They may, however, implement rules associated with some linguistic features (e.g., their POS tags, their position in the POS sequence, their position in the parse tree, phrase, and/or in the sentence). These linguistic rules make an algorithm languagedependent and even sometimes to some degree domain-dependent. On the contrary, our method, if used independently, can be used for any non-polysynthetic language and for any domain as long as a suitably-large domain-specific and general corpora are available. In this study, we apply our method in combination with other methods as well as independently. It can still be regarded as a step towards a language independent ATE algorithm that benefits from latent linguistic information encoded in the vectors used (see section 4.4 for further details on the method) in comparison to purely statistical methods that do not capture such information.

Supervised methods have been recently ${ }^{9}$ designed for terminology extraction. Nazar and Cabré (2012) used examples of terms from the domain of interest and a reference corpus of general language, which represent positive and negative examples of terms, and a three-level (i.e., syntactic, lexical, and morphological) learning algorithm to detect the terms. They used the frequency distribution for POS tag sequences at the syntactic level. At the lexical level, they accounted for the frequency of the lexical units within the terms (word forms, as well as lemmas). Finally, at their morphological level, from each word type they extracted initial and final character $n$-grams where: $1 \leq n \leq 5$ (Nazar and Cabré, 2012). Their term extractor is an online web-based system that is constantly being updated when used by terminolo-

\footnotetext{
${ }^{9}$ There has been earlier supervised work in keyword/keyphrase extraction such as Turney (2000), as opposed to terminology extraction which is the topic of this chapter. While keyword extraction is the task of extracting only a few keywords in a text, terminology extraction aims to detect the terms, usually from a large domain corpus.
} 
gists. More recently, Conrado et al. Conrado et al. (2013) achieved state-of-the-art performance for unigram term extraction in Brazilian Portuguese using supervised learning algorithms and a rich feature set. They used eight linguistic features, seven statistical features, and four hybrid features in their method. The present work would be the next phase for these supervised methods, since we move closer to a rich, language-independent, resource-independent, and fully data-driven representation. It is worth noting that modern word embeddings have been successfully employed in many tasks, including the related areas of keyphrase extraction (Wang et al., 2015) and aspect term extraction ${ }^{10}$ (Yin et al., 2016); nevertheless, in the present study they are leveraged for the general terminology extraction task.

\subsection{Corpus}

As mentioned in the previous chapter, the domain corpus that we used for the purpose of this study is comprised of 5 English high school mathematics textbooks used in Ontario, Canada (Small et al., 2005, 2007a,b; Kirkpatrick et al., 2007; Crippin et al.). This domain was chosen as an effort to facilitate mathematics education for high school students as the knowledge of terminology plays a pivotal role in learning the mathematical concepts. In addition the content of the books are often updated and there is a need for accurate and automatic extraction of terms as manual extraction of terms is labor-intensive and prone to human error and inconsistencies. These textbooks cover grades 9 to 12 , and include topics on functions, calculus, and vectors. They were originally in PDF format from which the raw texts were extracted. They

\footnotetext{
${ }^{10}$ Aspect term extraction is the task to identify the aspect expressions which refer to a product's or service's properties or attributes, from customer reviews (Pontiki et al., 2014, 2015; Yin et al., 2016).
} 
then were concatenated into a corpus consisting of $1,127,987$ tokens. The corpus has been sanitized by removing the bibliography.

\subsection{Methodology}

We introduce a novel ATE method called distributed specificity. Distributed specificity employs a specificity vector to determine if a given word is a term in the corpus. This method can be thought of as an extension to the specificity approach that adopts distributed representation as opposed to mere relative frequencies. We investigate two different approaches of distributed specificity for ATE. Our first approach mounts as a filter on top of other ATE algorithms. Our second approach applies distributed specificity directly to the corpus as an independent ATE algorithm.

\subsubsection{Specificity Vectors}

Both of the implemented approaches exploit specificity vectors in their algorithms. The basic idea is: if a candidate term in a technical corpus is being used in a distinctly different manner and context than in a general corpus, then it is likely to be a term. This is similar to the premise of traditional statistical ATE methods with specificity/termhood scores, except that those models carry less local information such as syntactic behavior.

For each Candidate Term (CT), two separate embedding vectors are constructed and then concatenated into a specificity vector. One is a global vector pre-trained on general corpora, and the other is a local vector trained on the target corpus from which the terms are to be extracted. Each of these two vectors portrays distinct 
regularities about the $\mathrm{CT}$ at hand, as discussed below.

The idea behind using a general global vector is to encapsulate the behavior of the $\mathrm{CT}$ in its generic sense(s), the intuition being that the generic sense(s) have a predominant presence in general corpora and will, therefore, dominate the vector. We use as our global vectors the pre-built GloVe vectors, created by Pennington et al. $(2014)^{11}$. These global vectors are of 50 dimensions and were built on Wikipedia $2014+$ the Gigaword 5 corpus; that is, approximately 6 billion tokens. GloVe is a log-bilinear regression model, which views the modeling task as a least squares problem to minimizes $J$. Pure co-occurrence, however, does not properly account for co-occurrences that take place rarely or do not occur at all (i.e., zero counts in the matrix) which is a great source of noise in the data. This is accommodated by a weighting function in the model. More formally the model is:

$$
J=\sum_{i, j=1}^{V} f\left(X_{i j}\right)\left(\mathrm{w}_{i}^{T} \tilde{\mathrm{w}}_{j}+b_{i}+\tilde{b}_{j}-\log X_{i j}\right)^{2}
$$

where $V$ is the size of the vocabulary, $X$ is the word-word co-occurrence counts matrix, $X_{i j}$ is the number of times word $j$ occurs in the context of word $i, f\left(X_{i j}\right)$ is the weighting function, $\mathrm{w}_{i}$ and $\tilde{\mathrm{w}}_{j}$ are two separate context word vectors and their sum constructs the final GloVe vector, and finally $b_{i}$ and $b_{j}$ are biases for their corresponding word vectors. GloVe has been shown to adequately reflect both semantic and syntactic regularities in the data Pennington et al. (2014); we require both for our global embeddings.

In contrast to global embeddings, technical local embeddings are built on the domain corpus. These vectors are valuable since they capture the behavior of the

\footnotetext{
${ }^{11}$ Available at: http://nlp.stanford.edu/projects/glove/
} 
candidate terms in the technical domain. To construct the local embeddings, we use two neural network architectures introduced by Mikolov et al. (2013b) on our corpus (discussed in section 4.3 above), namely, the CBOW and the skip-gram architectures. CBOW and skip-gram are efficient algorithms trained by stochastic gradient descent and backpropagation (see section 2.4.4 for further details on these architectures). Below are their computational complexities, respectively:

$$
\begin{gathered}
Q_{C B O W}=N \times D+D \times \log _{2}(V) \\
Q_{\text {skipgram }}=C \times\left(D+D \times \log _{2}(V)\right)
\end{gathered}
$$

where $N$ is the number of context words, $D$ the vector dimensionality, $V$ the vocabulary size, and $C$ is roughly the maximum distance for the context from the target word. $\mathrm{CBOW}$ is trained to predict a target word based on its surrounding words, and the skip-gram model is trained to predict the surrounding words given a single word. The CBOW architecture tends to have better performance in discovering syntactic regularities as compared to semantic regularities, whereas the skip-gram architecture tends to have a higher performance in finding semantic regularities rather than syntactic ones (Mitkov et al., 2012; Pennington et al., 2014). Because we are dealing with unigram terms and not multi-word terms at this stage, we expect a skip-gram filter to outperform a CBOW filter. We used the gensim ${ }^{12}$ implementation Řehưřek and Sojka (2010) of word $2 \mathrm{vec}^{13}$ to build vectors of 100 dimensions with context window of size 5 and minimum frequency of 5 . The rest of the parameters were left at their default values.

\footnotetext{
${ }^{12}$ Available at: https://radimrehurek.com/gensim/models/word2vec.html

${ }^{13}$ Available at: https://code.google.com/archive/p/word2vec/
} 
We did not perform hyper-parameter optimization for our architecture. The choice of 50d for GloVe was due to computational feasibility and memory efficiency. The hyper-parameters for the local embeddings were chosen intuitively to capture a reasonable context by the context window size and reduce the vocabulary size to be more manageable and less computationally expensive.

\subsubsection{Filtering Approach}

Our filter merges the results of three tools that we used for terminology extraction. We improved the performance of these tools by adding specificity vectors representing words, coupled with a classifier. For each CT extracted by TermoStat, a specificity vector is constructed.

The specificity vector is then fed to the classifier to make the final decision. The components of the specificity vector are treated as the input feature vector for the classifier. We experimented with several classification algorithms. Following Conrado et al. (2013) (see section 4.2 for more details), we used JRip ${ }^{14}$, Naïve Bayes, J48 ${ }^{15}$, and $\mathrm{SMO}^{16}$ (Platt, 1998). We also tested a few other classifiers of our choice to find the most suitable ones for the task, including logistic regression, Multi-Layer Perceptron (MLP) and PART ${ }^{17}$. Default parameters were used for these classifiers. We employed the Weka implementation of all the above-mentioned classifiers. We tested all the classifiers for both the CBOW and the skip-gram architecture.

As mentioned above, our approach operates on the results of three other term ex-

\footnotetext{
${ }^{14} \mathrm{~A}$ rule induction classifier

${ }^{15} \mathrm{~A}$ decision tree algorithm. We used it with confidence factor of 0.25 .

${ }^{16}$ A Support Vector Machine classifier from Weka

${ }^{17}$ Another rule induction algorithm
} 
traction tools. The first is a full-fledged hybrid ATE tool called TermoStat ${ }^{18}$ (Drouin, 2003). It computes the specificity of a term in a corpus with reference to a general corpus by means of a statistical test developed to target highly specific technical terms (see Drouin (2003) for more details on the statistical test). There are three outcomes: $\mathrm{SP} 0, \mathrm{SP}+$, and $\mathrm{SP}-$, meaning the observed frequency in the corpus is consistent, significantly higher, or significantly lower, respectively, with regard to the reference corpus. SP+ constructs a corpus-specific vocabulary which are called Specialized Lexical Pivots or SLPs for short. TermoStat uses POS-tag patterns to detect head nouns and term phrases. TermoStat extracted 903 terms and the top 500 were picked for the experiments. The second term extraction tool is Topia ${ }^{19}$. We augmented it by a filter that removed all the candidate terms that had fewer than 3 letters and took out numbers or special characters from candidate terms. Topia uses the majority POS tag for each word, and applies only a frequency threshold to extract terms. It extracted 2,972 terms but the top 500 were selected for the experiments. Third, we extracted most frequent unigrams using AntConc ${ }^{2021}$ Anthony (2012), but only the first 500 candidates were selected and we filtered out all the stop-words.

Figure 4.1 illustrates our overall filtering system. First, the term extraction tools operate on the target corpus. Then, the resultant CTs from all of them are pooled together (with no repetition) and are fed to the filter. The filter uses the local vectors trained on the technical corpus as well as the global vectors trained on the general corpus to represent the received CTs in 150 dimensional vectors. These vectors are then forwarded to the classifier to tell terms from non-terms. The highest-performing

\footnotetext{
${ }^{18}$ Available at: http://termostat.ling.umontreal.ca/

${ }^{19}$ topia.termextract 1.1.0 library available at: https://pypi.python.org/pypi/topia.termextract/

${ }^{20}$ Available at: http://www.laurenceanthony.net/software/antconc/

${ }^{21}$ AntConc has a keyword extraction module but no term extraction module.
} 
classifier is then found and used to initialize the system. We compare the results of our filtering approach with the results received from each of the term extraction tools used in isolation.

Figure 4.1: The figure depicts the overall system architecture of our approach

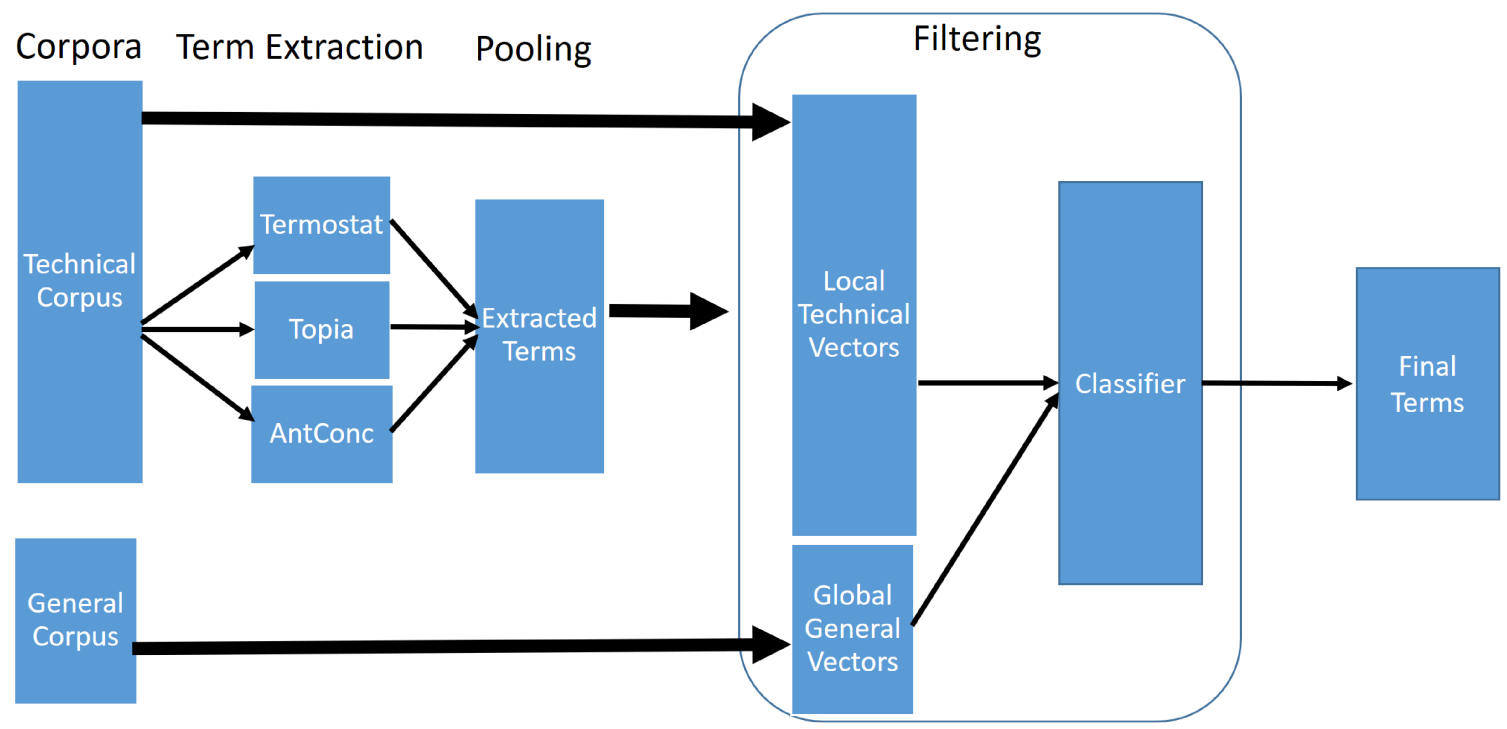

\subsubsection{Direct Approach}

Our direct approach uses distributed specificity alone to determine the termhood of a word in the corpus. Similar to the filtering approach, a specificity vector comprises a local vector built by either of the CBOW and skip-gram architectures, and a global GloVe vector. Specificity vectors are constructed for every word in the target corpus with frequency $f \geq 5$. The best performing classifiers from the filtering approach are then trained on these specificity vectors to tell terms and non-terms apart. A marked characteristic of this approach as compared to the filtering approach is that the data will be highly imbalanced; that is, the number of non-terms in the corpus will be far 
greater than the number of terms.

\subsection{Annotation}

To evaluate the performance of our method, two human annotators judged the terms extracted by the term extraction tools. The annotators used Term Evaluator ${ }^{22}$ Inkpen et al. (2016), a software program for annotating and evaluating terminology extraction, to judge the results (v.i. section 3.6). The annotators were asked to use their background knowledge of mathematics as the primary source of their judgment. In case of confusion, they could consult a mathematics dictionary of their choice (see section 3.7 for the details of the annotation process. The annotations had kappa agreement scores of $\mathrm{k}=0.70$ for Topia, $\mathrm{k}=0.84$ for AntConc and, $\mathrm{k}=0.53$ for TermoStat. AntConc has the highest agreement among the ATE tools. This is due to the frequency effect, that is, the high frequency words in the corpus were deemed most familiar by the annotators, hence, their termhood was easier to approve or reject by them. The annotation resulted in a dataset consisting of 954 instances with 325 positive and 629 negative cases, by which we assess the performance of the systems used in this study. Only the terms that the annotators agreed upon their labels found their way into the dataset. The most frequent term in the dataset is function with 9,505 occurrences in the corpus, and the least frequent term is miligram with only 3 occurrences. The mean occurrence of the terms in the positive set is 504.01 and the mean occurrence for the negative instances is 82.39. In this study, we compute relative recall as the dataset does not include any annotation from our direct approach algorithm. The reason for resorting to relative recall is that having annotators go

\footnotetext{
${ }^{22}$ Available at: https://sourceforge.net/projects/termevaluator/
} 
through the entire corpus to compute recall is time-consuming and labor-intensive at this phase of the project. A source of challenge to the annotation task was the fact that it is not always straightforward to determine if a mathematics term should be considered a term in a high school mathematics textbook. For instance, the word division does have a technical mathematical sense, which makes it a term, however, it may not automatically be considered a term in the high school mathematics educational context. Another source of challenge was polysemy, for instance, words such as cross, dot, tale, arch, and right were not considered to be terms by the annotators but words such as point, solution, expression, height, interval, and term were marked as correct terms. These issues have had a major presence in the unigram terminology annotation as compared to the multi-word expressions in our corpus and are a contributing factor to making this category challenging for the ATE algorithms used.

\subsection{Experiments and Results}

First, we aim to find the best-performing classifier(s), out of the ones tested, to be used in our system for each of the two architectures (CBOW and skip grams). We noticed that three classifiers, namely, SMO, logistic regression, and the MLP consistently outperformed the rest of the classifiers we examined (the full list is provided in section 4.4). JRip performed well, but its performance was consistently lower than the abovementioned three classifiers. It should be noted, however, that we also have a greater dimension size for our vectors than Conrado et al. (2013), that is, 150 versus 19. Also the nature of the vectors is different in that they used feature vectors but we used embeddings. Nevertheless, we only show the results for these three classifiers. Figure 4.2 depicts the classifiers' performance with specificity vectors where the local 
vectors are trained with the CBOW architecture, and Figure 4.3 depicts the classifiers' performance with specificity vectors where the local vectors are trained with the skip-gram architecture. The classifiers' performance is presented as a function of the number of observed instances ${ }^{23}$ (the amount of training data used), and the classifiers are tested on the rest of the instances (954 minus the number of observed instances). Instances are chosen randomly for training with a positive/negative ratio proportional to the dataset (i.e., $1 / 2$ respectively). All of the instances in the entire dataset are unique candidate terms. The performance is measured by F-measure (F1-score) in the figures. We compute only relative recall throughout the experiments at this stage.

Figure 4.2: Performance (F-measure) of the classifiers on local-global vectors with CBOW local vectors as a function of number of observed instances

CLASSIFIERS' PERFORMANCE ON SPECIFICITY WITH LOCAL CBOW

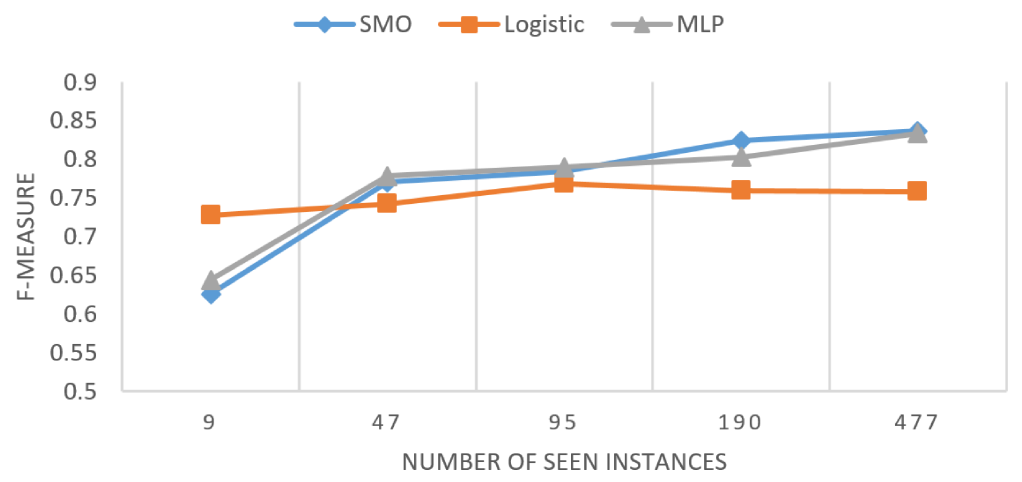

As shown in Figures 4.2 and 4.3, the CBOW architecture with the logistic regression classifier generalizes quite well with as little data as only 9 instances. However,

\footnotetext{
${ }^{23}$ The numbers shown on the $X$ axes of Figures 3 and 4 (i.e., 9, 47, 95, 190, and 477) are the results of splitting training and test data such that the training data is approximately $1 \%, 5 \%$, $10 \%, 20 \%$, and $50 \%$ of the entire dataset respectively. The most notable improvement is when we increase the training set from 9 to 47 and that is only $4 \%$ variation in the size of the test set but $10 \%$ improvement of performance on average for specificity vectors with local CBOW (Figure 4.2) and an average of $13 \%$ improvement of performance for specificity vectors with local skip-gram (Figure $4.3)$.
} 
Figure 4.3: Performance (F-measure) of the classifiers on local-global vectors with skip-gram local vectors as a function of number of observed instances

CLASSIFIERS' PERFORMANCE ON SPECIFICITY VECTORS WITH LOCAL SKIP-GRAM

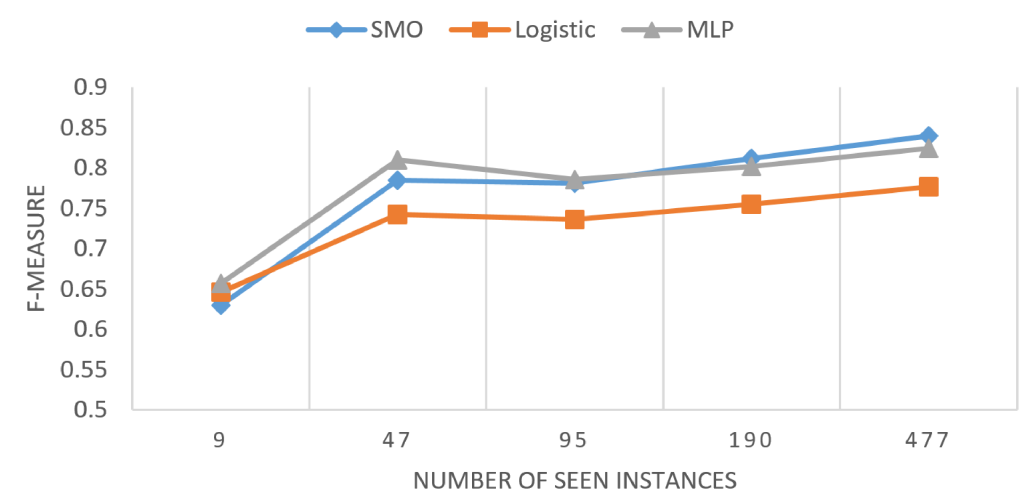

as soon as we add more instances, the MLP and SMO take the lead. However, the logistic regression classifier shows less improvement when subjected to more training data. Overall, we did not notice any considerable difference between the skip-gram and the CBOW architectures across the classifiers used for the purpose of unigram term extraction.

In practice, we prefer to show the system as little data as possible since extracting a few high-precision terms is relatively easy in real-world ATE; hence, we choose the local CBOW architecture with logistic regression classifier (trained on only 9 instances) as one configuration that was our fastest learner. In addition, we choose the local skip-gram architecture coupled with MLP as another configuration for the next experiment, because it performed best among those trained on up to 47 instances. We compare these two system configurations with a baseline and the initial term extraction tools, all tested on 907 (i.e., 954 - 47) remaining instances that are unseen to all of the systems under experiment. Table 4.1 compares the results of our system 
in unigram term extraction with individual term extraction tools and a frequency baseline that uses a stop-word filter (refer to section 4.4 for further details on the tools and the baseline). The results show that both of our system configurations achieve a substantial improvement over the other tools.

Table 4.1: Results of two configurations of our system, Filtering9 (using CBOW local vectors and the logistic classifier) and Filtering47 (using skip-gram local vectors and MLP), with the term extraction tools used in isolation and with a frequency baseline

\begin{tabular}{|c|c|c|c|}
\hline Method & Precision & Recall & F-measure \\
\hline TermoStat & 0.371 & 0.528 & 0.436 \\
Improved Topia & 0.426 & 0.702 & 0.552 \\
Frequency + Stopword Filter & 0.407 & 0.514 & 0.454 \\
Filtering9 (CBOW) + Logistic & 0.728 & 0.734 & 0.728 \\
Filtering47 (skip-gram) + MLP & $\mathbf{0 . 8 0 9}$ & $\mathbf{0 . 8 1 1}$ & $\mathbf{0 . 8 1 0}$ \\
\hline
\end{tabular}

We use the highest performing classifiers from our filtering approach for our direct approach, namely, SMO, logistic, and MLP. The direct approach scans and tokenizes the corpus, and then creates a vocabulary. Afterwards, for each vocabulary item, the distributed specificity measure is applied. This results in an imbalanced dataset. We did not include items with frequency $f<5$ in the vocabulary. The resultant size of the vocabulary is 9,916 entries, only 325 of which are terms according to our annotation. It is worth noting that our direct approach may be at a disadvantage because the annotation was conducted on the outcome of the other tools, whereas our direct approach may extract terms that were not recognized by the other methods and therefore unjustly penalized as false positives, since we treat every vocabulary item that is not in our list of positive terms as non-terms. That makes most of the vocabulary negative instances. Nevertheless, we present the results of the direct approach in table 4.2 below. The classifiers presented in the table have been trained 
on only 6 positive and 18 negative instances. These instances have been randomly sampled 10 times. The results are averaged over all the training samples for each classifier. Increasing the number of negative and positive instances did not improve the performance. With the selected number of training instances, specificity vectors with CBOW local vectors consistently resulted in a higher F-measure. The direct approach came close to TermoStat which is a robust hybrid method, but could not outperform the earlier methods and falls much behind our filtering approach. Regardless, the results of the direct approach signify the fact that, given distributed specificity representations, an ATE classifier can be trained with very little training data. This opens the door for more supervised ATE algorithms to appear. This observation is consistent with the results of our filtering approach.

Table 4.2: Results of 3 configurations of our direct approach. Three classifiers were trained on 6 positive and 18 negative training instances

\begin{tabular}{|c|c|c|c|}
\hline Method & Precision & Recall & F-measure \\
\hline MLP (CBOW) & 0.278 & 0.182 & 0.220 \\
SMO (CBOW) & $\mathbf{0 . 3 6 0}$ & 0.418 & $\mathbf{0 . 3 8 6}$ \\
Logistic (CBOW) & 0.302 & $\mathbf{0 . 5 1 8}$ & 0.382 \\
\hline
\end{tabular}

In table 4.2, MLP shows the lowest precision and recall due to the small number of training examples. This affects MLP more than the two other classifiers, since it is a simple deep neural network, and these networks often work well when trained on substantially greater number of instances. 


\subsection{Summary}

This chapter presented a novel algorithm based on distributed representation, that substantially improves on the efficacy of automatic terminology extraction algorithms. It can also be used to combine multiple ATE methods. We showed that this novel method, although supervised in nature, can be minimally trained to achieve its high performance. Such quality is essential for ease of application to other domains. We also showed the potential of applying distributed specificity directly to text. 


\section{Chapter 5}

\section{Cross-Domain Distributed}

\section{Automatic Terminology Extraction}

In chapter 4 , we introduced novel algorithms that in addition to exploiting the information in frequency variances between general and technical corpora, took into account the syntagmatic and paradigmatic variances between the two, resulting in a representation that is syntactically and semantically more informative across domains. We also witnessed that with minimal supervision provided by a few seen instances (training examples), high-performing classifiers could be successfully trained to outperform previous methods on a specific domain (mathematics education). But we only tested our results on one domain. In this chapter, we expand our research into other domains.

We start by exploring the application of the distributed specificity algorithm that we introduced in chapter 4 to other technical domains. First, we quantitatively assess how well the distributed specificity performs in other domains. We will experimentally investigate if the minimal supervision observed in chapter 4 holds for other technical 
domains. We will also show the effect of increasing the amount of training data on the performance of our algorithms.

\subsection{Quantitative Experiments}

For a quantitative assessment of distributed specificity, as well as monitoring its performance with respect to the increase in supervision, we conduct a set of experiments on four new domains: microbiology, optometry, economics, and politics. Table 5.1 shows the corpora used for each domain for the experiments.

\begin{tabular}{|c|c|c|}
\hline Domain & Text Book & No. of Tokens \\
\hline Microbiology & Betsy and Keogh (2012) & 75,507 \\
Optometry & Elliott $(2013)$ & 189,756 \\
Economics & Reinert (2012) & 190,709 \\
Politics & Anderson (2005) & 129,042 \\
\hline
\end{tabular}

Table 5.1: This table puts forth the specifications of the corpora of the new domains

Since human annotation is labor-intensive and time-consuming, we use the terms detected by TermoStat (v.s., section 3.3.3) as ground truth for the quantitative cross domain evaluations carried out in this section. TermoStat receives each corpus as input, with no prior preprocessing, and outputs the detected terms. The rest of the vocabulary that has not been detected as terms is treated as non-terms. The number of non-terms far exceeds the number of terms in the vocabulary of the corpora we examined. To have a balanced dataset, for each corpus, we randomly sampled from the non-terms as many times as the size of the set of terms for that corpus. Distributed specificity receives an equal number of terms and non-terms for training, as well as for testing. It builds the local and global vectors, by the method described in section 
4.4.1 of this dissertation, for all the terms and non-terms. The local vectors are built using the CBOW architecture (v.s., section 2.4.4), with the window size of 5 , and a minimum frequency of 5 . The distributed specificity vectors of the training set are then passed on to a classifier for training. We train and compare a number of different classifiers. We then test our classifiers on a much larger test set. The statistics of the dataset built for each corpus is shown in table 5.2 below. The only preprocessing used for these datasets is a minimum frequency of 5. That is, any lexical unit (i.e., word type) with a frequency less than 5 is filtered out due to the parameters set for our local word embedding architecture.

\begin{tabular}{|c|c|c|c|}
\hline Domain & Terms & Non-terms & Dataset Size \\
\hline Microbiology & 479 & 479 & 958 \\
Optometry & 672 & 672 & 1344 \\
Economics & 440 & 440 & 880 \\
Politics & 316 & 316 & 632 \\
\hline
\end{tabular}

Table 5.2: The table shows the number of terms, non-terms, and total datapoints in our balanced datasets for the new domains

The mentioned in table 5.2 were divided into training and test sets as displayed below.

\begin{tabular}{|c|c|c|c|}
\hline Domain & Training Set Size & Test Set Size & Dataset Size \\
\hline Microbiology & 100 & 858 & 958 \\
Optometry & 100 & 1244 & 1344 \\
Economics & 100 & 780 & 880 \\
Politics & 100 & 532 & 632 \\
\hline
\end{tabular}

Table 5.3: The table shows the training and test set splits in our balanced datasets for the new domains

The training and test sets displayed in table 5.3 contain an equal number of terms and non-terms. 
We do not use the entire training set for all our experiments. We start with only 10 datapoints, that are randomly selected from the term and non-term lists, and will grow the training size up to 100 . We will use our highest performing classifiers from section 4.6, namely, Logistic Classifier, Multi-Layer Perceptron (MLP), and Support Vector Machines $\left(\mathrm{SVM}^{1}\right)$. The results of our method applied to the microbiology dataset are shown below.

\begin{tabular}{|c|c|c|c|c||c|}
\hline Classifier & $n_{\text {train }}=10$ & $n_{\text {train }}=20$ & $n_{\text {train }}=50$ & $n_{\text {train }}=100$ & average \\
\hline Logistic & 0.72 & 0.64 & 0.73 & 0.71 & 0.70 \\
MLP & 0.69 & 0.71 & 0.69 & 0.72 & 0.71 \\
SVM & 0.72 & 0.58 & 0.69 & 0.70 & 0.67 \\
\hline
\end{tabular}

Table 5.4: The table shows the performance of the distributed specificity algorithm in F1-score on the microbiology domain. $n_{\text {train }}=k$ signifies the training set size of $k$.

Table 5.5 below shows the performance of the distributed specificity algorithm in the optometry domain.

\begin{tabular}{|c|c|c|c|c||c|}
\hline Classifier & $n_{\text {train }}=10$ & $n_{\text {train }}=20$ & $n_{\text {train }}=50$ & $n_{\text {train }}=100$ & average \\
\hline Logistic & 0.71 & 0.65 & 0.66 & 0.70 & 0.68 \\
MLP & 0.56 & 0.56 & 0.73 & 0.72 & 0.64 \\
SVM & 0.71 & 0.65 & 0.66 & 0.67 & 0.67 \\
\hline
\end{tabular}

Table 5.5: The table shows the performance of the distributed specificity algorithm in F1-score on the optometry domain. $n_{\text {train }}=k$ signifies the training set size of $k$.

Table 5.6 below shows the performance of the distributed specificity algorithm in the economics domain.

Table 5.7 below shows the performance of the distributed specificity algorithm in the politics domain.

\footnotetext{
${ }^{1}$ We will use the scikit-learn implementation of SVM throughout this chapter instead of the Weka implementation in chapter 4
} 


\begin{tabular}{|c|c|c|c|c||c|}
\hline Classifier & $n_{\text {train }}=10$ & average $n_{\text {train }}=20$ & $n_{\text {train }}=50$ & $n_{\text {train }}=100$ & \\
\hline Logistic & 0.66 & 0.43 & 0.59 & 0.70 & 0.59 \\
MLP & 0.50 & 0.55 & 0.65 & 0.66 & 0.59 \\
SVM & 0.44 & 0.46 & 0.60 & 0.68 & 0.59 \\
\hline
\end{tabular}

Table 5.6: The table shows the performance of the distributed specificity algorithm in F1-score on the economics domain. $n_{\text {train }}=k$ signifies the training set size of $k$.

\begin{tabular}{|c|c|c|c|c||c|}
\hline Classifier & $n_{\text {train }}=10$ & $n_{\text {train }}=20$ & $n_{\text {train }}=50$ & $n_{\text {train }}=100$ & average \\
\hline Logistic & 0.74 & 0.59 & 0.69 & 0.67 & 0.66 \\
MLP & 0.46 & 0.71 & 0.58 & 0.67 & 0.61 \\
SVM & 0.52 & 0.68 & 0.62 & 0.59 & 0.61 \\
\hline
\end{tabular}

Table 5.7: The table shows the performance of the distributed specificity algorithm in F1-score on the politics domain. $n_{\text {train }}=k$ signifies the training set size of $k$.

We attribute the variance in the F1-score of the tables above, within a classifier, to the quality of terms randomly selected in the training set, and not necessarily the growth in the size of the training data, hence the high performance observed where $n_{\text {train }}=10$. The results confirm the observations in our earlier studies indicating the superior performance of the distributed specificity equipped with a logistic classifier in minimal training (see table 4.1).

\subsection{Qualitative Assessments}

This section will present a qualitative assessment of the distributed specificity algorithm on the new domains (v.s. table 5.1). In the experiments below we use the logistic variant of the distributed specificity algorithm (v.s. section 4.4). The main reason for that choice is its highest resilience towards minimal training.

For the results shown in this section, we use a training set of size only 10. For 
details in regard to the selection of these datapoints section 5.1 can be consulted. We present the distributed specificity classifications for terms and non-terms of the mentioned corpora. The table below shows the results for the microbiology dataset. A few instances of false positive and false negatives can be seen that were corrected by the distributed specificity algorithm. These results are not handpicked by any means and were directly taken from the top of the list of results in their original random order that they were fed to the model for prediction at inference time.

Table 5.9 below shows the results of the distributed specificity algorithm as compared with TermoStat for the optometry domain. Similar to the microbiology domain, distributed specificity, shows a high quality term extraction in the optometry domain.

Table 5.10 below compares a random selection of terms and decisions made by the distributed specificity algorithm versus TermoStat's. The issue that we noticed in this domain was the abundance of numbers (e.g., dates, years, and quantities of goods) in various formats, that distributed specificity tends to recognize as terms in this domain. This was interesting to observe. For instance, international contracts or conventions can in fact be distinguished from one another by their dates or other numbers that may follow them. Consequently, the vectors for those specific numbers will inevitably carry some of the semantics of those terms.

The results in the political domain as shown in table 5.11 look high quality as well. We did not notice the challenges we observed for the economics domain in the political domain. It is worthwhile to note, however, that some characteristics of these corpora can be due to the idiosyncrasies in the specific sub-domains that the selected textbooks capture.

In this section, we offered a qualitative assessment of the results of applying dis- 


\begin{tabular}{|c|c|c|}
\hline Term Candidate & TermoStat & Distributed Specificity \\
\hline escherichia & 1 & 1 \\
\hline pattern & 0 & 0 \\
\hline adenosine & 1 & 1 \\
\hline mosquito & 1 & 1 \\
\hline person & 1 & 0 \\
\hline enterobacter & 1 & 1 \\
\hline against & 0 & 0 \\
\hline spore & 1 & 1 \\
\hline rate & 1 & 1 \\
\hline infection & 1 & 1 \\
\hline 88 & 0 & 0 \\
\hline muscle & 1 & 0 \\
\hline size & 1 & 0 \\
\hline chemical & 1 & 1 \\
\hline transfer & 0 & 0 \\
\hline special & 0 & 0 \\
\hline osmosis & 1 & 1 \\
\hline $\operatorname{co} 2$ & 0 & 1 \\
\hline live & 0 & 0 \\
\hline iodine & 1 & 1 \\
\hline feces & 1 & 1 \\
\hline processing & 1 & 1 \\
\hline pathogens & 0 & 1 \\
\hline streptococcus & 1 & 1 \\
\hline lactic & 0 & 1 \\
\hline solution & 0 & 1 \\
\hline pinocytosis & 1 & 1 \\
\hline rrna & 1 & 1 \\
\hline dalton & 1 & 1 \\
\hline until & 0 & 0 \\
\hline
\end{tabular}

Table 5.8: The table shows the results of the distributed specificity algorithm compared with the TermoStat's in the microbiology domain. $n_{\text {train }}=k$ signifies the training set size of $k$. 1 indicates a marking as a "term" and 0 indicates marking as a "non-term". The full table can be found in appendixA, table A.1. 


\begin{tabular}{|c|c|c|}
\hline Term Candidate & TermoStat & Distributed Specificity \\
\hline this & 0 & 0 \\
\hline metamorphopsia & 1 & 1 \\
\hline pp & 0 & 0 \\
\hline pursuit & 1 & 0 \\
\hline canthus & 1 & 1 \\
\hline down & 0 & 1 \\
\hline result & 1 & 0 \\
\hline retina & 1 & 1 \\
\hline 2004 & 0 & 0 \\
\hline disadvantage & 1 & 0 \\
\hline database & 1 & 0 \\
\hline blink & 1 & 1 \\
\hline combination & 1 & 0 \\
\hline figure & 1 & 0 \\
\hline coefficient & 1 & 0 \\
\hline novice & 0 & 1 \\
\hline cs & 0 & 1 \\
\hline thereby & 0 & 0 \\
\hline bar & 1 & 0 \\
\hline j.d & 0 & 0 \\
\hline review & 1 & 0 \\
\hline discuss & 1 & 0 \\
\hline factor & 1 & 0 \\
\hline alignment & 1 & 1 \\
\hline 5.10 & 0 & 0 \\
\hline driving & 0 & 1 \\
\hline analysis & 1 & 0 \\
\hline inspect & 1 & 1 \\
\hline progression & 1 & 0 \\
\hline correcting & 0 & 1 \\
\hline
\end{tabular}

Table 5.9: The table shows the results of the distributed specificity algorithm compared with the TermoStat's in the optometry domain. $n_{\text {train }}=k$ signifies the training set size of $k .1$ indicates a marking as a "term" and 0 indicates marking as a "nonterm". The full table can be found in appendixA, table A.2. 


\begin{tabular}{|c|c||c|}
\hline Term Candidate & TermoStat & Distributed Specificity \\
\hline instance & 1 & 0 \\
yen & 1 & 1 \\
vreeland & 0 & 0 \\
possibilities & 0 & 0 \\
notice & 0 & 0 \\
component & 1 & 0 \\
elasticity & 1 & 0 \\
dramatically & 0 & 0 \\
becomes & 0 & 0 \\
1999 & 0 & 1 \\
variable & 1 & 0 \\
credibility & 1 & 0 \\
holding & 1 & 0 \\
benefit & 1 & 0 \\
czech & 0 & 0 \\
decline & 1 & 1 \\
inter-firm & 1 & 1 \\
did & 0 & 0 \\
export & 1 & 1 \\
devaluation & 1 & 1 \\
five & 0 & 0 \\
disagreement & 1 & 0 \\
institution & 1 & 0 \\
opposed & 0 & 0 \\
internalization & 1 & 0 \\
millennium & 0 & 0 \\
primary & 0 & 0 \\
restriction & 1 & 0 \\
developments & 0 & 0 \\
actually & 0 & 0 \\
\hline
\end{tabular}

Table 5.10: The table shows the results of the distributed specificity algorithm compared with the TermoStat's in the economics domain. $n_{\text {train }}=k$ signifies the training set size of $k$. 1 indicates a marking as a "term" and 0 indicates marking as a "nonterm". The full table can be found in appendixA, table A.3. 


\begin{tabular}{|c|c||c|}
\hline Term Candidate & TermoStat & Distributed Specificity \\
\hline fail & 0 & 0 \\
britain & 0 & 1 \\
ethos & 1 & 1 \\
balance & 1 & 1 \\
opportunities & 0 & 1 \\
result & 1 & 1 \\
slaughter & 1 & 0 \\
corruption & 0 & 1 \\
calculation & 1 & 1 \\
france & 0 & 1 \\
impossible & 0 & 0 \\
skill & 0 & 0 \\
allegedly & 0 & 0 \\
open & 0 & 0 \\
big & 0 & 0 \\
legislative & 0 & 1 \\
oil & 0 & 1 \\
scientific & 0 & 1 \\
electorate & 1 & 1 \\
latest & 0 & 0 \\
earth & 1 & 0 \\
react & 0 & 0 \\
complexity & 1 & 1 \\
thereby & 0 & 1 \\
considered & 0 & 0 \\
dependent & 0 & 1 \\
ozone & 1 & 0 \\
lives & 0 & 0 \\
demand & 1 & 1 \\
need & 1 & 0 \\
& 0 & 0 \\
\hline
\end{tabular}

Table 5.11: The table shows the results of the distributed specificity algorithm compared with the TermoStat's in the politics domain. $n_{\text {train }}=k$ signifies the training set size of $k .1$ indicates a marking as a "term" and and 0 indicates marking as a "non-term". The full table can be found in appendixA, table A.4. 
tributed specificity algorithm with minimal supervision to four new domains. That is, not only the number of the training instances were low (i.e., 10), but also these instances were prone to noise escalating from the automatic extraction by TermoStat, as opposed to chapter 4 where the training data resulted from human annotation. Since we use a high-precision method to select the positive instances (i.e., terms), they are not as much prone to noise as the negative instances which are selected randomly from the pool of lexical units that TermoStat did not recognize as terms. We believe that increasing the negative instances size for training is an inexpensive operation that could improve the results quality of the distributed specificity algorithm even further.

\subsection{Domain Adaptation for ATE}

In this section, we explore the possibility of transferring terminological knowledge from one technical domain to the other. This transfer is in the form of predicting a target embedding distribution given a source embedding space.

\subsubsection{Introduction}

Transfer learning consists of applying the knowledge learned for one task by an algorithm, to another task. For instance, algorithms trained to tell images of cats, dogs, and horses apart can be adopted to distinguish automobiles from pedestrians with some modifications.

Domain adaptation is a subclass of transfer learning where the task stays the same but the distribution of the datapoints in a target domain is different from that 
of the source domain (Kan et al., 2015). The present study explores the possibility of application of such algorithms to the task of terminology extraction.

Corpora of different domains (e.g., optometry vs. mathematics) are comprised of different distribution of words. They share some of their words, at least as surface symbolic forms. Some of these shared words hardly ever have different meanings (e.g., function words, stop words). Our experiments used these semantically stable words in different corpora as anchor points in order to learn mapping from one lexical distribution (source) to another (target). We call these specific lexical units that enable such transformations anchor words. It has been shown that the distribution of words and phrases in one language can be accurately mapped to another (Mikolov et al., 2013d); however, the mapping of lexical distributions among different domains within a language poses new challenges to be addressed. For instance, many of the technical terms in one technical domain merely do not exist in the other.

In our experiments, we observed that learning a mapping between distributed representations of stop words in our source and target domains can bring the distribution of other words common in both domains closer in vector space. This implies that at least some of the geometry of a domain can be inferred from another. Further details about the dataset, methodology, and results are provided next.

\subsubsection{Dataset}

The mathematics corpus created in chapter 3 is used as our target-domain corpus for the following experiments. As for the source-domain corpora we used the corpora put forth in table 5.2. 


\subsubsection{Methods}

For any of the source domains mentioned above, we used the shallow neural network by Mikolov et al. (2013b) with the CBOW architecture to capture the local statistics of words in that domain. The same was performed for the target mathematics domain. The resulting neural network models take any single symbolic representation as input and return a continuous vector containing dense word co-occurrence statistics. For any source-target domains pairs (e.g., politics-mathematics) we trained a separate feed-forward neural network to learn the word mapping between each pair of sourcetarget domains. We hypothesized that learning the mapping of a handful of stop words, shared between a source and a target domain, can bring other common words closer to each other in vector space. In distributional semantics, the position (more accurately, their cosine distance) of the words relative to each other reveals their role and meaning in the lexicon. Therefore, if we can predict the positions of unseen target-domain words from their source-domain counter parts we have approximated their semantics from another domain. As a result, a distributed specificity term extraction algorithm can be theoretically trained only in one domain but applied to other domains. The experiments below are the first attempt towards this goal in ATE.

\subsubsection{Experiments and Results}

For each source-target domain pairs we trained a neural network regressor on the stop words shared between each source and target domains. These networks, therefore, were trained to transform one domain into another. To measure if this transformation brings the words under investigation closer in the vector space, we measured the cosine 
distance of the source words from their target domain counter-parts, before and after transformation. The results show that the words representations did indeed become more similar to their target-domain counter-parts as a result of the transformations learned on only the stop words that act as our anchor points. The stop words are used only to learn the parameters of the transformer network. We tested the network on the new words common between the source and target corpora that were not previously observed. We used cosine distance to measure the proximity of the words in vector space. In our experiments, this value varies between 0 and 1 , where 0 means there is no information shared between a pair of words and 1 means they are identical words. The values shown in table 5.12 show the gain in the similarity of the pair after the transformation and not their similarity.

\begin{tabular}{|c|c|c|c|}
\hline Domain Pair & Distance Reduction & Anchors & Generics \\
\hline Microbiology-Mathematics & 0.17 & 98 & 979 \\
Optometry-Mathematics & 0.11 & 101 & 1,749 \\
Economics-Mathematics & 0.13 & 104 & 1,859 \\
Politics-Mathematics & 0.11 & 101 & 1,333 \\
\hline
\end{tabular}

Table 5.12: The table shows the reduction of (cosine) distance between words as a result of the transformation for each domain pair. Four source distributions are transformed to target distributions using anchor words. The first column shows the source-target pairs, the second shows the reduction in cosine distance between the two on average, after transforming the source closer to the target, the third column shows the number of anchor points used for training, and the last column shows the number of generic words shared between the two domains excluding the anchor points.

As shown in table 5.12 above, the words shared between the source and target domain exhibit greater similarity after the transformation. This shows that the transformation has been successful in rendering shared words between the corpora more similar to each other in representation. The table, however, does not state whether 
or not the words that are different in the two domains are rendered more similar to each other by the same extent. In order to assess dissimilar words, a new test set was created. The size of the test set for each domain pair is presented in the last column of table 5.13 below. We constructed this dataset as described next.

For each source and target domain pair, all the source domain words that do not occur in the target domain were selected, then paired with randomly selected words from the target domain that do not occur in the source domain. The results show that these words have been affected substantially less by the transformation as compared to their counterparts in table 5.12 above; that is, in the politics domain they are $690 \%$ less affected, in economics 290\% less affected, in microbiology $240 \%$ less affected, and in optometry $80 \%$ less affected. See table 5.13 below for further details.

\begin{tabular}{|c|c|c|c|}
\hline Domain Pair & Distance Reduction & Anchors & Random Pairs \\
\hline Microbiology-Mathematics & 0.05 & 98 & 879 \\
Optometry-Mathematics & 0.06 & 101 & 1,629 \\
Economics-Mathematics & 0.03 & 104 & 1,577 \\
Politics-Mathematics & 0.01 & 101 & 1,142 \\
\hline
\end{tabular}

Table 5.13: The table shows the reduction of (cosine) distance between words not shared by the domains as a result of the transformation for each domain pair. Four source distributions are examined to see to what extent unshared words grow similar by error in transformation. The first column shows the source-target pairs, the second shows the reduction in cosine distance between the two on average after transforming source closer to the target, the third column shows the number of anchor points used for training, and the last column shows the number of random word pairs not shared between the two domains.

Some of the unshared word pairs stated above are brought below in table 5.14 as examples. 


\begin{tabular}{|c|c|c|c|}
\hline Domain Pair & Pair I & Pair II & Pair III \\
\hline Microbiology-Mathematics & cytosol-contest & prefix-infinity & covalent-window \\
Optometry-Mathematics & obstruction-careers & pursuit-refinery & indentation-penalty \\
Economics-Mathematics & Chile-solstice & poorest-instantaneous & exporters-gallery \\
Politics-Mathematics & managed-subtends & Asian-arch & crises-helpful \\
\hline
\end{tabular}

Table 5.14: The table shows instances of word pairs unshared between the each pair of domains.

\subsection{Summary}

This chapter illustrated the robustness of the distributed specificity algorithm, specifically, its flexibility and feasibility in being applied to different domains. This chapter also illustrated that lexical distributions pertaining to these different domains can transformed to one another while maintaining some characteristics of the original distribution. This is a substantial initial step towards domain-independent distributed NLP. A new dataset representing four new corpora were created in order to perform the experiments discussed in this chapter.

We had shown in chapter 4 that distributed specificity can be trained with minimal supervision for the mathematics education domain when receiving proper signal. Section 5.1 of the present chapter, in addition, showed that distributed specificity can be both minimally, as well as weakly trained for this purpose. That is, it displays robust performance even when the signal it receives is weak and a result of a specificity algorithm, as opposed to gold standard. Section 5.1 also showed, quantitatively, that distributed specificity can be minimally and more importantly, automatically trained for four different new domains, while maintaining robust performance.

Section 5.2 showed the quality of the terms extracted by the distributed specificity algorithm. In many instances this algorithm, although only minimally and weakly 
trained, was able to overcome noise and silence, to detect correct terms not originally captured by TermoStat.

Section 5.3 investigated the mapping between different terminological domains. This can enable algorithms based on distributed representation to be domain indifferent. That is, only with a mapping between the domains, a machine learning algorithm can be applied to any new domain without the need for retraining or extensive tuning. Particularly, this section explored if the information contained in the technical components of a distributed specificity vector can be transferred to another domain. In a vector space, a pair of word vectors can be said to share more information as the cosine value of the angle between them moves away from 0 . Ideally, we would like words that are shared between a pair of technical domains to become more similar than those unshared. The experiments showed that the algorithm does indeed transfer information from one domain to another, while affecting dissimilar words considerably less. To achieve this, the algorithm leveraged a set of anchor words shared between the two technical domains as training data and was tested on other words shared between the two domains. We found it particularly interesting that the algorithm used a very limited number of instances but was still able to generalize well. This method, to the best of our knowledge, has been used for the first time in the domain of automatic terminology extraction. 


\section{Chapter 6}

\section{Distributed Bridging Reference}

\section{Resolution}

Many NLP systems treat definite noun phrases as anaphors. However, definite noun phrases are also commonly used to 'bridge' to previously mentioned discourse referents (which are called 'anchors'). This bridging relation is different from co-reference, nevertheless, it requires correct identification of which previously mentioned entity the definite noun phrase is associated with. The present chapter uses word embeddings as a distributional semantic representation to address the task of bridging reference resolution. Specifically, we build a pairwise model where each pair is comprised of a mapping of anaphor and anchor phrases with the highest similarity score. We test the model with an anchor-distance of 2 and 5 sentences, where the latter reaches a slightly higher performance. We built a bridging dataset on the letters portion of the Open American National Corpus to test the performance of our system. 


\subsection{Introduction}

Coreference resolution is the task of finding all the mentions of every entity in text or speech. Correct resolution enables Information Extraction (IE) systems to acquire further information about the entity under inquiry. Similarly, bridging reference resolution - also known as associative anaphora (Hawkins, 1978), inferrables (Prince, 1981), and 'accommodation with cross-referencing' (Heim, 1982) - aims to find covert links among mentions. For example, in the text I went to a restaurant last night; the waiter was nice, the definite noun phrase (NP) the waiter does not corefer with any previously mentioned entity. The correct interpretation of this text involves adding a new discourse referent corresponding to the waiter and bridging this waiter to the restaurant: we understand the waiter as something like 'the waiter there', where there is the restaurant mentioned in the previous sentence.

Bridging thus differs from coreference in several ways. First, it is triggered when the previous discourse does not entail the existence of an $N$ at the point at which the $N$ occurs (Heim, 1982). Second, because there is no antecedent for the $N$, a new discourse entity is established corresponding to the mentioned $N$ - this new entity is 'accommodated' (e.g., (Lewis, 1979), (Heim, 1982)). Third, this new entity 'bridges to' a previously mentioned entity (Clark, 1975)); in our example, the waiter bridges to the restaurant. A person who didn't know how to make this bridge would not properly understand the text, and would thus lose valuable information that the discourse provides. The same holds for IE systems. This is especially problematic in light of the observation that bridging is common in discourse. For example, Poesio and Vieira (1998) report that 18\% of the definites in their collection are classified as bridging definites, and Spenader (2002) reports that between 20-30 percent of 
definites are cases of bridging (depending on the annotator).

It is sometimes argued that a broader notion of bridging should be used (see also note 2). For example, there are appropriate uses of definite NPs the $N$ in discourses that do not establish the existence of an $N$ but which nevertheless allow the $N$ to corefer with a previously mentioned entity. For example, in A student came to my office this morning. The boy was really upset, the boy corefers with a student, even though the existence of a student is silent on whether this student is male or female. This is common in anaphoric relations (e.g., A student walked in. He was confused about this grade.). We will refer to such cases as 'co-referent bridging' - these are uses of an anaphoric phrase (e.g., she, the student) which corefer with a previous entity as well as add their presuppositions to the information that is known about the antecedent (see Beaver and Zeevat (2012) for discussion). In the case of he, the presupposition is that this individual is male; in the case of the boy, the presupposition is that this individual is a boy (see e.g., Heim and Kratzer (1998)).

Both coreferent and non-coreferent bridging resolution tasks benefit coreference resolution. Coreferent bridging does so by resolving nominal anaphora that do not contain the antecedent's head. For instance, the $C D$ can later be referred to as the album (Versley, 2007). A source of error when word association and distributional semantic techniques are used in coreference resolution, is mistaking a non-coreferent bridging reference for a coreferent one (Feuerbach et al., 2015). However, if noncoreferent bridging references are resolved, such confusion will no longer occur. And that is a contribution that non-coreferent bridging reference resolution can offer to a coreference resolution system.

Number (1) below is an example of coreference, (2) is a case of coreferent bridging 
reference, and (3) is an instance of non-coreferent bridging reference. In this chapter, we are solely concerned with cases like (3): non-coreferent bridging reference.

1. I talked to Cyrus yesterday. He was still upset.

2. A student came to my office this morning. The boy was confused about his grade.

3. We went to that restaurant last night; the waiter was really nice.

As observed in the last two examples above, the bridging anaphors (the boy and the waiter) appear to share some semantic similarity with their respective anchors (a student and that restaurant). Recent experimental evidence suggests that human participants have difficulty comprehending texts in which the anchor and anaphor are semantically dissimilar (Singh et al., 2016). Such considerations suggest that it might be useful to use methods based on lexical ontologies and/or distributional semantics and word associations to address the task. Naturally, these methods have been widely investigated in the literature (see section 6.2). Our method is based on the recent distributional semantic representation of word embeddings (Pennington et al., 2014).

Currently available English datasets for bridging are rather small. GNOME was created for discourse and semantic properties of discourse entities that play a role in their realization and interpretation (particularly salience) and includes 58 (mereological $^{1}$ ) bridging descriptions (Poesio et al., 2004). (Spenader, 2002) was developed for an investigation of the many uses of definites more generally (of which bridging is just one) and contains 130 bridging definites. ISNotes was created for addressing information status (Hou et al., 2013b; Markert et al., 2012) and contains 663 bridging

\footnotetext{
${ }^{1}$ In part-whole relationship
} 
noun phrases. Hence more data would be useful for experimenting with and better understanding of bridging.

There are also discrepancies in the literature concerning the correct characterization of bridging. For example, GNOME only covers mereological bridging references (Poesio et al., 2004) among all the potential semantic relations bridging anaphora may have with their anchors. On the other hand, although ISNotes is not restricted to any specific semantic relation, it recognizes indefinite noun phrases as bridging anaphora (Hou et al., 2013a). Under this assumption, 'a page' in the utterance below is a bridging anaphor.

4. I finally bought the book, but a page was missing.

The argument is that it is not just any page that the noun phrase 'a page' refers to but it's a page in the book that was mentioned. This deviates from the original bridging reference definition, under which bridging anaphora are treated as given by the speaker, leaving the hearer to infer the intended antecedent (Clark, 1975). Under such a view, bridging is inherently about definiteness (see also Heim (1982)); the classification in Spenader (2002) is faithful to the classical view, though it innovates in a number of ways (such as allowing for multiple anchors). We hope to make sense of these conflicting characterizations of bridging in future work. For example, it is unclear how much of 'bridging' is related to so-called 'quantifier domain restriction'. A sentence like every student failed is typically understood as 'every student in some suitably restricted domain failed', where the suitably restricted domain might be the students in some particular class (among many other possibilities that are contextually resolved). For relevant discussion, see Chierchia (1995), Asher and Lascarides (1998), Roberts (1998), Anvari (2014). In sum, we follow the definiteness require- 
ment for bridging anaphora as per original definitions mentioned above. We develop our own dataset that both respects the definiteness prerequisite (unlike ISNotes) and contains a diverse set of bridging types (unlike GNOME).

Natural language processing has indulged in a continuous rise of embeddings (Manning, 2015). These embeddings have arrived with a variety of tastes and flavors (Mnih and Kavukcuoglu, 2013; Mikolov et al., 2013c; Pennington et al., 2014) and have proven to be a good asset to the field to the extent of ruling out the longstanding HMMs in speech recognition (Bengio and Heigold, 2014) and outperforming the persistent bag-of-words in text classification and sentiment analysis (Le and Mikolov, 2014). Global Vectors for word representation (GloVe) has been successfully applied to the related information extraction task of named entity recognition and outplayed similar word embeddings on the task (Pennington et al., 2014); thus, it is plausibly deemed a suitable candidate for the current bridging reference resolution task.

This study presents a mention-mention pairwise model to tackle the task of bridging reference resolution. Anaphor-anchor pairs are passed through a part of speech filter, then turned into mappings of word pairs from the anchor-anaphor phrases. Next, the highest scoring pair is fed into the classifier for bridging reference resolution. The results are reported with different filtering strategies applied to the new dataset developed in this study. This study apply bridging reference resolution to the new domain of the developed dataset.

Section 6.2 discusses the relevant literature on bridging reference resolution. Section 6.3 presents the dataset used in the study and mentions some of its characteristics.Then, we will discuss the methods used in the current work, in section 6.4. We provide the results and discussion in section 6.5 , before we conclude this chapter in 
section 6.6.

\subsection{Related Work}

Previous works in coreference and bridging reference resolution have frequently employed measures of word associations, semantic and lexical relations, and distributional semantics in their algorithms, as early as 1990s. Poesio et al. (1997) and Vieira and Poesio (2000) used WordNet with poor results on resolving bridging references. Poesio et al. (1998) used vector space representation to address bridging reference resolution and tuned different parameters to achieve their system's best performance. They found that their vector space model did not improve on their results with WordNet, however, Poesio et al. (2002) by using patterns to determine semantic relations and mutual information to track strength of associations for their vector space model, achieved eye-catching improvement over the previous algorithms. Poesio et al. (2004) successfully integrated Google distance into their algorithm, where the distance is the reciprocal of the Google search engine's hits for the pattern applied to the anaphor and the potential anchor. Recently, Feuerbach et al. (2015) used features based on words and context expansion from a distributional thesaurus, and automatically mined IS-A relations improved on resolving coreferent bridging references, which in turn improved the performance of Berkeley Coreference Resolution System (Durrett and Klein, 2013). Versley (2007) combined methods drawing on high-precision resources (GermaNet and web-based pattern search) with similarity and association methods (ranked similarity lists) to resolve coreferent bridging, which in turn improved their overall recall for coreference resolution. They interestingly find that a distance-bounded semantic similarity method using most similar words in the 
previous discourse with a semantic class-based filter and a distance limit is almost as good in precision as using surface patterns (Versley, 2007).

Hou et al. (2013b) used Markov logic networks (Domingos and Lowd, 2009) for unrestricted $^{2}$ bridging reference resolution. This allows them to benefit from global constraints as well as local features. Hou et al. (2013b) also implemented a pairwise ${ }^{3}$ mention-entity model with the same local and global features. For the local features they extended and modified features from Poesio et al. (2004) and used semantic, salience, surface, and syntactic information. As for global features, they imposed discourse-level constraints to account for global salience, as well as frequent bridging relations. In addition to two different versions of their Markov logic networks, they used three versions of a pairwise mention-entity model which uses $S V M^{\text {light }}$ for classification. The difference among versions was mostly in their coverage of the local and global features. They tested their systems on ISNotes dataset using 10-fold cross validation.

Previous works on automatic bridging reference resolution distinguish between bridging anaphor recognition and antecedent selection (Vieira and Poesio, 2000; Rahman and Ng, 2012; Cahill and Riester, 2012; Markert et al., 2012; Hou et al., 2013a,b); the present work is concerned with the latter task.

\footnotetext{
${ }^{2}$ Unrestricted here means that there are less restrictions in defining a bridging anaphor (e.g., it can be even an indefinite noun phrase) and what its relation to the anchor is (e.g., it is not limited to mereological relations as in Poesio et al. (2004)).

${ }^{3}$ As discussed by Soon et al. (2001)
} 


\subsection{Data}

We created a new bridging dataset on the letters portion of Open American National Corpus (OANC). The OANC is comprised of contributed texts, written in American English no earlier than 1990. The corpus consists of 106 letters with a total of 38,000 words. The letters were all requests for donations or financial contributions to support a charity organization or post-secondary school fund. Therefore this is a different domain than ISNotes. On average, the letters were 359 words and contained 18 sentences each; thus, the sentences were typically about 20 words long. The dataset consists of 327 anaphors, 288 antecedents, and 317 false antecedents throughout the corpus, which works out to an average of approximately 3 bridging cases per letter. Of the 288 antecedents, 225 were nouns, 44 were adjectives, and 19 were verbs. This distribution is used as a guide for our filtering phase (see section 6.4). False antecedents were 144 nouns, 64 adjectives, 74 verbs, 7 adverbs and 28 other constructions. All of the anaphors were noun phrases. Table 6.2 shows the distribution of the semantic relations between bridging anaphors and their anchors.

\begin{tabular}{||c|c||}
\hline Syntactic Category & Frequency among Anchors \\
\hline \hline Nouns & 225 \\
Adjectives & 44 \\
Verbs & 19 \\
\hline
\end{tabular}

Table 6.1: Syntactic distribution of bridging anchors in our dataset

\begin{tabular}{||c|c|c||}
\hline Semantic Relation & $\#$ & $\%$ \\
\hline \hline Hypernymy/Hyponymy & 31 & $9 \%$ \\
Meronymy/Holonymy & 48 & $15 \%$ \\
Encyclopedic & 248 & $76 \%$ \\
\hline
\end{tabular}

Table 6.2: Anaphor-anchor lexical relations in our dataset 
We used two annotators; an undergraduate student in cognitive science (annotator 1) and a graduate student in cognitive science with an undergraduate degree in linguistics (annotator 2); both males and in their 20s. The annotators were instructed to mark any definite noun phrase for which a previous mention does not exist and its presence is mediated by another phrase (noun phrase, verb phrase, adjectival phrase, adverbial phrase, or other phrase). Annotators were instructed to mark an incorrect antecedent for each bridging anaphor which we have called false antecedents. The annotation has been mainly carried out by annotator 1. Annotator 2 annotated 10\% of the designated corpus for computing the agreement $\kappa=0.61$ for bridging anaphora and their anchors. Typically, bridging phenomena have had relatively low agreement scores as compared to other tasks (Poesio and Vieira, 1998; Markert et al., 2012). This can speak to the diversity and volatility involved in the bridging reference resolution task.

\subsection{Methodology}

The present work is a first attempt at resolving non-coreferent bridging references using a mention-mention pairwise model where the mentions are represented by their corresponding word embeddings. After obtaining the embedding representation of a pair, it is fed to the classifier to make the final decision. To build the pairwise model, the algorithm looks at the current and the previous $\mathrm{k}$ sentences, where $\mathrm{k}$ is set to 2 or 5 in our experiments. We chose these values because Hou et al. (2013b) pointed out that $81 \%$ of NP anchors occur in the same up to previous 5 sentences and $71 \%$ of them occur in the current up to 2 previous sentences. From these phrases and the anaphor phrase, we construct a positive and a few negative pairs. 
A positive pair comprises the anaphor phrase and the anchor phrase and a negative pair consists of the anaphor and non-bridging anchor phrases. These phrases are then passed through our part-of-speech (POS) filtering using a MaxEnt POS tagger from OpenNLP 1.6 (Apache Software Foundation, 2014). We use three different methods for filtering. These filters are based on the distribution of bridging heads, that is nouns, adjectives ${ }^{4}$, and verbs. Penn Treebank POS tags (Marcus et al., 1994) are used in all of the filters.

Our three filters are the following:

- NNVBJJ: Filter out everything but nouns, verbs, and adjectives

- NNVB: Filter out everything but nouns, and verbs

- NN: Filter out everything but nouns

In the filters above, we have a separate filter for nouns and verbs only, since bridging anaphora are noun phrases and nouns, and some high-frequency adjectives (such as good, great and many alike) do not bear useful information for the task even if they are highly associated with the anaphor phrase's head. In general, we have higher expectations for the NN filter, as nouns constitute an overwhelming majority of the bridging reference anchors both in our data (see section 6.3) and other datasets (Hou et al., 2013b). After the filtering phase, we create mappings of words (anaphorWord:anchorWord) from the remainders of the anaphor and anchor phrases. These mappings are then converted to mappings of their corresponding word embeddings. We employ the 6 billion token variant of GloVe (Pennington et al., 2014), built on Wikipedia 2014 and Gigaword 5 (Parker et al., 2011). This variant is

\footnotetext{
${ }^{4}$ We exclude comparative and superlative adjectives since they do not occur as anchors in our dataset
} 
uncased with a vocabulary size of $400 \mathrm{~K}$. We use 50 dimension vectors for the task at hand.

Finally, the acquired embedding mappings are fed to the $\mathrm{SVM}^{5}$ classifier that uses the $\mathrm{SMO}^{6}$ algorithm for training (Hall et al., 2009). SVM has been previously employed for the bridging reference resolution task by Hou et al. (2013b).

\subsection{Experiments \& Results}

We use 10-fold cross validation throughout our experiments. Our method called embedding maps is tested with the different filters and anchor distances described in section 6.4. To find the best value for $k$ and the best type of filter we ran our method on ISNotes first. It was observed that the best performing method is our embedding maps $2 N N V B$.

To test the performance of our system, it was ran against our new dataset (see section 6.3 for more information on this dataset). This dataset is almost balanced. In the dataset, a few anaphors may have more than one correct anchor in the dataset, but on the other hand, some correct anchors may have more than one anaphor, which trivially increases the size of the negative data points, comparatively, in the dataset. We applied our best performing system (embedding maps $5 \mathrm{NN} \mathrm{VB}$ ) to this dataset and obtained the results presented in table 6.3. Our method shows good performance in this domain (76.55\%). We attribute this performance (at least partially) to the adequate distributional semantic representation carried out by the word embeddings.

The baseline method picks the majority class in the dataset. This accounts for

\footnotetext{
${ }^{5}$ Support Vector Machines

${ }^{6}$ sequential minimal optimization
} 


\begin{tabular}{||c|c|c||}
\hline Algorithm & F-Measure & Accuracy \\
\hline \hline Baseline & 0.374 & $53.62 \%$ \\
EM & $\mathbf{0 . 7 6 4}$ & $\mathbf{7 6 . 5 5 \%}$ \\
\hline
\end{tabular}

Table 6.3: Results of our method (embedding maps) in comparison to the majority class baseline on the dataset

roughly $54 \%$ of the correct labels. It is worth noting how unforgiving F-Measure is as a metric to such a baseline.

\subsection{Summary}

The present chapter shows a first attempt towards using distributed representation for the task of bridging reference resolution. We offered a dataset that not only complies with the original definition of the bridging phenomenon but also offers a diverse set of bridging relations. We developed a simple model based on word embeddings and part of speech tagging that performs much higher than a majority class baseline for the created dataset. 


\section{Chapter 7}

\section{Conclusion and Future Research}

\subsection{Summary}

This work introduced the application of distributed representation of words to the domain of information extraction, more specifically, two tasks of automatic terminology extraction and bridging reference resolution for the first time.

I offered a gold standard to evaluate ATE algorithms on the mathematics education domain. The dataset was developed using human annotators and the performance of three terminology extraction tools on a corpus of school mathematics textbooks was investigated. We used a post hoc evaluation mechanism using a tool called Term Evaluator which sped up the creation of the dataset. The dataset benchmarks three ATE methods which facilitates comparisons in future studies. The employment of Term Evaluator eliminated the need to annotate 1344 of the 4011 words, representing $33 \%$ of the terms extracted by TermoStat, which resulted in a significant saving in evaluation time. The results suggest that of the three tools examined, TermoStat, with stable high-precision and recall scores, is the most suitable tool for technical 
term extraction in a corpus of mathematics textbooks, validating the efficiency of its patterns and statistical tests. The apparent lower performance of TermoStat for the single-word category may have been caused by some term extraction and annotation related issues. For instance, words such as 'two' or 'three' were extracted by the other tools and marked as correct terms by annotators, but TermoStat regards these words general-domain terms. Another issue is some inconsistency in annotation that can be prevented by the Term Evaluator's memory, if used. There were terms marked as incorrect for TermoStat and correct for the other tools by the annotators (e.g., calculator, speed). A further issue may have arisen due to lack of efficient preprocessing in Topia and AntConc. AntConc and Topia extract terms like "zeros" as technical, whereas TermoStat does not. That is, AntConc and Topia do not recognize inflection, which in turn results in candidate terms such as "zero" and "zeros" both being evaluated as correct by the annotators. TermoStat, on the other hand, benefits from proper preprocessing and recognizes "zeros" as an inflected form of "zero. This can make TermoStat's recall seem lower than it actually is. Finally, it is worthwhile noting that various single-word mathematics terms (e.g., addition, number, and calculator) may be hard to judge as technical or not, especially since these terms are frequently used in general English.

This work then offered two implementations of a novel ATE approach called distributed specificity. The first uses the distributed representation of words as a filter for the task of unigram term extraction. To do so, we leveraged the local and global embeddings to represent a term, its senses, and its distributional characteristics. We used the global word embeddings GloVe pre-trained on general corpora, then we used the skip-gram and CBOW architectures to train local vectors on our technical do- 
main corpus. This was done in order to preserve both the domain-specific and the general-domain information a word may possess, including its syntactic and semantic behavior. These local and global vectors were concatenated into a single specificity vector to be fed to the classifiers. We showed that such a filter, with only as few training instances as 9, can substantially improve the output of the three ATE tools in unigram term extraction. This indicates that with a) any high-precision (even with very low recall and F-measure) term extraction tool that outputs a few terms, b) a few random generic words in a language, and c) our filter, one can create a highperformance term extraction system for that language. Our method can also be used as a way to combine different tools to benefit from the advantages that each can offer, resulting in a gain in performance. The use of the filter is not limited to combining term extraction tools; rather, it can be applied as feasibly to any individual term extraction method alone. It is important to note that in our study the improvement in performance is not due to the merger of different tools but to a richer, more elaborate, and more informative representation of the candidate terms. It was observed that the two local architectures, CBOW and skip-gram, do not show a considerable difference in capturing the technical sense and behavior of a word for unigram term extraction for the filtering approach.

In our second approach, we applied the classifiers trained on only 6 positive and 18 negative instances directly to the corpus. The CBOW local architecture performed consistently higher than skip-gram. SVM was our best performing classifier. This method currently falls 0.05 F-measure points behind TermoStat (partially due to the fact that it is not favored by the dataset as not all its results are not part of the annotation), yet it emphasizes that using distributed specificity, ATE classifiers can 
be successfully trained with very little training data, just as observed with our filtering approach. This fact encourages new minimally supervised ATE algorithms to appear in the future. In order for it to be able to compete with the filtering method, this approach needs to overcome the imbalanced nature of the dataset.

Our novel approach, distributed specificity, not only substantially outperformed previous methods, but it also eliminated the need for feature engineering and feature extraction altogether for ATE. In other words, it is no longer required to carefully design language dependent features in order to achieve higher quality term extraction. In contrast to other supervised methods, distributed specificity is minimally supervised such that the training instances can be acquired fully automatically by the combination of a statistical high-precision method (for positive instances) and random selection of generic words in a language (for negative instances). Both of these operations are low cost and readily available.

We applied our method to four different new domains: microbiology, optometry, economics, and politics. We evaluated the method quantitatively on minimal training data automatically acquired by a high-precision method and random selection of generic vocabulary items. The results showed a high F1-score on all these domains; this is a promising result, since is often challenging to obtain high performance for any domain.

In addition, we assessed the quality of the terms acquired by our algorithm for these four new domains. Although the algorithm is both minimally and weakly trained it is able to correctly classify many instances mis-classified by TermoStat. This is especially worth noting since the algorithm receives its signals from TermoStat itself.

It has been shown previously in NLP that words can be mapped across languages 
using distributed representation. It is a substantial finding since algorithms can be trained in one language and readily applied to others after a language transformation. However, this was not the case across domains. The reason is a one to one mapping does not exist among different terminological domains. That is, different domains have different terms and often these terms do not even occur in other terminological domains. However, the problem of ATE is that of binary classification and can be addressed across domains theoretically in two steps.

- Step 1. Constructing a mapping between the generic words common among the two domains

- Step 2. Transforming the technical terms from the source domain in relation to the transformed generic terms

In this dissertation, we took on the first step of the two above. We showed that common words can be mapped onto their counterparts in another domain so that this mapping would result in a considerably higher cosine similarity between similar words from the two distributions as compared with dissimilar words randomly selected from the words not shared between the two lexical distributions. This is a successful initial step towards domain adaptation in ATE. It is worth noting that we intentionally avoided the choice of a cosine based cost function to train this transformation, since we intended to assess if the geometry of the generic words would be preserved from one domain to another. We did observe that the cosine similarity of the similar words improved, albeit our error was based on the euclidean distance. This provides evidence for the first time that generic words preserve their geometry across domains.

Finally, we investigated the capacity of a method based on word embeddings to handle the bridging reference resolution. Our method uses mappings of embeddings 
to represent anaphor and anchor phrases and three POS filters. We created a new bridging reference dataset on the letters portion of the Open American National Corpus that abides with the original definition of bridging reference (see section 6.1 for further details) and tested our method once more. We obtained the accuracy of $76.55 \%$, that is noticeably higher than the baseline of $53.62 \%$.

\subsection{Future Work}

We have shown the success of our method for unigram term extraction across domains. We have not, however, tackled the problem of multi-word term, mainly since TermoStat displayed a higher performance on the multi-word terms. It has been shown that deep learning architectures, specifically CNNs and RNNs, are effective methods for identifying multi-word expressions (Gharbieh et al., 2017). Multi-word expressions cover a wide variety of linguistic phenomena that may include terms. In the future we would like to combine the methods from Gharbieh et al. (2017) with our distributed specificity algorithm to extract multi-word technical terms. It would be worthwhile to investigate if skip-gram and CBOW architectures can diverge in performance in extraction of terms that contain more than one word.

Polysynthetic languages have a high morpheme-to-word ratio; that is, most of the grammatical and semantic information of a sentence is carried inside individual words, but continuous distributed models, including our distributed specificity, predominantly disregard word-internal structures. Employing a recent method based on the skip-gram architecture that captures subword information in its word vectors (Bojanowski et al., 2016), we plan to address polysynthetic languages as a future step. Character embeddings can be combined with RNNs as another viable approach to 
perform ATE on polysynthetic languages that we plan to address in our future studies. We would also like to apply our novel methods to other Indo-European languages including French and Spanish, in future research.

Term extraction is an integral part of a variety of downstream NLP tasks. We plan to employ our methods to improve modern information retrieval, machine translation, and summarization. These systems nowadays are all based on distributed representations, which distributed specificity can feasibly merge with.

Finally, our experiments indicate that information can be shared between technical domains; however, we have not shown if this transferred information can help a term extraction algorithm benefit from other domains. In particular, the second step mentioned above, transforming the technical terms from the source domain in relation to the transformed generic terms, needs to be studied. This can result in an ATE algorithm being trained in only one domain but being readily applied to any other domains and languages. We intend to explore this avenue in future research.

In addition, we have not utilized any explicit mechanism to prohibit dissimilar words from becoming more similar (even if by a negligible amount) as a result of the transformations carried out by the algorithm; explicit cost functions can be modified to serve this purpose. This is another topic that can be explored in future research.

As for bridging reference resolution, we intend to include all of the words of the phrases in our representation by evaluating the following methods to achieve the most suitable representation:

1. Convolutional neural nets

2. Recurrent neural networks 
3. Fixed-sized ordinarily forgetting for variable length embeddings (Zhang et al., 2015)

The approaches mentioned above will replace our naive filtering mechanism. For this purpose, the effects of the methods for merging embeddings need to be investigated for the bridging reference resolution task. Using other vector space models, including holographic memories (Jones and Mewhort, 2007), the VSM offered by (Mikolov et al., 2013c) and a combination of them for bridging reference resolution is another possible direction of future research. It was noted that there is a discrepancy in the literature with regard to the characterization of bridging. In a forthcoming study, we will attempt to shed further light on the conflicting characterizations of the bridging phenomenon (see section 6.1 for further information). 


\section{Appendices}




\section{Appendix A}

\section{Full List of Results in Qualitative}

\section{Assessments}

This appendix enlists all the term candidates classified by the distributed specificity

algorithm compared with that of TermoStat's, in all of the 4 new domains as described in section 5.2 .

\section{A.1 Microbiology}

\begin{tabular}{|l|l|l|}
\hline Term Candi- & TermoStat & Distributed \\
date & & Specificity \\
\hline escherichia & 1 & 1 \\
pattern & 0 & 0 \\
adenosine & 1 & 1 \\
mosquito & 1 & 1 \\
person & 1 & 0 \\
enterobacter & 1 & 1 \\
against & 0 & 0 \\
spore & 1 & 1 \\
rate & 1 & 1 \\
infection & 1 & 1 \\
88 & 0 & 0 \\
muscle & 1 & 0
\end{tabular}




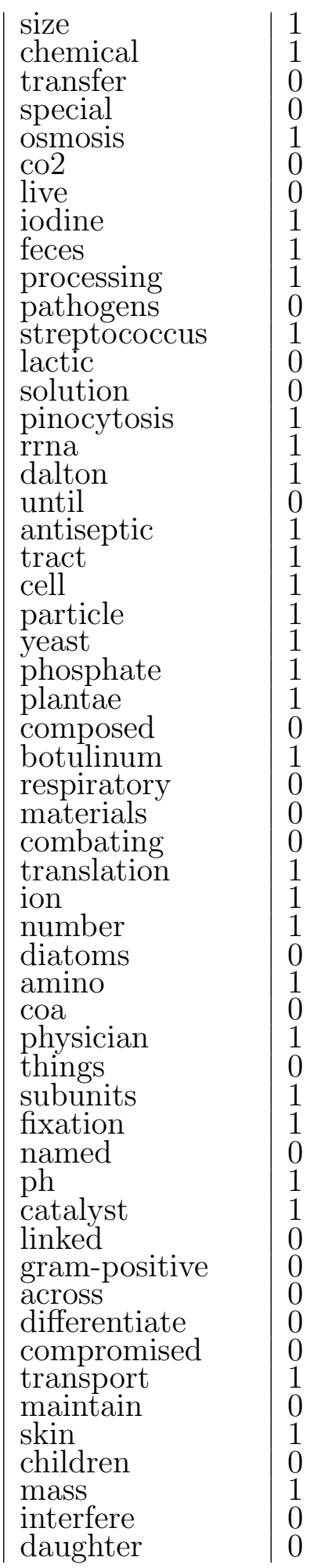

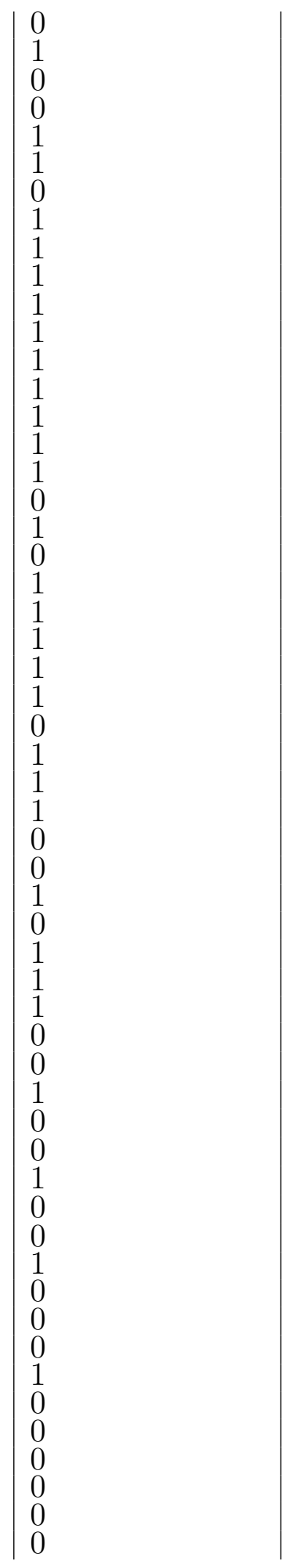




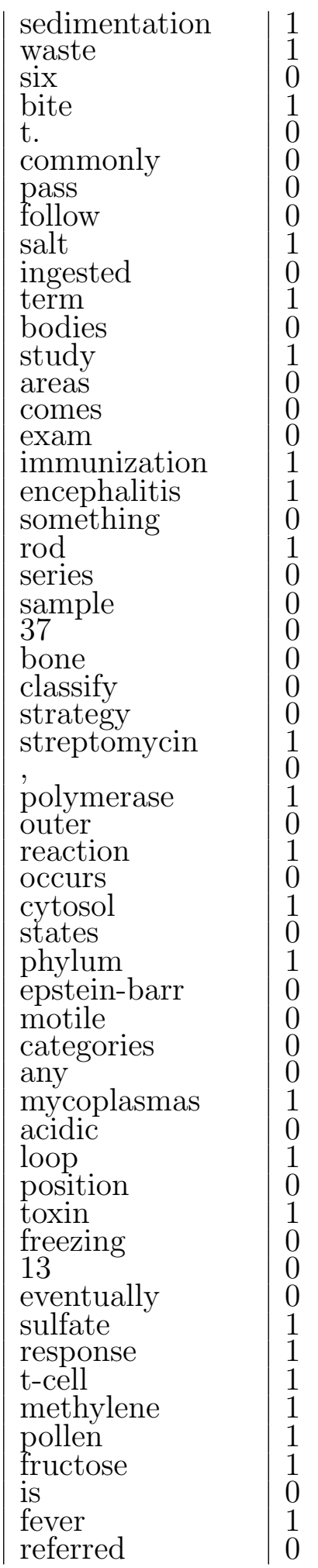
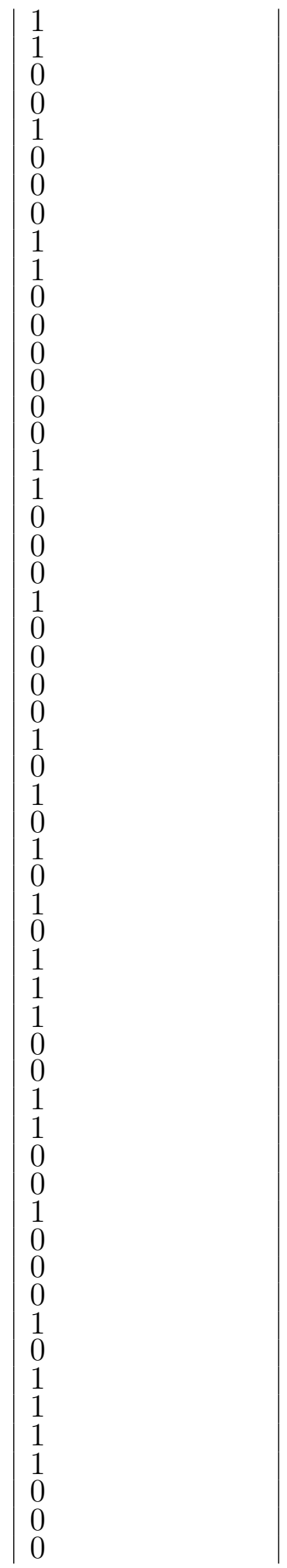


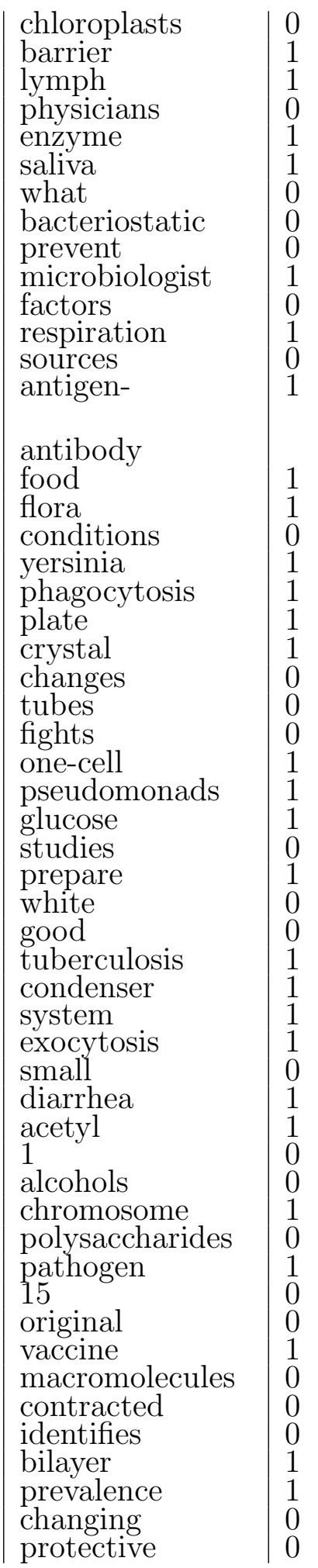

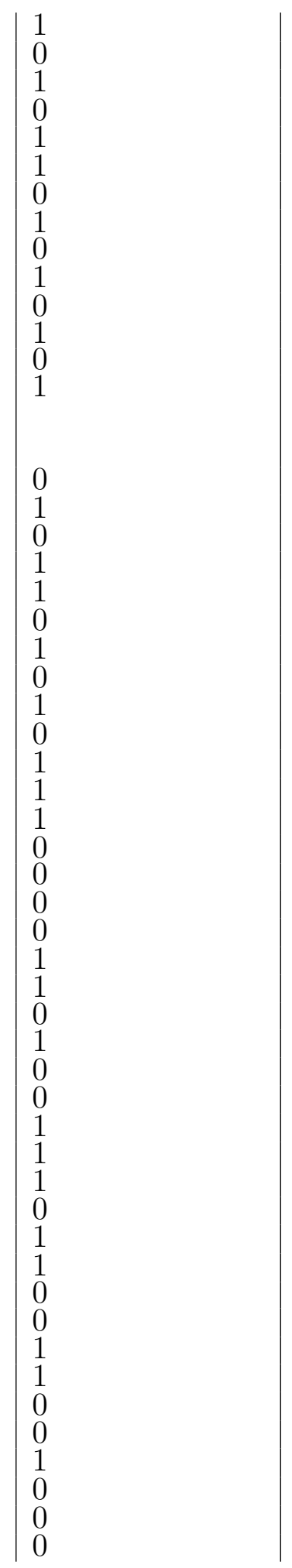




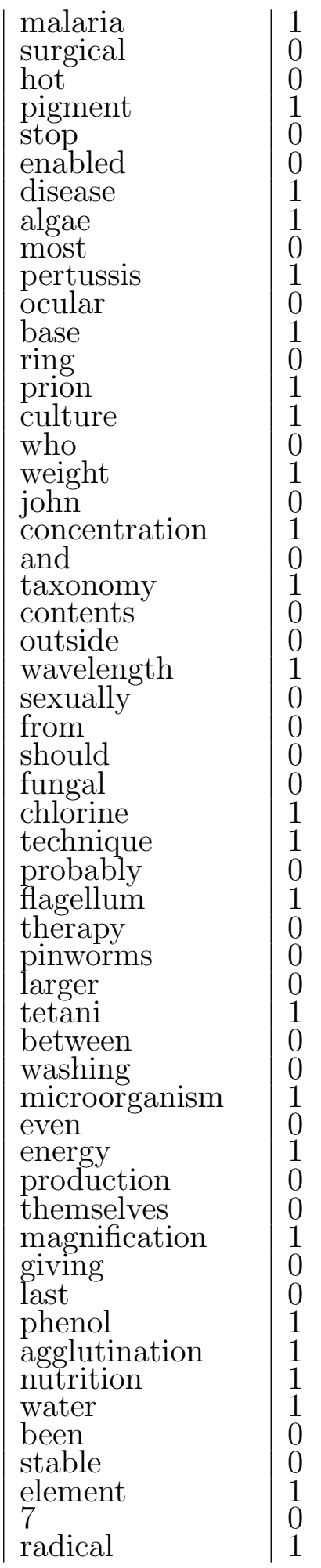

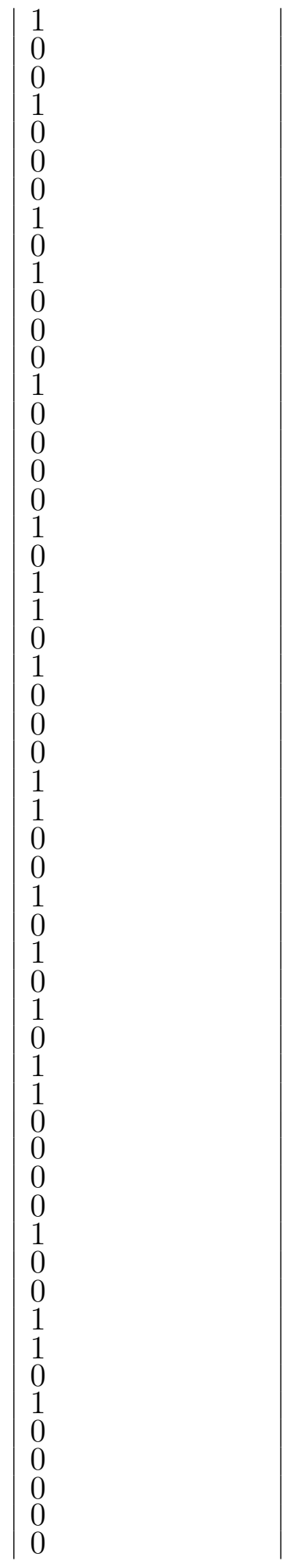




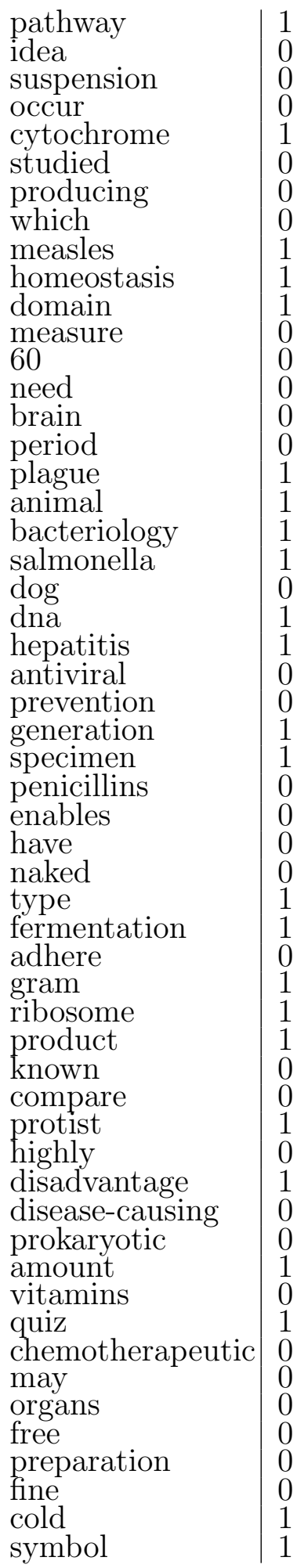

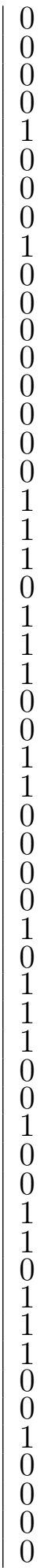




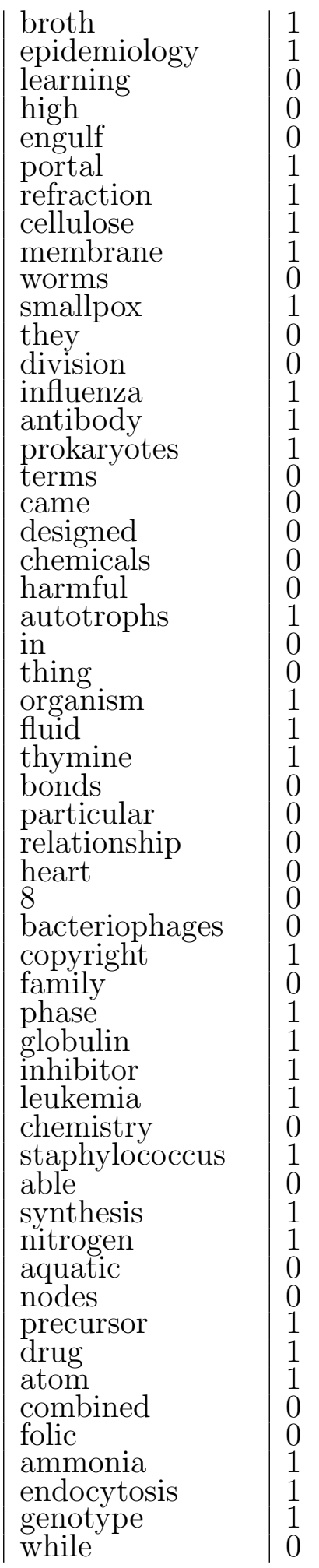

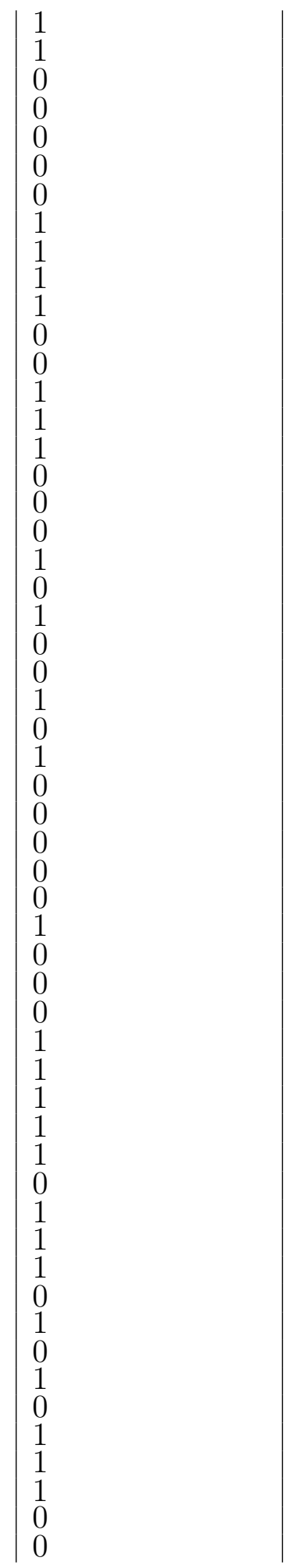




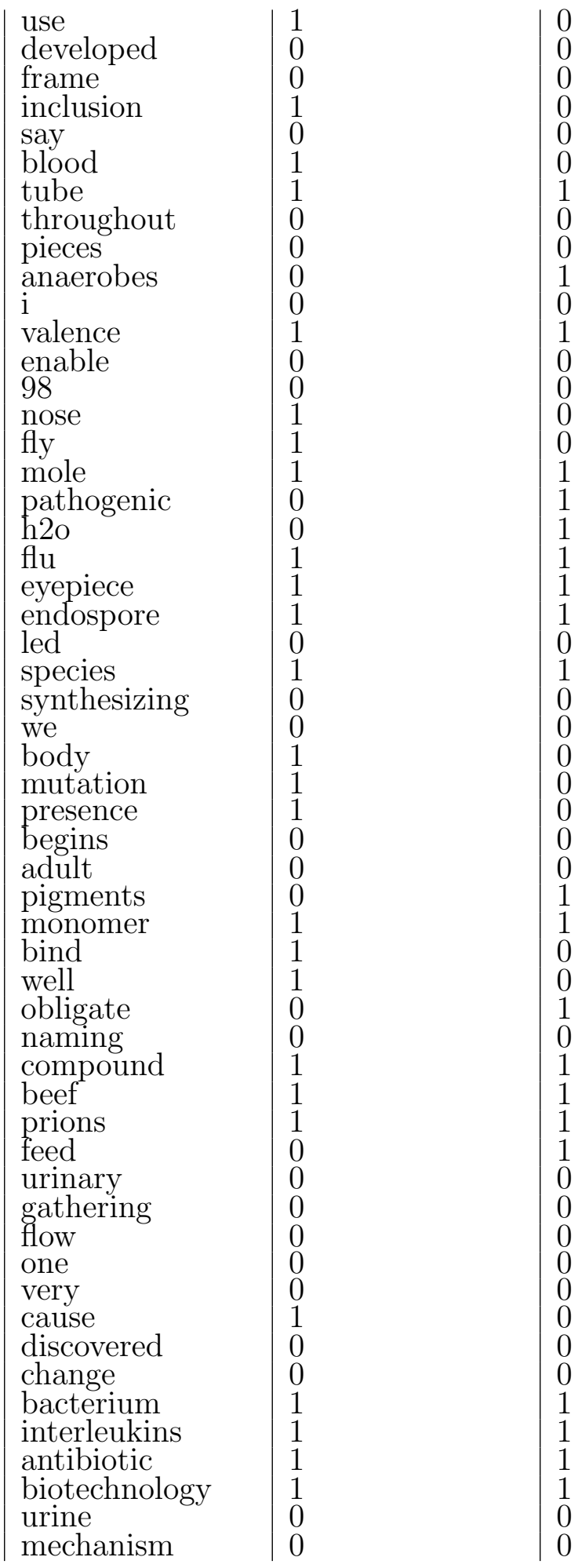




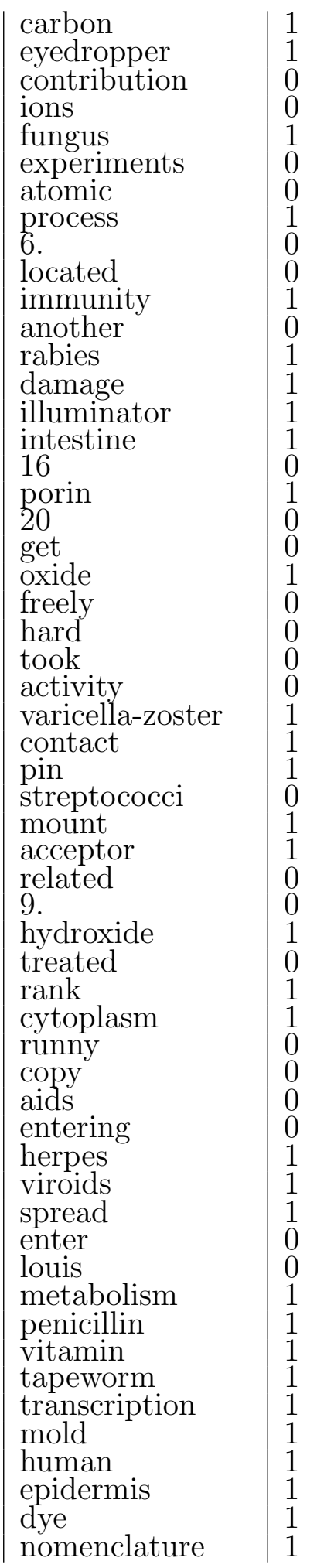

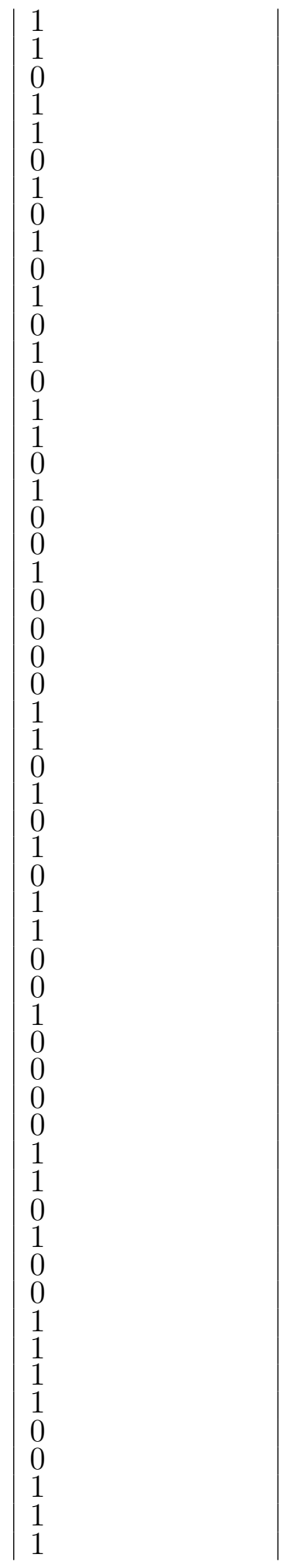




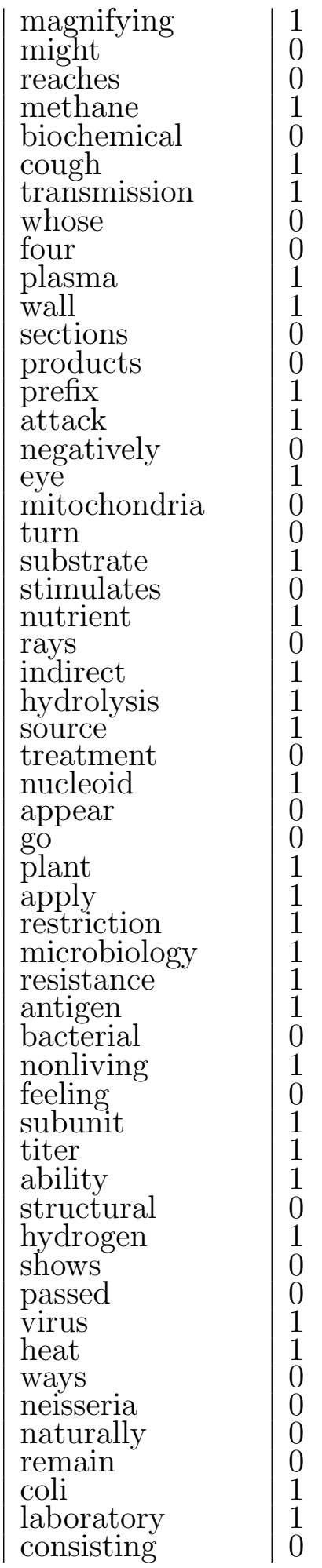

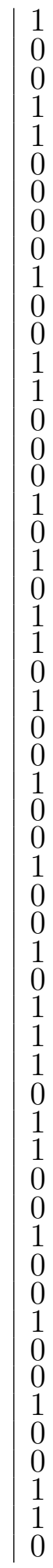




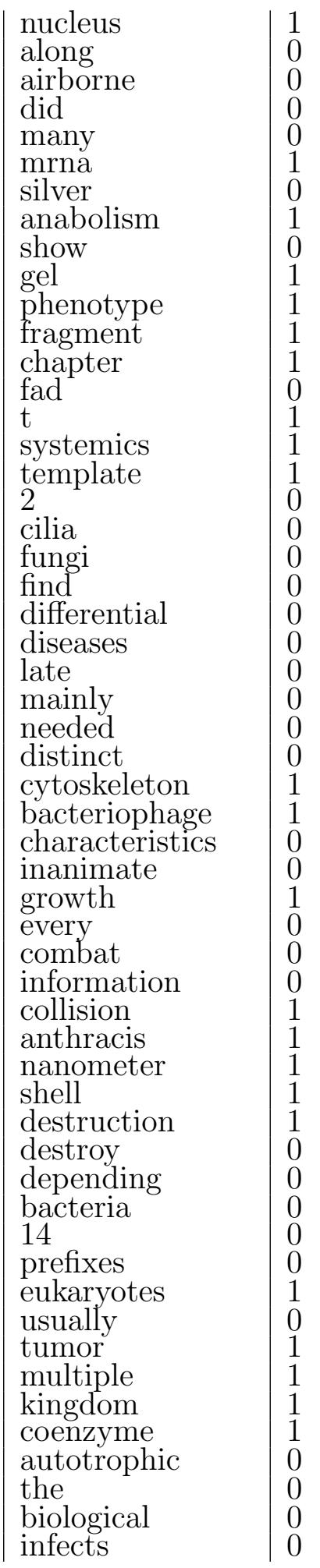

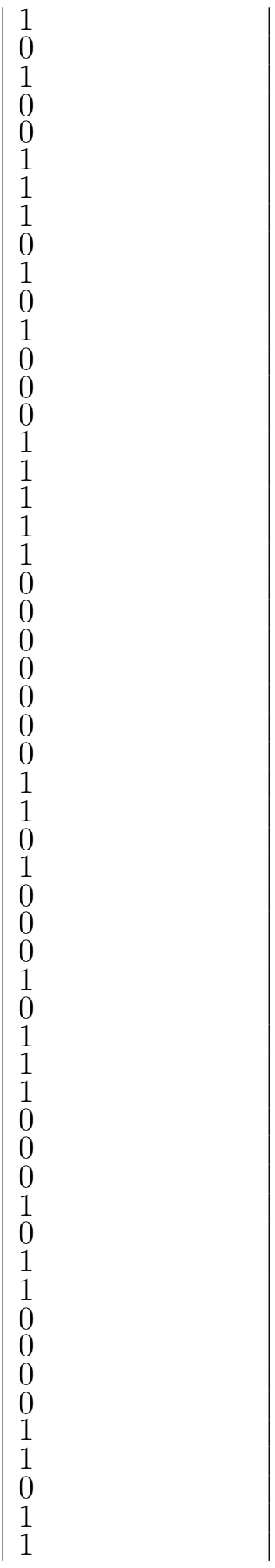




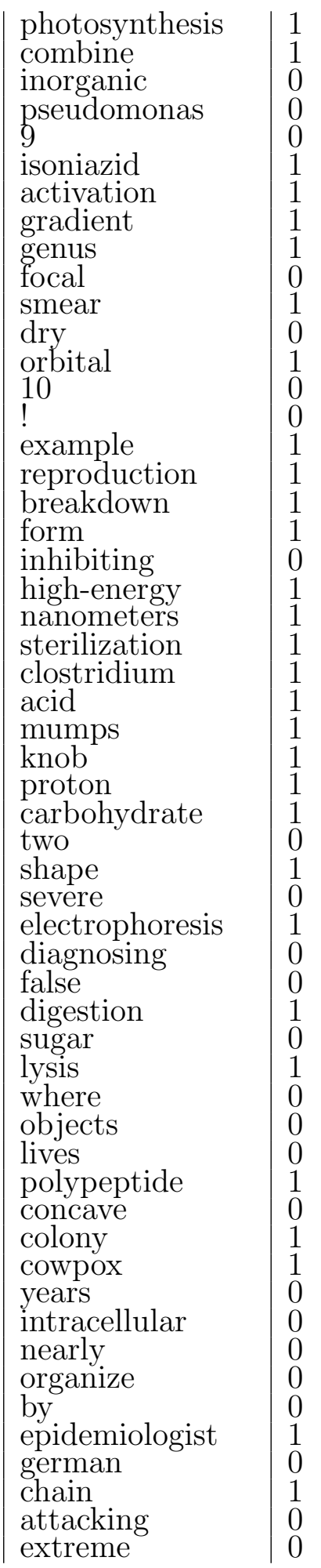

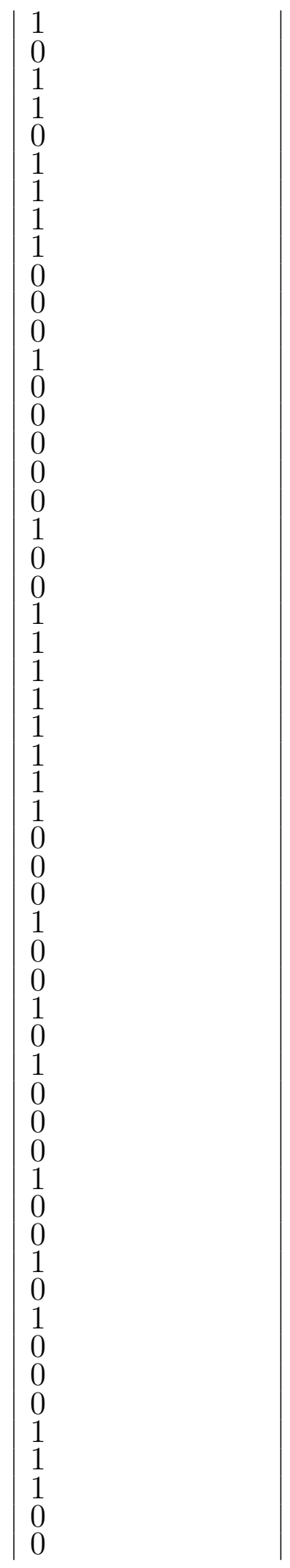




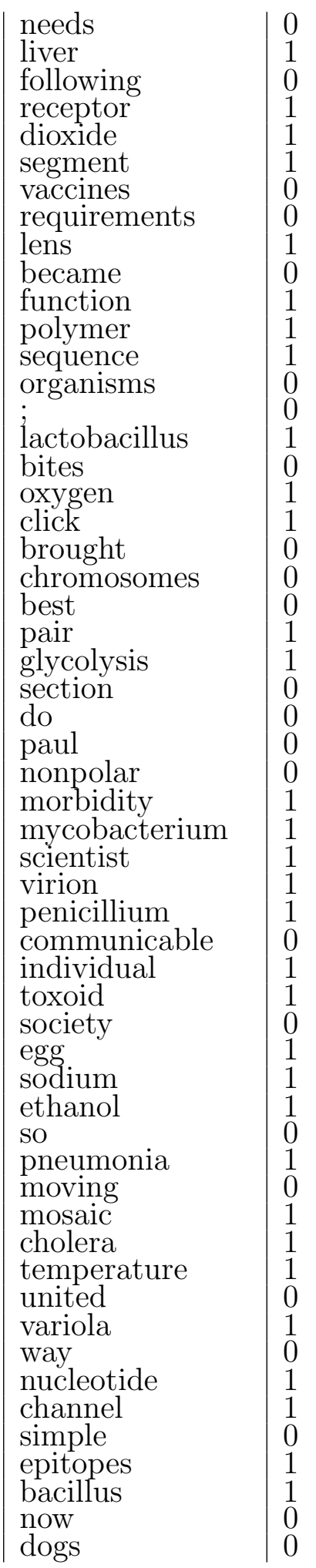

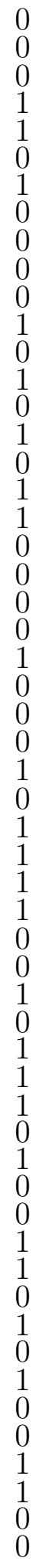




\begin{tabular}{|c|c|c|}
\hline glass & 1 & 0 \\
\hline measurement & 1 & 0 \\
\hline alcohol & 1 & 0 \\
\hline trna & 1 & 1 \\
\hline needle & 1 & 1 \\
\hline affect & 0 & 0 \\
\hline wave & 1 & 0 \\
\hline strain & 1 & 0 \\
\hline phagocytes & 0 & 1 \\
\hline carbohydrates & 0 & 1 \\
\hline vancomycin & 0 & 1 \\
\hline latin & 0 & 0 \\
\hline occurred & 0 & 0 \\
\hline chloramphenicol & 1 & 1 \\
\hline self & 0 & 0 \\
\hline 87 & 0 & 0 \\
\hline holds & 0 & 0 \\
\hline earth & 0 & 0 \\
\hline objective & 1 & 0 \\
\hline 12 & 0 & 0 \\
\hline serum & 1 & 1 \\
\hline peptidoglycan & 1 & 1 \\
\hline anthrax & 1 & \\
\hline components & 0 & 0 \\
\hline observing & 1 & 0 \\
\hline simplex & 1 & 1 \\
\hline higher & 0 & 0 \\
\hline reticulum & 1 & 1 \\
\hline james & 0 & 0 \\
\hline helix & 1 & 1 \\
\hline cellular & 0 & 1 \\
\hline all & 0 & 0 \\
\hline transmitted & 0 & 0 \\
\hline violet & 1 & 1 \\
\hline contrast & 1 & 0 \\
\hline aureus & 1 & 1 \\
\hline strand & 1 & 0 \\
\hline matter & $\begin{array}{l}1 \\
0\end{array}$ & 0 \\
\hline branch & 1 & 0 \\
\hline organized & 0 & 0 \\
\hline early & 0 & 0 \\
\hline dinoflagellates & 0 & 1 \\
\hline blue & 0 & 0 \\
\hline roundworm & 1 & \\
\hline watery & 0 & 0 \\
\hline hookworm & 1 & \\
\hline catabolism & 1 & \\
\hline wound & 1 & 0 \\
\hline spectrum & 1 & \\
\hline vector & 1 & \\
\hline region & 1 & ( \\
\hline attach & 0 & \\
\hline substance & 1 & \\
\hline inherit & 0 & \\
\hline result & 0 & \\
\hline
\end{tabular}




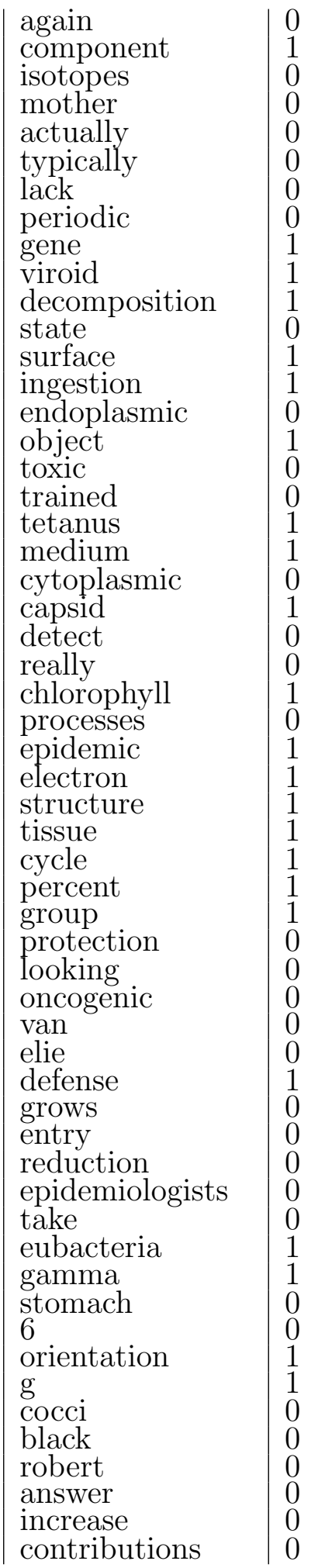

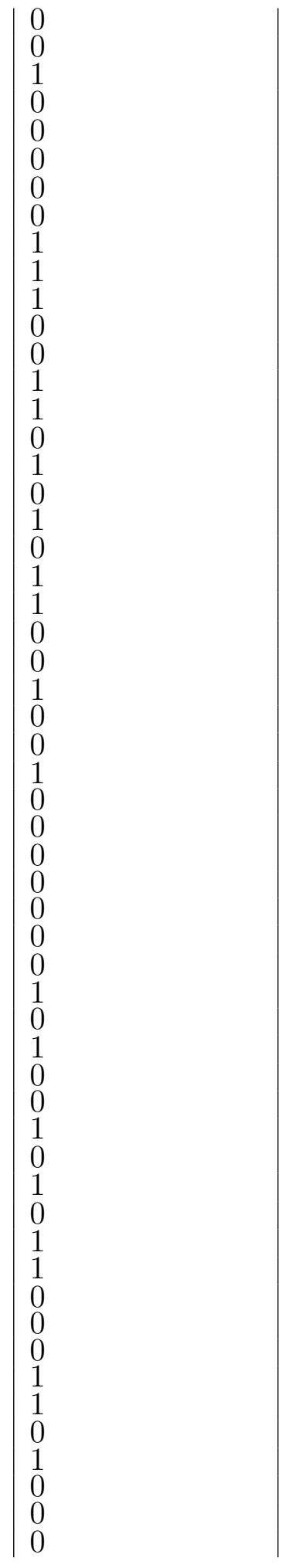




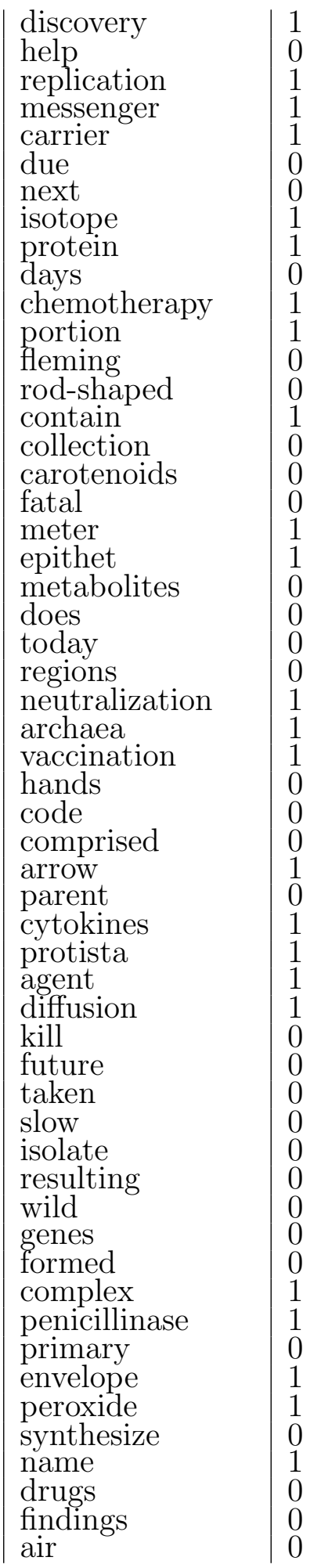

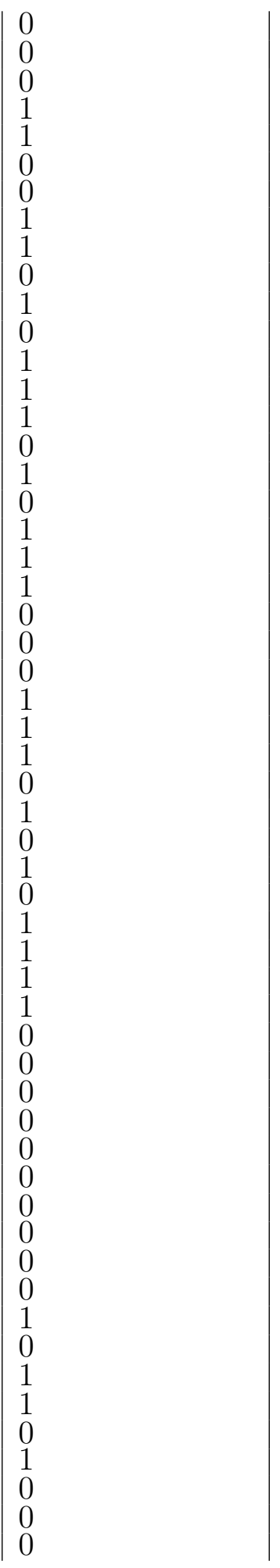




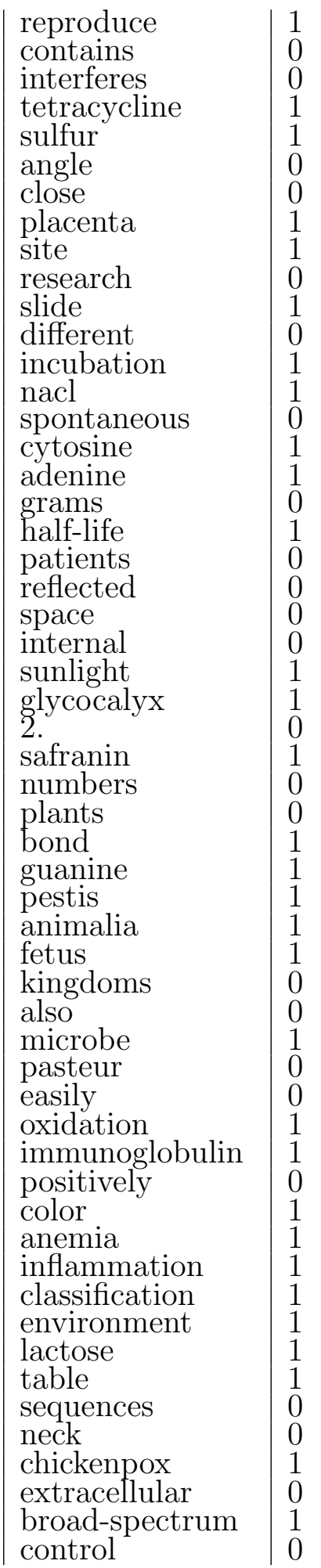

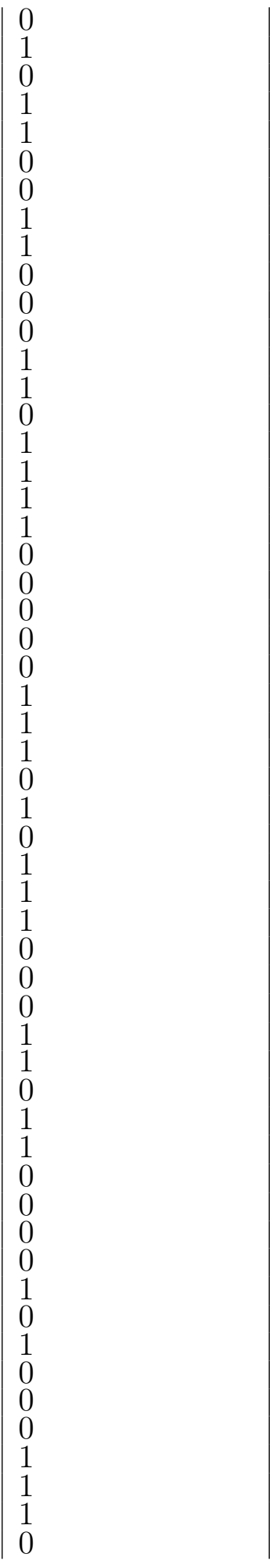




\begin{tabular}{|c|c|c|}
\hline method & 1 & 0 \\
\hline focus & 0 & 0 \\
\hline measured & 0 & 0 \\
\hline second & 0 & 0 \\
\hline make & 0 & 0 \\
\hline takes & 0 & 0 \\
\hline test & 1 & 0 \\
\hline lipid & 1 & 1 \\
\hline germ & 1 & 1 \\
\hline involved & 0 & 0 \\
\hline rubella & 1 & 1 \\
\hline oral . & 0 & 0 \\
\hline injection & 1 & 1 \\
\hline order & 0 & 0 \\
\hline combination & 0 & 0 \\
\hline friend & 0 & 0 \\
\hline microscope & 1 & 1 \\
\hline population & 1 & 0 \\
\hline layer & 1 & 0 \\
\hline organelles & 0 & 1 \\
\hline invades & 0 & 0 \\
\hline agar & 1 & 1 \\
\hline atoms & 0 & 1 \\
\hline penetrate & 0 & 0 \\
\hline soil & 1 & 1 \\
\hline dish & 1 & 0 \\
\hline halophiles & 1 & 1 \\
\hline superoxide & 1 & 1 \\
\hline cluster & 1 & 0 \\
\hline eukaryotic & 0 & 1 \\
\hline reactants & 0 & 1 \\
\hline entire & 0 & 0 \\
\hline were & 0 & 0 \\
\hline poisoning & 1 & 1 \\
\hline phase-contrast & 0 & 1 \\
\hline army & 0 & 0 \\
\hline paralysis & 1 & 0 \\
\hline action & 0 & 0 \\
\hline identified & 0 & 0 \\
\hline under & 0 & 0 \\
\hline pseudopods & 1 & 1 \\
\hline use. & 0 & 1 \\
\hline mask & 1 & 0 \\
\hline ) & 0 & 1 \\
\hline passive & 1 & 0 \\
\hline stain & 1 & 1 \\
\hline $\begin{array}{l}\text { fission } \\
\text { formation }\end{array}$ & $\begin{array}{l}1 \\
1\end{array}$ & $\begin{array}{l}1 \\
0\end{array}$ \\
\hline notatum & 1 & 1 \\
\hline host & 1 & 0 \\
\hline mild & 0 & 0 \\
\hline $\begin{array}{l}\text { infectious } \\
\text { functional }\end{array}$ & $\begin{array}{l}0 \\
0\end{array}$ & $\begin{array}{l}1 \\
0\end{array}$ \\
\hline
\end{tabular}


Table A.1: The table shows the full results of the distributed specificity algorithm compared with the TermoStat's in the microbiology domain. $n_{\text {train }}=k$ signifies the training set size of $k$.

\section{A.2 Optometry}

\begin{tabular}{|l|l|l|}
\hline Term Candi- & Termostat & Distributed \\
date & & Specificity \\
\hline this & 0 & 0 \\
metamorphopsia & 1 & 1 \\
pp & 0 & 0 \\
pursuit & 1 & 0 \\
canthus & 1 & 1 \\
down & 0 & 1 \\
result & 1 & 0 \\
retina & 1 & 1 \\
2004 & 0 & 0 \\
disadvantage & 1 & 0 \\
database & 1 & 0 \\
blink & 1 & 1 \\
combination & 1 & 0 \\
figure & 1 & 0 \\
coefficient & 1 & 0 \\
novice & 0 & 1 \\
cs & 0 & 1 \\
thereby & 0 & 0 \\
bar & 1 & 0 \\
j.d & 0 & 0 \\
review & 1 & 0 \\
discuss & 1 & 0 \\
factor & 1 & 0 \\
alignment & 1 & 1 \\
5.10 & 0 & 0 \\
driving & 0 & 1 \\
analysis & 1 & 0 \\
inspect & 1 & 1 \\
progression & 1 & 0 \\
correcting & 0 & 1 \\
rheostat & 1 & 1 \\
sheet & 1 & 0 \\
/ & 0 & 0 \\
determines & 0 & 0 \\
\hline
\end{tabular}




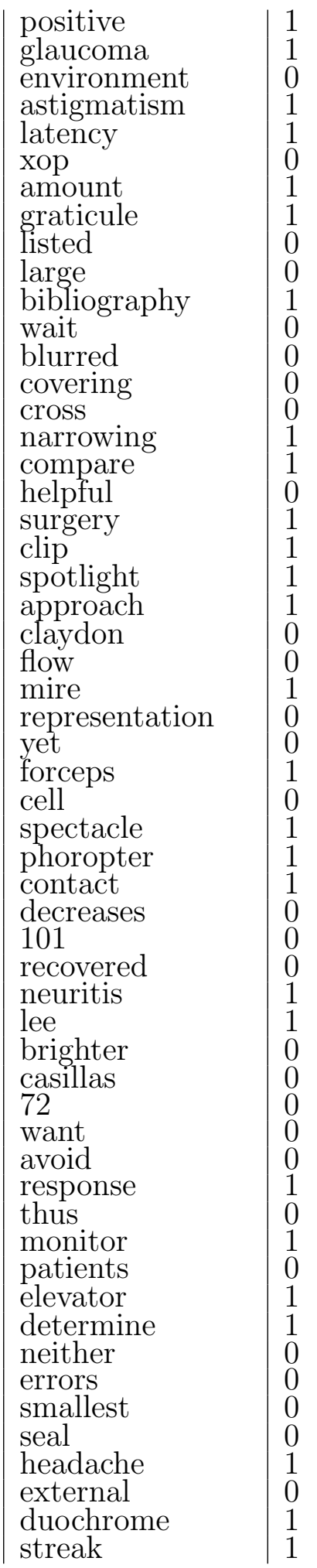

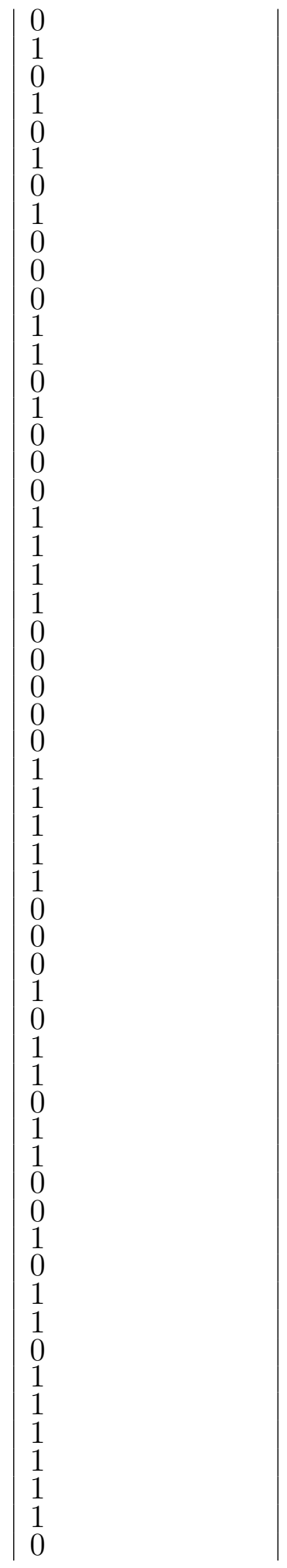




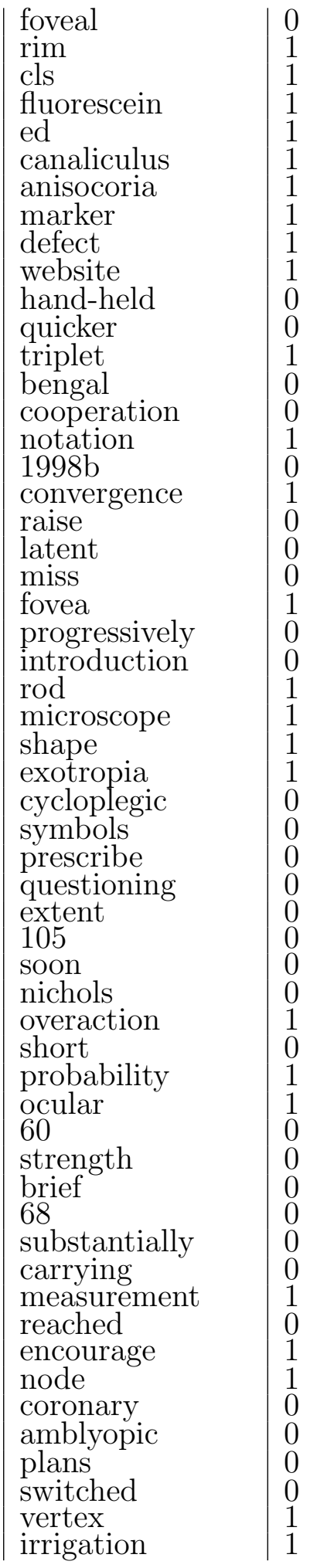

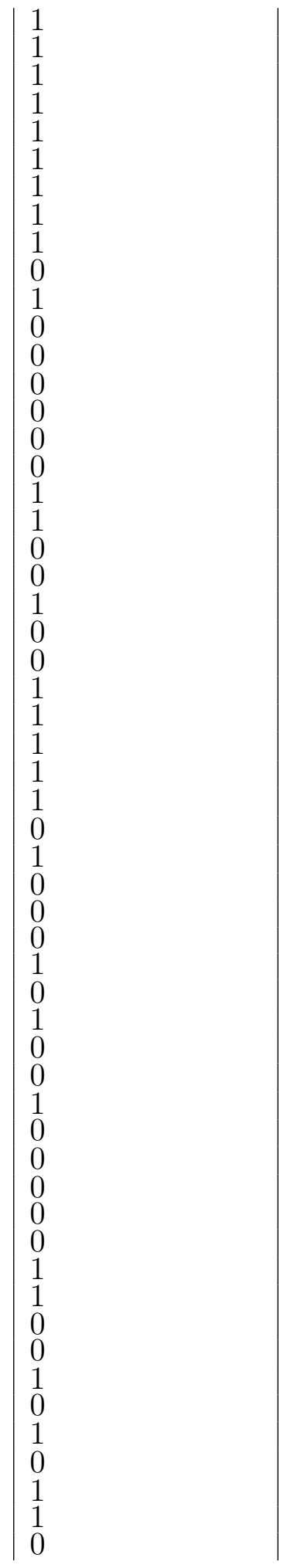




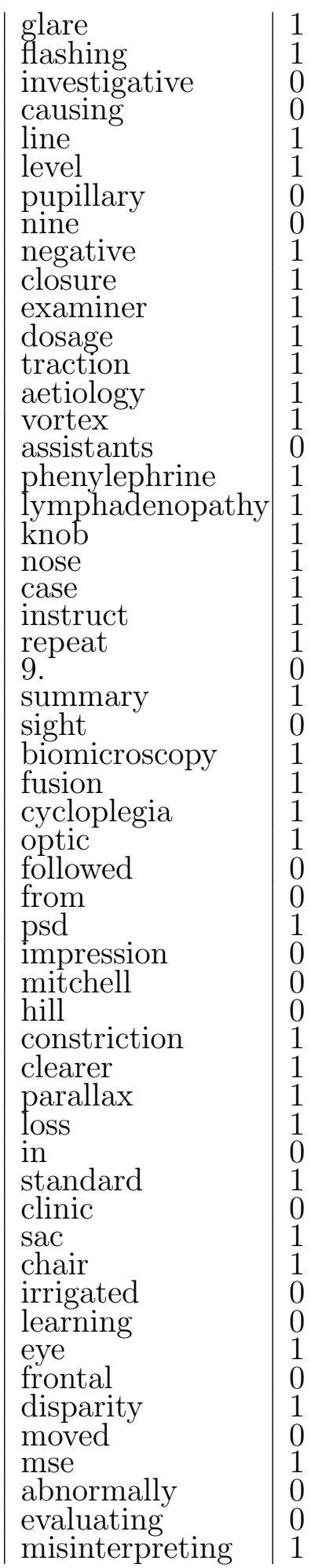

1
1
1
1
1
1
1
0
0
1
1
0
0
0
0
1
0
0
1
1
1
1
0
1
0
1
1
0
1
1
0
1
1
0
0
0
1
1
1
0
1
1
1
1
1
0
0
1
1
0
0
0
0
0
0




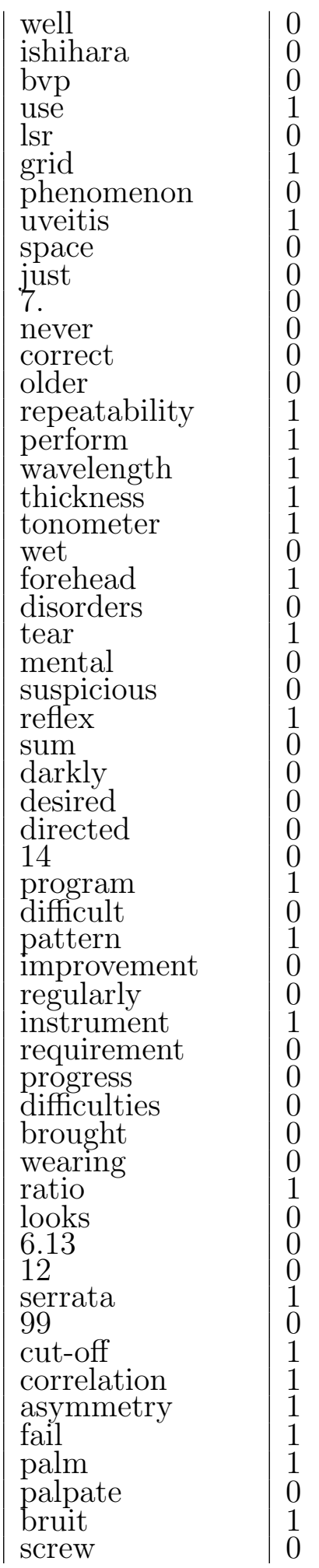

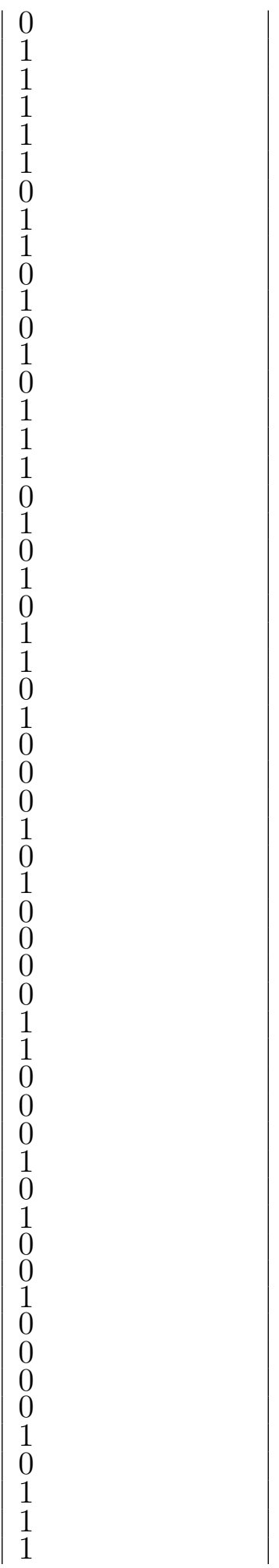




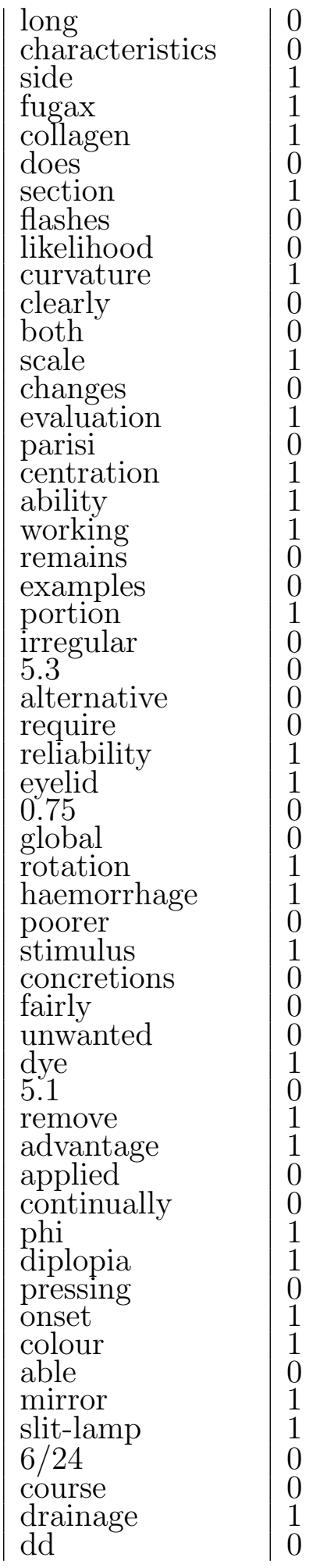

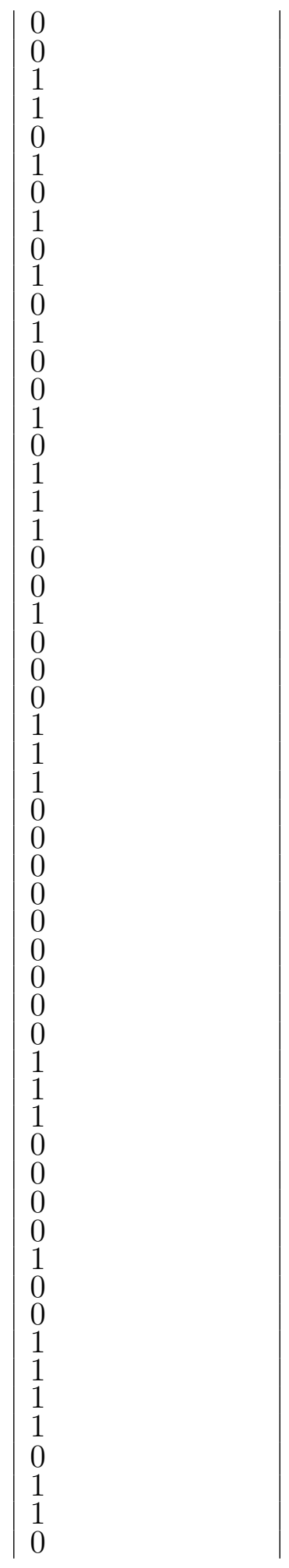




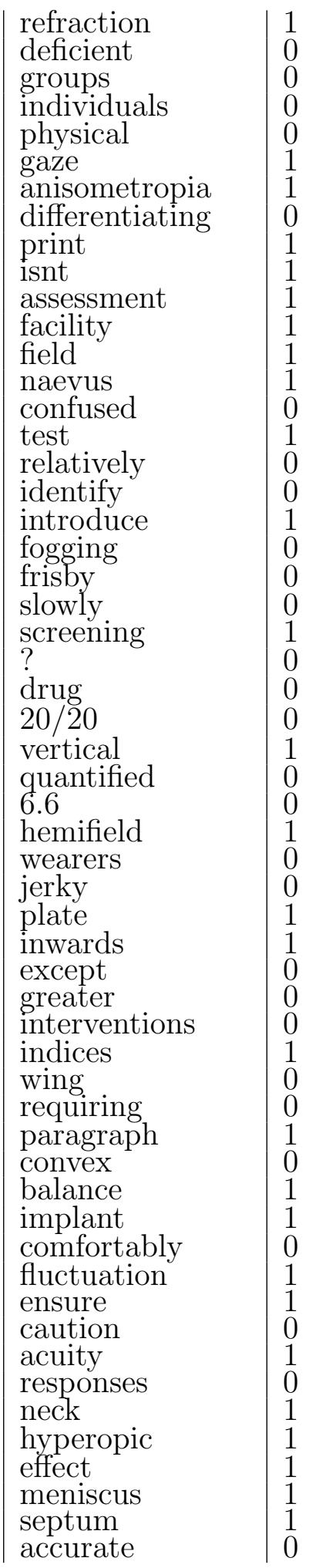

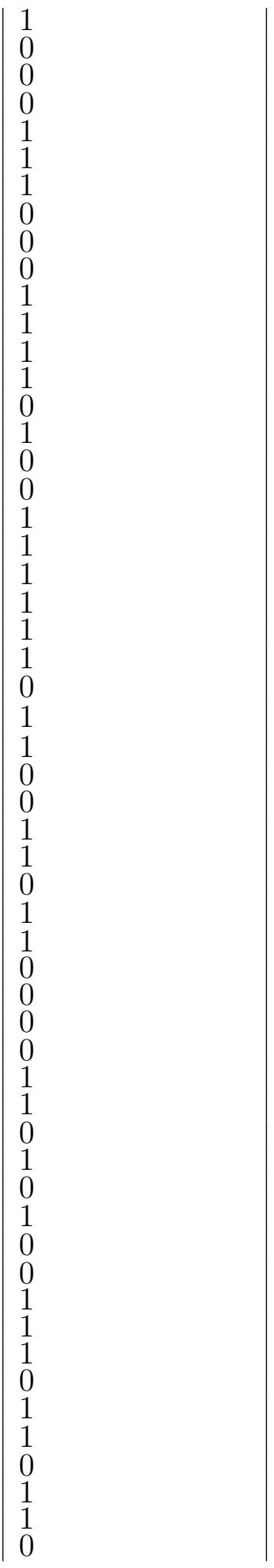




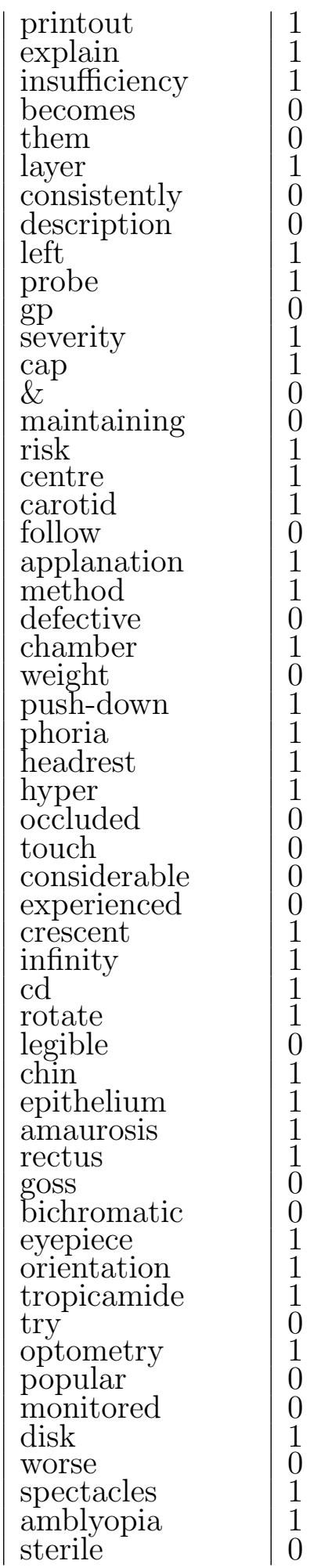

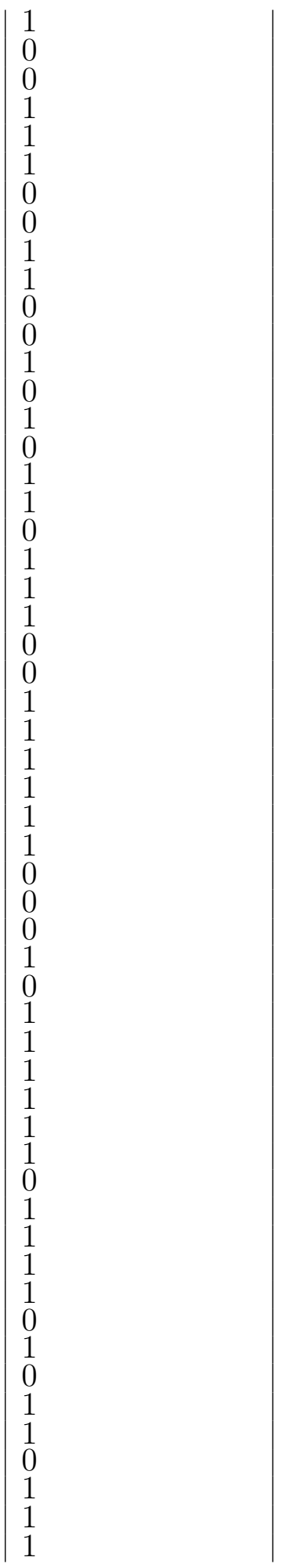




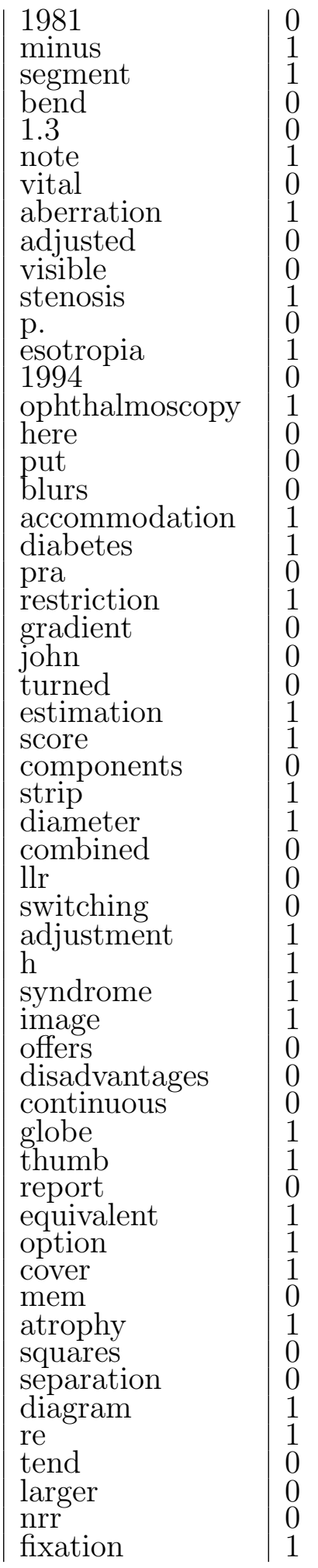

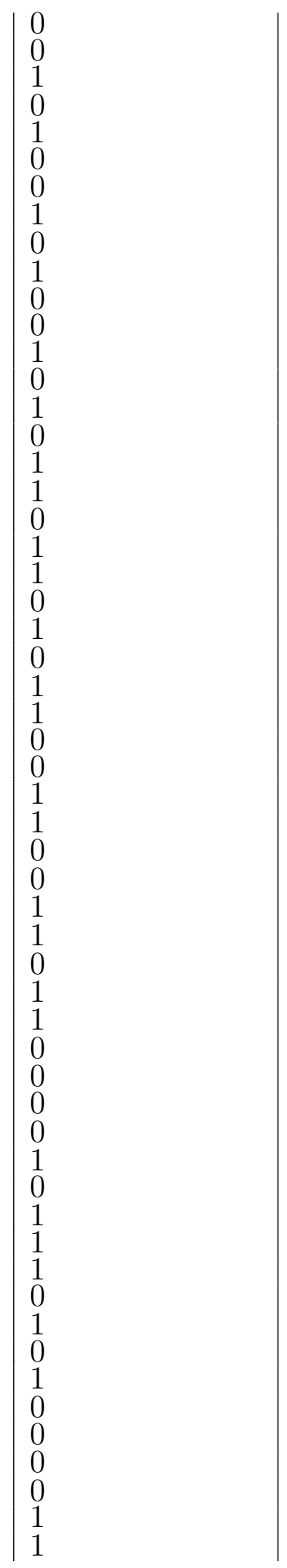




\begin{tabular}{|c|c|c|}
\hline distance & 1 & 1 \\
\hline palsy & 1 & 1 \\
\hline add & 1 & 0 \\
\hline epiphora & 1 & 1 \\
\hline isolate & 0 & 0 \\
\hline refractions & 0 & 1 \\
\hline balancing & 1 & 0 \\
\hline brightness & 1 & 1 \\
\hline lymph & 1 & 0 \\
\hline keratoconus & 1 & 1 \\
\hline punctum & 1 & 1 \\
\hline membrane & 1 & 1 \\
\hline ametropia & 1 & 1 \\
\hline puncta & 1 & 1 \\
\hline 17. & 0 & 0 \\
\hline ruler & 1 & 1 \\
\hline dots & 0 & 1 \\
\hline children & 0 & 0 \\
\hline lamp & 1 & 1 \\
\hline clarity & 1 & 1 \\
\hline location & 1 & 0 \\
\hline what & 0 & 0 \\
\hline especially & 0 & 0 \\
\hline pulsation & 1 & 0 \\
\hline sports & 0 & 0 \\
\hline angle & 1 & 1 \\
\hline legal & 0 & 0 \\
\hline words & 0 & 0 \\
\hline adding & 0 & 0 \\
\hline remainder & 0 & 0 \\
\hline & 0 & 0 \\
\hline+ & 0 & 1 \\
\hline outer . & 0 & 1 \\
\hline minimise & 0 & 1 \\
\hline relationship & 0 & 0 \\
\hline disappearance & 1 & 0 \\
\hline calibration & 1 & 1 \\
\hline pathway & 1 & 0 \\
\hline assuming & 0 & 0 \\
\hline format & 1 & 1 \\
\hline plates & 0 & 1 \\
\hline literature & 1 & 0 \\
\hline dilate & 1 & 1 \\
\hline 35 & 0 & 0 \\
\hline reading & 1 & 1 \\
\hline spur . & 1 & 0 \\
\hline attempting & 1 & 1 \\
\hline bailey & $\begin{array}{l}1 \\
0\end{array}$ & $\begin{array}{l}0 \\
0\end{array}$ \\
\hline threshold & 1 & 1 \\
\hline subjects & 0 & 0 \\
\hline precise & 0 & 0 \\
\hline prisms & 0 & 1 \\
\hline vary & 0 & 0 \\
\hline correlate & 1 & 0 \\
\hline
\end{tabular}




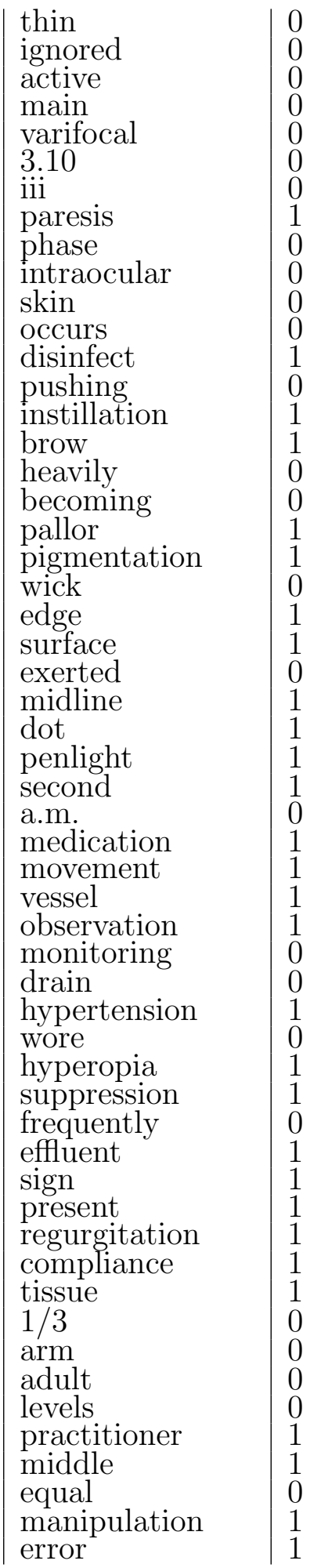

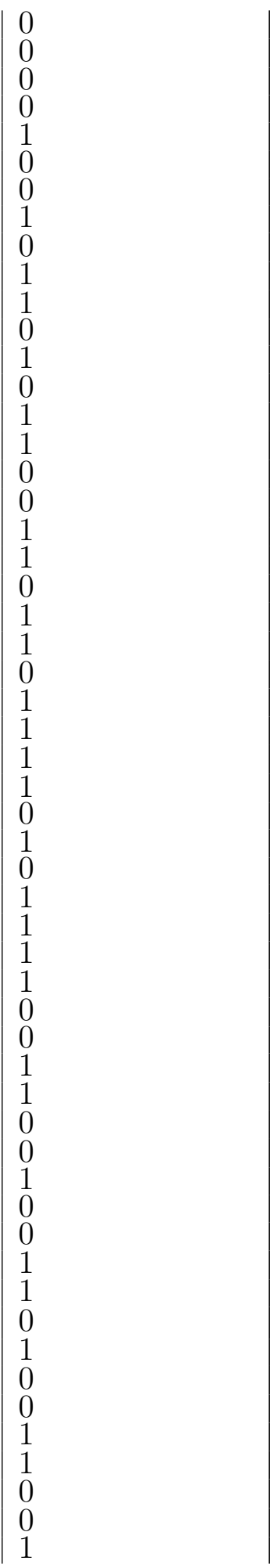




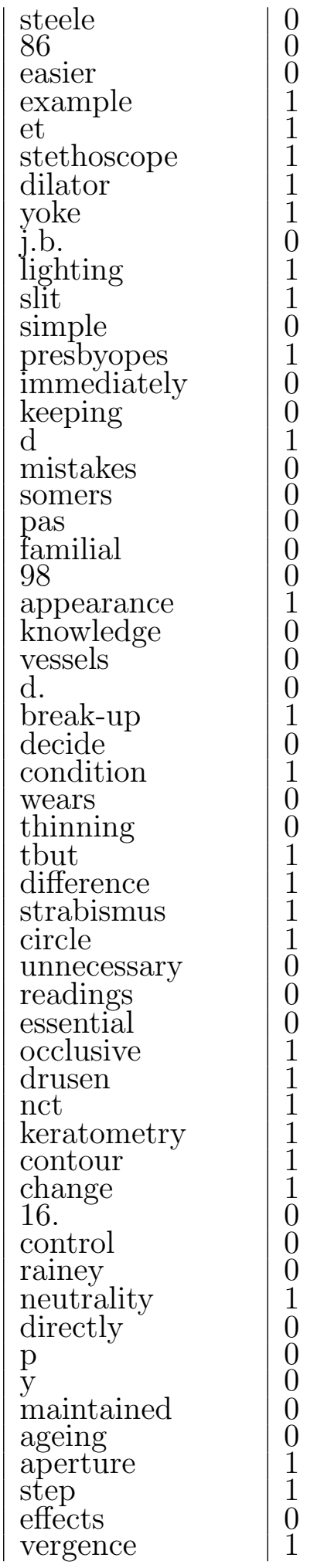

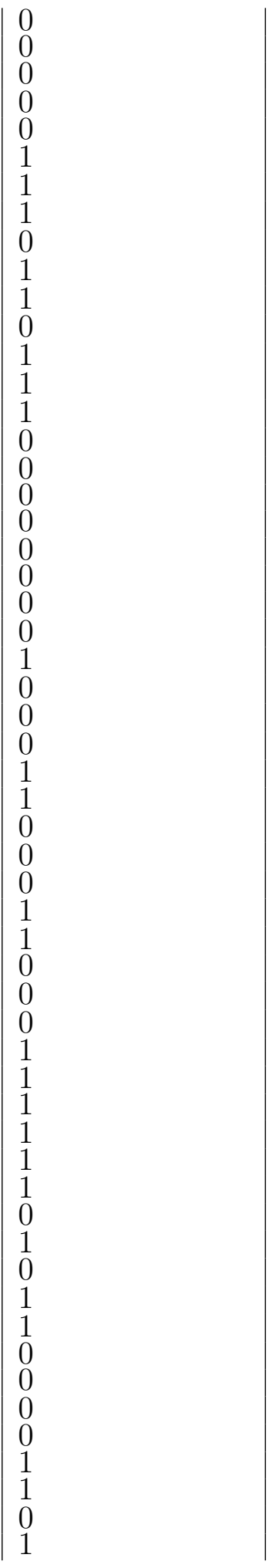




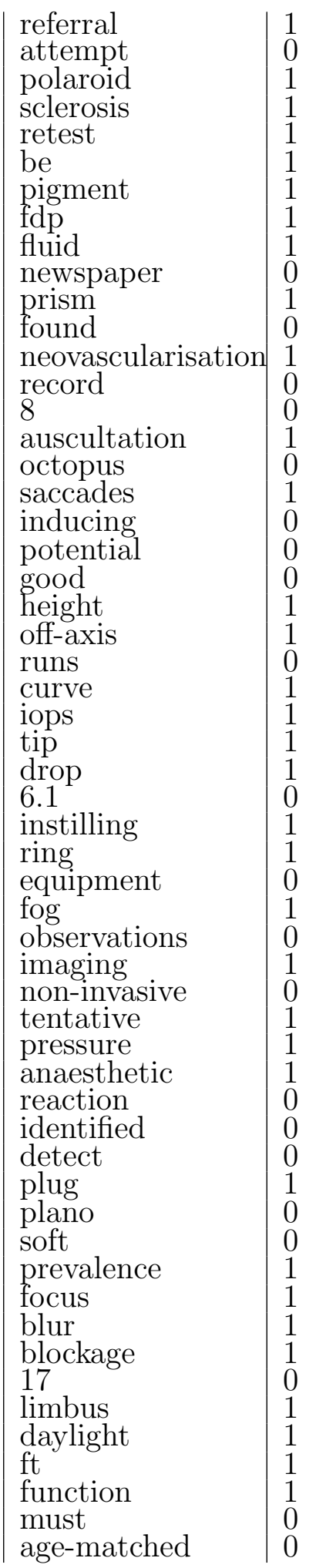

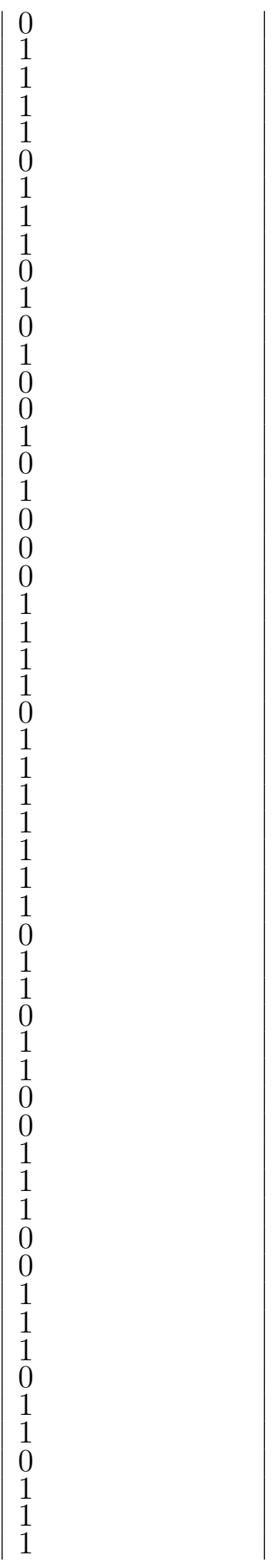




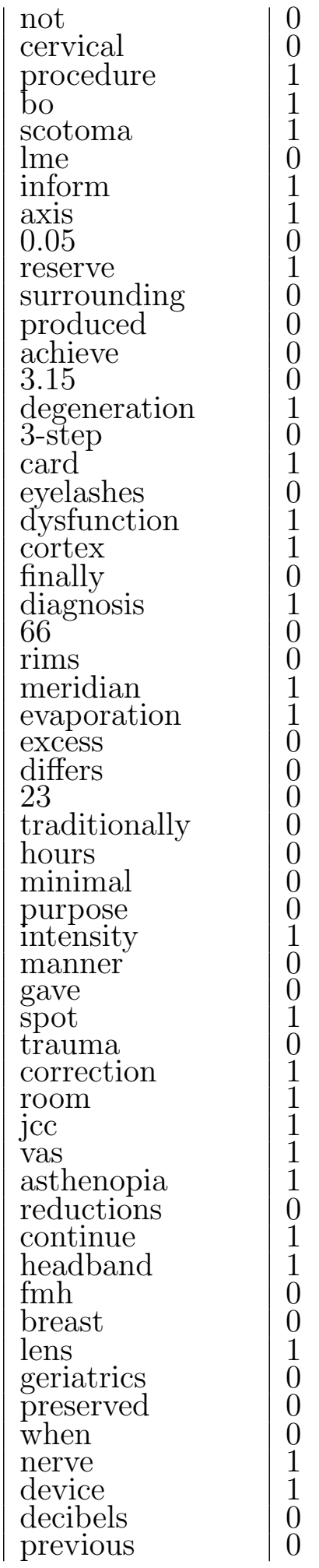

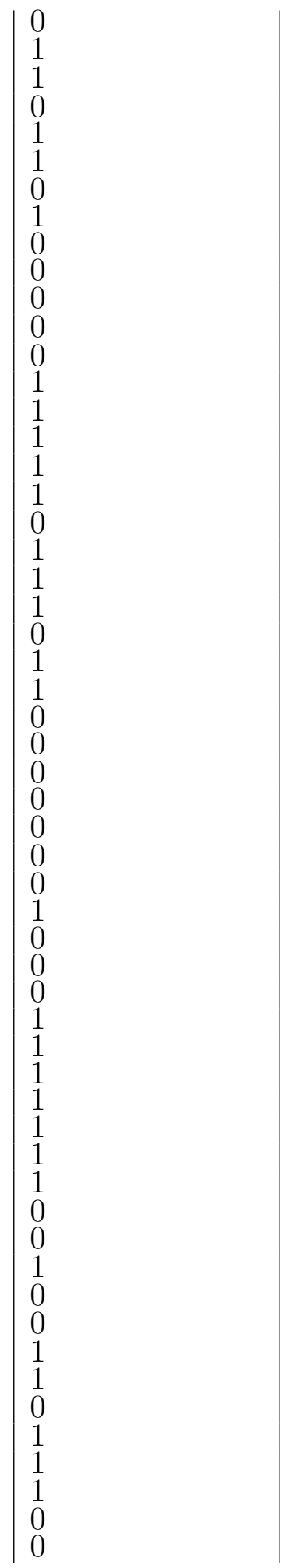




\begin{tabular}{|l|l} 
system & 1 \\
testing & 1 \\
watch & 0 \\
desk & 0 \\
professional & 0 \\
6.6.4 & 0 \\
millimetre & 1 \\
photography & 0 \\
apex & 1 \\
wilkes & 0 \\
jump & 1 \\
recovery & 1 \\
evaluate & 1 \\
cc & 1 \\
changed & 0 \\
summation & 1 \\
differentiate & 0 \\
vertically & 0 \\
chart & 1 \\
edges & 0 \\
interpretation & 1 \\
regular & 0 \\
principle & 0 \\
periphery & 1 \\
perception & 0 \\
defectives & 1 \\
size & 1 \\
mmhg & 1 \\
eds & 0 \\
db & 1 \\
snellen & 0 \\
forgetting & 1 \\
intended & 0 \\
choroid & 1 \\
phenol & 1 \\
abnormality & 1 \\
mercury & 0 \\
dissociation & 1 \\
excessive & 0 \\
updated & 0 \\
misalignment & 1 \\
cyclopentolate & 1 \\
laterality & 1 \\
power & 1 \\
validity & 1 \\
cataracts & 0 \\
scotomas & 0 \\
tonometry & 1 \\
age & 1 \\
saunders & 0 \\
os & 1 \\
simpler & 0 \\
corresponding & 0 \\
tenderness & 1 \\
yrs & 1
\end{tabular}

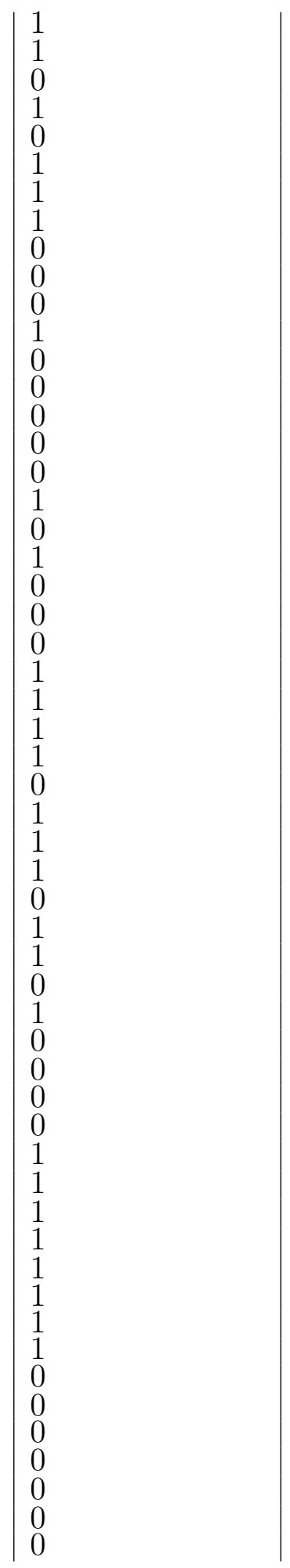




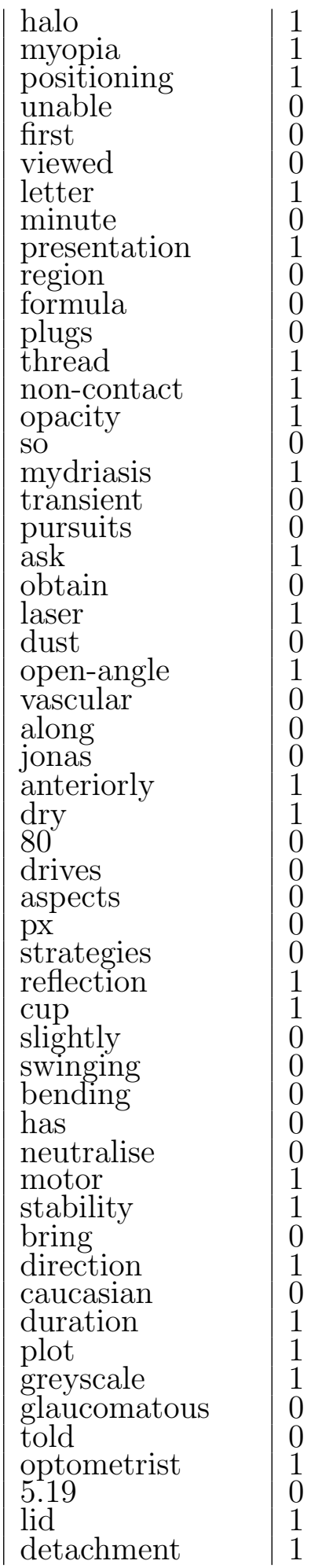

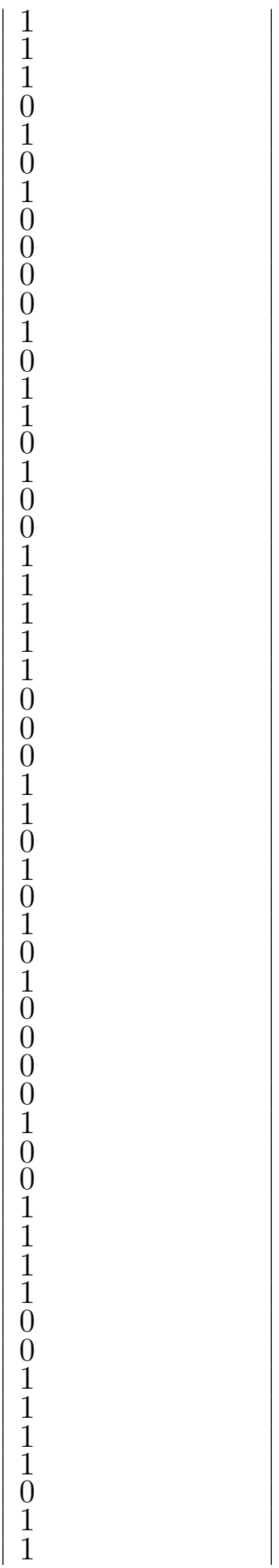




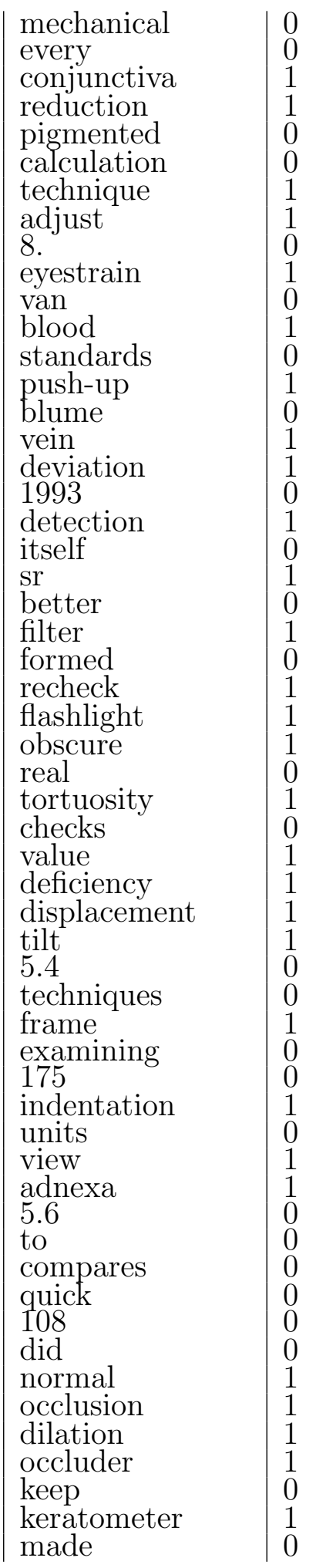

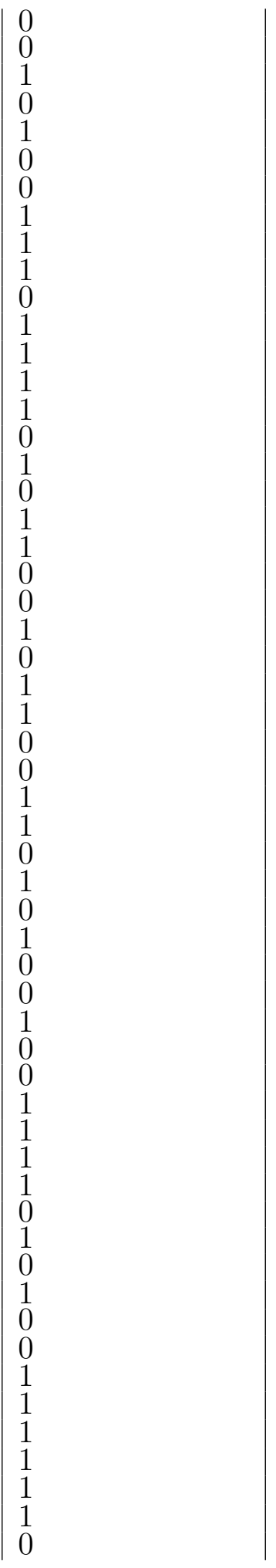




\begin{tabular}{|c|c|c|}
\hline selection & 0 & 0 \\
\hline adaptations & 0 & 0 \\
\hline cobalt & 1 & 0 \\
\hline 106 & 0 & 0 \\
\hline repeatable & 0 & 0 \\
\hline synechiae & 1 & 1 \\
\hline sensitive & 0 & 1 \\
\hline retesting & 1 & 1 \\
\hline arrow & 1 & 1 \\
\hline employed & 0 & 0 \\
\hline expected & 0 & 0 \\
\hline cataract & 1 & 1 \\
\hline beta & 0 & 1 \\
\hline bowl & 0 & 0 \\
\hline pupil & 1 & 1 \\
\hline press & 0 & 0 \\
\hline inappropriate & 1 & 0 \\
\hline birch & 0 & 0 \\
\hline discomfort & 1 & 1 \\
\hline established & 0 & 0 \\
\hline flanagan & 0 & 0 \\
\hline rso & 0 & 0 \\
\hline distortion & 1 & 0 \\
\hline powered & 0 & 0 \\
\hline commonly & 0 & 0 \\
\hline types & 0 & 0 \\
\hline opacities & 0 & 1 \\
\hline extreme & 0 & 0 \\
\hline suprathreshold & 1 & 1 \\
\hline ensures & 0 & 1 \\
\hline wide & 0 & 0 \\
\hline 90 & 0 & 0 \\
\hline examined & 0 & 0 \\
\hline increases & 0 & 0 \\
\hline magnification & 1 & 1 \\
\hline oblique & 1 & 1 \\
\hline $\mathrm{cm}$ & 1 & 1 \\
\hline 26 & 0 & 0 \\
\hline unexplained & 0 & 0 \\
\hline superiorly & 0 & 1 \\
\hline target & 1 & 1 \\
\hline pds & 1 & 0 \\
\hline cardiac & 0 & 0 \\
\hline $6 / 9$ & 0 & 0 \\
\hline think & 0 & 0 \\
\hline mobility & 0 & 1 \\
\hline doubling & 1 & 0 \\
\hline indentor & 1 & 1 \\
\hline myope & 1 & 1 \\
\hline least & 0 & 0 \\
\hline diagnoses & 0 & 0 \\
\hline eso & 1 & 0 \\
\hline axial & 0 & 0 \\
\hline worth & 0 & 0 \\
\hline pfr & 1 & \\
\hline
\end{tabular}




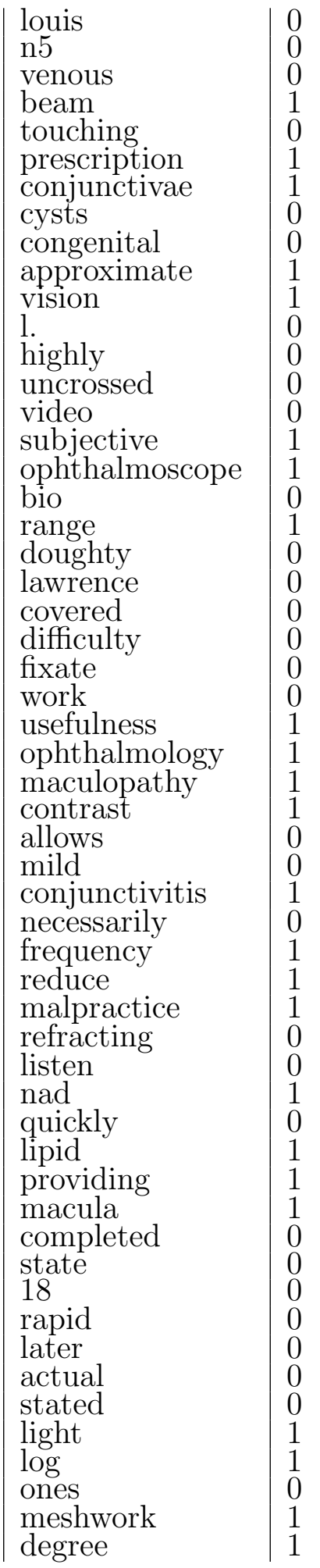

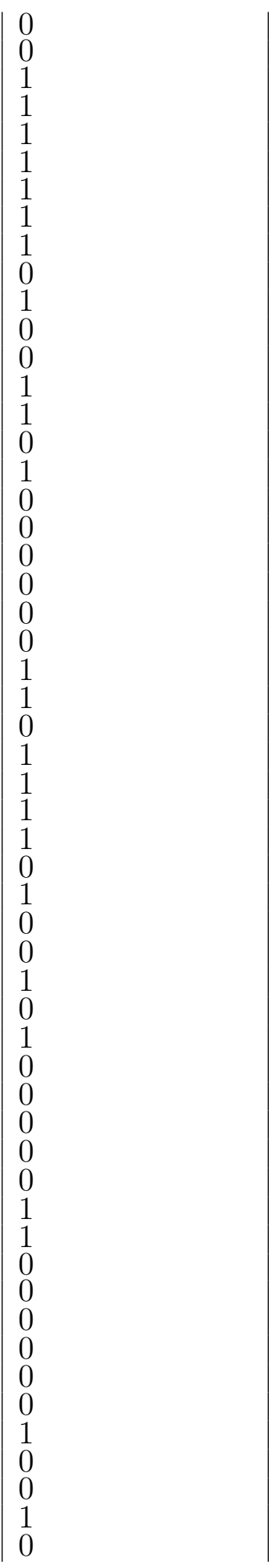




\begin{tabular}{|c|c|}
\hline subcapsular & 1 \\
\hline kappa & 1 \\
\hline sphere. & 1 \\
\hline ectropion & 1 \\
\hline flat & 0 \\
\hline finger & 1 \\
\hline saline & 1 \\
\hline muscle & 1 \\
\hline artefact & 1 \\
\hline & 1 \\
\hline all & 0 \\
\hline recommended & 0 \\
\hline anaesthesia & 1 \\
\hline collection & 0 \\
\hline heard & 0 \\
\hline caps & 0 \\
\hline $\mathrm{oh}$ & 1 \\
\hline nodes & $\begin{array}{l}0 \\
0\end{array}$ \\
\hline $\begin{array}{l}\text { outwards } \\
\text { blue }\end{array}$ & $\begin{array}{l}0 \\
0\end{array}$ \\
\hline head & 1 \\
\hline absolute & 0 \\
\hline inaccuracies & 0 \\
\hline information & 1 \\
\hline density & 0 \\
\hline anatomy & 1 \\
\hline handle & 1 \\
\hline 1990 & 0 \\
\hline yourself & 0 \\
\hline seated & 0 \\
\hline posterior & 1 \\
\hline follow-up & 1 \\
\hline $\begin{array}{l}\text { prescribing } \\
\text { disease }\end{array}$ & $\begin{array}{l}0 \\
1\end{array}$ \\
\hline satisfaction & 0 \\
\hline $1 / 2$ & 0 \\
\hline birth & 0 \\
\hline coloured & 0 \\
\hline cupping & 1 \\
\hline $1 / \mathrm{r}$ & 0 \\
\hline disability & 1 \\
\hline fundus & 1 \\
\hline cannula & 1 \\
\hline measure & 1 \\
\hline perimeter & 1 \\
\hline neuropathy & 1 \\
\hline classification & 1 \\
\hline themselves & 0 \\
\hline university & 0 \\
\hline block & 1 \\
\hline $6 / 3$ & 0 \\
\hline pictures & 0 \\
\hline arteries & 0 \\
\hline quantity & 0 \\
\hline arbiter & 1 \\
\hline
\end{tabular}




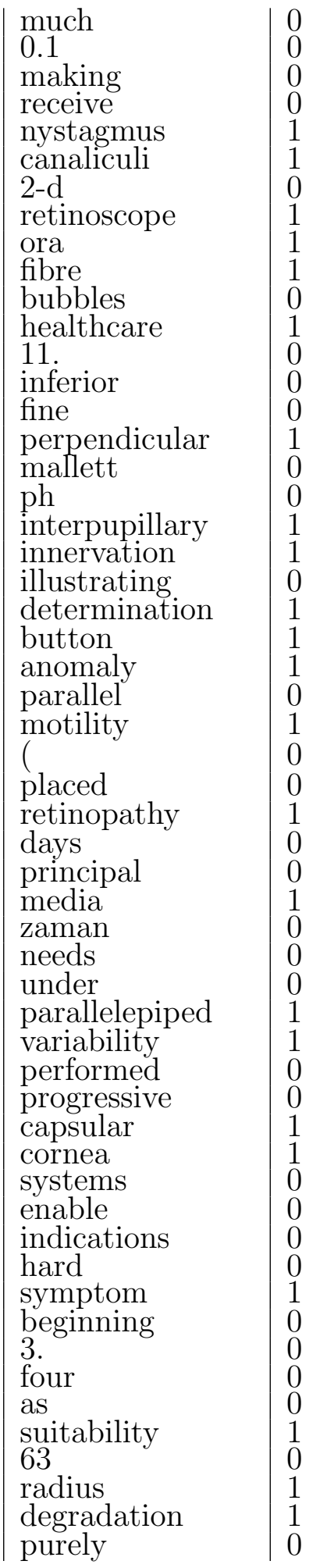

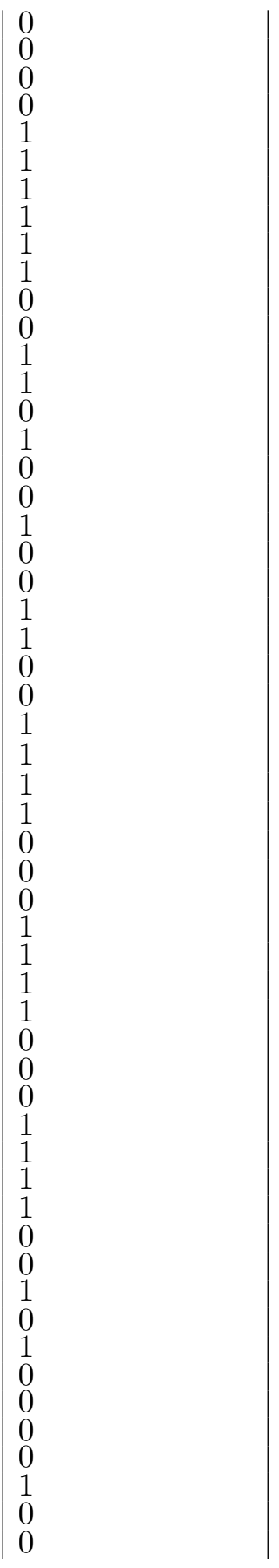




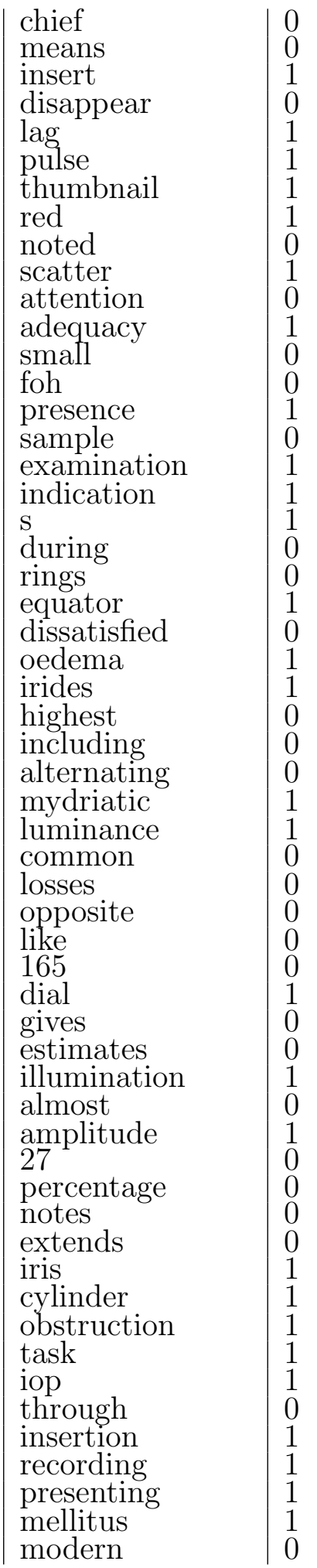

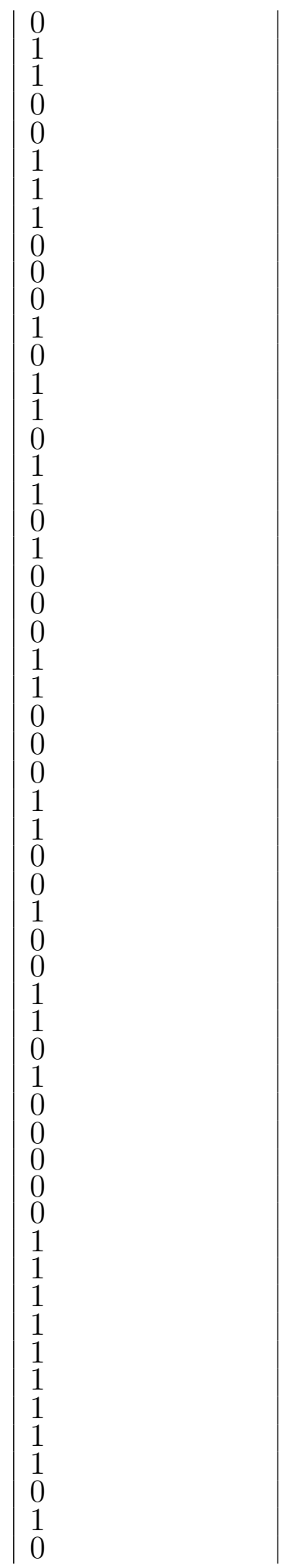




\begin{tabular}{|c|c|c|}
\hline divergence & 1 & 0 \\
\hline confusion & 1 & 1 \\
\hline coupling & 1 & 0 \\
\hline bottom & 0 & 0 \\
\hline position & 1 & 1 \\
\hline practice & 1 & 1 \\
\hline & 1 & 0 \\
\hline indicate & 1 & 0 \\
\hline pinguecula & 1 & 1 \\
\hline patient & 0 & 1 \\
\hline pinhole & 1 & 1 \\
\hline visibility & 1 & 1 \\
\hline matrix & 0 & 0 \\
\hline taking & 0 & 1 \\
\hline specificity & 1 & 0 \\
\hline perimetry & 1 & 1 \\
\hline states & 0 & 0 \\
\hline quadrant & 1 & 1 \\
\hline $\begin{array}{l}2.5 \\
\text { cheek }\end{array}$ & $\begin{array}{l}0 \\
0\end{array}$ & $\begin{array}{l}1 \\
1\end{array}$ \\
\hline hypertensive & 1 & 0 \\
\hline & 0 & 0 \\
\hline comparison & 1 & 0 \\
\hline perfect & 0 & 0 \\
\hline decrease & 1 & 0 \\
\hline acute & 0 & 0 \\
\hline width & 1 & 1 \\
\hline facial & 0 & 1 \\
\hline accuracy & 1 & 1 \\
\hline needed & 0 & 1 \\
\hline $20 / 200$ & 0 & 1 \\
\hline learn & 0 & 0 \\
\hline super & 1 & 0 \\
\hline she & 0 & 1 \\
\hline fashion & 0 & 0 \\
\hline history & 1 & 0 \\
\hline endothelium & 1 & 0 \\
\hline psc & 1 & 0 \\
\hline significance & 0 & 0 \\
\hline duct & 1 & 1 \\
\hline venules & 0 & 1 \\
\hline locate & 1 & 0 \\
\hline sclera & 1 & 1 \\
\hline written & 0 & 0 \\
\hline hold & 0 & 1 \\
\hline producing & 0 & 0 \\
\hline endpoint & 1 & 1 \\
\hline stereopsis & 1 & 1 \\
\hline point & 1 & 1 \\
\hline entire. & 0 & 1 \\
\hline situation & 0 & 0 \\
\hline indicator & 1 & 1 \\
\hline plus & 1 & 0 \\
\hline ensuring & 0 & 0 \\
\hline reassurance & 1 & 1 \\
\hline
\end{tabular}




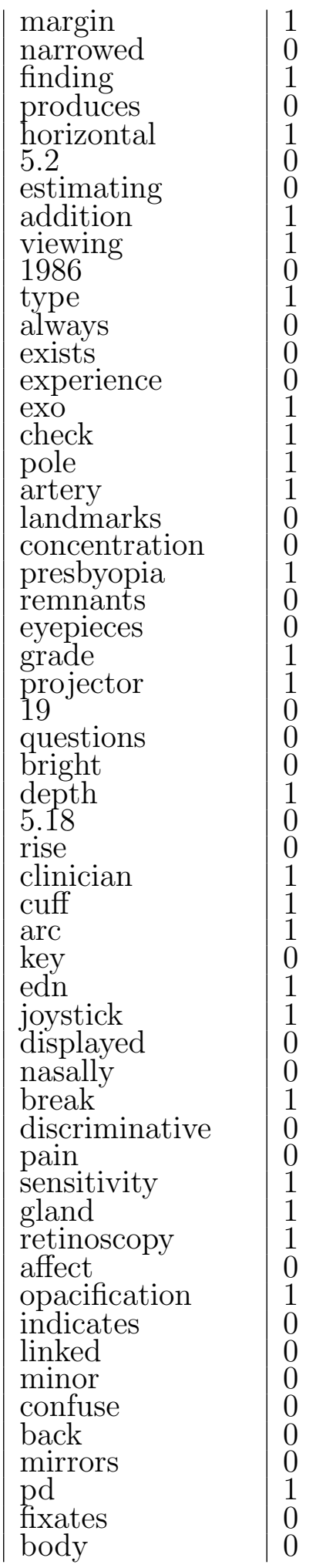

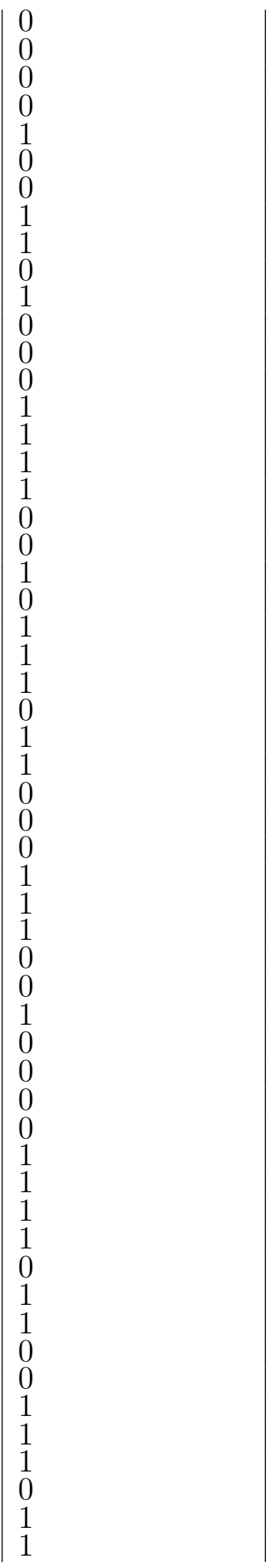




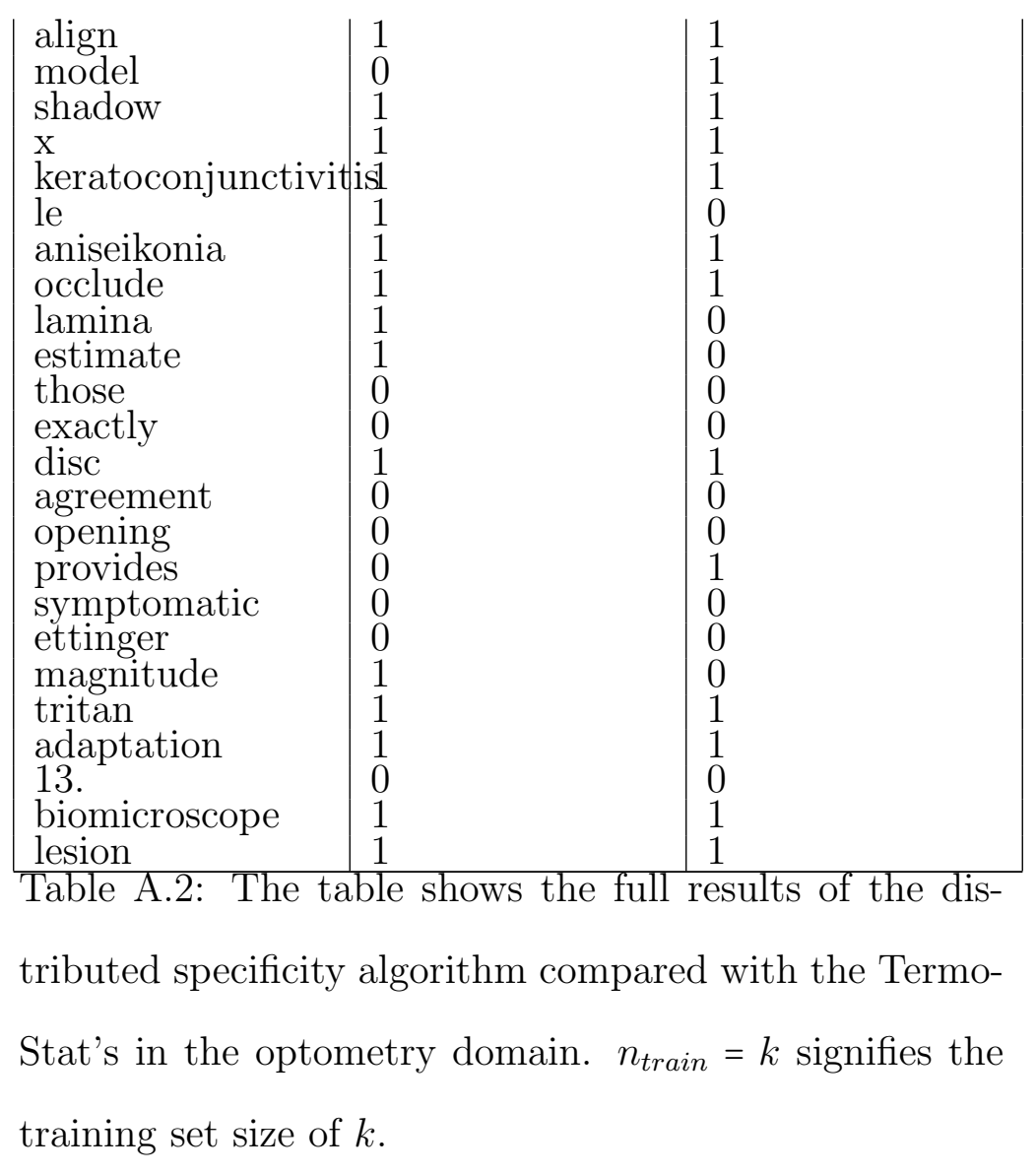

\section{A.3 Economics}

\begin{tabular}{|l|l|l|}
\hline Term Candi- & TermoStat & Distributed \\
date & & Specificity \\
\hline instance & 1 & 0 \\
yen & 1 & 1 \\
vreeland & 0 & 0 \\
possibilities & 0 & 0 \\
notice & 0 & 0 \\
component & 1 & 0 \\
elasticity & 1 & 0 \\
dramatically & 0 & 0 \\
becomes & 0 & 0 \\
1999 & 0 & 1 \\
variable & 1 & 0 \\
credibility & 1 & 0
\end{tabular}




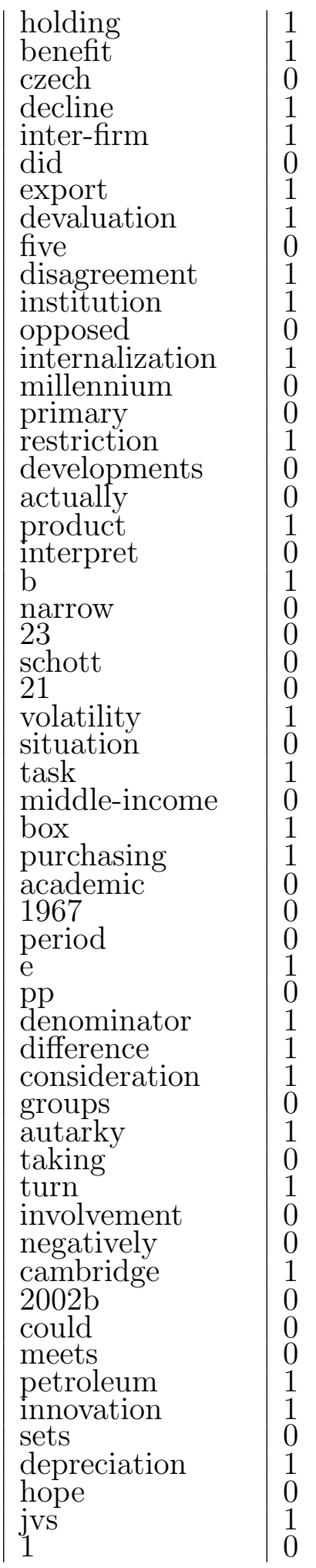

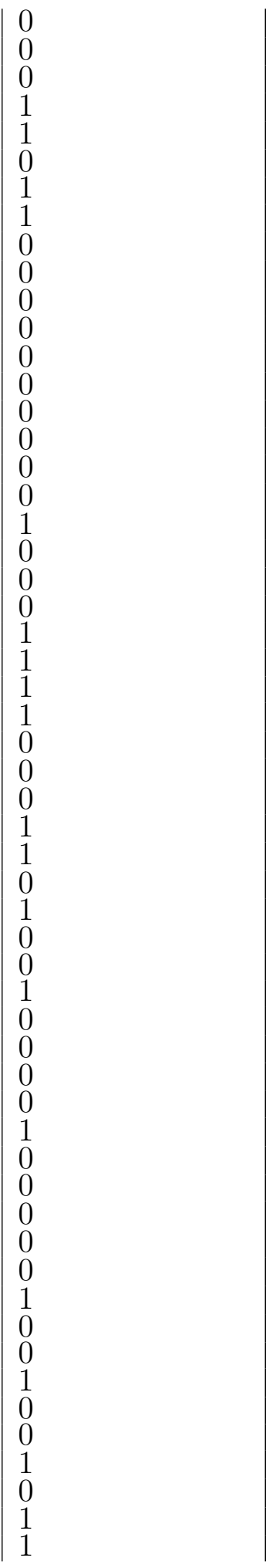




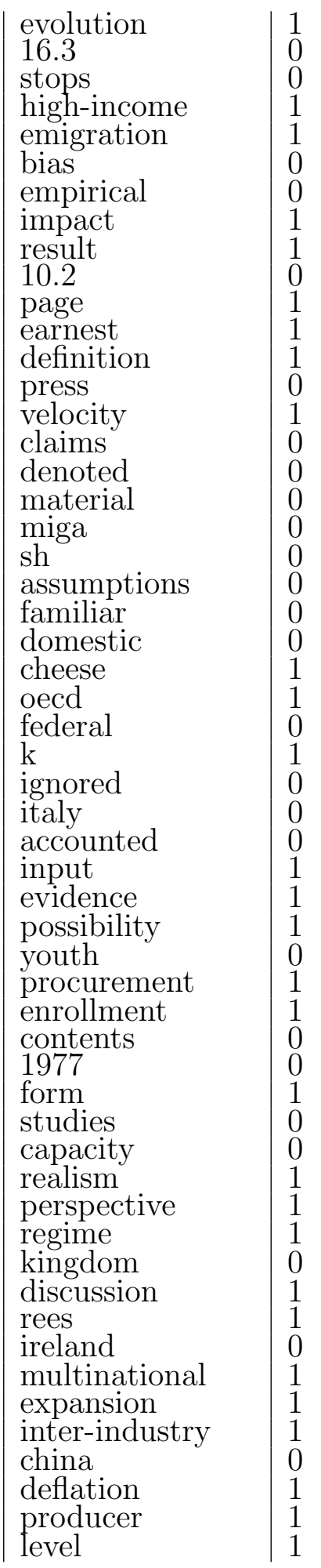

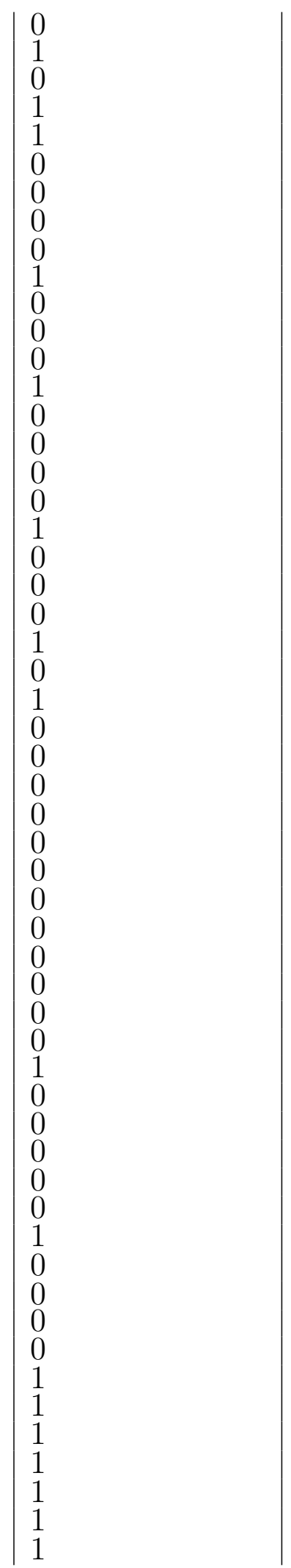




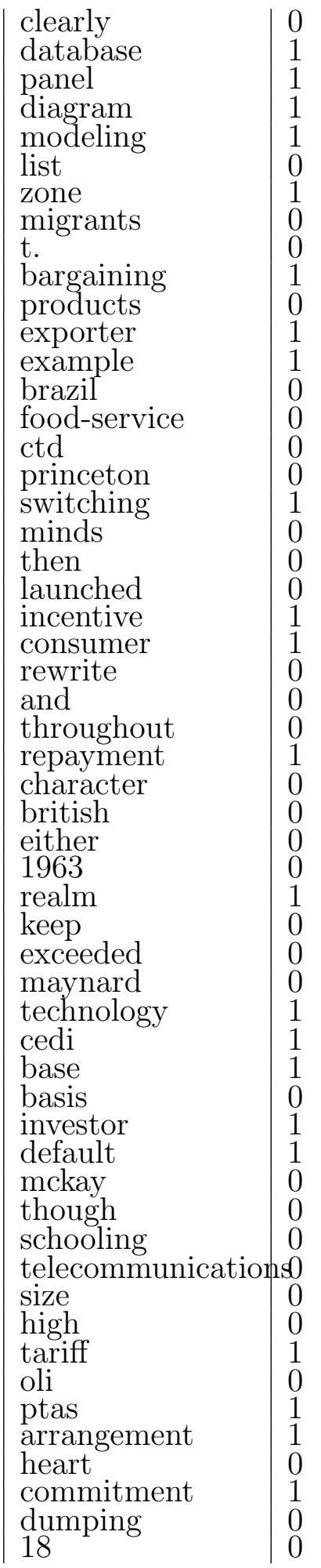

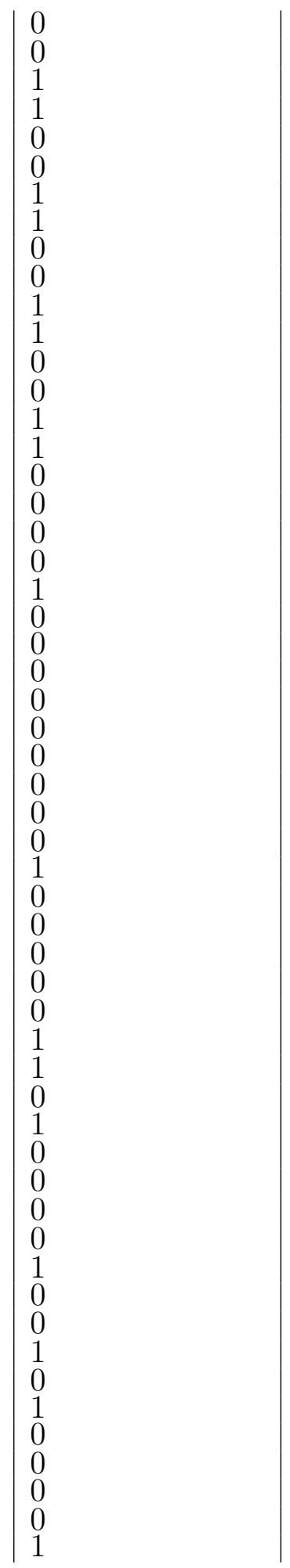




\begin{tabular}{|l|l} 
change & 1 \\
stiglitz & 0 \\
generation & 0 \\
trinity & 1 \\
revealed & 0 \\
proportion & 0 \\
payment & 1 \\
assumption & 1 \\
choice & 0 \\
programs & 0 \\
introductory & 0 \\
phase & 1 \\
bond & 1 \\
automobile & 1 \\
bases & 0 \\
stable & 0 \\
91 & 0 \\
lower & 0 \\
among & 0 \\
world & 1 \\
harry & 0 \\
peg & 1 \\
commerce & 0 \\
mckinnon & 0 \\
quality & 0 \\
mode & 1 \\
know & 0 \\
windows & 0 \\
netherlands & 0 \\
famous & 0 \\
outflow & 1 \\
1993 & 0 \\
movement & 1 \\
risk & 1 \\
uip & 1 \\
multilateralism & 1 \\
expanded & 0 \\
northern & 0 \\
trading & 1 \\
interests & 0 \\
privatization & 1 \\
widely & 0 \\
appendices & 1 \\
regionalism & 1 \\
literacy & 1 \\
switzerland & 0 \\
government & 1 \\
gravity & 1 \\
moved & 0 \\
perfectly & 0 \\
mechanisms & 0 \\
lsm & 1 \\
subset & bank \\
opposite &
\end{tabular}

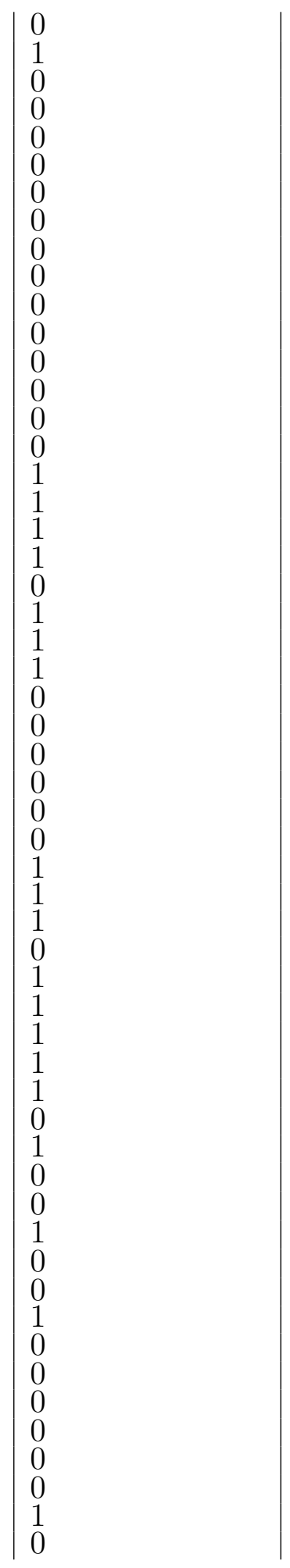




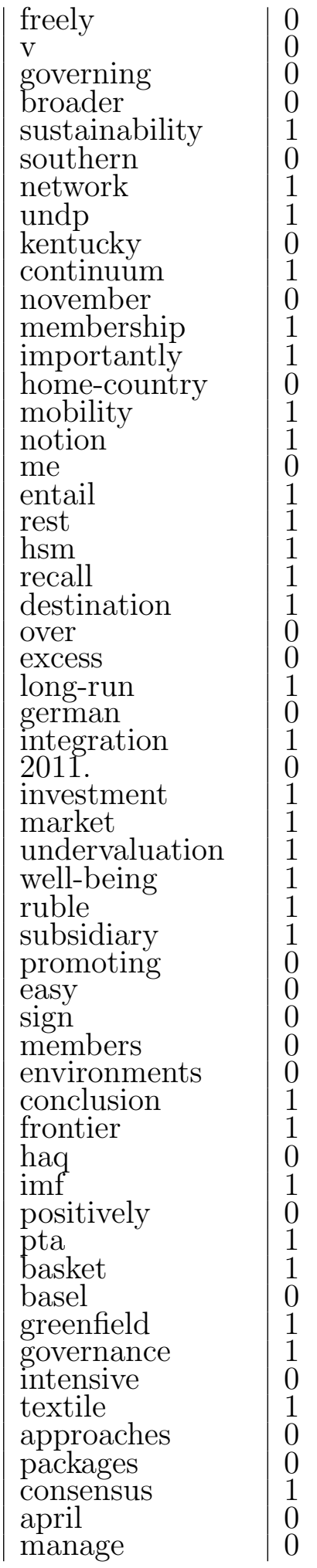

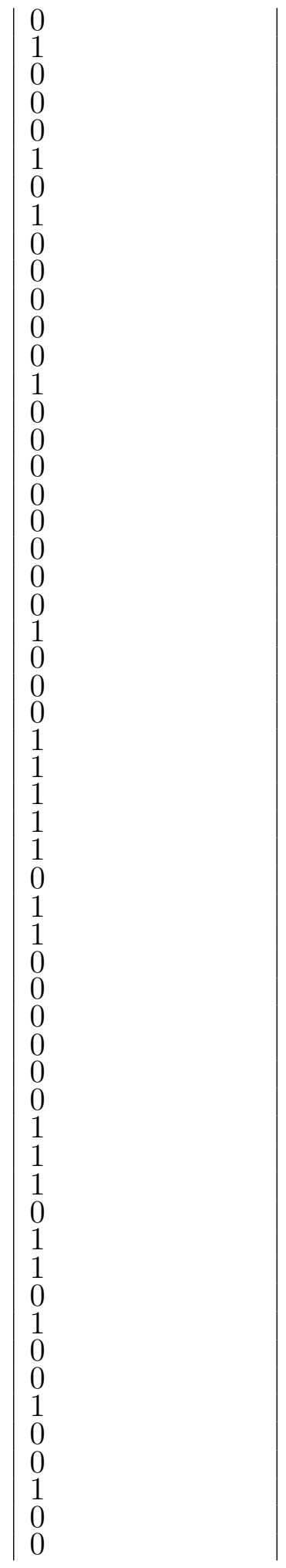




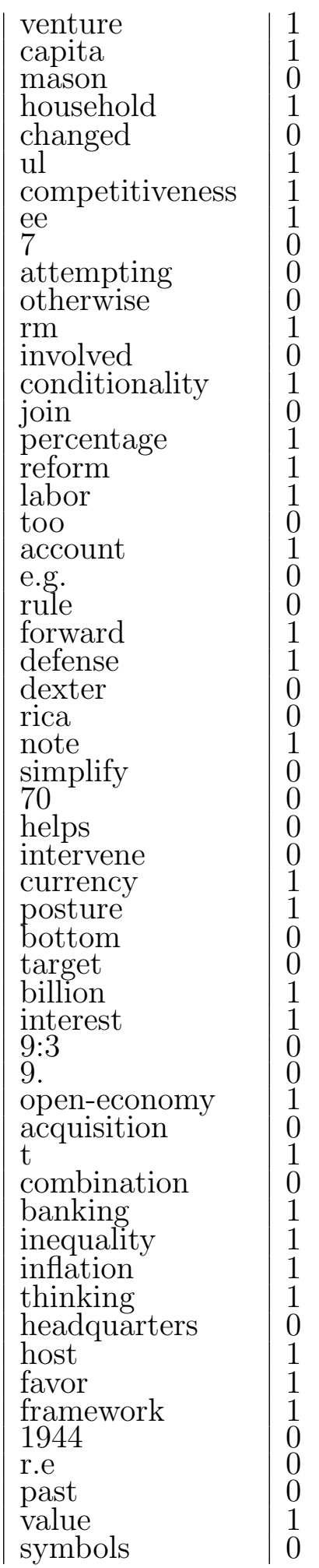

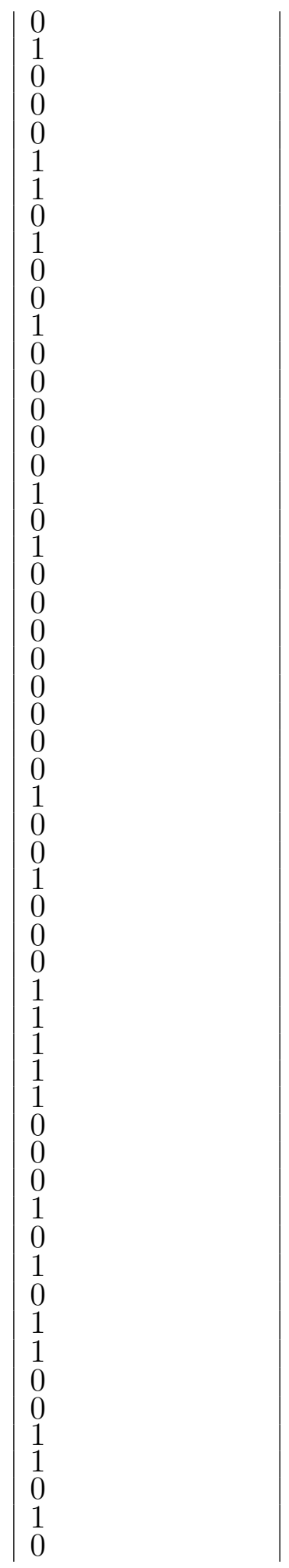




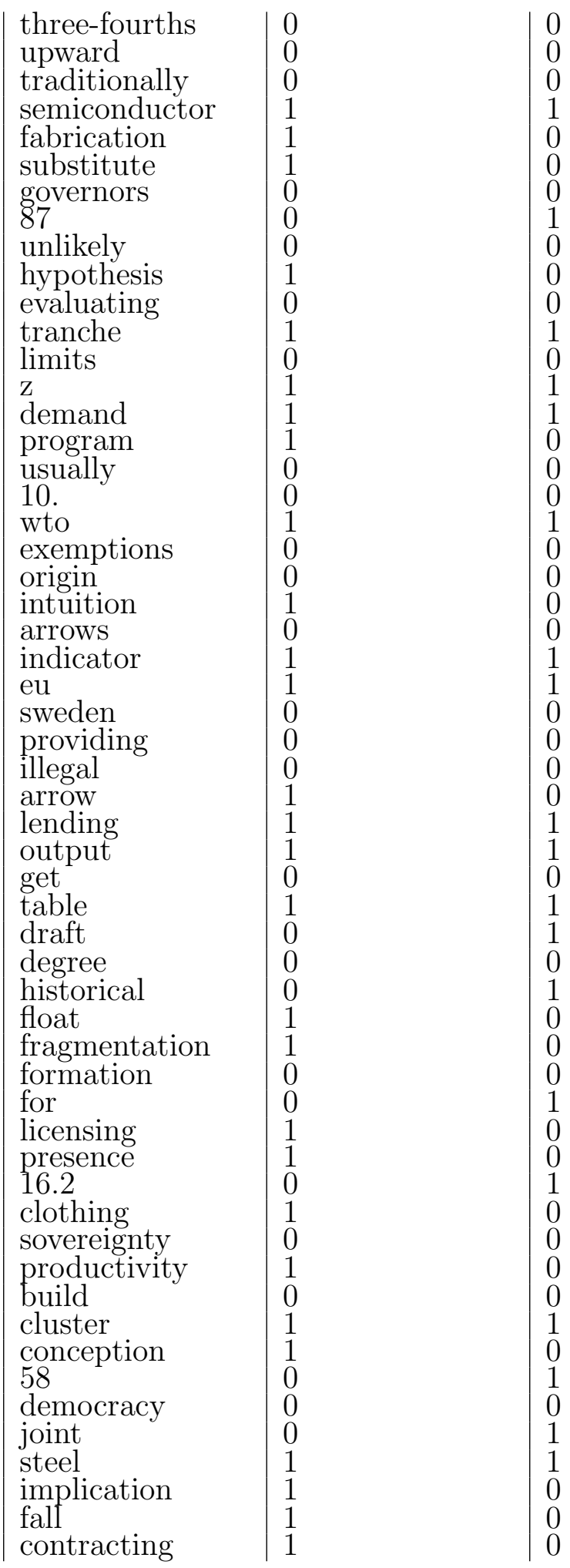




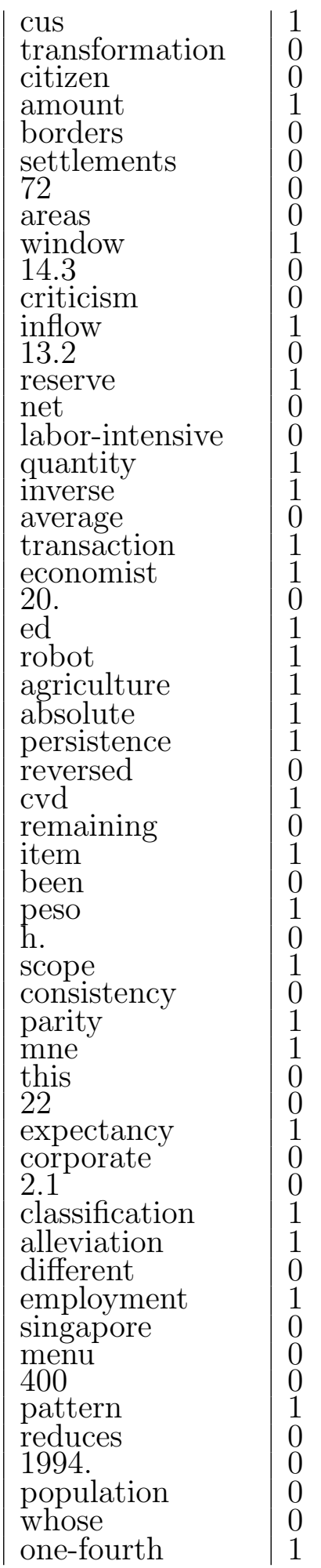

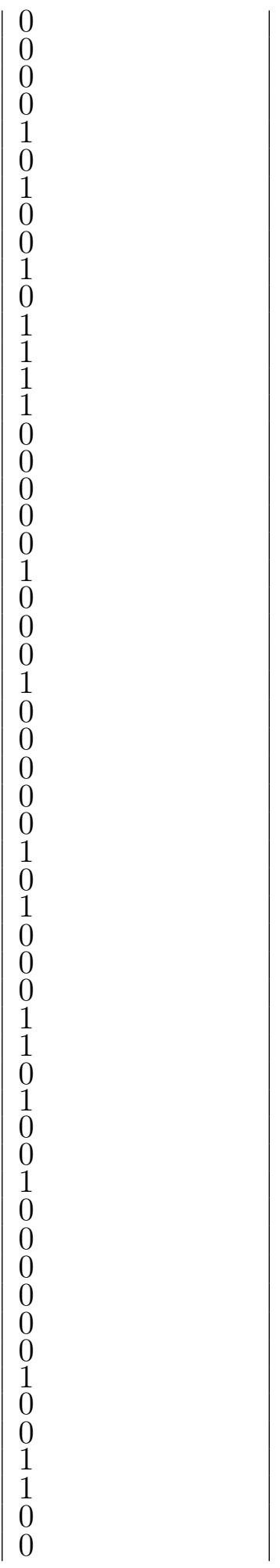




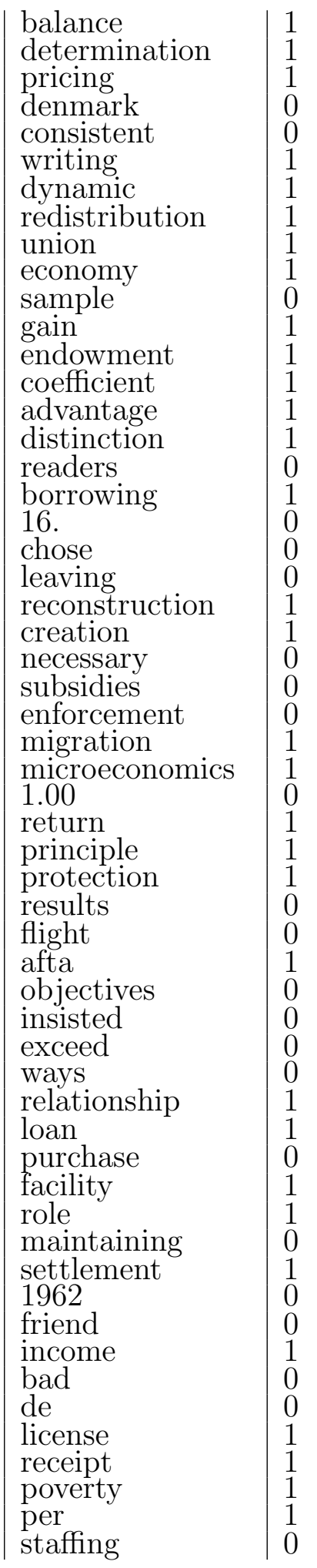

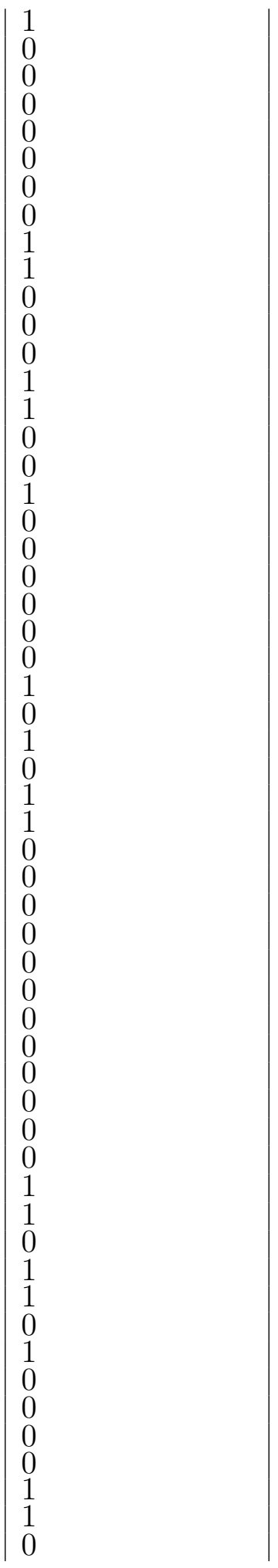




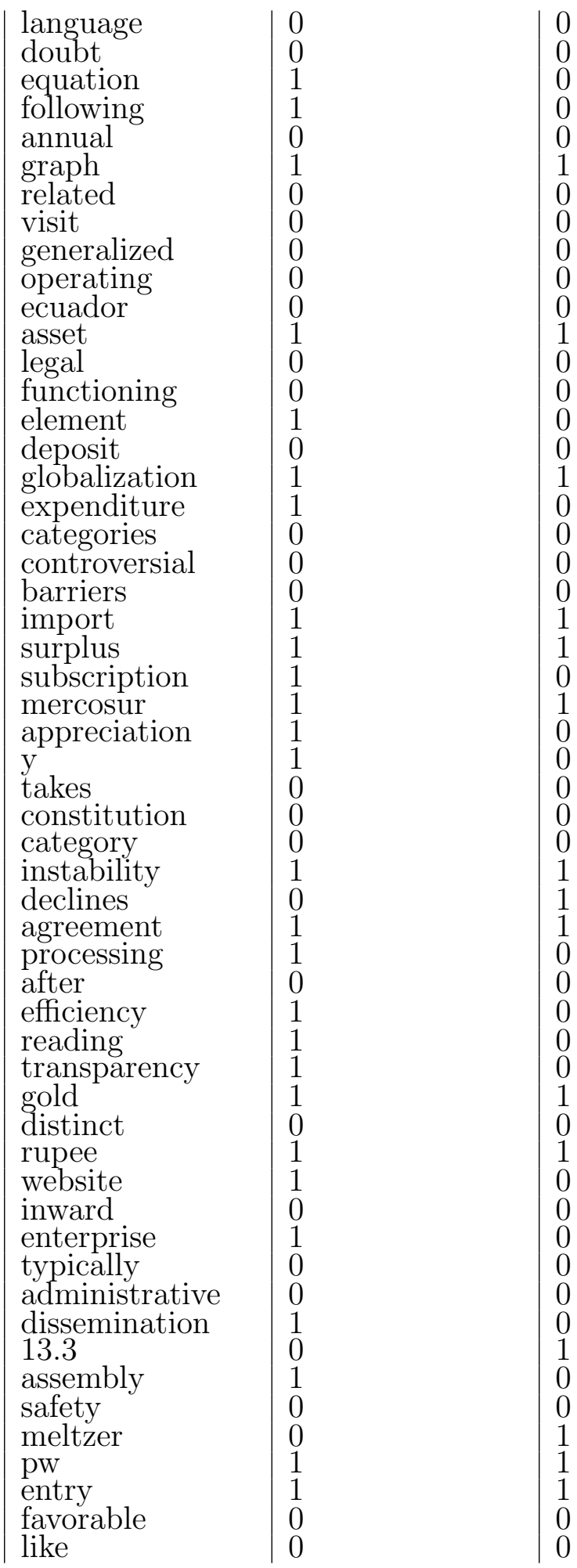




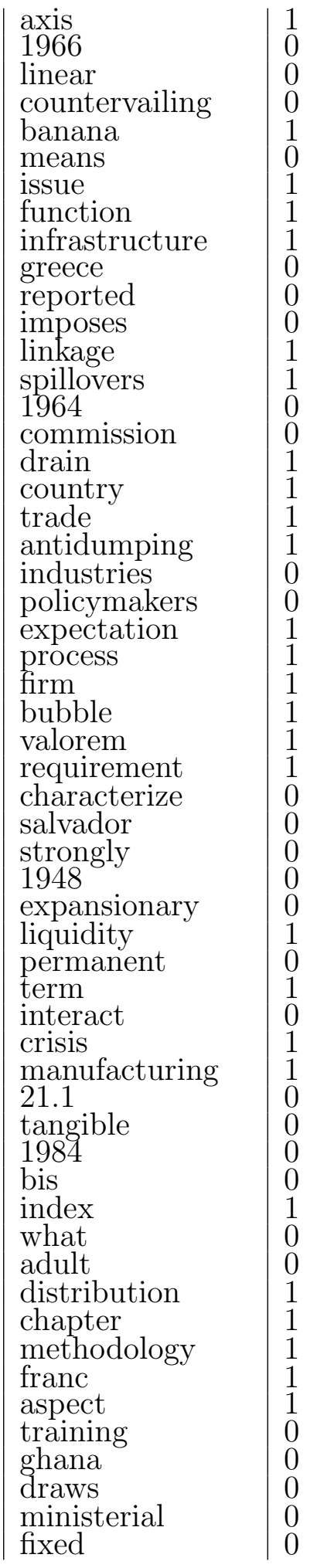

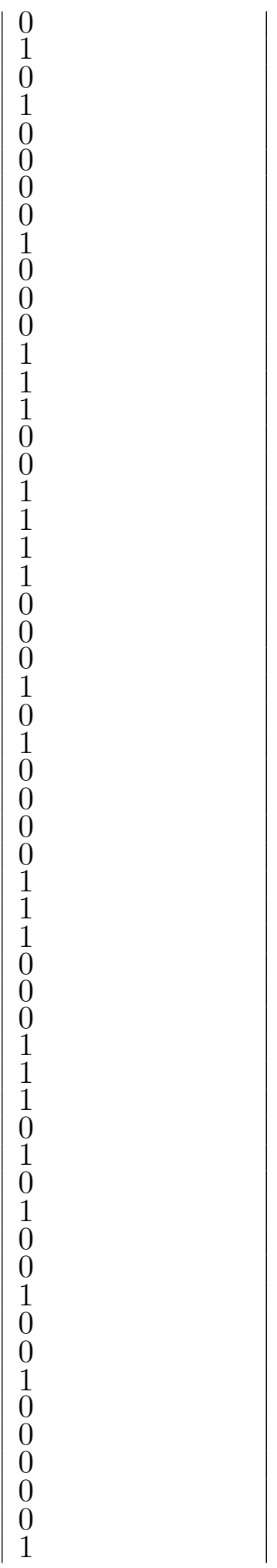




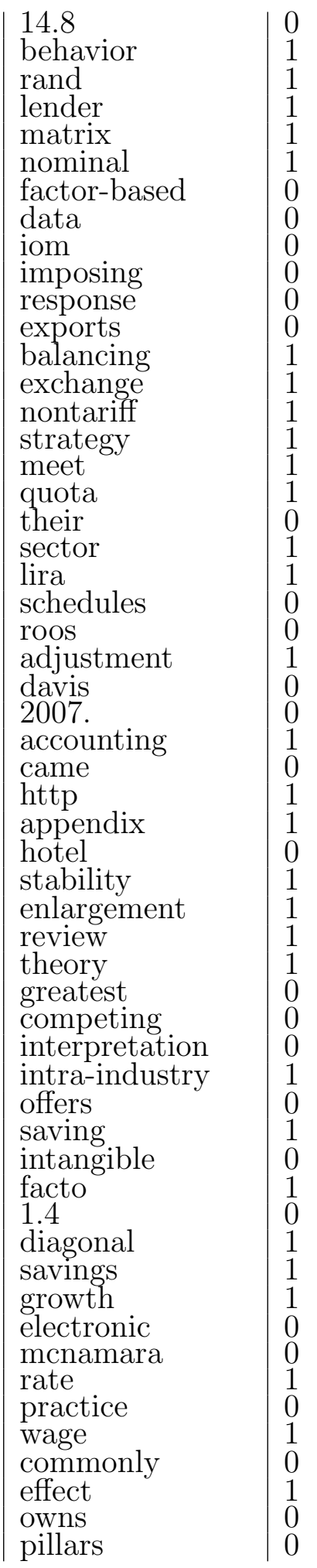

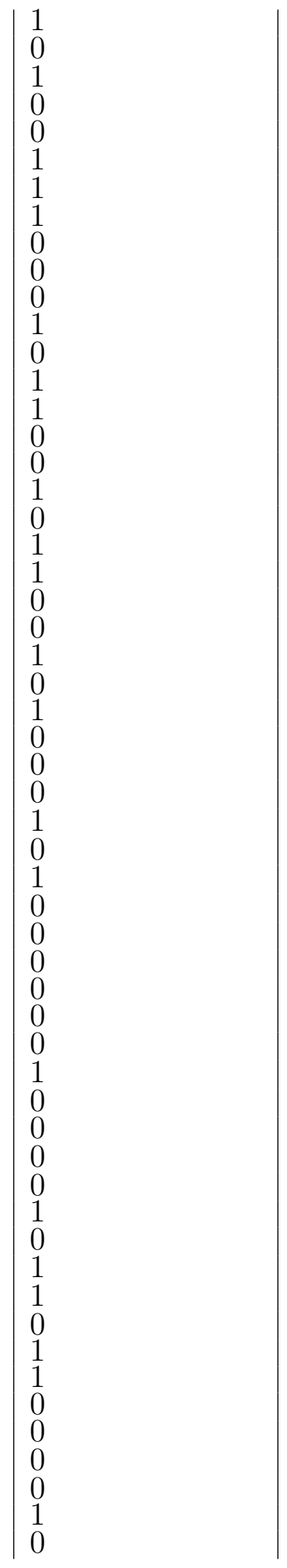




\begin{tabular}{l|l} 
arise & 0 \\
revenue & 1 \\
r. & 0 \\
insight & 1 \\
convergence & 1 \\
measure & 1 \\
korea & 0 \\
strong & 0 \\
factor & 1 \\
deficit & 1 \\
conflict & 0 \\
rise & 1 \\
terminology & 1 \\
budget & 0 \\
euro & 1 \\
1960s & 0 \\
intra-firm & 1 \\
era & 1 \\
side & 0 \\
actions & 0 \\
rus & 1 \\
39 & 0 \\
j & 1 \\
patent & 1 \\
another & 0 \\
adapted & 0 \\
coordination & 1 \\
contagion & 1 \\
dispute & 1 \\
policy & 1 \\
170 & 0 \\
economics & 1 \\
consumption & 1 \\
negotiation & 1 \\
vietnamese & 0 \\
concept & 1 \\
24 & 0 \\
responsibilities & 0 \\
traditional & 0 \\
indicates & 0 \\
milieu & 1 \\
student & 0 \\
10 & 0 \\
triangle & 1 \\
generic & 0 \\
liberalization & 0 \\
es & 0 \\
patents & 0 \\
hice & 0 \\
hyperinflation & 1 \\
organizations & 1 \\
deprivation & 0 \\
brain & 1 \\
shift & 0 \\
smaller &
\end{tabular}

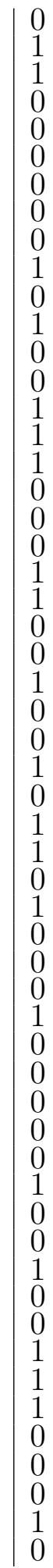




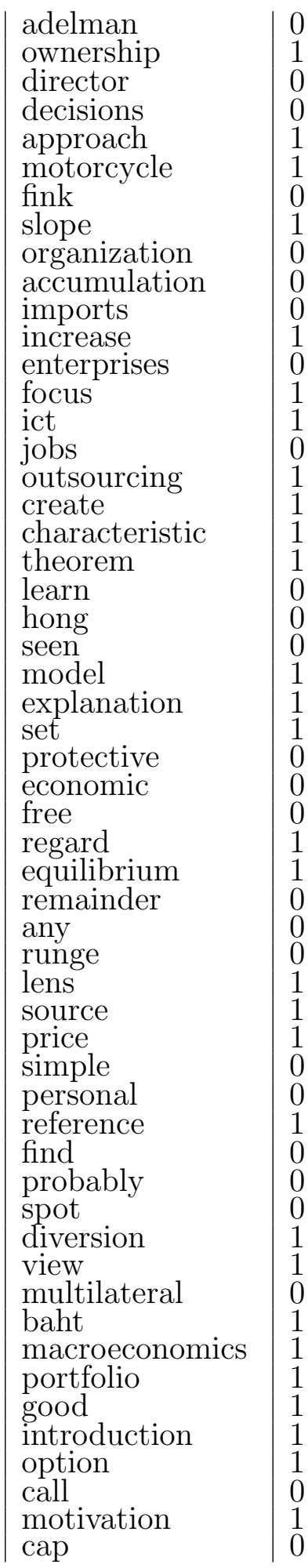
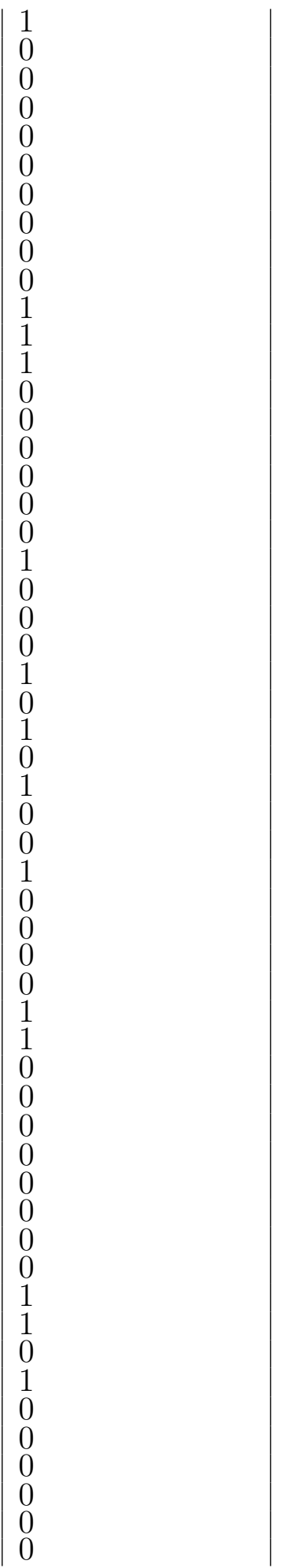


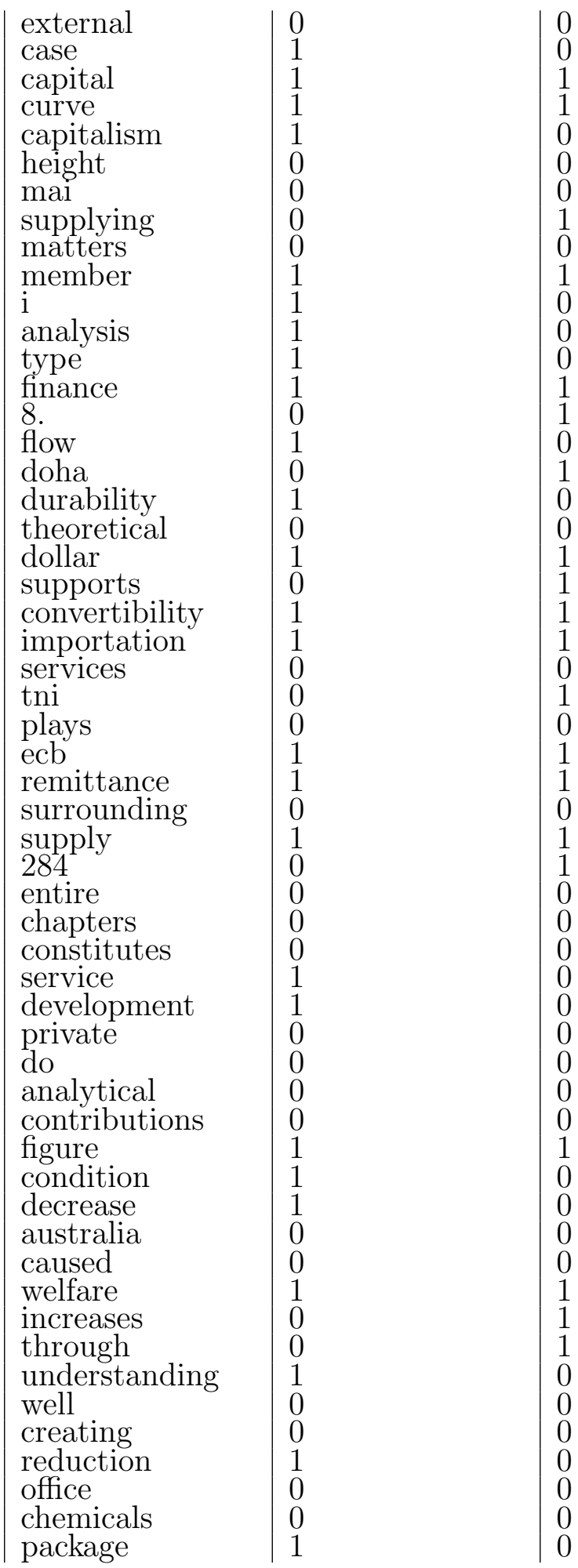

0
0
1
1
0
0
0
1
0
1
0
0
0
1
1
0
1
0
0
1
1
1
1
0
1
0
1
1
0
1
1
0
0
0
0
0
0
0
0
0
1
0
0
0
0
1
1
1
0
0
0
0
0
0
0




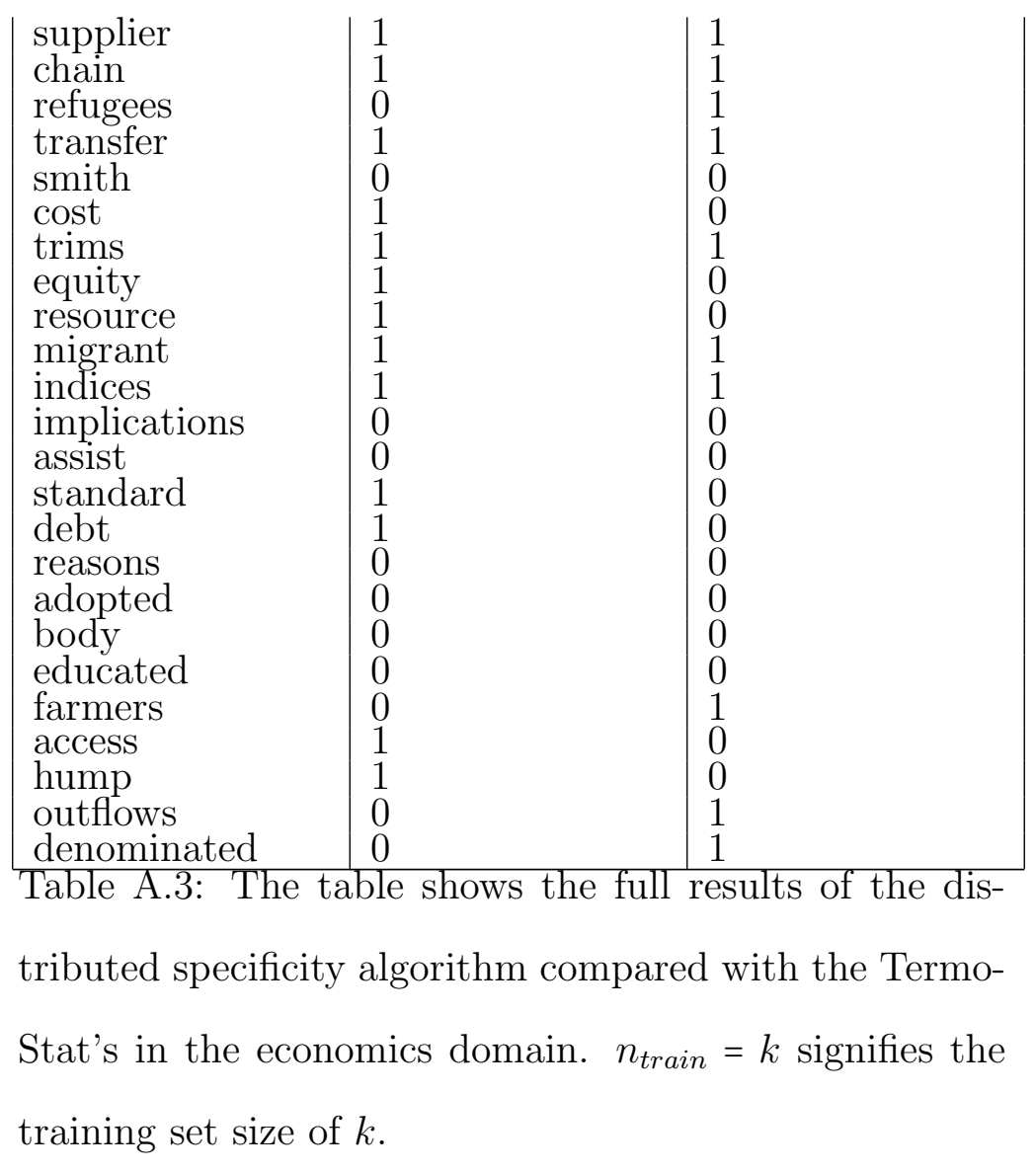

\section{A.4 Politics}

\begin{tabular}{|l|l|l|}
\hline Term Candi- & TermoStat & Distributed \\
date & & Specificity \\
\hline fail & 0 & 0 \\
britain & 0 & 1 \\
ethos & 1 & 1 \\
balance & 1 & 1 \\
opportunities & 0 & 1 \\
result & 1 & 1 \\
slaughter & 1 & 0 \\
corruption & 0 & 1 \\
calculation & 1 & 1 \\
france & 0 & 1 \\
impossible & 0 & 0
\end{tabular}




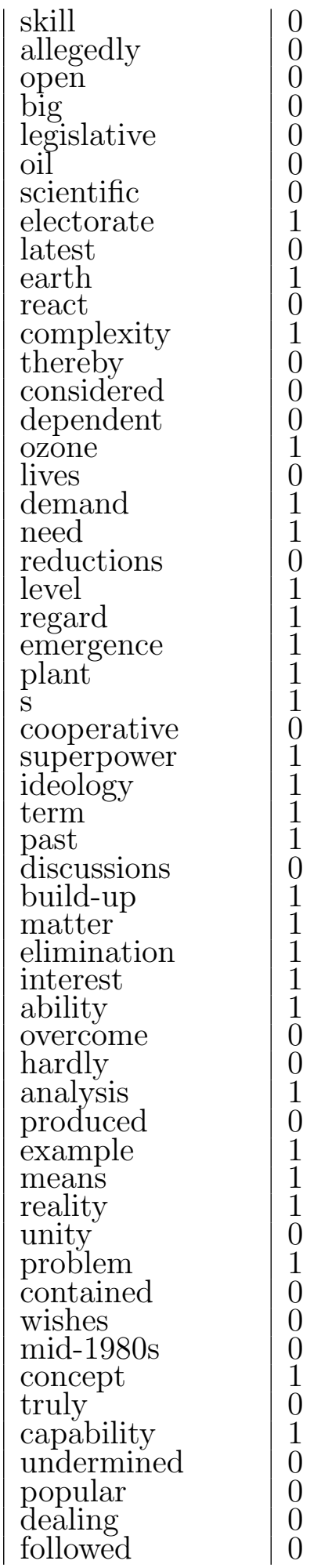

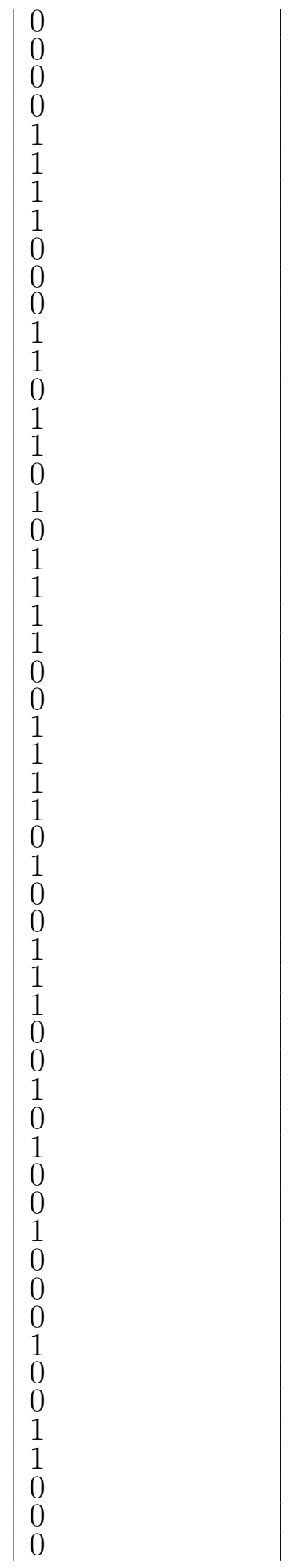




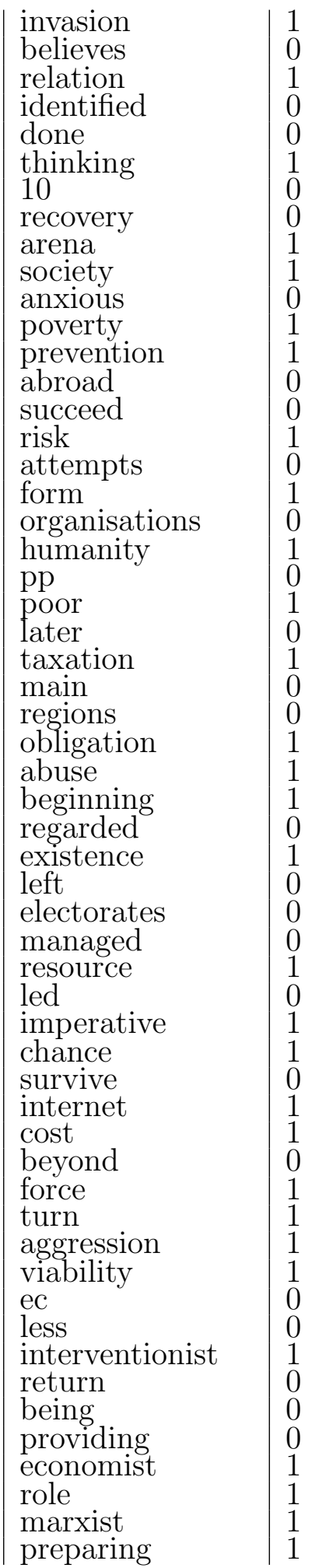
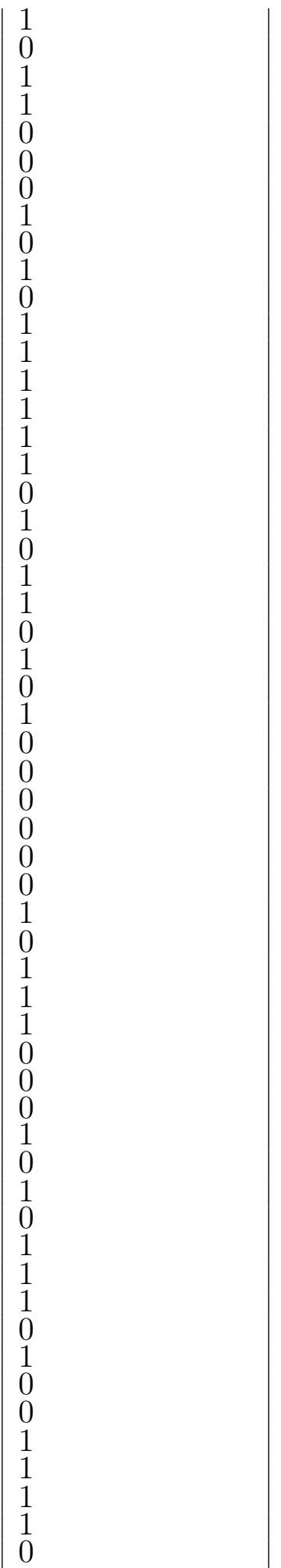


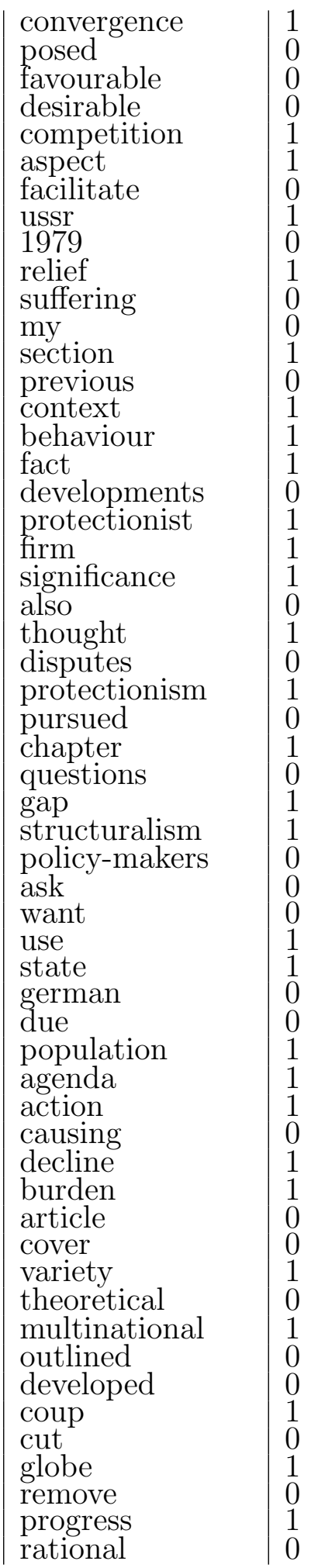

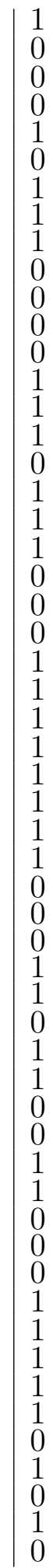




\begin{tabular}{|l|l} 
be & 1 \\
efficient & 0 \\
centres & 0 \\
seats & 0 \\
fellow & 0 \\
dominated & 0 \\
party & 0 \\
mercantilism & 1 \\
strategy & 1 \\
effective & 0 \\
agreed & 0 \\
environment & 1 \\
control & 1 \\
examination & 0 \\
price & 0 \\
failure & 1 \\
fortress & 0 \\
predominantly & 0 \\
currency & 1 \\
limitation & 1 \\
negative & 0 \\
obstruct & 0 \\
things & 0 \\
investment & 1 \\
charter & 0 \\
its & 0 \\
fortune & 1 \\
base & 0 \\
minimum & 0 \\
results & 0 \\
self-interest & 1 \\
fuse & 0 \\
realisation & 1 \\
university & 0 \\
nature & 1 \\
impact & 1 \\
famine & 1 \\
working & 0 \\
unable & 0 \\
apply & 0 \\
attempting & 0 \\
assistance & 0 \\
incentive & 1 \\
commonly & 1 \\
manner & 0 \\
commitment & 1 \\
further & 1 \\
claim & 0 \\
visible & 0 \\
modernisation & 0 \\
wide & 0 \\
independence & 0 \\
leverage & 1 \\
degree & 1 \\
taken & 0 \\
map &
\end{tabular}

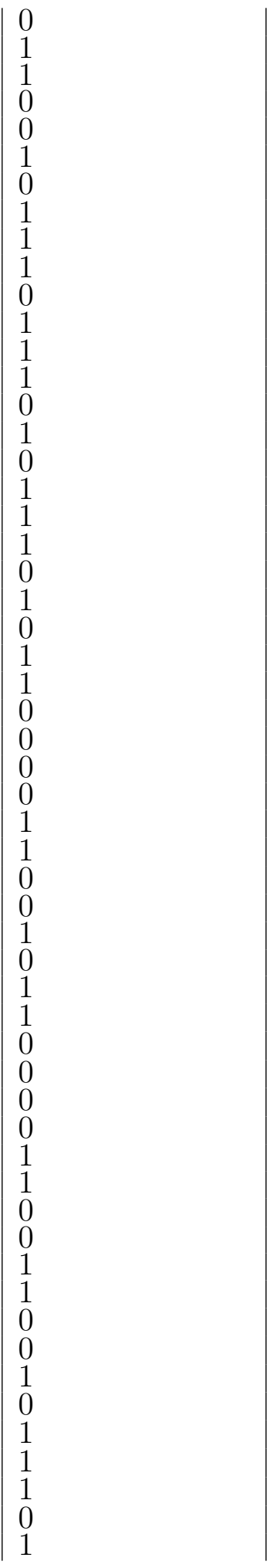




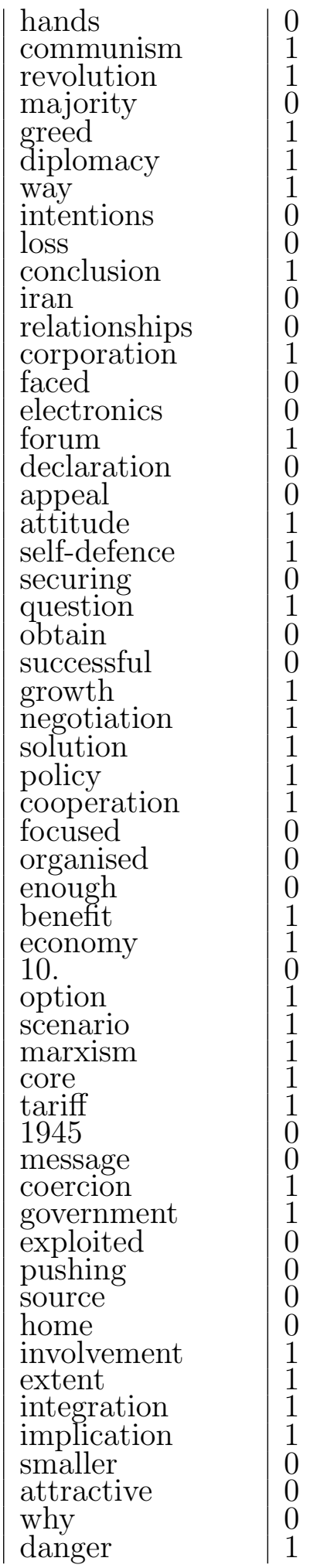

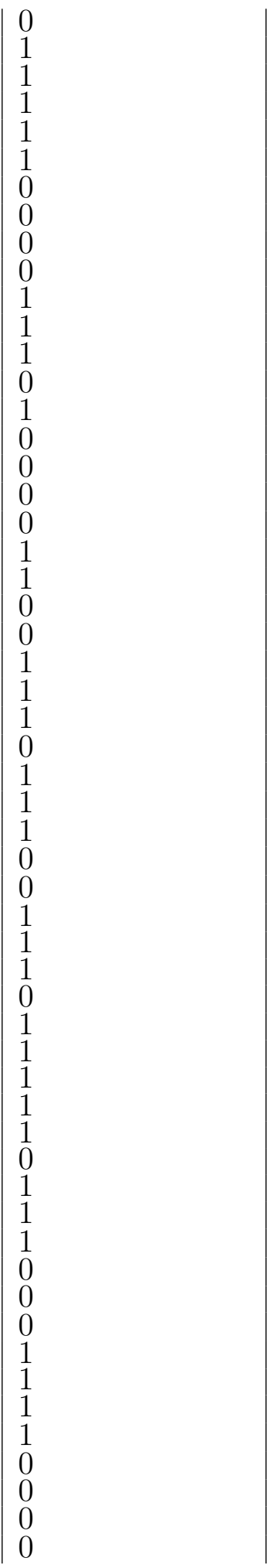




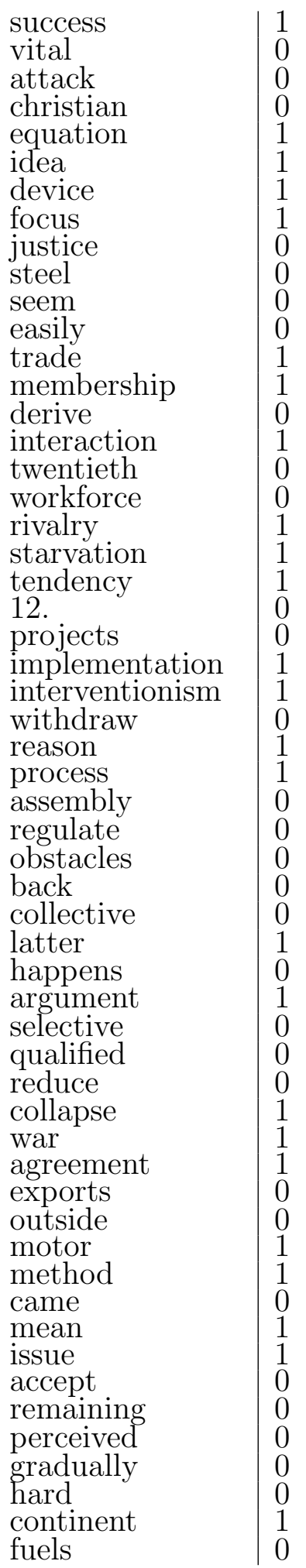

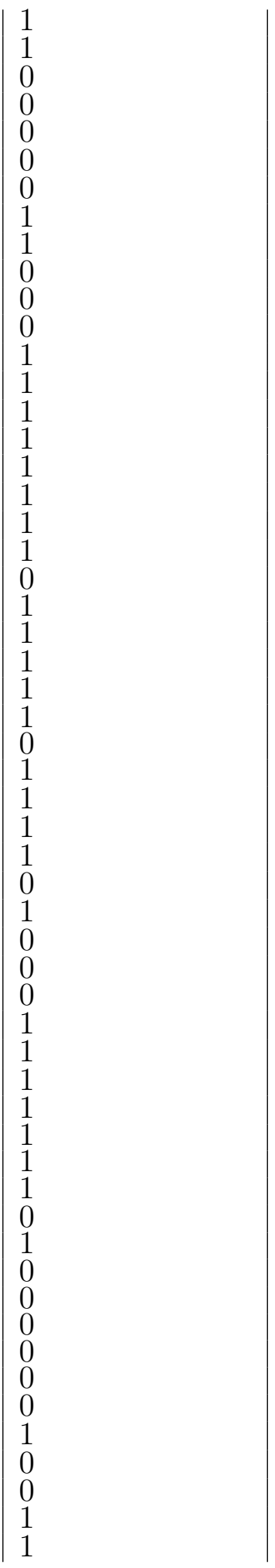




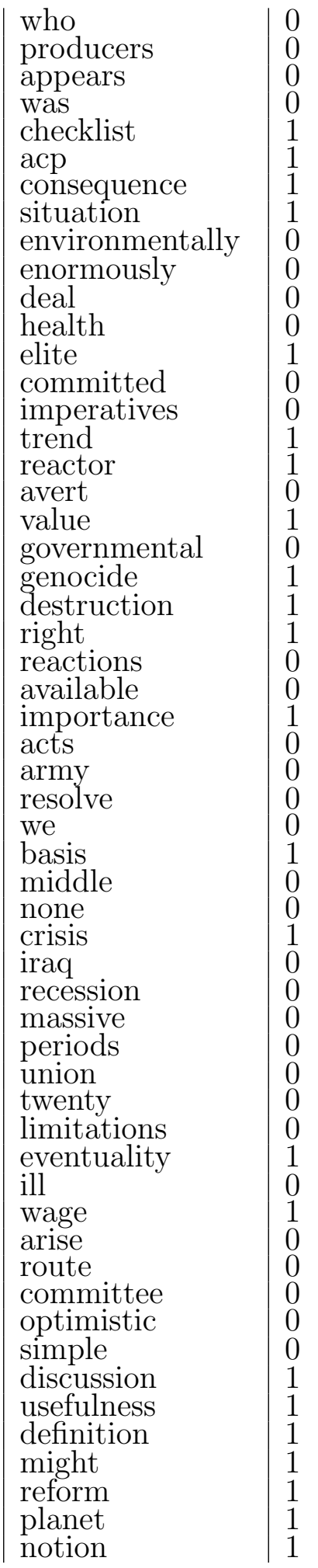

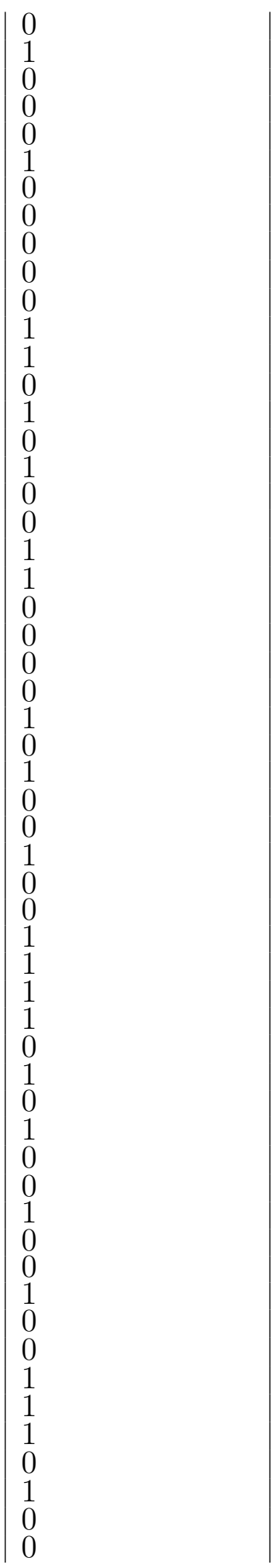




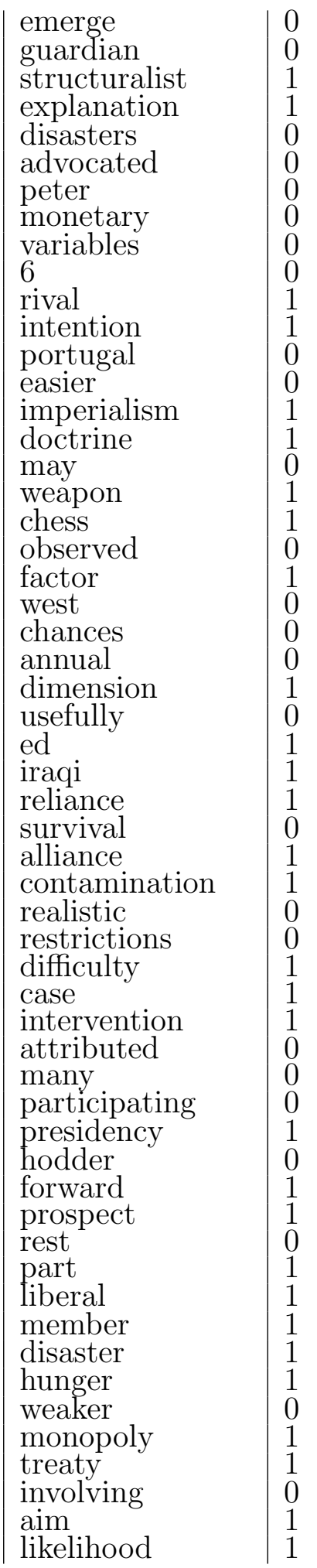

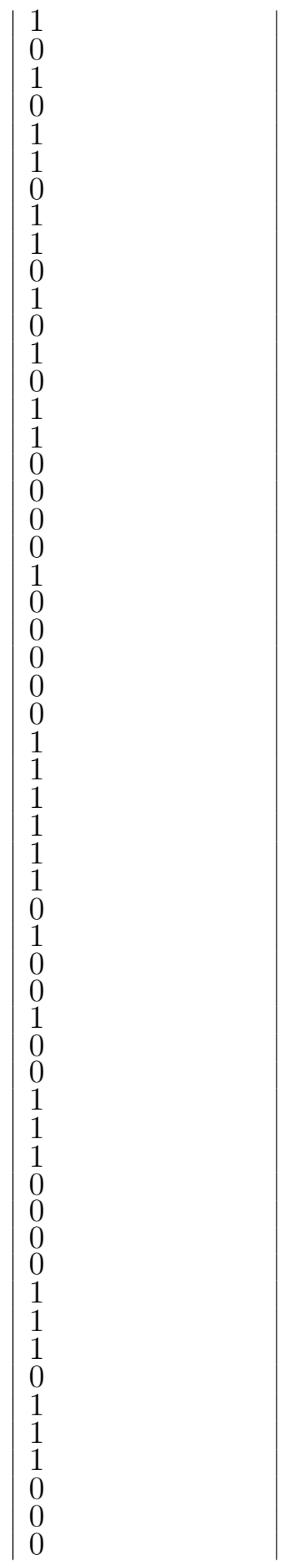




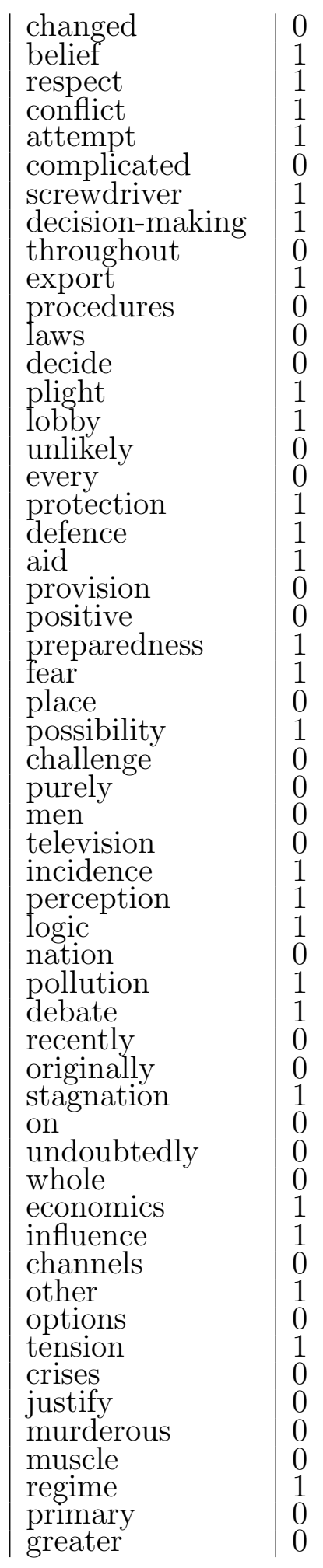

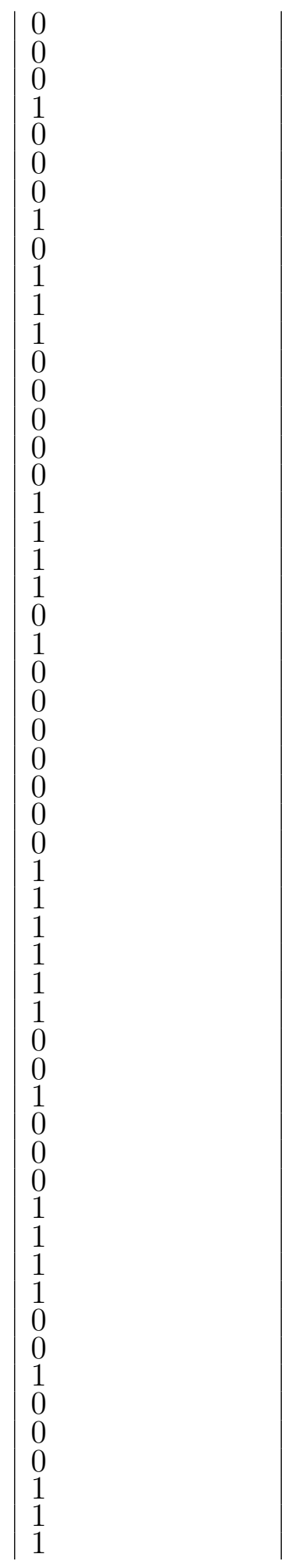




\begin{tabular}{|c|c|c|}
\hline great-power & & 1 \\
\hline continued. & & 0 \\
\hline humanitarian & & 1 \\
\hline $\begin{array}{l}\text { development } \\
\text { future }\end{array}$ & & \\
\hline $\begin{array}{l}\text { tuture } \\
\text { access }\end{array}$ & & \\
\hline alternative & & \\
\hline purpose & & \\
\hline opportunity & & \\
\hline spread & & \\
\hline times & & \\
\hline approach & & \\
\hline known & & \\
\hline transport & & \\
\hline peace & & \\
\hline easy & & \\
\hline $\begin{array}{l}\text { vol } \\
\text { sovereignty }\end{array}$ & & 1 \\
\hline $\begin{array}{l}\text { Soverelgnty } \\
\text { perspective }\end{array}$ & & \\
\hline position & & \\
\hline capitalism & & \\
\hline framework & & \\
\hline scale & & 1 \\
\hline good & & \\
\hline $\begin{array}{l}\text { well-being } \\
\text { number }\end{array}$ & & \\
\hline combination & & \\
\hline potential & & \\
\hline threat & & \\
\hline pursuit. & & \\
\hline humankind & & \\
\hline growing & & \\
\hline security & & \\
\hline $\begin{array}{l}\text { organisation } \\
\text { nato }\end{array}$ & & \\
\hline broke & & \\
\hline namely & & \\
\hline india & & \\
\hline prediction & & \\
\hline $\begin{array}{l}9 . \\
\text { sense }\end{array}$ & & \\
\hline $\begin{array}{l}\text { sense } \\
\text { individual }\end{array}$ & & \\
\hline permission & & \\
\hline relevance & & \\
\hline veto & & \\
\hline often & & \\
\hline market & & \\
\hline capitalist & & \\
\hline taiwan & & \\
\hline room & & \\
\hline containment & & \\
\hline $\begin{array}{l}\text { thing } \\
\text { svstem }\end{array}$ & & \\
\hline $\begin{array}{l}\text { system } \\
\text { wealth }\end{array}$ & & \\
\hline world & & \\
\hline 1993 & & \\
\hline
\end{tabular}

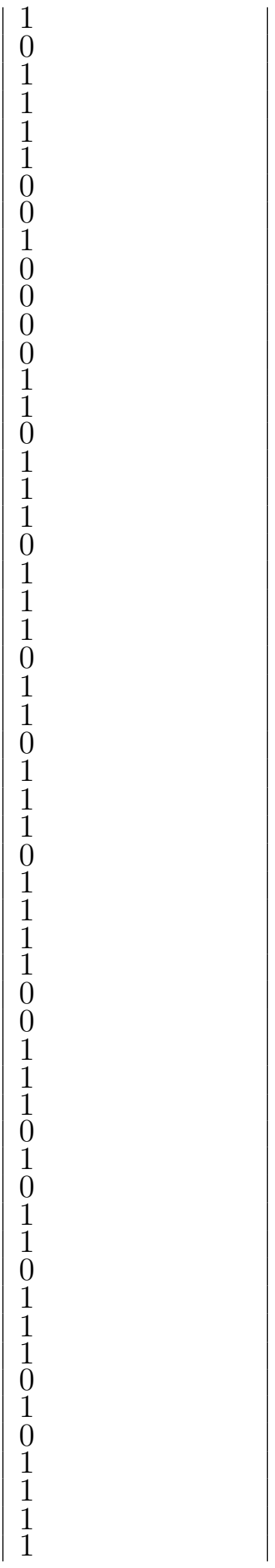




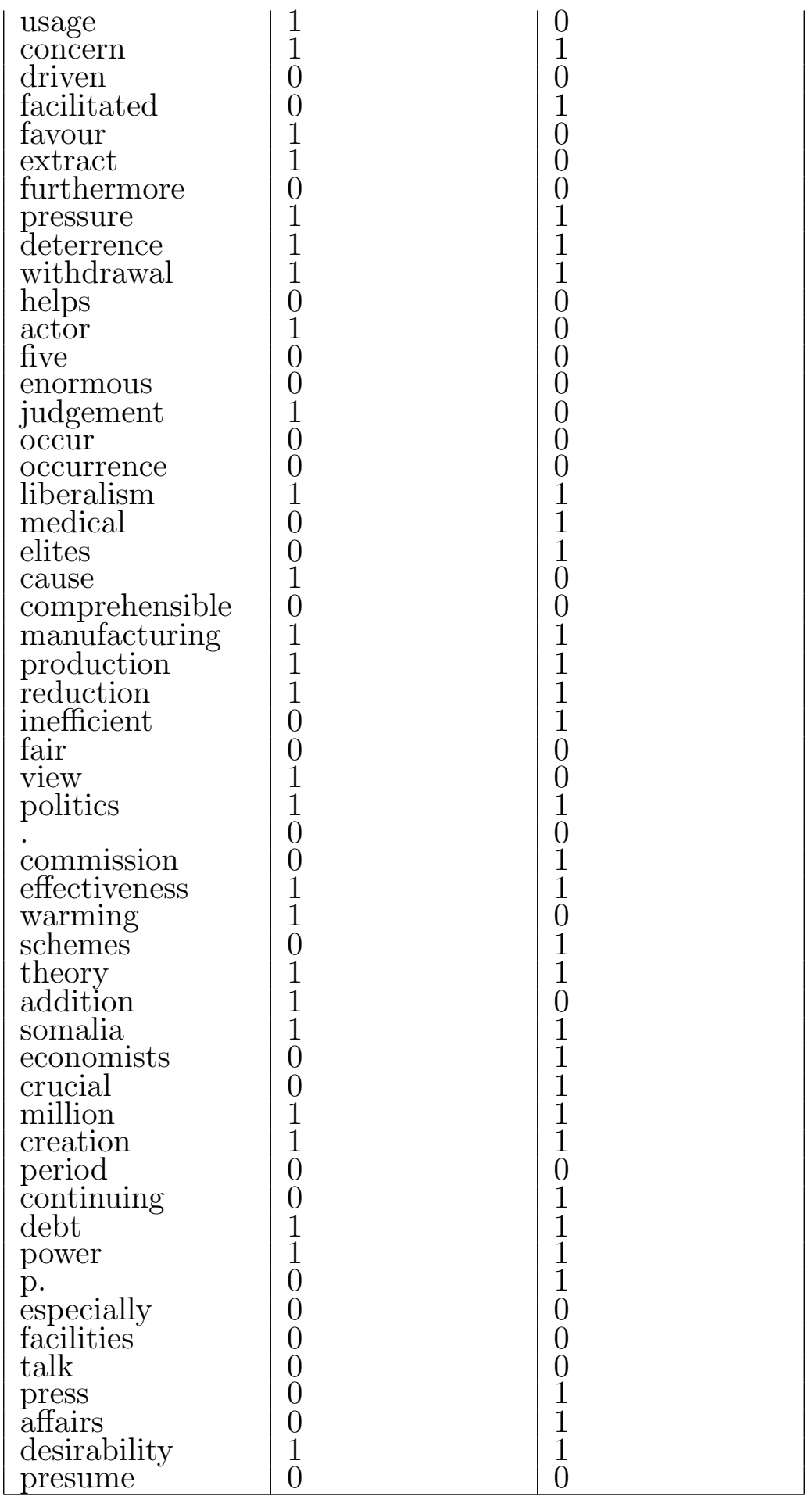


Table A.4: The table shows the full results of the distributed specificity algorithm compared with the TermoStat's in the politics domain. $n_{\text {train }}=k$ signifies the training set size of $k$. 


\section{Appendix B}

\section{List of Stop Words Used in}

\section{Domain Adaptation Experiments}

\section{as Anchor Points}

\begin{tabular}{|l|l|l|l|}
\hline Stop Word & Stop Word & Stop Word & Stop Word \\
\hline being & - & - & all \\
had & shrough & its & before \\
has & should & to & only \\
very & then & them & his \\
did & they & not & during \\
where & because & she & each \\
further & out & what & are \\
while & does & above & for \\
be & we & after & between \\
by & both & about & here \\
of & could & against & her \\
own & yourself & down & or \\
from & would & their & your \\
been & few & too & was \\
until & more & that & but \\
with & than & those & he \\
me & these & below & were \\
my & and & do & is \\
am & it & an & as \\
itself & at & have & in \\
\hline
\end{tabular}




\begin{tabular}{|l|l|l|l|} 
any & if & again & no \\
when & same & how & other \\
which & you & who & most \\
such & why & a & i \\
so & the & having & once \\
\hline
\end{tabular}

Table B.1: The table shows the list of stop words used

in the dissertation as anchor points. 


\section{Appendix $\mathrm{C}$}

\section{List of Stop Words Used in}

\section{Chapter 3}

\begin{tabular}{|l||l|l|l|}
\hline Stop Word & Stop Word & Stop Word & Stop Word \\
\hline- & - & - & all \\
she'll & don't & being & the \\
through & yourselves & its & before \\
he's & when's & we've & had \\
should & he'd & to & only \\
there's & those & who & has \\
haven't & do & them & his \\
they'll & very & who's & it \\
they'd & cannot & you've & they \\
not & during & yourself & him \\
nor & we'll & did & they've \\
this & she & each & won't \\
where & mustn't & isn't & i'll \\
why's & because & you'd & doing \\
some & as & are & further \\
ourselves & or & out & what \\
for & while & wasn't & does \\
shouldn't & above & between & is \\
be & we & who & you're \\
were & here & hers & aren't \\
by & both & about & would \\
of & could & against & i'd \\
weren't & i'm & or & can't \\
own & for & whom & down \\
hadn't & couldn't & your & doesn't \\
& &
\end{tabular}




\begin{tabular}{|l||l|l|l|} 
from & how's & her & their \\
it's & be & there & been \\
why & until & too & themselves \\
was & more & himself \\
where's & but & with & didn't \\
what's & what & was \\
herself & than & here's & he \\
me & hasn't & myself & are \\
these & below & by \\
ought & we'd & to & my \\
wouldn't & amd & then \\
is & itself & it & an \\
as & any & at & have \\
in & no & if & again \\
of & how & other & when \\
same & you & which \\
I & an & shan't & on \\
a & our & after \\
let's & he'll & ours & such \\
in & she'd & a & at \\
i & so & yours & you'll \\
about & that's & we're & she's \\
the & having & once \\
\hline
\end{tabular}

Table C.1: The table shows the full list of stop words

used in the dissertation as filters in chapter 3 . 


\section{Bibliography}

Abid, M. R., Petriu, E. M., and Amjadian, E. (2015). Dynamic sign language recognition for smart home interactive application using stochastic linear formal grammar. IEEE Trans. Instrumentation and Measurement, 64(3):596-605.

Amjadian, E., Inkpen, D., Paribakht, T., and Faez, F. (2016). Local-global vectors to improve unigram terminology extraction. In Proceedings of the 5th International Workshop on Computational Terminology (Computerm2016), pages 2-11, Osaka, Japan. The COLING 2016 Organizing Committee.

Amjadian, E., Inkpen, D., Paribakht, T. S., and Faez, F. (2018). Distributed specificity for automatic terminology extraction. Terminology, 24(1):23-40.

Anderson, P. (2005). The Global Politics of Power, Justice and Death: An Introduction to International Relations. Taylor \& Francis.

Anthony, L. (2012). AntConc (Version 3.3.0) [Computer Software]. Tokyo, Japan: Waseda University. Available from http://www.laurenceanthony.net/.

Anvari, A. (2014). Some remarks on bridging. Honours thesis, Carleton University. 
Apache Software Foundation (2014). openNLP Natural Language Processing Library. http://opennlp.apache.org/.

Asher, N. and Lascarides, A. (1998). Bridging. Journal of Semantics, 15(1):83-113.

Beaver, D. and Zeevat, H. (2012). Accommodation. In Ramchand, G. and Reiss, C., editors, Oxford Handbook of Linguistic Interfaces. Oxford University Press, Oxford.

Bengio, S. and Heigold, G. (2014). Word embeddings for speech recognition. In Proceedings of the 15th Conference of the International Speech Communication Association, Interspeech.

Bengio, Y., Ducharme, R., Vincent, P., and Janvin, C. (2003). A neural probabilistic language model. J. Mach. Learn. Res., 3:1137-1155.

Betsy, T. and Keogh, J. (2012). Microbiology DeMYSTiFieD, 2nd Edition. Demystified. McGraw-Hill Education.

Birte U. Forstmann, E.-J. W. e. (2015). An Introduction to Model-Based Cognitive Neuroscience. Springer-Verlag New York, 1 edition.

Blei, D. M. (2012). Probabilistic topic models. Commun. ACM, 55(4):77-84.

Blei, D. M., Ng, A. Y., and Jordan, M. I. (2003). Latent dirichlet allocation. J. Mach. Learn. Res., 3:993-1022.

Bojanowski, P., Grave, E., Joulin, A., and Mikolov, T. (2016). Enriching word vectors with subword information. arXiv preprint arXiv:1607.04606. 
Brill, E. (1992). A simple rule-based part of speech tagger. In Proceedings of the Third Conference on Applied Natural Language Processing, ANLC '92, pages 152155, Stroudsburg, PA, USA. Association for Computational Linguistics.

Broß, J. and Ehrig, H. (2013). Terminology extraction approaches for product aspect detection in customer reviews. In Hockenmaier, J. and Riedel, S., editors, Proceedings of the Seventeenth Conference on Computational Natural Language Learning, CoNLL 2013, Sofia, Bulgaria, August 8-9, 2013, pages 222-230. ACL.

Brown, P. F., deSouza, P. V., Mercer, R. L., Pietra, V. J. D., and Lai, J. C. (1992). Class-based n-gram models of natural language. Comput. Linguist., 18(4):467-479.

Cabre-Castellvi, M. T., Bagot, R. E., and Vivaldi-Palatresi, J. (2001). Automatic term detection: A review of current systems. In Bourigault, D., Jacquemin, C., and LHomme, M., editors, Recent Advances in Computational Terminology. John Benjamins.

Cahill, A. and Riester, A. (2012). Automatically acquiring fine-grained information status distinctions in german. In Proceedings of the SIGDIAL 2012 Conference, The 13th Annual Meeting of the Special Interest Group on Discourse and Dialogue, 5-6 July 2012, Seoul National University, Seoul, South Korea, pages 232-236. The Association for Computer Linguistics.

Carrillo, H., Brodersen, K. H., and Castellanos, J. A. (2014). Probabilistic Performance Evaluation for Multiclass Classification Using the Posterior Balanced Accuracy, pages 347-361. Springer International Publishing, Cham.

Castellví, M. T. C., Bagot, R. E., and Palatresi, J. V. (2001). Automatic term detec- 
tion: A review of current systems. Recent advances in computational terminology, $2: 53-88$.

Chierchia, G. (1995). Dynamics of meaning: Anaphora, presupposition, and the theory of grammar. University of Chicago Press, Chicago, IL.

Choi, H., Cho, K., and Bengio, Y. (2018). Fine-grained attention mechanism for neural machine translation. Neurocomputing, 284:171-176.

Chomsky, N. (1956). Three models for the description of language. IRE Transactions on Information Theory, 2:113-124. http://www.chomsky.info/articles/ $195609--. p d f-$ last visited $14^{\text {th }}$ January 2009.

Chomsky, N. (1957). Syntactic Structures. Mouton and Co., The Hague.

Chung, T. M. (2003). A corpus comparison approach for terminology extraction. Terminology, 9:221-246.

Chung, T. M. and Nation, P. (2004). Identifying technical vocabulary. System, $32(2): 251-263$.

Clark, H. H. (1975). Bridging. In Proceedings of the 1975 Workshop on Theoretical Issues in Natural Language Processing, TINLAP '75, pages 169-174, Stroudsburg, PA, USA. Association for Computational Linguistics.

Collobert, R. and Weston, J. (2008). A unified architecture for natural language processing: Deep neural networks with multitask learning. In Proceedings of the 25th International Conference on Machine Learning, ICML '08, pages 160-167, New York, NY, USA. ACM. 
Collobert, R., Weston, J., Bottou, L., Karlen, M., Kavukcuoglu, K., and Kuksa, P. P. (2011). Natural language processing (almost) from scratch. CoRR, abs/1103.0398.

Conneau, A., Lample, G., Ranzato, M., Denoyer, L., and Jégou, H. (2017). Word translation without parallel data. CoRR, abs/1710.04087.

Conrado, M., Pardo, T., and Rezende, S. (2013). A machine learning approach to automatic term extraction using a rich feature set. page 16-23, Atlanta, Georgia. Association for Computational Linguistics.

Crippin, P., Donato, R., and Wright, D. Calculus and Vectors. Toronto, Ontario.

Csurka, G. (2017). Domain adaptation for visual applications: A comprehensive survey. Advances in Computer Vision and Pattern Recognition, pages "1-35".

de Saussure, F. ([1916] 1983). Course in General Linguistics. Duckworth, London. (trans. Roy Harris).

Deerwester, S., Dumais, S. T., Furnas, G. W., Landauer, T. K., and Harshman, R. (1990). Indexing by latent semantic analysis. JOURNAL OF THE AMERICAN SOCIETY FOR INFORMATION SCIENCE, 41(6):391-407.

Dinu, G. and Baroni, M. (2014). Improving zero-shot learning by mitigating the hubness problem. CoRR, abs/1412.6568.

Domingos, P. and Lowd, D. (2009). Markov Logic: An Interface Layer for Artificial Intelligence. Morgan and Claypool Publishers, 1st edition.

Drouin, P. (2003). Term extraction using non-technical corpora as a point of leverage. Terminology, 9(1):99-115. 
Duchi, J., Hazan, E., and Singer, Y. (2011). Adaptive subgradient methods for online learning and stochastic optimization. J. Mach. Learn. Res., 12:2121-2159.

Durrett, G. and Klein, D. (2013). Easy victories and uphill battles in coreference resolution. In Proceedings of the 2013 Conference on Empirical Methods in Natural Language Processing, pages 1971-1982, Seattle, Washington, USA. Association for Computational Linguistics.

Elliott, D. (2013). Clinical Procedures in Primary Eye Care: Expert Consult: Online and Print. Elsevier Health Sciences.

Elman, J. L. (1990). Finding structure in time. Cognitive Science, 14(2):179-211.

Emami, A. and Jelinek, F. (2004). Exact training of a neural syntactic language model. In 2004 IEEE International Conference on Acoustics, Speech, and Signal Processing, ICASSP 2004, Montreal, Quebec, Canada, May 17-21, 2004, pages $245-248$.

Enguehard, C. (2003). Correct: Demarche cooperative pour l'evaluation de systemes de reconnaissance de termes. In In Actes de la 10eme conference annuelle sur le Traitement Automatique des Langues (TALN 2003), pages 339-345, Nancy, France.

Fedorenko, D., Astrakhantsev, N., and Turdakov, D. (2014). Automatic recognition of domain-specific terms: an experimental evaluation. Proceedings of the Institute for System Programming of RAS, 26:55-72.

Fellbaum, C., editor (1998). WordNet: An Electronic Lexical Database. Language, Speech, and Communication. MIT Press, Cambridge, MA. 
Feuerbach, T., Riedl, M., and Biemann, C. (2015). Distributional semantics for resolving bridging mentions. In Recent Advances in Natural Language Processing, RANLP 2015, 7-9 September, 2015, Hissar, Bulgaria, pages 192-199.

Firth, J. R. (1957). A synopsis of linguistic theory 1930-55. 1952-59:1-32.

Frantzi, K., Ananiadou, S., and Mima, H. (2000). Automatic recognition of multiword terms: the c-value/nc-value method. International Journal on Digital Libraries, $3(2): 115-130$.

Frantzi, K. T., Ananiadou, S., and Tsujii, J.-i. (1998). The c-value/nc-value method of automatic recognition for multi-word terms. In Proceedings of the Second European Conference on Research and Advanced Technology for Digital Libraries, ECDL '98, pages 585-604, London, UK, UK. Springer-Verlag.

Gharbieh, W., Bhavsar, V., and Cook, P. (2017). Deep Learning Models For Multiword Expression Identification. In Proceedings of the 6th Joint Conference on Lexical and Computational Semantics ( ${ }^{*}$ SEM 2017), pages 54-64.

Griffiths, T. L., Steyvers, M., Blei, D. M., and Tenenbaum, J. B. (2005). Integrating topics and syntax. In In Advances in Neural Information Processing Systems 17, pages 537-544. MIT Press.

Hadi, W. M. E., Timimi, I., Dabbadie, M., Choukri, K., Hamon, O., and Chiao, Y. (2006). Terminological resources acquisition tools: Toward a user-oriented evaluation model. In Proceedings of the Fifth International Conference on Language Resources and Evaluation, LREC 2006, Genoa, Italy, May 22-28, 2006., pages 945-948. 
Haghighi, A., Liang, P., Berg-Kirkpatrick, T., and Klein, D. (2008). Learning bilingual lexicons from monolingual corpora. In Proceedings of $A C L-08$ : HLT, pages 771-779. Association for Computational Linguistics.

Hall, M., Frank, E., Holmes, G., Pfahringer, B., Reutemann, P., and Witten, I. H. (2009). The weka data mining software: An update. SIGKDD Explor. Newsl., 11(1):10-18.

Harris, Z. (1954). Distributional structure. Word, 10(23):146-162.

Hawkins, J. A. (1978). Definiteness and Indefiniteness: a study in reference and grammaticality prediction. Croom Helm.

Heim, I. (1982). On the semantics of definite and indefinite noun phrases. Doctoral Dissertation, University of Massachusetts at Amherst.

Heim, I. and Kratzer, A. (1998). Semantics in Generative Grammar. Blackwell, Oxford.

Hinton, G. E., McClelland, J. L., and Rumelhart, D. E. (1986). Parallel distributed processing: Explorations in the microstructure of cognition, vol. 1. chapter Distributed Representations, pages 77-109. MIT Press, Cambridge, MA, USA.

Hou, Y., Markert, K., and Strube, M. (2013a). Cascading collective classification for bridging anaphora recognition using a rich linguistic feature set. In Proceedings of the 2013 Conference on Empirical Methods in Natural Language Processing, pages 814-820, Seattle, Washington, USA. Association for Computational Linguistics.

Hou, Y., Markert, K., and Strube, M. (2013b). Global inference for bridging anaphora resolution. In Vanderwende, L., III, H. D., and Kirchhoff, K., editors, Human 
Language Technologies: Conference of the North American Chapter of the Association of Computational Linguistics, Proceedings, June 9-14, 2013, Westin Peachtree Plaza Hotel, Atlanta, Georgia, USA, pages 907-917. The Association for Computational Linguistics.

Inkpen, D., Paribakht, T. S., Faez, F., and Amjadian, E. (2016). Term evaluator: A tool for terminology annotation and evaluation. International Journal of Computational Linguistics and Applications, 7(2):145-165.

Ismail, A. and Manandhar, S. (2010). Bilingual lexicon extraction from comparable corpora using in-domain terms. In Proceedings of the 23rd International Conference on Computational Linguistics: Posters, COLING '10, pages 481-489, Stroudsburg, PA, USA. Association for Computational Linguistics.

Jones, M. N. and Mewhort, D. J. K. (2007). Representing word meaning and order information in a composite holographic lexicon. Psychological Review, 114:1-37.

Jordan, M. I., Ghahramani, Z., Jaakkola, T. S., and Saul, L. K. (1999). An introduction to variational methods for graphical models. Mach. Learn., 37(2):183-233.

Joulin, A., Bojanowski, P., Mikolov, T., and Grave, E. (2018). Improving supervised bilingual mapping of word embeddings. CoRR, abs/1804.07745.

Jurafsky, D. and Martin, J. H. (2009). Speech and Language Processing (2Nd Edition). Prentice-Hall, Inc., Upper Saddle River, NJ, USA.

Kageura, K. and Umino, B. (1996). Methods of automatic term recognition: A review. Terminology, 3(2):259-289. 
Kan, M., Shan, S., and Chen, X. (2015). Bi-shifting auto-encoder for unsupervised domain adaptation. 2015 IEEE International Conference on Computer Vision (ICCV), pages 3846-3854.

Kiela, D. and Clark, S. (2014). A systematic study of semantic vector space model parameters. Association for Computational Linguistics.

Kilgarriff, A., Baisa, V., Bušta, J., Jakubíček, M., Kovář, V., Michelfeit, J., Rychlý, P., and Suchomel, V. (2014). The sketch engine: ten years on. Lexicography, pages $7-36$.

Kilgarriff, A., Rychlý, P., Smrž, P., and Tugwell, D. (2004). Itri-04-08 the sketch engine. Information Technology.

Kirkpatrick, C., Alldred, B., Chilvers, C., Farahani, B., Farentino, K., Lillo, A., Macpherson, I., Rodger, J., and Trew, S. (2007). Nelson Advanced Functions. Nelson Education.

Le, Q. and Mikolov, T. (2014). Distributed representations of sentences and documents. In Jebara, T. and Xing, E. P., editors, Proceedings of the 31st International Conference on Machine Learning (ICML-14), pages 1188-1196. JMLR Workshop and Conference Proceedings.

Le Serrec, A., L'Homme, M.-C., Drouin, P., and Kraif, O. (2010). Automating the compilation of specialized dictionaries Use and analysis of term extraction and lexical alignment. Terminology, 16:77-107.

Lewis, D. (1979). Scorekeeping in a language game. Journal of Philosophical Logic, 1:339-359. 
Ljubešić, N., Vintar, Š., and Fišer, D. (2012). Multi-word term extraction from comparable corpora by combining contextual and constituent clues. In Rapp, R., Tadić, M., Sharoff, S., and Zweigenbaum, P., editors, Proceedings of 5th Workshop on Building and Using Comparable Corpora (BUCC 2012), Istanbul, Turkey.

Loginova, E., Gojun, A., Blancafort, H., Guégan, M., Gornostay, T., and Heid, U. (2012). Reference lists for the evaluation of term extraction tools. In In Proceedings of the Terminology and Knowledge Engineering Conference.

Love, S. (2000). Benchmarking the performance of two automated term-extraction systems: Logos and atao. Master's thesis, Université de Montréal.

Manning, C. D. (2015). Computational linguistics and deep learning. Computational Linguistics, 41(4):701-707.

Manning, C. D. and Schütze, H. (1999). Foundations of Statistical Natural Language Processing. MIT Press, Cambridge, MA, USA.

Marcus, M., Kim, G., Marcinkiewicz, M. A., MacIntyre, R., Bies, A., Ferguson, M., Katz, K., and Schasberger, B. (1994). The penn treebank: Annotating predicate argument structure. In Proceedings of the Workshop on Human Language Technology, HLT '94, pages 114-119, Stroudsburg, PA, USA. Association for Computational Linguistics.

Markert, K., Hou, Y., and Strube, M. (2012). Collective classification for fine-grained information status. In Proceedings of the 50th Annual Meeting of the Association for Computational Linguistics: Long Papers - Volume 1, ACL '12, pages 795-804, Stroudsburg, PA, USA. Association for Computational Linguistics. 
Markov, A. A. (1913). Essai d'une recherche statistique sur le texte du roman "Eugene Onegin" illustrant la liaison des epreuve en chain ('Example of a statistical investigation of the text of "Eugene Onegin" illustrating the dependence between samples in chain'). Izvistia Imperatorskoi Akademii Nauk (Bulletin de l'Académie Impériale des Sciences de St.-Pétersbourg), 7:153-162. English translation by Morris Halle, 1956.

Martins, A. F. T., Smith, N. A., and Xing, E. P. (2009). Concise integer linear programming formulations for dependency parsing. In Proceedings of the Joint Conference of the 4rth Annual Meeting of the ACL and the 4th International Joint Conference on Natural Language Processing of the AFNLP: Volume 1 - Volume 1, ACL '09, pages 342-350, Stroudsburg, PA, USA. Association for Computational Linguistics.

Martins, A. F. T., Smith, N. A., Xing, E. P., Aguiar, P. M. Q., and Figueiredo, M. A. T. (2010). Turbo parsers: Dependency parsing by approximate variational inference. In Proceedings of the 2010 Conference on Empirical Methods in Natural Language Processing, EMNLP 2010, 9-11 October 2010, MIT Stata Center, Massachusetts, USA, A meeting of SIGDAT, a Special Interest Group of the ACL, pages $34-44$.

Mikolov, T., Chen, K., Corrado, G., and Dean, J. (2013a). Efficient estimation of word representations in vector space. CoRR, abs/1301.3781.

Mikolov, T., Chen, K., Corrado, G., and Dean, J. (2013b). Efficient estimation of word representations in vector space. CoRR, abs/1301.3781. 
Mikolov, T., Chen, K., Corrado, G., and Dean, J. (2013c). Efficient estimation of word representations in vector space. CoRR, abs/1301.3781.

Mikolov, T., Karafiát, M., Burget, L., Cernocký, J., and Khudanpur, S. (2010). Recurrent neural network based language model. In INTERSPEECH 2010, 11th Annual Conference of the International Speech Communication Association, Makuhari, Chiba, Japan, September 26-30, 2010, pages 1045-1048.

Mikolov, T., Kombrink, S., Burget, L., Černocký, J., and Khudanpur, S. (2011). Extensions of recurrent neural network language model. In 2011 IEEE International Conference on Acoustics, Speech and Signal Processing (ICASSP), pages 55285531.

Mikolov, T., Kopecky, J., Burget, L., Glembek, O., and ?Cernocky, J. (2009). Neural network based language models for highly inflective languages. In 2009 IEEE International Conference on Acoustics, Speech and Signal Processing, pages 47254728.

Mikolov, T., Le, Q. V., and Sutskever, I. (2013d). Exploiting similarities among languages for machine translation. CoRR, abs/1309.4168.

Mikolov, T., Sutskever, I., Chen, K., Corrado, G., and Dean, J. (2013e). Distributed representations of words and phrases and their compositionality. In Proceedings of the 26th International Conference on Neural Information Processing Systems Volume 2, NIPS'13, pages 3111-3119, USA. Curran Associates Inc.

Mikolov, T., Yih, S. W.-t., and Zweig, G. (2013f). Linguistic regularities in continuous space word representations. In Proceedings of the 2013 Conference of the 
North American Chapter of the Association for Computational Linguistics: Human Language Technologies (NAACL-HLT-2013). Association for Computational Linguistics.

Mitkov, R., Evans, R., Orăsan, C., Dornescu, I., and Rios, M. (2012). Text, Speech and Dialogue: 15th International Conference, TSD 2012, Brno, Czech Republic, September 3-7, 2012. Proceedings, chapter Coreference Resolution: To What Extent Does It Help NLP Applications?, pages 16-27. Springer Berlin Heidelberg, Berlin, Heidelberg.

Mnih, A. and Hinton, G. (2007). Three new graphical models for statistical language modelling. In Proceedings of the 24th International Conference on Machine Learning, ICML '07, pages 641-648, New York, NY, USA. ACM.

Mnih, A. and Hinton, G. E. (2009). A scalable hierarchical distributed language model. In Koller, D., Schuurmans, D., Bengio, Y., and Bottou, L., editors, Advances in Neural Information Processing Systems 21, pages 1081-1088. Curran Associates, Inc.

Mnih, A. and Kavukcuoglu, K. (2013). Learning word embeddings efficiently with noise-contrastive estimation. In Burges, C. J. C., Bottou, L., Welling, M., Ghahramani, Z., and Weinberger, K. Q., editors, Advances in Neural Information Processing Systems 26, pages 2265-2273. Curran Associates, Inc.

Morin, F. and Bengio, Y. (2005). Hierarchical probabilistic neural network language model. In Cowell, R. G. and Ghahramani, Z., editors, Proceedings of the Tenth International Workshop on Artificial Intelligence and Statistics, pages 246-252. Society for Artificial Intelligence and Statistics. 
Mozer, M. C. (1995). Backpropagation. chapter A Focused Backpropagation Algorithm for Temporal Pattern Recognition, pages 137-169. L. Erlbaum Associates Inc., Hillsdale, NJ, USA.

Nazar, R. and Cabré, M. T. (2012). A machine learning approach to automatic term extraction using a rich feature set. In Proceedings of the 10th Terminology and Knowledge Engineering Conference, page 209-217, Madrid, Spain.

Nazarenko, A. and Zargayouna, H. (2009). Evaluating term extraction. In Recent Advances in Natural Language Processing, RANLP 2009, 14-16 September, 2009, Borovets, Bulgaria, pages 299-304.

Park, Y., Byrd, R. J., and Boguraev, B. K. (2002). Automatic Glossary Extraction: Beyond Terminology Identification. In Proceedings of the 19th International Conference on Computational Linguistics, pages 1-7, Morristown, NJ, USA. Association for Computational Linguistics.

Parker, R., Graff, D., Kong, J., Chen, K., and Maeda, K. (2011). English gigaword fifth edition ldc2011t07.

Pennington, J., Socher, R., and Manning, C. D. (2014). Glove: Global vectors for word representation. In Empirical Methods in Natural Language Processing (EMNLP), pages $1532-1543$.

Platt, J. (1998). Fast training of support vector machines using sequential minimal optimization. In Schoelkopf, B., Burges, C., and Smola, A., editors, Advances in Kernel Methods - Support Vector Learning. MIT Press. 
Poesio, M., im Walde, S. S., and Brew, C. (1998). Lexical clustering and definite description interpretation. In Proc. of the AAAI Spring Symposium on Learning for Discourse, pages 82-89, Stanford, CA, USA.

Poesio, M., Ishikawa, T., im Walde, S. S., and Vieira, R. (2002). Acquiring lexical knowledge for anaphora resolution. In In Proceedings of the 3rd Conference on Language Resources and Evaluation (LREC, pages 1220-1224.

Poesio, M., Mehta, R., Maroudas, A., and Hitzeman, J. (2004). Learning to resolve bridging references. In Proceedings of the 42Nd Annual Meeting on Association for Computational Linguistics, ACL '04, Stroudsburg, PA, USA. Association for Computational Linguistics.

Poesio, M. and Vieira, R. (1998). A corpus-based investigation of definite description use. Comput. Linguist., 24(2):183-216.

Poesio, M., Vieira, R., and Teufel, S. (1997). Resolving bridging references in unrestricted text. In Proceedings of a Workshop on Operational Factors in Practical, Robust Anaphora Resolution for Unrestricted Texts, ANARESOLUTION '97, pages 1-6, Stroudsburg, PA, USA. Association for Computational Linguistics.

Pontiki, M., Galanis, D., Papageorgiou, H., Manandhar, S., and Androutsopoulos, I. (2015). Semeval-2015 task 12: Aspect based sentiment analysis. In Proceedings of the 9th International Workshop on Semantic Evaluation (SemEval 2015), pages 486-495, Denver, Colorado. Association for Computational Linguistics.

Pontiki, M., Galanis, D., Pavlopoulos, J., Papageorgiou, H., Androutsopoulos, I., and Manandhar, S. (2014). Semeval-2014 task 4: Aspect based sentiment analysis. In 
Proceedings of the 8th International Workshop on Semantic Evaluation (SemEval 2014), pages 27-35, Dublin, Ireland. Association for Computational Linguistics and Dublin City University.

Prince, E. F. (1981). Toward a taxonomy of given-new information. In Cole, P., editor, Syntax and semantics: Vol. 14. Radical Pragmatics, pages 223-255. Academic Press, New York.

Rahman, A. and Ng, V. (2012). Learning the fine-grained information status of discourse entities. In Proceedings of the 13th Conference of the European Chapter of the Association for Computational Linguistics, EACL '12, pages 798-807, Stroudsburg, PA, USA. Association for Computational Linguistics.

Řehưřek, R. and Sojka, P. (2010). Software Framework for Topic Modelling with Large Corpora. In Proceedings of the LREC 2010 Workshop on New Challenges for NLP Frameworks, pages 45-50, Valletta, Malta. ELRA. http://is.muni.cz/ publication/884893/en.

Reinert, K. A. (2012). An introduction to international economics: new perspectives on the world economy. Cambridge University Press.

Roberts, C. (1998). Uniqueness in definite noun phrases. Linguistics and Philosophy, 26(3):287-350.

Rumelhart, D. E., Hinton, G. E., and Williams, R. J. (1988). Neurocomputing: Foundations of research. chapter Learning Representations by Back-propagating Errors, pages 696-699. MIT Press, Cambridge, MA, USA. 
Russell, S. J. and Norvig, P. (2003). Artificial Intelligence: A Modern Approach. Pearson Education, 2 edition.

Schmid, H. (1999). Improvements in Part-of-Speech Tagging with an Application to German, pages 13-25. Springer Netherlands, Dordrecht.

See, A., Liu, P. J., and Manning, C. D. (2017). Get to the point: Summarization with pointer-generator networks. In Proceedings of the 55th Annual Meeting of the Association for Computational Linguistics (Volume 1: Long Papers), pages 1073-1083. Association for Computational Linguistics.

Singh, R., Fedorenko, E., Mahowald, K., and Gibson, E. (2016). Accommodating presuppositions is inappropriate in implausible contexts. Cognitive Science, 40(3):607634. DOI: $10.1111 / \operatorname{cogs} .12260$.

Small, M., Kirkpatrick, C., Alldred, B., Godin, S., Lillo, A., and Dmytriw, A. (2007a). Functions 11. Nelson Education Limited.

Small, M., Kirkpatrick, C., and Dmytriw, A. (2007b). Functions and Applications 11. Nelson Education Limited.

Small, M., Kirkpatrick, C., Zimmer, D., Chilvers, C., D'Agostino, S., Duff, D., Farentino, K., Macpherson, I., Tonner, J., Williamson, J., and Yeager, T. A. (2005). Principles of Mathematics 9. Nelson Education Limited.

Smith, S. L., Turban, D. H. P., Hamblin, S., and Hammerla, N. Y. (2017). Offline bilingual word vectors, orthogonal transformations and the inverted softmax. CoRR, abs/1702.03859. 
Soon, W. M., Ng, H. T., and Lim, D. C. Y. (2001). A machine learning approach to coreference resolution of noun phrases. Comput. Linguist., 27(4):521-544.

Spenader, J. (2002). Presuppositions in spoken discourse. Doctoral dissertation, Stockholm University.

Turian, J., Ratinov, L., and Bengio, Y. (2010). Word representations: A simple and general method for semi-supervised learning. In Proceedings of the 48 th Annual Meeting of the Association for Computational Linguistics, ACL '10, pages 384394, Stroudsburg, PA, USA. Association for Computational Linguistics.

Turney, P. D. (2000). Learning algorithms for keyphrase extraction. Inf. Retr., $2(4): 303-336$.

Vasiljevs, A., Pinnis, M., and Gornostay, T. (2014). Service model for semi-automatic generation of multilingual terminology resources. In Terminology and Knowledge Engineering 2014, page 10 p, Berlin, Germany.

Versley, Y. (2007). Antecedent selection techniques for high-recall coreference resolution. In Proceedings of the 2007 Joint Conference on Empirical Methods in Natural Language Processing and Computational Natural Language Learning (EMNLPCoNLL), pages 496-505, Prague, Czech Republic. Association for Computational Linguistics.

Vieira, R. and Poesio, M. (2000). An empirically based system for processing definite descriptions. Comput. Linguist., 26(4):539-593.

Vintar, v. (2010). Bilingual term recognition revisited: The bag-of-equivalents term alignment approach and its evaluation. Terminology, 16(2):141-158. 
Vivaldi, J. and Rodríguez, H. (2007). Evaluation of terms and term extraction systems: A practical approach. Terminology, 13:225-248.

VRL, N. (2009). An unsupervised approach to domain-specific term extraction. In Australasian Language Technology Association Workshop 2009, page 94.

Vu, T., Aw, A. T., and Zhang, M. (2008). Term extraction through unithood and termhood unification. In In Proc. of Int'l Joint Conf on Natural Language Proc.

Wallach, H. M. (2006). Topic modeling: Beyond bag-of-words. In Proceedings of the 23rd International Conference on Machine Learning, ICML '06, pages 977-984, New York, NY, USA. ACM.

Wang, R., Liu, W., and McDonald, C. (2015). Corpus-independent generic keyphrase extraction using word embedding vectors. In Deep Learning for Web Search and Data Mining.

Wang, R., Liu, W., and McDonald, C. (2016). Featureless domain-specific term extraction with minimal labelled data. In Proceedings of the Australasian Language Technology Association Workshop 2016, pages 103-112.

Yang, Y., Yu, H., Meng, Y., Lu, Y., and Xia, Y. (2010). Fault-tolerant learning for term extraction. In Otoguro, R., Ishikawa, K., Umemoto, H., Yoshimoto, K., and Harada, Y., editors, PACLIC, pages 321-330. Institute for Digital Enhancement of Cognitive Development, Waseda University.

Yin, Y., Wei, F., Dong, L., Xu, K., Zhang, M., and Zhou, M. (2016). Unsupervised word and dependency path embeddings for aspect term extraction. CoRR, abs/1605.07843. 
Yoshida, M. and Nakagawa, H. (2005). Natural Language Processing - IJCNLP 2005: Second International Joint Conference, Jeju Island, Korea, October 11-13, 2005. Proceedings, chapter Automatic Term Extraction Based on Perplexity of Compound Words, pages 269-279. Springer Berlin Heidelberg, Berlin, Heidelberg.

Zervanou, K. (2010). Uvt: The uvt term extraction system in the keyphrase extraction task. In Proceedings of the 5th International Workshop on Semantic Evaluation, pages 194-197, Uppsala, Sweden. Association for Computational Linguistics.

Zhang, S., Jiang, H., Xu, M., Hou, J., and Dai, L. (2015). The fixed-size ordinallyforgetting encoding method for neural network language models. In Proceedings of the 53rd Annual Meeting of the Association for Computational Linguistics and the rth International Joint Conference on Natural Language Processing (Volume 2: Short Papers), pages 495-500. Association for Computational Linguistics.

Zhila, A., Yih, S. W.-t., Zweig, G., Meek, C., and Mikolov, T. (2013). Combining heterogeneous models for measuring relational similarity. In Proceedings of the 2013 Conference of the North American Chapter of the Association for Computational Linguistics: Human Language Technologies (NAACL-HLT-2013). Association for Computational Linguistics.

Zhong, V., Xiong, C., and Socher, R. (2018). Global-locally self-attentive encoder for dialogue state tracking. In Proceedings of the 56th Annual Meeting of the Association for Computational Linguistics (Volume 1: Long Papers), pages 1458-1467. Association for Computational Linguistics. 